\title{
Improved breast cancer screening
} for women and society:

\section{A radiological approach}

\author{
Tone Hovda
}

Institute of clinical medicine

Faculty of medicine

University of Oslo

2022

$\mathrm{UiO}:$ Faculty of Medicine University of Oslo

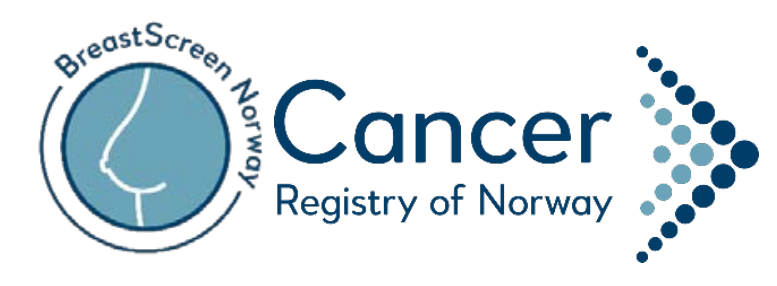


(C) Tone Hovda, 2022

Series of dissertations submitted to the Faculty of Medicine, University of Oslo

ISBN 978-82-8377-947-9

All rights reserved. No part of this publication may be reproduced or transmitted, in any form or by any means, without permission.

Cover: Hanne Baadsgaard Utigard.

Print production: Reprosentralen, University of Oslo. 


\section{Table of contents}

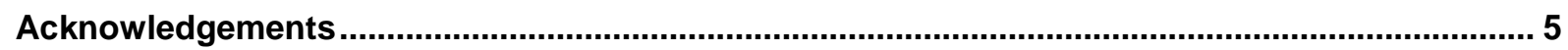

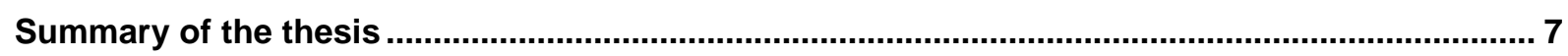

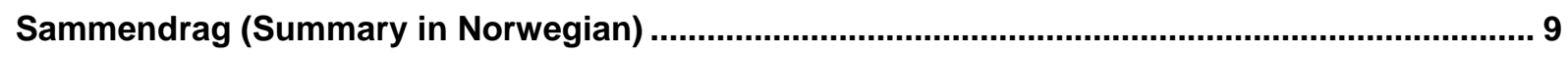

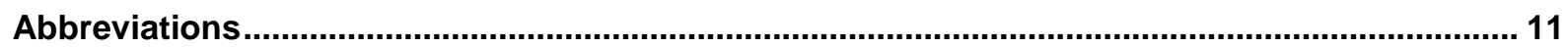

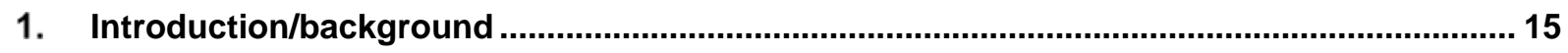

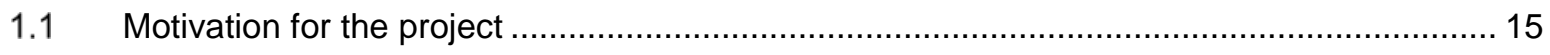

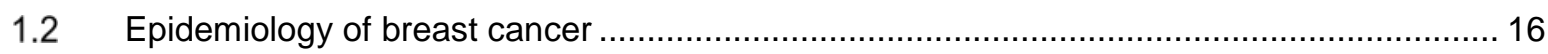

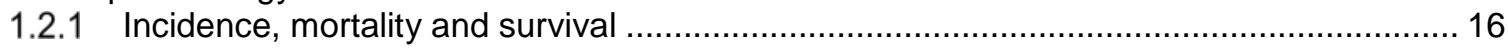

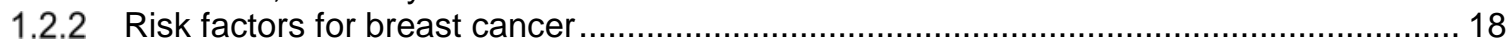

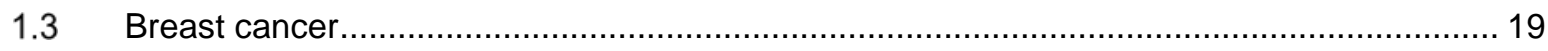

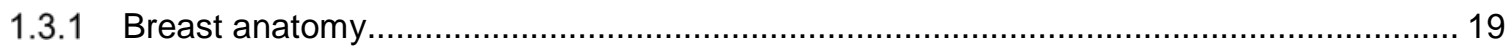

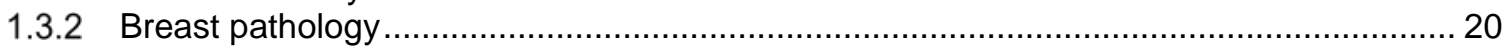

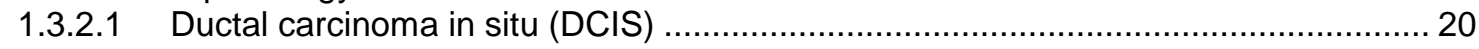

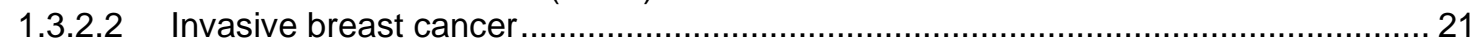

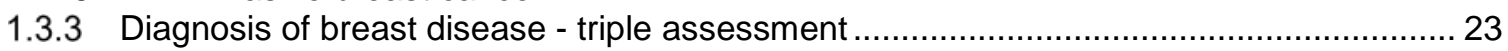

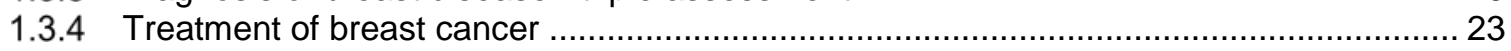

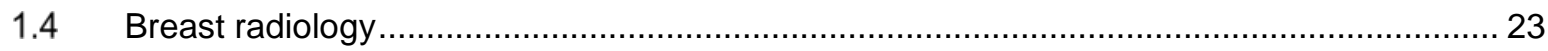

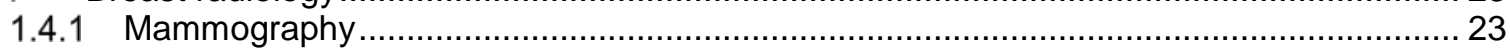

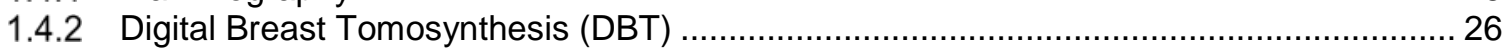

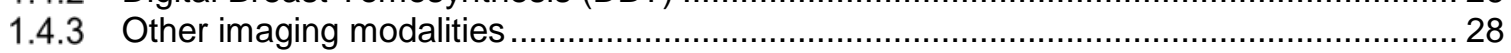

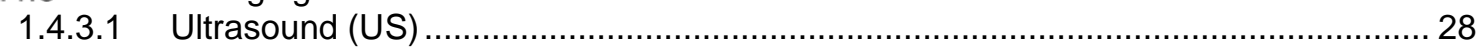

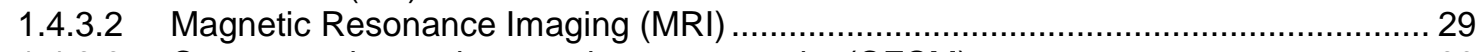

1.4.3.3 Contrast enhanced spectral mammography (CESM) ............................................ 30

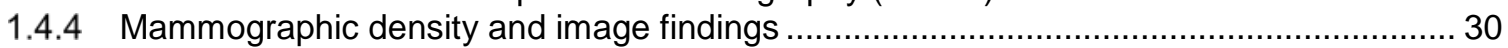

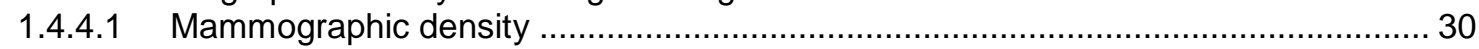

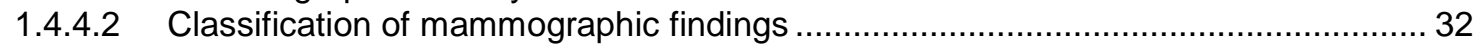

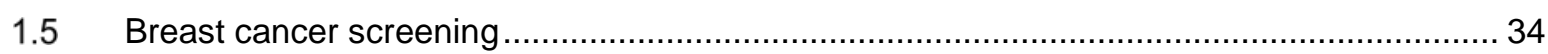

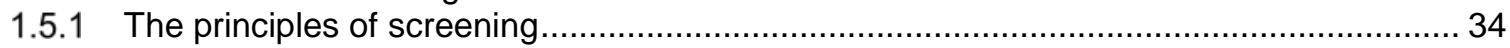

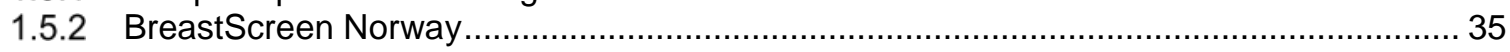

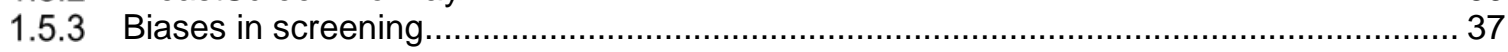

1.5.4 Effects of mammographic screening - mortality reduction, false positives and

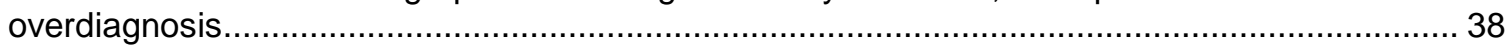

1.5.5 Challenges in mammographic screening ............................................................ 40

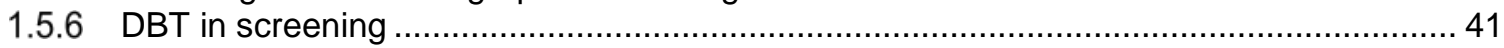

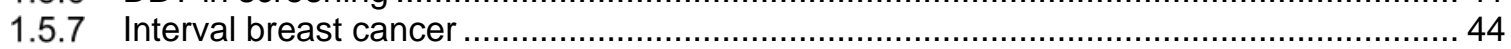

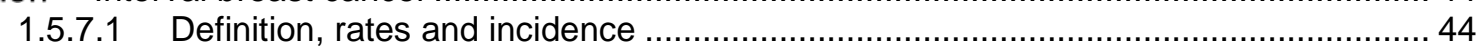

1.5.7.2 Causes and characteristics of interval cancer ..................................................... 46

1.6 Radiological review of prior screening mammograms............................................... 47

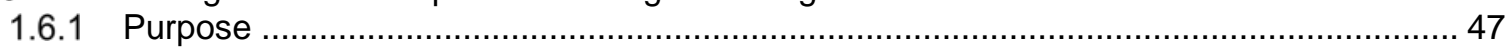

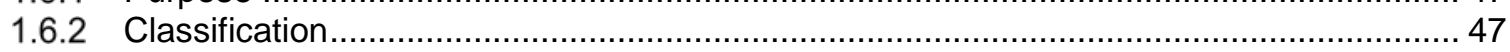

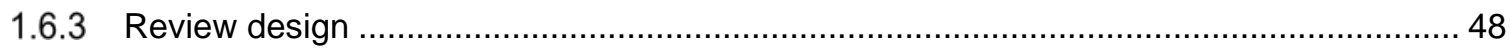

1.7 Improved breast cancer screening for women and society - a radiological approach ......... 49

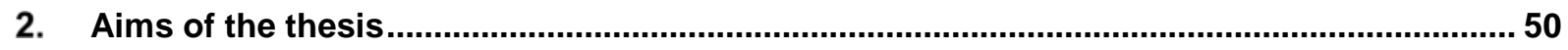

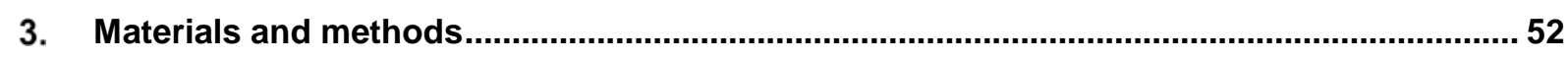

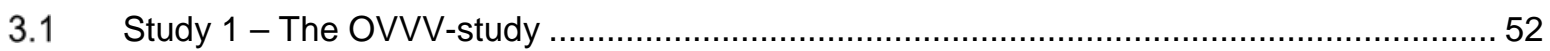

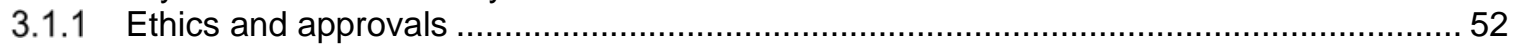




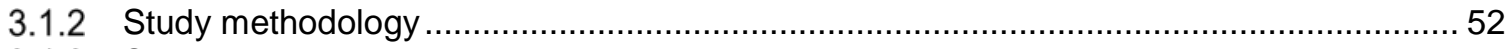

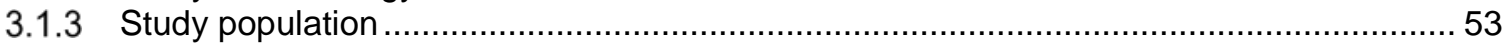

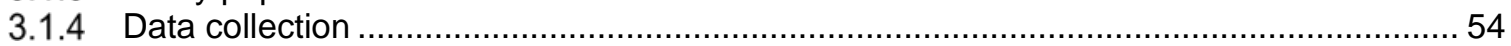

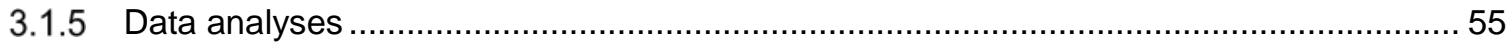

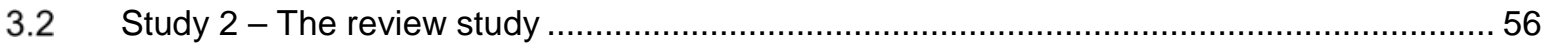

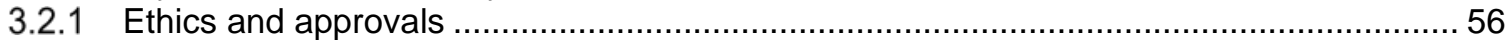

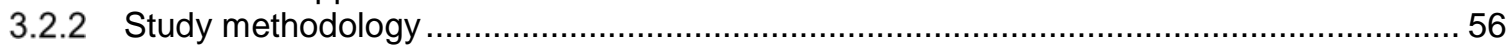

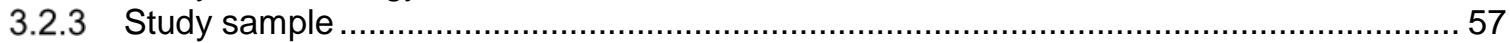

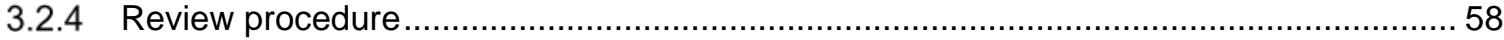

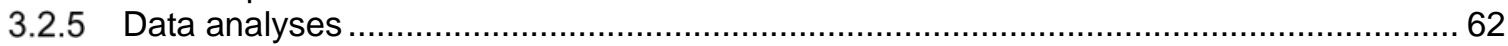

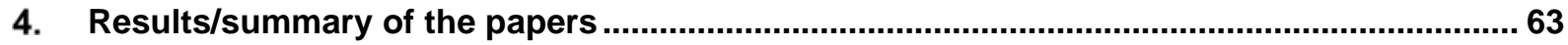

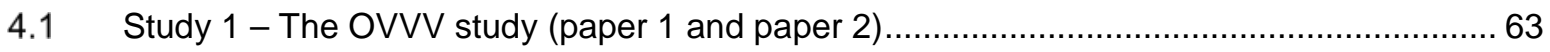

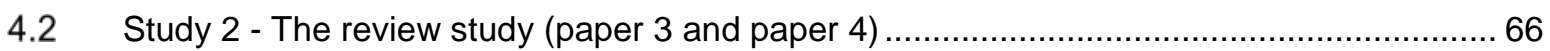

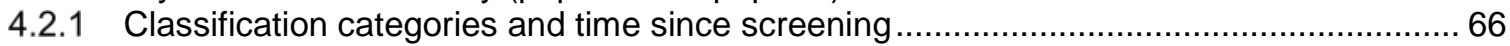

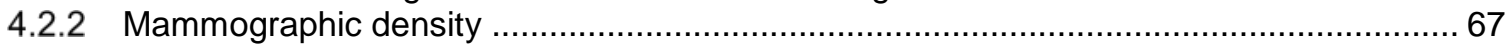

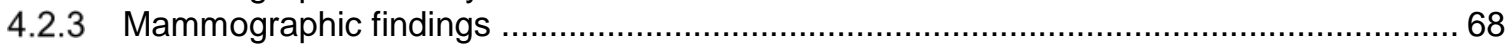

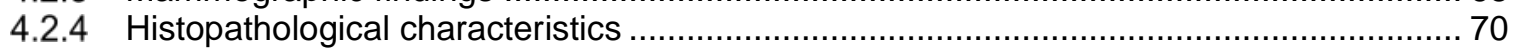

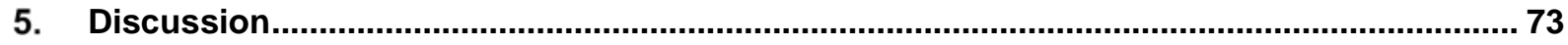

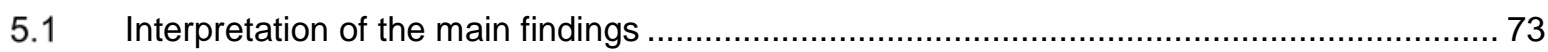

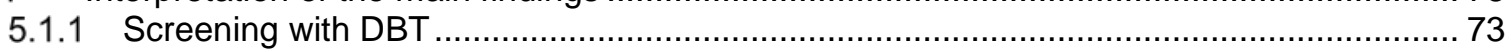

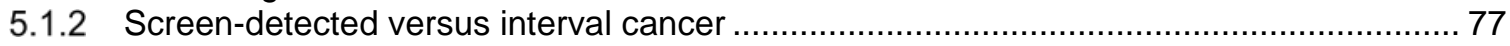

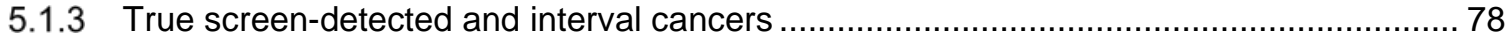

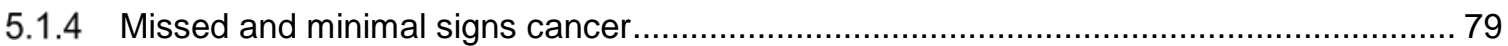

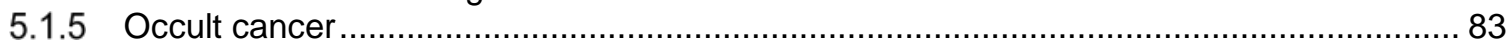

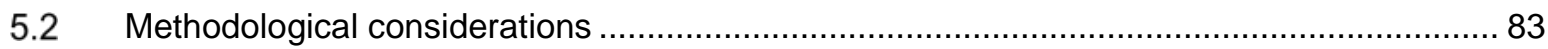

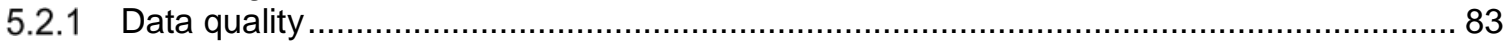

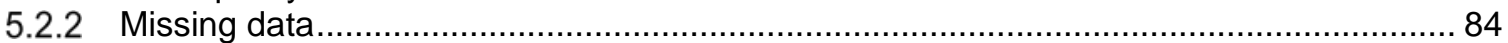

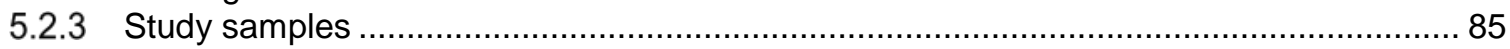

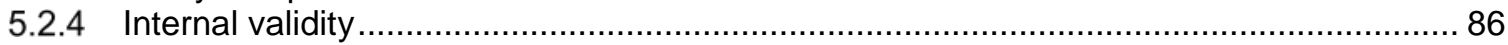

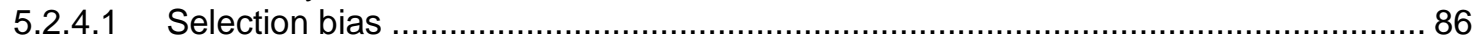

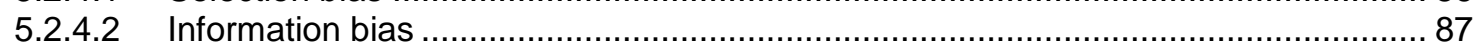

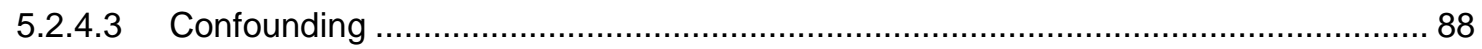

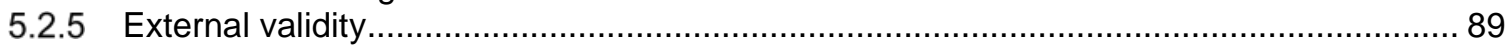

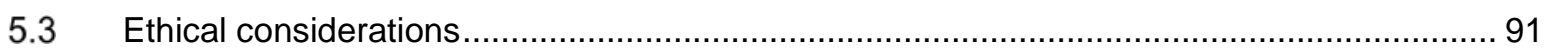

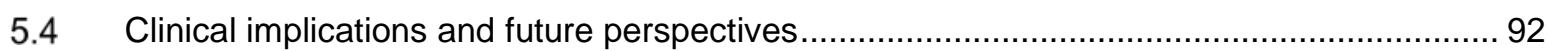

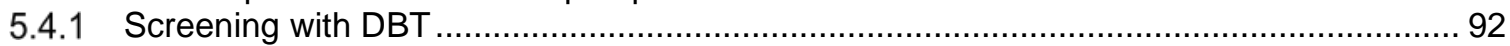

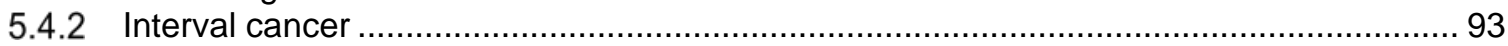

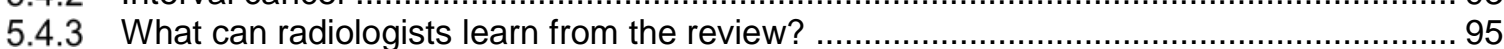

5.4.4 Artificial intelligence - future strategies to reduce the incidence of "killing cancers" ........96

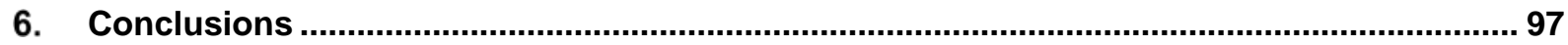

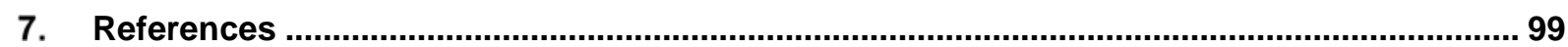

8. Papers in the thesis with supplementary material.................................................... 113 


\section{Acknowledgements}

Working on this PhD has been a journey, both personally, intellectually and professionally. I have never regretted starting this work, I am amazed by what I have learned from the process, and it is a bit surreal that the period as a PhD student will soon end.

I am forever grateful to everyone who has given me this opportunity, supported me and guided me!

To my main supervisor, Solveig Hofvind: Thank you for being so enthusiastic, always full of ideas, for sharing your almost unlimited knowledge on breast cancer screening and academic work! Thank you for being critical and constructive in all your feedback - I have learned a lot! I admire your endurance and work capacity, and you truly inspire me!

Kristine Kleivi Sahlberg, my co-supervisor: You are as enthusiastic and full of ideas as Solveig - and nothing is impossible! You are always positive, supporting, fixing everything! I am so grateful for your support and for pulling the strings! I am looking forward to future research on breast cancer in Vestre Viken! I will also thank my second co-supervisor Bjørn Naume, for being positive, supporting and inspiring!

I am extremely grateful to Vestre Viken Hospital trust for funding this $\mathrm{PhD}$, and to the Director of the Division for medical diagnostics, Wesley Caple, head of the Department of radiology, Jon-Haakon Malmer-Høvik, and head of research, development and innovation in the Division for medical diagnostics, Bjørn A. Graff for support, enthusiasm and willingness to invest in this PhD!

Linda Romundstad - my boss and head of Vestre Viken breast center - the best breast center in Norway!! Thank you for giving me the opportunity to work with breast radiology back in 2004, thanks for your teaching and guidance and for making me a - hopefully capable breast radiologist! You are a true inspiration and role model! I am also immensely grateful for you being so enthusiastic and supportive to my PhD work, which means a lot to me!

To all my radiologist colleagues at the breast center - I know there have been challenging times with shortage of staff and extra workload for all of you while I have had this opportunity to hide away from the everyday clinical work. You are all true heroes, always doing your best for our patients! Thank you for your support! 
Thank you to all my co-authors! A special thank you to Solveig Roth Hoff, for your endless but extremely valuable manuscript feedback! Kaitlyn Tsuruda - it was great to collaborate on the review study, and thank you for critically reading this thesis!

I am also extremely grateful to all radiologists who participated in the review study! I think the reviews in autumn 2016 and spring 2017 brought breast radiologists in Norway closer, we worked together for days, and had to expose our successes and mistakes to each other - for me, this was an incredible experience! Anka, Åsne and Wenche from the Cancer Registry your effort in the review study was invaluable!

Family and friends, my in-laws: thank you for support and interest in the project. To my mother: thank you for always being there, proud and supportive. Unfortunately, my father passed away during my work with this PhD project, but I know he would have been as proud and supportive as you are.

Finally, to my children Magnus, Kristin and Sigurd, you are grounding me in life, keeping me busy with other activities than work. I am immensely proud of all of you!

Eldar - you are always full of ideas, hardworking, and the best husband and father! We have been together for more than half of our lives! Thank you for supporting my work, being proud and enthusiastic! Thank you for making dinner while I am working! I love you!

Drammen, May 2021

Tone Hovda 


\section{Summary of the thesis}

Breast cancer is the most common cancer type in women worldwide and in Norway. Among the strongest risk factors for breast cancer are age, genes, mammographic density and prior breast cancer, which are factors hardly available for modification. Thus, primary prevention of the disease is challenging, and screening with mammography as secondary prevention, aimed at reducing mortality of the disease through early detection, is recommended.

BreastScreen Norway is a population-based mammographic screening program, offering biennial screening to women aged 50-69. The program started as a pilot in four counties in 1995, was gradually implemented throughout the country, being nationwide from 2006 . The screening procedure includes independent double reading by trained breast radiologists, with a consensus-based decision whether to recall the women. The same radiologists are responsible for recall assessment and diagnostics of the cancers.

The overall aim of this PhD project was to investigate ways to improve early detection of breast cancers, defined as increased detection of clinically significant, or "killing" cancers. The project included two studies, a study comparing screening with digital breast tomosynthesis (DBT) versus standard digital mammography (DM) and a retrospective, radiological review of diagnostic and prior screening mammograms from women diagnosed with screen-detected or interval breast cancer in BreastScreen Norway.

Standard screening technique in Norway is two-view DM. One limitation of the method is masking of lesions by tissue overlap and dense breast tissue. To potentially overcome this limitation, DBT was developed. DBT is a "pseudo three-dimensional" $x$-ray imaging of the breast in which multiple thin images through the breast are reconstructed, as opposed to DM. Studies have demonstrated an increased cancer detection for screening with DBT compared with DM. However, the histopathological tumor characteristics for tumors detected with DBT and the effect of DBT on interval cancer rates are not fully explored, neither is the overall impact on screening outcome for screening with DBT, including breast cancer mortality.

In the OVVV-study, women screened in Oslo 2014-2015 ( $n=34$ 641) were screened with DBT+SM, while women screened in Vestfold and Vestre Viken in the same period $(n=57$ 763) were screened with DM. We observed a 50\% increase in cancer detection for screening with DBT versus DM, in accordance with other studies. The increased detection mainly represented small, less aggressive cancers. Despite the increased cancer detection when screening with DBT, no differences in interval cancer rates were observed between the groups. Thus, we were not able to confirm that screening with DBT increased detection of 
clinically significant cancers. However, increased detection of smaller, less aggressive cancers may also decrease morbidity and mortality of the disease.

Interval cancer is breast cancer detected between two scheduled screening rounds, after a negative screening episode or after a negative recall assessment. In general, interval cancer has less favorable prognostic and predictive tumor characteristics than screen-detected cancer, and is diagnosed due to clinical symptoms. In a proportion of the interval cancers, abnormalities are retrospectively visible at the later cancer site on prior screening mammograms. These findings range from obviously missed by the screen-readers to subtle, non-specific findings. This also applies to screen-detected cancer. In the review study, panels of five radiologists reviewed diagnostic and prior screening mammograms from 1225 women diagnosed with screen-detected cancer and 1010 diagnosed with interval cancer at 16 different breast centers in Norway. Cases were classified into one of four categories according to visibility of findings at the eventual cancer site on prior screening mammograms; true, missed, minimal signs and occult screen-detected and interval cancer. We also classified mammographic findings and mammographic density and explored associations between results from the radiological review and histopathological tumor characteristics. Among the screen-detected breast cancers, $46 \%$ were classified as true, $22 \%$ as missed and $32 \%$ as minimal signs, whereas among interval cancers, $35 \%$ were classified as true, $24 \%$ as missed, $28 \%$ as minimal signs and $13 \%$ as occult. The least favorable histopathological characteristics were observed in interval versus screen-detected cancer in general and in true interval cancers in particular. Higher mammographic density was observed in interval cancer compared with screen-detected cancer, and the highest mammographic density was observed in occult interval cancer.

Thus, more than half of screen-detected and interval cancers demonstrated abnormalities at the later cancer site in an informed retrospective review of prior screening mammograms. The majority of these findings were minor and non-specific, with little or no potential for earlier diagnosis. However, in some cases obvious suspicious findings that should have prompted a recall were seen at prior screening. Increased attention and knowledge among radiologists may reduce the frequency of these cancers. This may, in turn, increase the proportion of early stage cancers, which in a longer perspective is assumed to reduce mortality from the disease. 


\section{Sammendrag (Summary in Norwegian)}

Brystkreft er det vanligste kreftformen hos kvinner, både i Norge og internasjonalt. Blant de sterkeste risikofaktorene for brystkreft finner vi alder, gener, brysttetthet og tidligere brystkreft, men alle disse faktorene er vanskelig å påvirke. Derfor er primærforebygging av brystkreft vanskelig, og screening med mammografi som sekundærforebygging er anbefalt.

Mammografiprogrammet i Norge er et populasjonsbasert screeningprogram, som tilbyr screening annet hvert år til kvinner i alderen 50-69 år. Programmet startet som en pilot i fire fylker i 1995, ble deretter gradvis innført for øvrig i landet, og var landsdekkende fra 2006. Alle mammografiundersøkelsene tydes av to uavhengige brystradiologer, og beslutningen om hvilke kvinner som skal kalles tilbake er konsensusbasert. De samme radiologene er ansvarlige for å utføre etterundersøkelser og diagnostikk av brystkreft.

Den overordnede målsetningen med dette PhD-prosjektet var å undersøke strategier for å bedre tidligdiagnostikk av brystkreft, definert som økt deteksjon av klinisk signifikant brystkreft, dvs brystkreft med et potensielt dødelig utfall. Prosjektet involverte to studier. Den ene studien sammenliknet screening med digital brysttomosyntese (DBT) og standard digital mammografi (DM), mens den andre studien var en retrospektiv regranskning av mammografibilder fra diagnosetidspunkt og foregående screening for kvinner diagnostisert med screeningoppdaget brystkreft og intervallkreft i Mammografiprogrammet.

Standard screeningteknikk i Norge er toplans DM. En begrensning ved denne metoden er at lesjoner kan maskeres av overlappende og/eller tett brystvev. For, om mulig, å redusere denne effekten, ble DBT utviklet. DBT er en «pseudo-tredimensjonal» røntgenteknikk der en rekke tynne snittbilder gjennom brystet blir rekonstruert, i motsetning til standard todimensjonal mammografi. Studier har vist en økt deteksjon av brystkreft ved screening med DBT sammenliknet med DM. Imidlertid er ikke histopatologisk svulstkarakteristikk for DBT-oppdaget brystkreft fullstendig klarlagt, og heller ikke effekten av DBT på intervallkreftraten. Den totale effekten av DBT i brystkreftscreening er heller ikke fullstendig kjent.

I OVVV-studien ble kvinner i Oslo ( $n=34$ 641) screenet med DBT mens kvinner i Vestfold og Vestre Viken ble screenet med standard DM $(n=57763)$ i den samme perioden. Vi observerte en 50\% økning i deteksjon av brystkreft ved screening med DBT sammenliknet med DM, noe som er i tråd med andre studier. Den økte deteksjonen representerte i hovedsak små, mindre aggressive svulster. Til tross for økt deteksjon av brystkreft ved 
screening med DBT, fant vi ingen forskjell i intervallkreftraten mellom de to teknikkene. Vi kunne derfor ikke bekrefte at screening med DBT øker diagnostikk av klinisk signifikante brystkreftsvulster. Imidlertid vil økt diagnostikk av små, mindre aggressive svulster også kunne redusere sykelighet og dødelighet av sykdommen.

Intervallkreft er brystkreft som diagnostiseres mellom to planlagte screeningrunder etter en negativ screeningundersøkelse, uten eller med etterundersøkelse. Intervallkreft har generelt dårligere prognose enn screeningoppdaget brystkreft, og diagnostiseres vanligvis på grunn av symptomer. Ved noen intervallkrefttilfeller kan man ved regranskning oppdage synlige forandringer på mammogrammer fra foregående screeningundersøkelse i området der kreftsvulsten ble diagnostisert. Disse forandringene varierer fra små, uspesifikke funn, til funn som er åpenbart oversett ved screeningundersøkelsen. Dette gjelder også ved screeningoppdaget kreft.

I regranskningsstudien regransket paneler bestående av 5 radiologer mammografibilder fra foregående screeningundersøkelse og diagnosetidspunkt fra 1225 kvinner med screeningoppdaget kreft og fra 1010 kvinner med intervallkreft. Tilfellene ble klassifisert som sanne, oversette, minimale tegn eller okkulte. Vi klassifiserte også mammografifunn og mammografisk tetthet, og resultatene fra den radiologiske regranskningen ble sammenholdt med histopatologiske svulstkarakteristika.

Blant de screeningoppdagede brystkrefttilfellene ble $46 \%$ klassifisert som sanne, $22 \%$ som oversett og 32\% som minimale tegn. Blant intervallkrefttilfellene ble 35\% klassifisert som sanne, $24 \%$ som oversette, $28 \%$ som minimale tegn og $13 \%$ som okkulte. Minst gunstig svulstkarakteristikk ble funnet blant intervallkrefttilfellene generelt, og spesielt blant sann intervallkreft.

Vi fant altså funn på foregående screeningundersøkelse hos omtrent en fjerdedel av brystkrefttilfellene, der tidligere diagnose kunne vært mulig. Økt bevissthet og oppmerksomhet, samt opplæring av radiologene, kan trolig redusere forekomsten av disse svulstene. Dette kan igjen øke tidlig diagnostikk av brystkreft ved screening, noe som i et lengre perspektiv antas redusere dødeligheten av sykdommen. 


\title{
Abbreviations
}

\author{
ABUS Automated breast ultrasonography \\ ADH Atypical ductal hyperplasia \\ ACR American College of Radiology \\ Al Artificial intelligence \\ BCT Breast conserving therapy \\ BI-RADS Breast Imaging Reporting and Data System \\ BRCA BReast CAncer gene \\ CC Cranio-caudal \\ CESM Contrast-enhanced spectral mammography \\ CNB Core needle biopsy \\ CT Computer Tomography \\ DBT Digital breast tomosynthesis \\ DCIS Ductal carcinoma in situ \\ DM Digital mammography \\ ECIBC European Commission Initiative on Breast Cancer \\ ER Estrogen receptor \\ FN False negative \\ FNAC Fine needle aspiration cytology \\ FP False positive \\ HER-2 Human epidermal growth factor receptor 2 \\ ILC Invasive lobular carcinoma \\ IQR Inter-quartile range \\ ITC Invasive tubular carcinoma \\ LN Lymph node \\ MBTST Malmö Breast Tomosynthesis Screening Trial \\ MLO Medio-lateral oblique \\ MRI Magnetic Resonance Imaging \\ NST Invasive carcinoma of no special type \\ OTST Oslo Tomosynthesis Screening Trial \\ OVVV Oslo-Vestfold-Vestre Viken study \\ PACS Picture archiving and communication system \\ PET Positron Emission Tomography \\ PPV Positive predictive value
}




$\begin{array}{ll}\text { PR } & \text { Progesterone receptor } \\ \text { RCT } & \text { Randomized controlled trial } \\ \text { RETomo } & \text { Reggio Emilia Tomosynthesis trial } \\ \text { SD } & \text { Standard deviation } \\ \text { SFM } & \text { Screen-film mammography } \\ \text { SM } & \text { Synthetic mammography } \\ \text { STORM } & \text { Screening with Tomosynthesis OR standard Mammography trial } \\ \text { TDLU } & \text { Terminal ducto-lobular unit } \\ \text { TN } & \text { True negative } \\ \text { TNM } & \text { Tumor, node, metastasis } \\ \text { To-Be } & \text { The Tomosynthesis in Bergen trial } \\ \text { TP } & \text { True positive } \\ \text { US } & \text { Ultrasound } \\ \text { VAB } & \text { Vacuum assisted biopsy }\end{array}$




\section{List of papers}

\section{Paper 1}

Hofvind S, Hovda T, Holen AS, Lee Cl, Albertsen J, Bjørndal H, Brandal SHB, Gullien R, Lømo J, Park D, Romundstad L, Suhrke P, Vigeland E, Skaane P. Digital Breast Tomosynthesis and Synthetic 2D Mammography versus Digital Mammography:

Evaluation in a Population-based Screening Program. Radiology. 2018; 287(3):78794.

\section{Paper 2}

Hovda T, Holen AS, Lang K, Albertsen JL, Bjørndal H, Brandal SHB, Sahlberg KK, Skaane $\mathrm{P}$, Suhrke $\mathrm{P}$, Hofvind S. Interval and Consecutive Round Breast Cancer after Digital Breast Tomosynthesis and Synthetic 2D Mammography versus Standard 2D Digital Mammography in BreastScreen Norway. Radiology. 2020; 294(2):256-64.

\section{Paper 3}

Hovda T, Tsuruda K, Hoff SR, Sahlberg KK, Hofvind S. Radiological review of prior screening mammograms of screen-detected breast cancer. Eur Radiol. 2021; 31(4):2568-79.

\section{Paper 4}

Hovda T, Hoff SR, Larsen M, Romundstad L, Sahlberg KK, Hofvind S. Missed and true interval cancer in organized mammographic screening - a retrospective review study of diagnostic and prior screening mammograms. Acad Radiol. 2021. Online ahead of print. 


\section{Introduction/background}

\subsection{Motivation for the project}

I started my residency in radiology at Buskerud Central Hospital (now Vestre Viken hospital trust) in Drammen more than twenty years ago. In 2004, I was offered a position at the breast center, and started my training as a breast radiologist. At that time, I had no experience in breast radiology, but regarded this as a motivating opportunity to specialize and gain a deeper knowledge in one particular field of the radiology universe.

The breast center in Vestre Viken is one of the largest breast centers in Norway, screening more than 20000 women every year and diagnosing more than 400 breast cancer cases annually (within the screening program and outside screening). A mainstay in our work is to provide high quality screening and diagnostics - for the benefit of patients and society.

A breast radiologist in Vestre Viken is a hybrid between a radiologist and a clinician. The radiologist is responsible for both screen-reading, diagnostic imaging and work-up at recall assessment, as well as communication of biopsy results to the patients. This means that you constantly have to face both your successes and your mistakes. Additionally, the major human aspect including patients' feedback, criticism and gratefulness is part of everyday work. Still, all this is what makes the work meaningful and motivating.

The quality of breast cancer screening is a product of the screening program's organization, logistics, screening technique and image quality as well as the performance of the radiologists in screen-reading and diagnostic work-up. For me as a radiologist, the radiologists' performance and the screening technique is of particular interest. To have the opportunity to take part in the evaluation of these aspects was the largest motivator for me to start the work with this PhD project. It was exciting to explore the effect of the novel technique digital breast tomosynthesis and synthetic mammography, and to try to understand the mechanisms behind our "successes and mistakes" in screen reading. Further, the ability to add an academic base to the more experience-based daily work as a breast radiologist has been extremely inspiring and motivating! 


\subsection{Epidemiology of breast cancer}

\subsubsection{Incidence, mortality and survival}

Breast cancer is the most common cancer type in women worldwide and in Norway. In 2020, an estimated number of 2261419 new breast cancer cases were diagnosed among females worldwide, which represented $24.5 \%$ of all new cancer cases (1). In Norway, 3726 women were diagnosed with breast cancer in 2019 , which comprised $22.9 \%$ of all cancer cases in women, and in 2018, 650 women died from the disease (2). The cumulative risk for developing breast cancer before the age of 75 is $9.0 \%$, which means that one in eleven women will be diagnosed with breast cancer before the age of 75 .

The incidence rates of breast cancer have increased over the past 50 years (Figure 1.1). The age-standardized incidence rates for women doubled from 64.3 per 100.000 person-years in the five-year period 1960-64 to 129.5 in 2015-2019 (2). The steepest increase in incidence was observed in the mid-1990s followed by a decline 2005-2009, which may be seen in the context of the introduction of population-based screening (3). BreastScreen Norway started in four counties in 1996, expanded over the next years and became nationwide in 2005 , inviting all women 50-69 years to biennial screening. The screening program is described in more detail below in section 1.5.2.

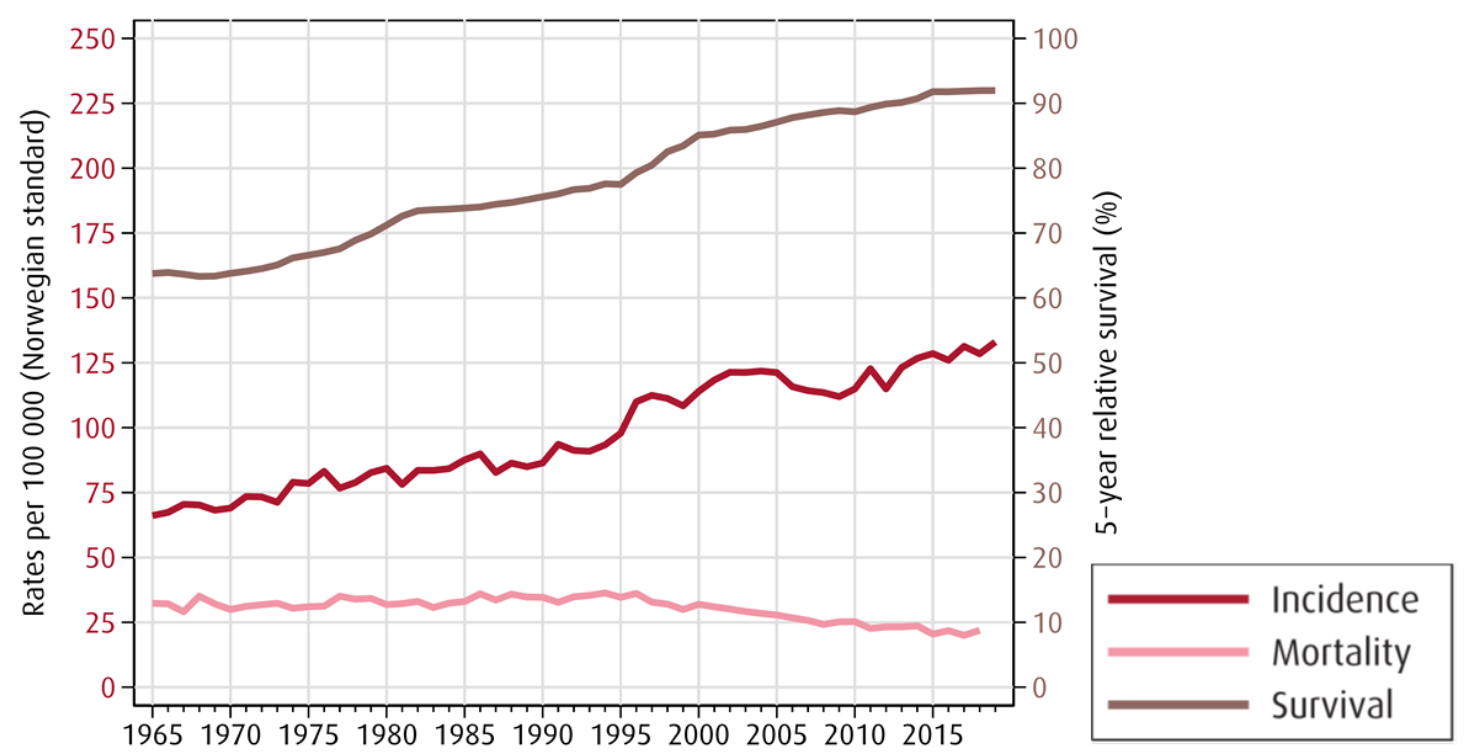

Figure 1.1: Incidence, mortality and survival of breast cancer among females in Norway 1965-2015. From: Cancer Registry of Norway. Cancer incidence, mortality, survival and prevalence in Norway, Oslo 2020 (2). 
An increase in incidence has also been observed over the last 10 years. Age-specific incidence rates indicate that this increase is mainly in the age group 70-79 years. One explanation for this may be that women reaching the upper age limit for screening (69 years) continue to have regular mammography outside the screening program (2). In addition to screening, improved diagnostics and increased breast awareness might have contributed to the increased incidence over the past decades, particularly of low stage tumors. Further, change in life-style factors and use of hormone replacement therapy might have had an effect (4).

The mortality rate for breast cancer was relatively stable until the mid-1990s, before slowly decreasing, whereas survival from breast cancer has improved steadily over the past 50 years (Figure 1.1) (2). Five-year relative survival for breast cancer $2015-2019$ was $92.0 \%$ for all, an increase from $73.7 \%$ in 1980-84, and 5-year relative survival for breast cancer by stage and period of diagnosis (1980-2019) is demonstrated in Figure 1.2. The increase in survival may be related to both earlier diagnosis by screening, improved diagnostics and increased breast awareness, as well as improved treatment $(5,6)$. For breast cancer, cumulative survival declines further with increasing time from diagnosis; 10 -year survival is $85.8 \%$ and 15 -year survival is $79.1 \%$, which indicates a persistent excess mortality. This as opposed to several other cancer forms in which the mortality rates for survivors after a certain time equal those for the general population (2).

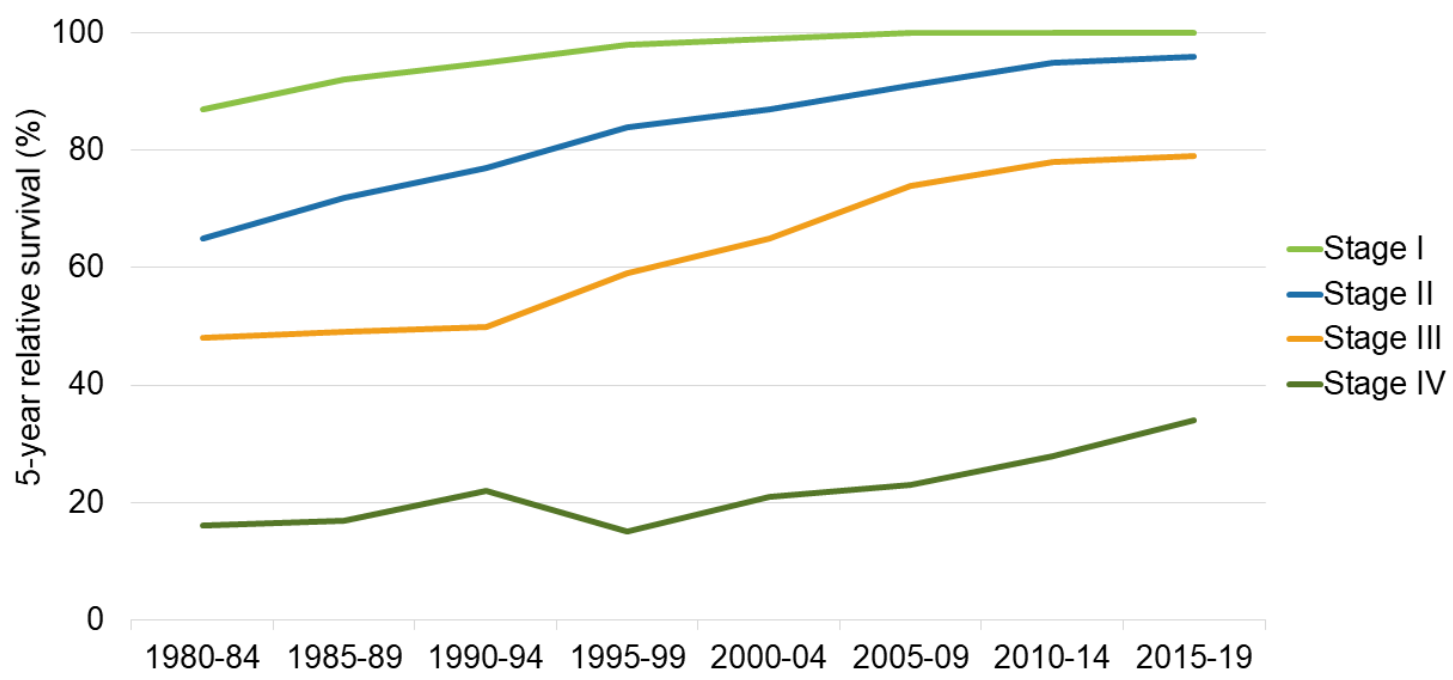

Figure 1.2: 5-year relative survival for breast cancer by stage and period of diagnosis, 1980-2019. Modified from: Cancer Registry of Norway. Cancer incidence, mortality, survival and prevalence in Norway, Oslo 2020 (2). 


\subsubsection{Risk factors for breast cancer}

Breast cancer is a multifactorial disease, and several risk factors are identified. However, for the individual women diagnosed with breast cancer, it is not possible to attribute the diagnosis to one specific risk factor as the impact of various risk factors refers to a group level, not to the individual women (4). Age is the factor strongest associated with breast cancer. With age below 50 years as reference, the relative risk for breast cancer is 9.2 for age 60-69 and 11.1 for age 70-79 (4). Only 5\% of breast cancer cases are diagnosed in women younger than 40 in Norway (2). Mammographic density is an individual factor associated with increased risk of breast cancer, a 4-6 fold higher breast cancer risk is observed in women with dense breasts (7-9). Women with a prior diagnosis of proliferative breast lesions have an increased risk for later breast cancer; usual epithelial hyperplasia is associated with a 1.0-2.5-fold increased risk, and atypical epithelial hyperplasia with a 2.54.0-fold risk (4).

Hormonal and reproductive factors like early menarche, late menopause, late age at first pregnancy and little or no breastfeeding increase the risk of breast cancer. Hormone replacement therapy is demonstrated to increase the risk of breast cancer, more in women with low body mass index, and more for combination therapy with estrogen and progestin, than estrogen only $(4,10)$. Additionally, lifestyle and exogenous factors as low physical activity levels, high body-mass index (in postmenopausal women), alcohol consumption and ionizing radiation are associated with an elevated breast cancer risk (4).

An about two-fold increased risk for developing breast cancer is observed in first-degree relatives of patients with breast cancer. Hereditary factors are estimated to account for 5$10 \%$ of all breast cancer, even more in young women. Mutations in high-penetrance genes as the BRCA 1 and BRCA 2 genes increase the breast cancer risk five-fold and account for up to $50 \%$ of the hereditary breast cancers, whereas mutations in medium and lowpenetrance genes increase the risk less and account for the rest (4).

The strongest risk factors, as age, genetic factors, mammographic density and prior history of breast cancer are hardly available for modification. Thus, primary prevention of breast cancer is challenging. 


\subsection{Breast cancer}

\subsubsection{Breast anatomy}

The main tissue components of the breast are glandular tissue, connective tissue and fat (Figure 1.3). The breast is divided into 15-25 lobes of glandular tissue, which is harboring the functional units called the terminal ducto-lobular unit (TDLU). A branching system of collecting milk ducts connect the TDLUs with the nipple-areolar complex. The lobes and ducts are lined with epithelial cells demarcated from the surrounding tissue by a basal membrane. Connective tissue and supporting ligaments named the Cooper's ligaments support and suspend the glandular tissue. The glandular and connective tissue is commonly denoted fibroglandular tissue. Variable amounts of fatty tissue is located in-between the fibroglandular tissue and subcutaneously (11).

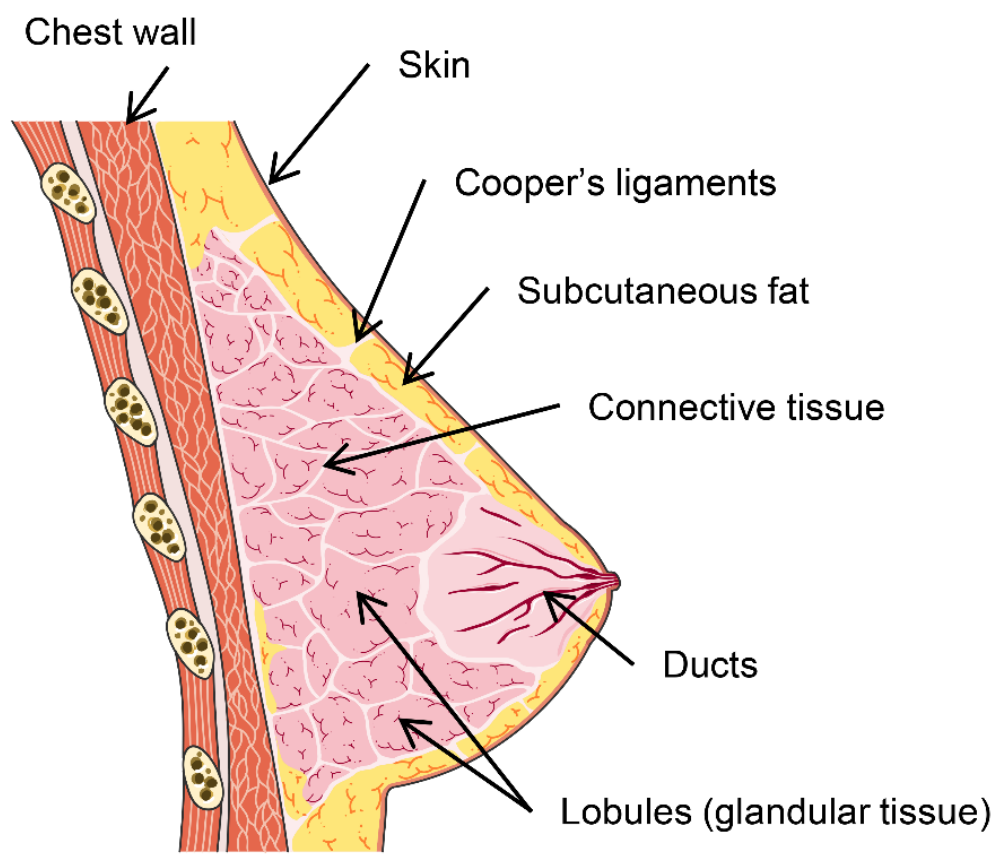

Figure 1.3. The anatomy of the breast. Modified after Servier Medical Art.

The amount of fatty tissue is associated with age, genes, hormonal status and body-mass index; at the end of the reproductive age, the glandular tissue involutes, and the amount of fatty tissue increases. The proportions of the different tissue components influence the mammographic appearance of the breast, as discussed further below. 


\subsubsection{Breast pathology}

There is a wide variety of breast lesions originating from the different tissue components of the breast, both benign and malignant. Benign lesions include among others fibrocystic changes, fibroadenomas, hamartomas, papillomas and lipomas, and will not be discussed in further detail (12). Further, proliferative breast disease, or epithelial hyperplasia is often diagnosed in breast biopsies, either associated with clinical/radiological findings or incidentally. This entity includes a wide range of pathological changes with or without atypia. Atypical ductal hyperplasia $(A D H)$ is often considered a high-risk lesion, warranting more extensive tissue sampling, as upgrading or detection of adjacent malignancies may occur (12), and may also act as a precursor for later development of invasive breast cancer (4).

Breast cancer is usually caused by an accumulation of several somatic genetic alterations that transform normal breast epithelia into malignant cells (13). Carcinomas represent the vast majority of breast malignancies. Other malignant lesions of the breast are rare and include metastases, lymphomas and sarcomas. Carcinomas originate from the epithelial lining of the lobes or ducts. If the malignant cells are restricted within the ducts, without invasion into surrounding tissue across the basal membrane, the carcinoma is defined as in situ. If the carcinoma cells invade surrounding tissue across the basal membrane, the carcinoma is invasive (12).

\subsubsection{Ductal carcinoma in situ (DCIS)}

$\mathrm{DCIS}$ is defined as malignant proliferation of epithelial cells within the ducts, with no microscopic stromal invasion of malignant cells across the basal membrane. DCIS is classified according to architectural pattern or nuclear grade. In BreastScreen Norway the Van Nuys classification is used; grade 1 is non-high grade DCIS without necrosis, grade 2 is non-high grade with necrosis, and grade 3 is defined as high grade DCIS which is composed of large pleomorphic cells with large nuclei (2.5-3x red blood cells in diameter) and frequently necrosis (12). Further, lesion size is frequently used to distinguish DCIS from $A D H$, the latter is favored for the smallest lesions (14).

DCIS is considered a non-obligate precursor of invasive cancer (15). The prognosis is excellent when treated, with 10-year survival $>95 \%$ (14). As most DCIS is treated surgically, studies on the natural course of DCIS are sparse. Observational studies on DCIS left untreated have demonstrated that up to half of the cases progress to invasive cancer (15, 16), whereas recent modeling studies demonstrated that the majority of DCIS progresses to invasive cancer $(17,18)$. However, the mechanisms associated with progression are not fully understood. 
Prior to the widespread use of mammographic screening, DCIS was usually diagnosed on the basis of symptoms, and was often of high grade at diagnosis $(12,15,16)$. Calcification of accompanying necrosis and intraductal secretions is a common feature of DCIS (19). Calcifications are usually easily visualized on mammograms, and after the introduction of mammographic screening, DCIS is most frequently diagnosed by mammographic findings rather than clinical symptoms. In the pre-screening era, DCIS usually constituted $<5 \%$ of breast cancer cases (12). This proportion has increased markedly with the introduction of screening $(15,20)$. Over the past 25 years, DCIS has contributed to $15-20 \%$ of all screendetected breast cancers within BreastScreen Norway (3).

\subsubsection{Invasive breast cancer}

In invasive carcinoma, the malignant cells invade the surrounding stromal tissue across the basal membrane, and by this, a potential for lymph node and distant metastases is established. Invasive carcinomas of the breast may be subdivided into different histological types according to morphological features (12).

Invasive carcinoma of no special type (NST) is the most frequent invasive breast cancer, constitutes up to $70-80 \%$ of all invasive cancers, and corresponds to the former entity invasive ductal carcinoma. The term includes a heterogeneous group of invasive cancers without specific characteristics to be assigned the other groups of invasive carcinoma. Thus, as the NST invasive cancer is a diagnosis of exclusion, no specific morphologic features exist, and the appearance may vary. Foci of associated DCIS are often present alongside the invasive cancer, and usually the nuclear grade of the DCIS and the invasive cancer match; low grade DCIS alongside high grade invasive cancer and vice versa is infrequently observed (12).

Invasive lobular carcinoma (ILC) is the most common special type of invasive breast cancer, and the second most common invasive cancer overall, accounting for $7-15 \%$ of the cases. ILC is in general associated with less defined lesion boundaries and a more diffuse growth pattern than NST, which may challenge the estimation of extension both clinically and radiologically. The histopathological findings of classic ILC include small to moderately sized cells that lack cohesion, thus arranged in single files or linear cords invading the stroma. This organization often results in only minor disturbance of the background tissue architecture (12).

Tubular carcinoma (ITC) usually accounts for 1-4\% of invasive breast cancers. This subtype has a favorable prognosis with a smaller tumor size, usually between 2 and $15 \mathrm{~mm}$, and a low incidence of lymph node metastasis, low incidence of recurrences and an excellent survival. They are often impossible to distinguish macroscopically from NST (12). 
Mucinous carcinoma is, like tubular carcinoma, relatively uncommon, 1-4\% of all invasive cancers. They are more common in postmenopausal women, and are often benign-looking at mammography; circumscribed and non-spiculated. They contain mucin, which is usually visible macroscopically. The prognosis of pure mucinous carcinomas is in general favorable.

Another specific type of invasive cancer is the medullary carcinoma, with a frequency like tubular and mucinous carcinomas, and in general a favorable prognosis. Other types of invasive cancer include among others papillary carcinoma, adenoid cystic carcinoma and metaplastic carcinoma. Additionally, mixed carcinomas of all the above types, including the NST occurs.

Invasive breast cancer is classified into histological grade 1-3 based on the extent of tubule formation, nuclear pleomorphism and mitotic counts (12). Other important prognostic factors include tumor diameter, lymph node status and $\mathrm{Ki}-67$, which is a cellular marker for proliferation. Estrogen receptor (ER), progesterone receptor (PR) and human epidermal growth factor 2 (Her2) are the standard biomarkers in breast cancer.

From the early 2000s, breast cancer has been divided into different molecular subtypes separated by gene expression profiles, with various biological properties and prognosis, and over the past years, commercial tests for genetic signature have become commercially available (21). However, still, subtypes classified by immunohistochemical markers are commonly used as proxies for the molecular subtypes. Most widely used is the St. Gallen classification system (22) (table 1.1).

Table 1.1. Molecular subtypes, according to St Gallen 2013. ER: estrogen receptor; PR: progesterone receptor, Her2: Human epidermal growth factor 2. The threshold for "low" and "high" Ki-67 may vary between laboratories, and should be indicated separately in studies.

\begin{tabular}{ll} 
Subtype & Characteristics \\
\hline Luminal A-like & ER+, PR+, Her2-, Ki-67 «low» \\
Luminal B-like Her2 negative & ER+, Her2- and Ki-67 «high» and/or PR- \\
Luminal B-like Her2 positive & ER+, Her2+, any Ki-67/PR \\
Her2 positive & Her2+, ER-, PR-, any Ki-67 \\
Triple negative & ER-, PR-, Her2-, any Ki-67 \\
\hline
\end{tabular}

Invasive cancer is clinically (pre-surgical) and pathologically (post-surgical) staged according to the TNM-system, grouping patients into one of four stages based on tumor size $(T)$, regional lymph node status $(\mathrm{N})$ and presence/absence of distant metastases (M) (12). 


\subsubsection{Diagnosis of breast disease - triple assessment}

Modern diagnosis of breast disease in general and breast cancer in particular is performed multidisciplinary by trained professionals. The gold standard for diagnosis is the triple assessment, a triad of clinical examination, radiological imaging and pathological sampling (23). Clinical breast examination may be performed by the imaging radiologist and/or clinician. The main imaging methods are mammography and ultrasound, which are discussed in further detail in chapter 1.4. Tissue sampling may include core needle biopsy (CNB), vacuum assisted biopsy (VAB) or fine-needle aspiration cytology (FNAC, mainly for cysts or lymph nodes). Tissue sampling is usually made under ultrasound or x-ray guidance. In case of negative imaging findings and suspicious findings at clinical examination, sampling may be guided by the clinical findings (23-25).

\subsubsection{Treatment of breast cancer}

Treatment of breast cancer is multimodal, and includes surgery, radiation therapy, systemic treatment with chemotherapy, and/or hormonal treatment and targeted therapy. The vast majority of breast cancer patients undergo surgery, either breast conserving therapy (BCT) or mastectomy. In Norway, $81 \%$ of women with breast cancer with tumor diameter $<30 \mathrm{~mm}$ underwent BCT in 2019 (26). Radiotherapy is indicated to prevent loco-regional recurrence after BCT and in lymph node positive disease (26). Neo-adjuvant systemic therapy prior to surgery has been offered patients with locally advanced disease for many years, and is over the past years also offered women with large, but not locally advanced, and/or more aggressive tumors. In 2019, 16\% of patients diagnosed with breast cancer received neoadjuvant therapy (26). The need for and choice of systemic treatment are based on stage at diagnosis and molecular features of the disease $(4,27)$.

\subsection{Breast radiology}

\subsubsection{Mammography}

Mammography is the primary imaging technique of the breast. The first radiologic imaging of the breast was performed in 1913, and different techniques were used during the next 50 years. From the late 60s/early 70 s, dedicated mammographic systems and screen-film mammography (SFM) was developed and utilized. During the last two decades a transition from SFM to full-field digital mammography (DM) has occurred, and DM has fully replaced SFM in Norway (Figure 1.4) (28). 


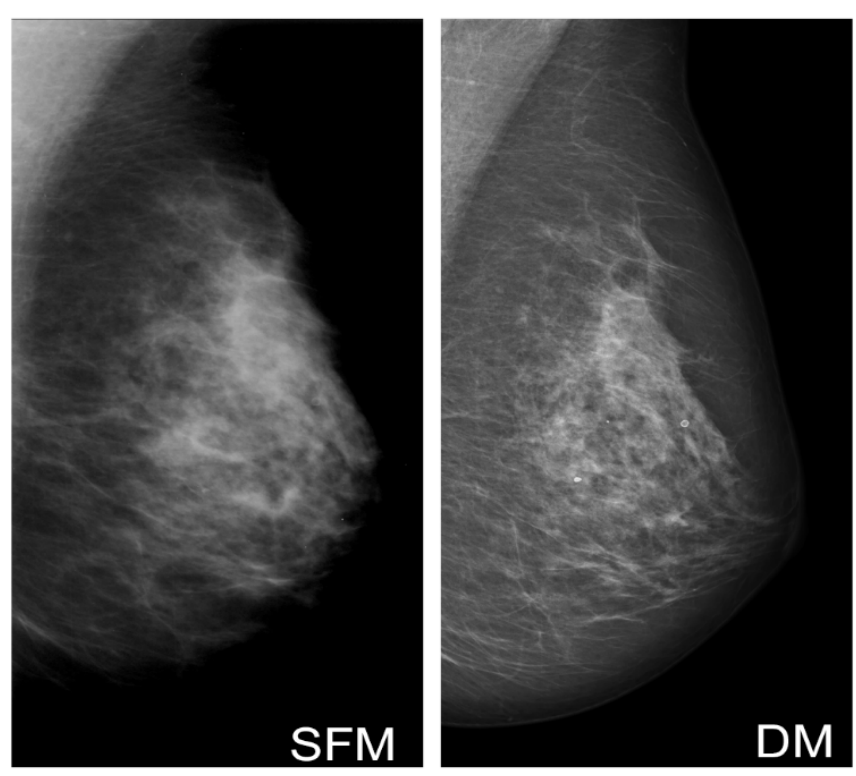

Figure 1.4: Screen-film mammography (SFM - left) and full-field digital mammography (DM - right) of the left breast from the same woman.

Advantages of DM to SFM include a wider dynamic range, a better contrast resolution, especially in dense breast tissue, as well as post-processing opportunities. The dose is slightly lower in DM than SFM. DM also has advantages to SFM regarding workflow, reduction in recall for technical reasons, image storage and subsequently development of new techniques as digital breast tomosynthesis (described in detail below) and contrastenhanced mammography as well as facilitating computer-aided detection (29-31).

Dedicated mammographic systems enable optimal image contrast and resolution, and also facilitate positioning of the breasts to include as much information as possible in the images. The mammography unit includes an anode $x$-ray tube with matched filtration for soft tissue imaging. The most common anode/filter combination is molybdenum/molybdenum. Further, the mammography unit consists of a moving grid to reduce scattered radiation, an x-ray image detector and an automated exposure control device (Figure 1.5). During the image acquisition, the breast is compressed between a compression paddle and the detector. Compression of the breast is important to stabilize the breast and reduce/avoid image blurring from breast movement, and to spread the normal fibroglandular tissue to better reveal pathological findings. Further, the compression leads to a reduced and more uniform breast thickness, and thereby reduces the radiation dose and exposure time (32).

A standard mammography exam consists of two views of each breast (Figure 1.6). In the medio-lateral oblique (MLO) projection, the breast is compressed obliquely, with the pectoral muscle included in the field of view, and with the direction of the x-ray beam perpendicular to the detector. In the cranio-caudal (CC) projection, the breast is compressed in the horizontal 
plane and the x-ray beam is vertically directed. Supplementary views, including magnification or spot compression views, may be added to the exam when appropriate for diagnosis, in particular to characterize calcifications more thoroughly.

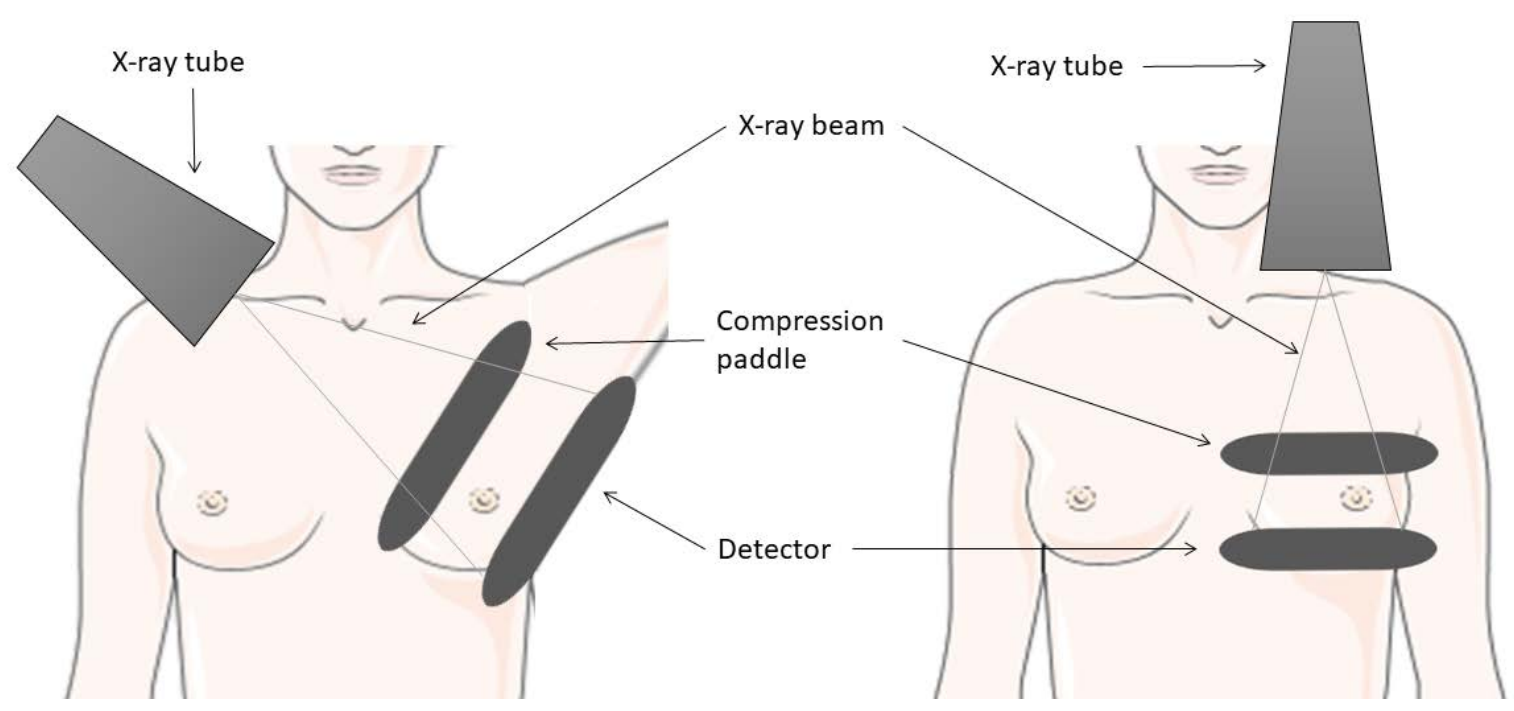

Figure 1.5: Mammography. The breast is placed between a compression paddle and the detector. Left: Medio-lateral oblique (MLO) projection with oblique direction of the $x$-ray beam from the $x$-ray tube towards the detector. Right: Craniocaudal (CC) projection with vertical direction of the $x$-ray beam. (Modified from Servier Medical Art).
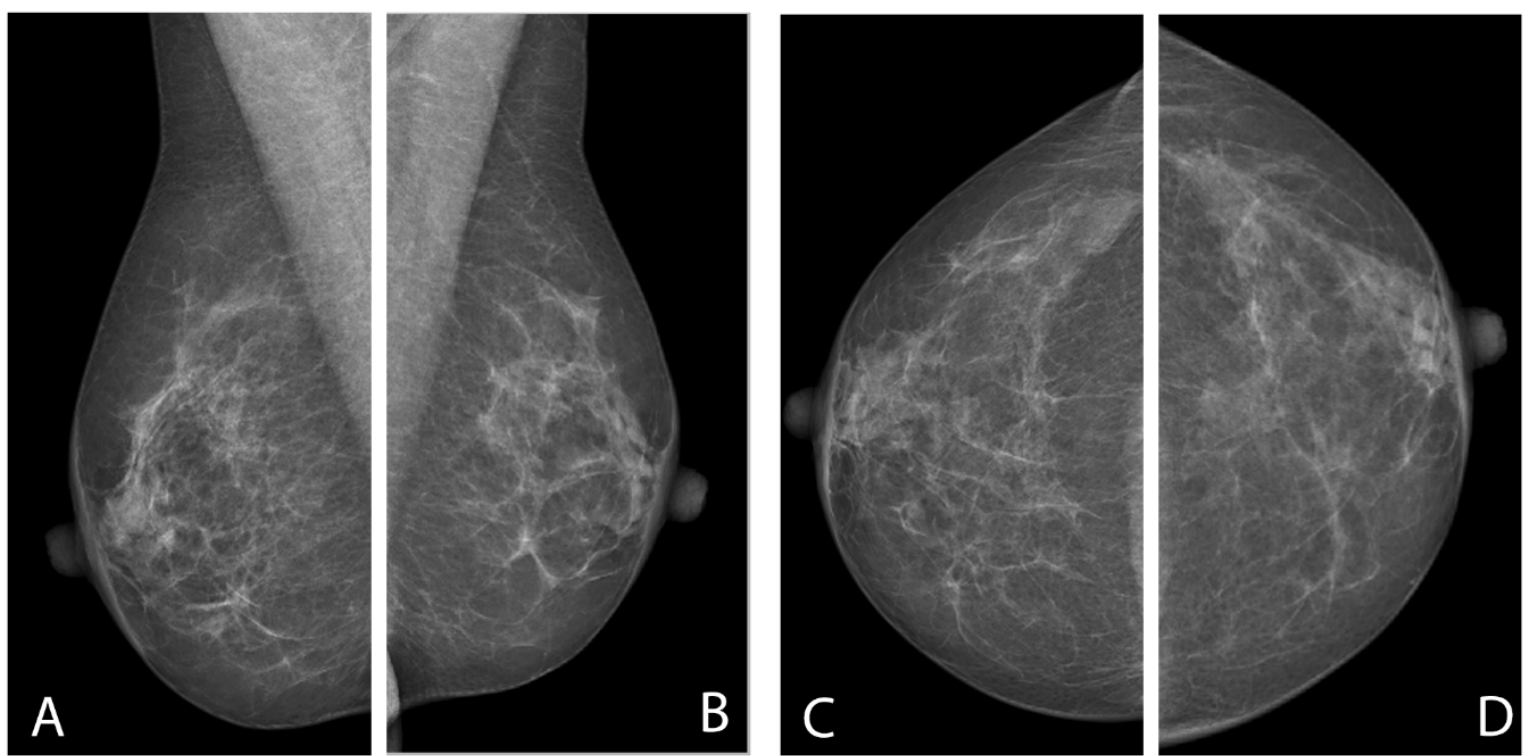

Figure 1.6: Standard projections in mammography. Right $(A)$ and left (B) medio-lateral oblique (MLO) projection; right (C) and left (D) craniocaudal projection.

Mammographic imaging of the breast demands high spatial resolution, as many of the objects of interest in the images are very small with tiny details and fine borders. Further, excellent soft-tissue contrast is crucial, as the range of tissue densities is limited in the 
breast, in contrast to e.g. chest x-ray or skeletal imaging. Third, in mammographic screening which involves regular imaging and a substantial number of repeated mammograms in asymptomatic women, it is important to keep the radiation doses as low as possible without compromising the image quality (29). Adequate and standardized positioning is crucial to obtain the necessary information from images and optimal comparison of consecutive examinations. Optimization of image quality is important in mammography, both in clinical and screening setting. Image quality is affected by factors related to acquisition and postprocessing as well as positioning (33).

\subsubsection{Digital Breast Tomosynthesis (DBT)}

One limitation of DM as a two-dimensional imaging technique, is reduced sensitivity due to overlap of tissue structures. This includes both normal/dense breast tissue overlapping breast lesions, as well as formation of "pseudo-lesions" due to superimposition of different layers. DBT was developed to address the limitations of mammography related to masking and overlap of tissue structures. In DBT, an arcuate movement of the x-ray beam over the compressed breast acquires multiple low-dose projection views. A reconstruction algorithm processes these projection views into several thin image plans parallel to the breast support plates (34-36). The technique enhances the information present in the current plane, while blurring information in the other parts of the breast. This might facilitate an improved lesion demarcation and a reduction of the masking effect of overlapping tissue (37). The DBT images are acquired in the same projections as DM images, and DM and DBT images may be acquired in the same compression (Figure 1.7).

The radiation dose acquired with DBT is demonstrated to be equal to or slightly higher than standard DM $(38,39)$. Further, it is essential to obtain a two-dimensional (2D) image in addition to DBT, to facilitate comparison with prior images and for optimal presentation of calcifications. The combination of a DM and a DBT acquisition thus results in an almost doubled radiation dose, which is controversial in screening. To overcome this problem, software algorithms were developed, which reconstruct a synthetic 2D image (synthetic mammogram, SM) from the "three-dimensional" (3D) DBT exam. Hence, both a 2D and a 3D presentation are obtained from the same exposure and compression, and the radiation dose of the exam is comparable to a standard DM acquisition (Figure 1.8). This reduces the overall radiation dose (40-42). Several studies have shown that synthetic mammograms in combination with DBT are non-inferior to DM+DBT in screening including cancer detection and accuracy $(41,43-47)$. 


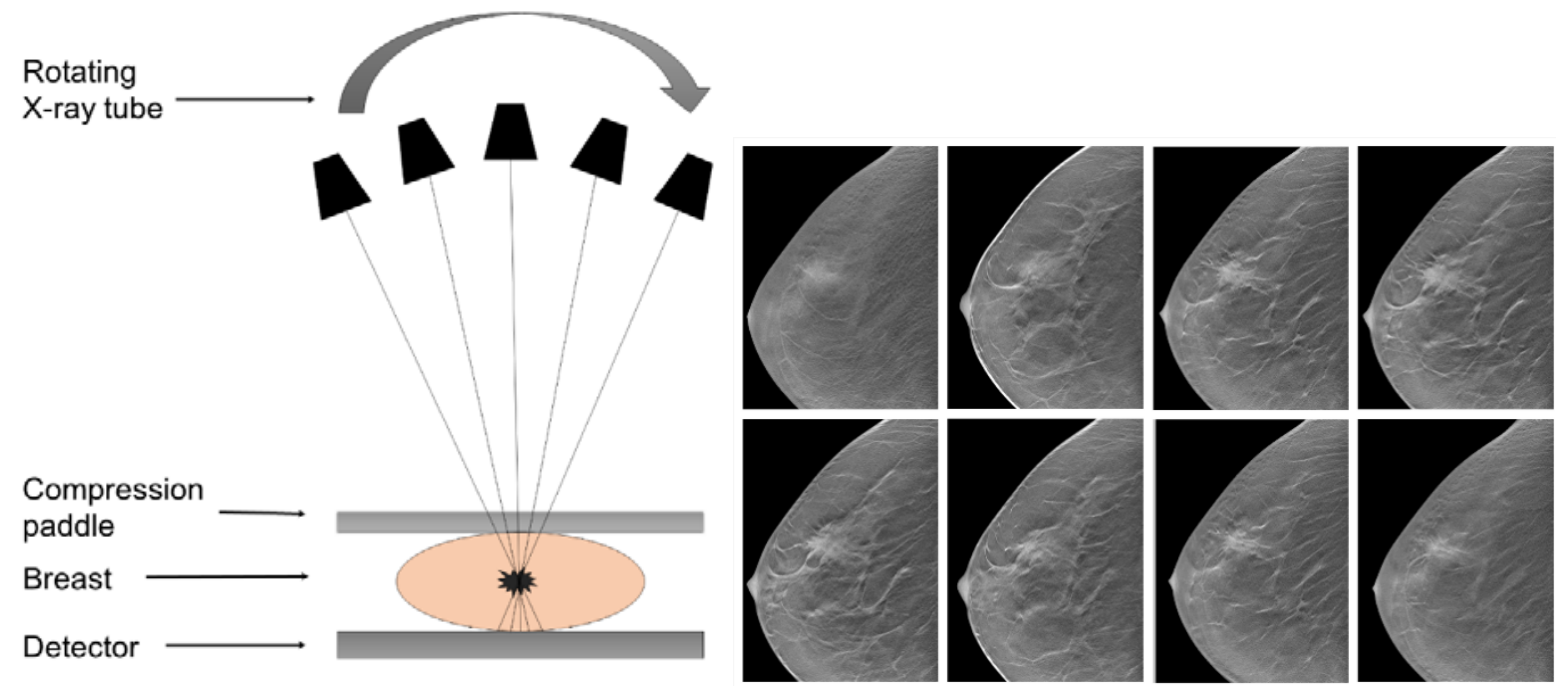

Figure 1.7: Acquisition of digital breast tomosynthesis images: The $x$-ray tube moving in an arcuate direction acquires multiple low-dose projection views of the breast (left), and a reconstruction algorithm processes these projections into several thin images of the breast (right).
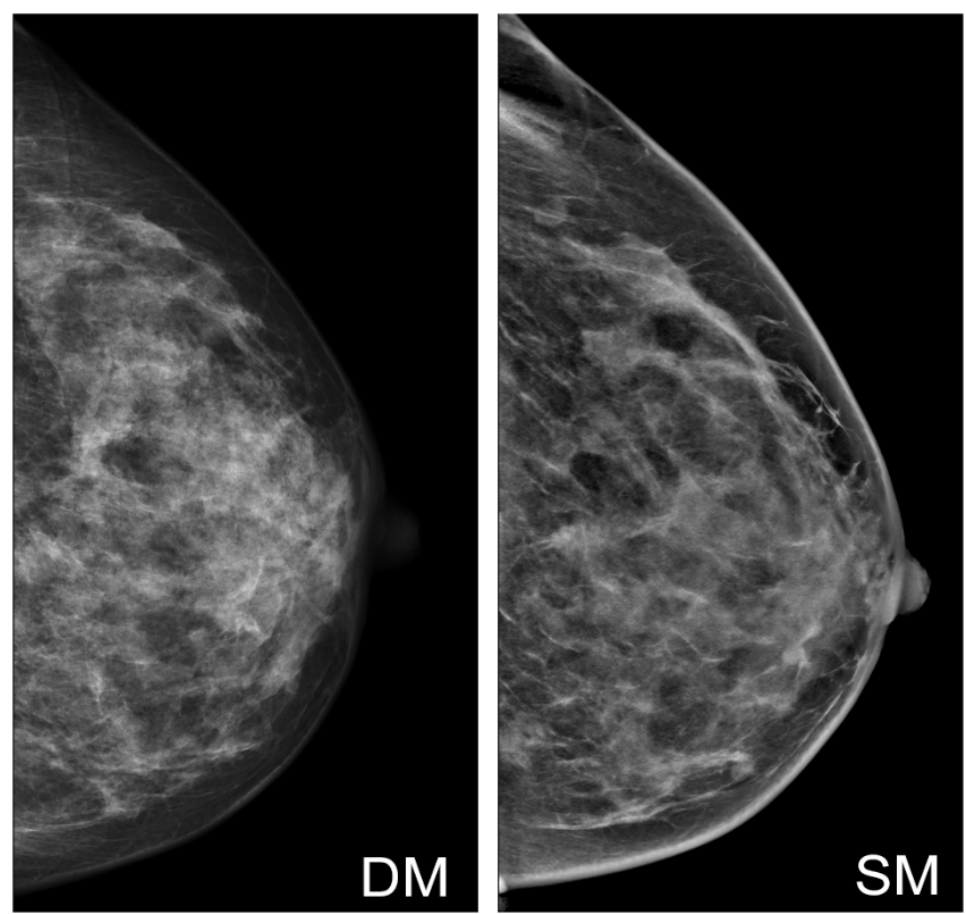

Figure 1.8. Digital mammogram - DM (left) and synthetic mammogram - SM (right) of the same breast in the craniocaudal projection.

DBT was first introduced to the peer-reviewed literature in 1997 by Niklason (48) and after a gap of 10 years, several small and moderate-sized observational and reader studies were performed with mammograms obtained from both screening and a clinical setting, demonstrating non-inferiority of DBT to DM regarding sensitivity (49-53). However, in these early reader studies, most of the study samples were cancer-enriched, and most cancers 
were originally diagnosed with DM, causing a possible bias for DBT. A large multiinstitutional multi-reader study conducted in the United Kingdom in 2011 observed no difference in sensitivity between DM and DM+DBT, while the specificity was higher for DM+DBT compared with DM. However, the study sample was cancer enriched and biased as the majority of the women were recruited as recalls from screening due to abnormalities detected with DM $(54,55)$.

Over the next years, large scale, retrospective and prospective screening studies were conducted, comparing screening with DBT in combination with DM or SM to screening with standard DM (39, 45, 56-65). In short, most studies demonstrated a higher cancer detection rate for screening with $\mathrm{DBT}+\mathrm{DM} / \mathrm{SM}$ compared with $\mathrm{DM}$, with no decline in interval cancer rates, whereas the recall rates varied. The studies will be discussed in more detail in chapter 1.5.6. The effect of screening with DBT on breast cancer mortality is not yet explored due to the requirement of at least ten years of follow-up of the women, after diagnosis.

\subsubsection{Other imaging modalities}

\subsubsection{Ultrasound (US)}

US is based on the transmission of ultrasound from a transducer through breast tissue in a perpendicular direction and back to the transducer (echo). The differences in acoustic properties of the various tissue types and abnormalities of the breast result in different strength of the echoes. Consequently, the tissue structures are displayed in various shades of gray based on the intensity of the returning echoes (Figure 1.9) (66).
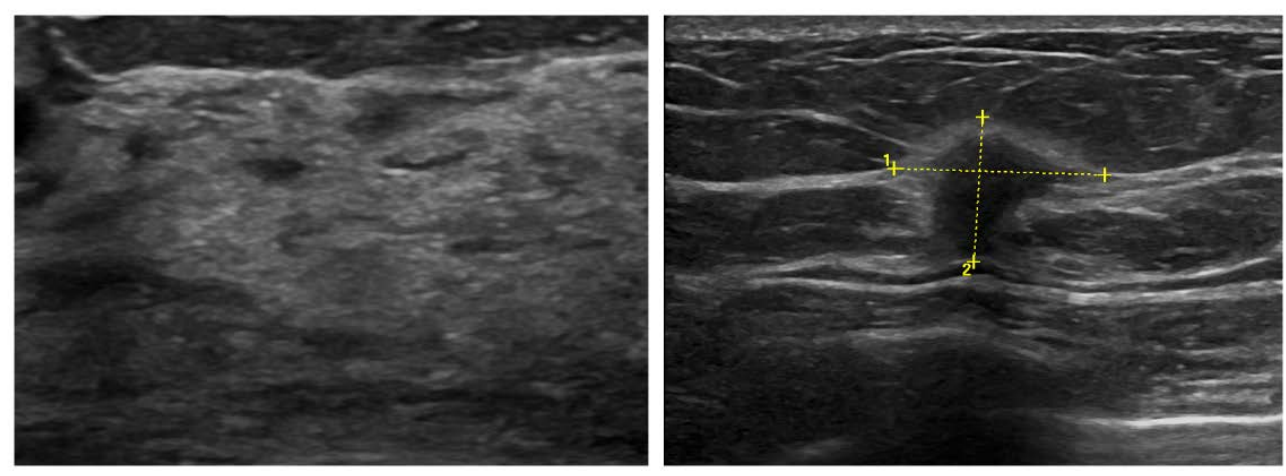

Figure 1.9: Ultrasound images of normal fibroglandular tissue (left) and a malignant tumor (right).

US is an important supplement to mammography for women recalled due to mammographic findings at screening and for symptomatic women, both by adding diagnostic information as well as being a tool for interventional procedures. Further, both hand-held and automated breast ultrasound (ABUS) has the potential to increase cancer detection as a supplementary 
screening tool to standard mammography in women with dense breasts (67-69). However, a major increase in suspicious findings resulting in recall and false positive screening results has been demonstrated, which balances out the positive effect on increased detection (68, $70,71)$.

\subsubsection{Magnetic Resonance Imaging (MRI)}

Contrast enhanced breast MRI is the most sensitive imaging method for breast cancer detection, both in symptomatic and asymptomatic women, with a higher sensitivity than mammography, DBT and US (72). The pooled sensitivity of contrast enhanced MRI is estimated $91 \%$, with a pooled specificity of $73 \%(72,73)$. As a malignant tumor grows, the metabolic needs increase, resulting in a release of peptides that promote angiogenesis and changes in the tumor's microvascularity. As a result, the vascular permeability increases, which is usually associated with a rapid uptake and wash out of the administered contrast medium. This contrast kinetics results in variations in signal intensity characteristic for malignant tumors (Figure 1.10). Additionally, morphological features are also important in image interpretation of breast MRI (74). Established indications for breast MRI are staging of breast cancer, screening of high-risk women (in particular gene-mutation carriers), and response evaluation of neo-adjuvant therapy. Population-based screening of women at average risk is debated, mainly due to the limitations associated with costs, time consumption and availability, and also increased false-positive exams. However, abbreviated protocols with markedly reduced acquisition and interpretation time have been developed, and a multicenter trial from the Netherlands has demonstrated an increased cancer detection rate (16.5/1000) and a reduced interval cancer rate in women with extremely dense breasts screened with supplementary abbreviated MRI $(72,73,75-79)$.

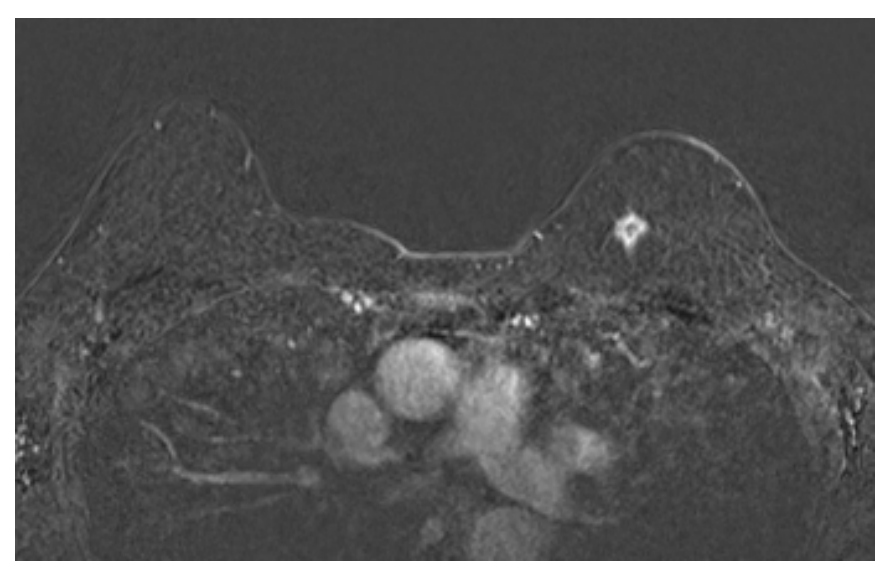

Figure 1.10: MRI of an irregular, contrast-enhancing malignant tumor in the left breast. 


\subsubsection{Contrast enhanced spectral mammography (CESM)}

CESM is a technique utilizing dual energy subtraction mammography and an intravenous iodine contrast agent. Low energy and high energy image acquisition occur during a single compression in each of the standard views after injection of an intravenous contrast agent. The low energy image corresponds to a standard DM image, and is evaluated along with a recombined image resulting from subtraction of the low energy image from the high energy image (80). The mechanisms for contrast enhancement in CESM are similar to MRI, primarily based on the altered circulatory conditions and neo-angiogenesis. Indications for CESM include evaluation of extent of disease, response to neo-adjuvant therapy as well as diagnostic work-up in screening patients. Sensitivity is demonstrated to be similar to or slightly lower than MRI, though significantly higher than standard DM, while specificity is in studies higher than for MRI $(80,81)$. Benefits of CESM to MRI may include availability, reduced equipment costs and use in patients with contraindications for MRI. Limitations of CESM to MRI include artefacts and limited evaluation of axillary regions and breast implant impairment (80).

\subsubsection{Mammographic density and image findings}

\subsubsection{Mammographic density}

Mammographic density refers to the mammographic appearance of breast tissue composition and the amount of fibroglandular tissue relative to fatty tissue (82). High mammographic density may be associated with a masking effect on tumors and decreased sensitivity of mammography, which may impair early diagnosis of malignancy $(7,9,82)$. Mammographic density is influenced by age, genes, hormonal status including use of hormonal replacement treatment, and body-mass index (9), and is an independent risk factor for breast cancer. The association between mammographic density and breast cancer risk is stronger than most other known risk factors, except age and high-risk genetic factors. A 4-6 fold increased risk for breast cancer has been demonstrated in women with the highest versus lowest density categories (9). The distribution of mammographic density in a population is highly dependent on the population characteristics, in particular age. Further, different classification systems for mammographic density exist.

Wolfe was the first to classify mammographic density or breast parenchymal pattern into a visual and qualitative system, based on the proportions and distribution of fat, epithelial tissue, connective tissue, and the so-called "prominent ducts". The different categories were associated with different risks for developing breast cancer (83). The Tabar classification is based on an anatomic-mammographic correlation (84), describing the parenchymal pattern in five different categories, based both on the amount of fatty and more dense breast tissue, 
as well as the appearance of the fibroglandular tissue. Additionally, quantitative classification methods have been introduced, either visually or computer-aided, in which the breasts are assessed and classified according to percentage of dense breast tissue or absolute mammographic density $(7,9,85)$.

The most widespread classification system for mammographic density today is the American College of Radiology (ACR) Breast Imaging Reporting and Data System (BI-RADS $®$ ) classification system (86). According to BI-RADS, mammographic density is classified into either of four categories. The $4^{\text {th }}$ edition was based on percentage density, but was replaced by the $5^{\text {th }}$ edition in 2013 with a more qualitative approach, omitting the percentages (87) (Table 1.2, Figure 1.11).

Table 1.2. BI-RADS $5^{\text {th }}$ edition mammographic density categories

\begin{tabular}{ll} 
Category & Description \\
\hline BI-RADS a & Almost entirely fatty \\
BI-RADS b & Scattered areas of fibroglandular density \\
BI-RADS c & Heterogeneously dense, which may obscure small masses \\
BI-RADS d & Extremely dense, which lowers the sensitivity of mammography \\
\hline
\end{tabular}
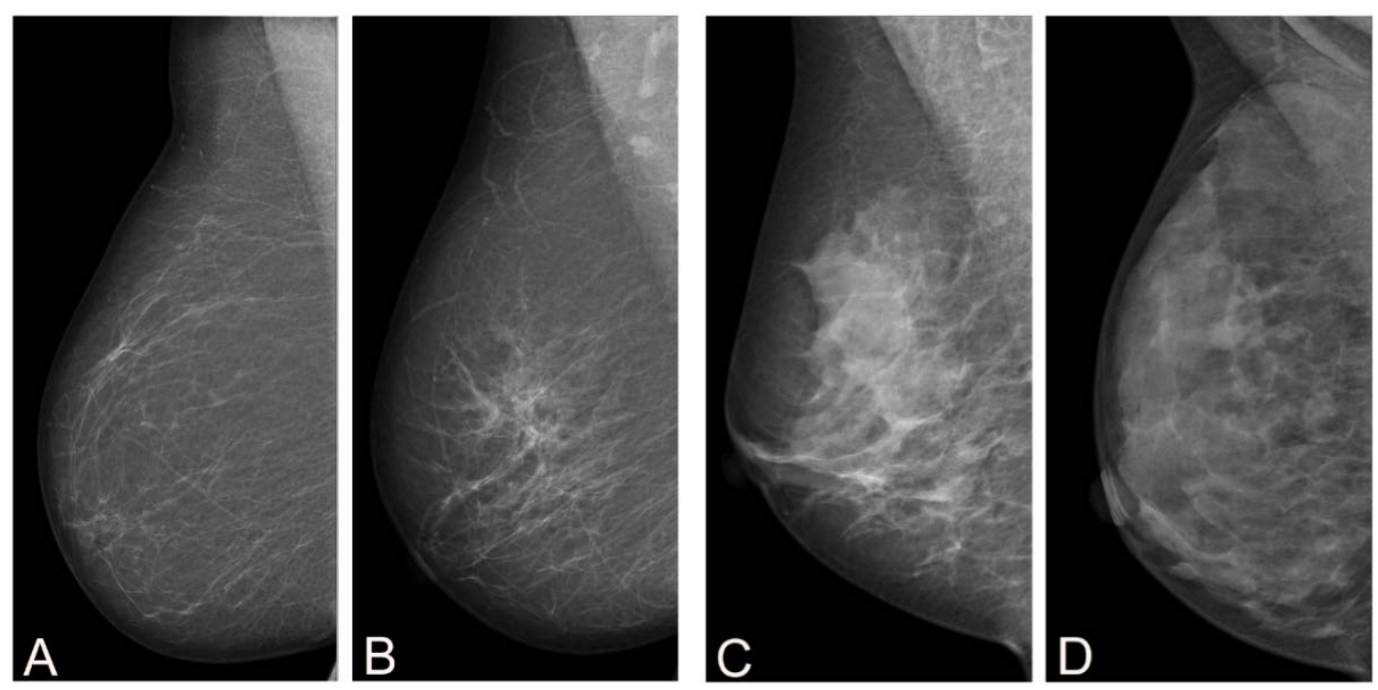

Figure 1.11: Mammographic densisty a-d BI-RADS $5^{\text {th }}$ edition.

However, the visual density classification systems are based on the subjective assessment by radiologists, and thus subject to inter-observer variability $(85,88-90)$. To reduce this variability, semi-quantitative or computer assisted methods were developed, and later fully automated methods that can be used prospectively and retrospectively (91). Fully automated methods are free from inter-observer bias and are essential if a stratified risk-based screening based on density is to be applied. Nevertheless, it has also been demonstrated 
that fully automated density assessment tools are not always concordant with each other (92).

\subsubsection{Classification of mammographic findings}

Abnormal mammographic findings include two types of lesions; soft-tissue lesions and calcifications, or a combination. To provide consistency in reporting and communication of findings, and to facilitate studies on associations between mammographic findings and other parameters, systems for standardized reporting and lesion terminology seem important (87, 93, 94).

The most widely used standardized system for classification of mammographic findings, both in screening and diagnostic mammography, is the BI-RADS classification system (86). BIRADS $5^{\text {th }}$ edition provides a lexicon for standard terminology as well as assessment categories to guide follow-up of the examination. The BI-RADS atlas provides classification systems for lesion description for mammography, ultrasound and MRI. In addition to a widespread use in clinical and screening mammography, the standardized classification system for mammographic findings is suitable for research, as it is a well-established system known to most radiologists (95). According to BI-RADS, mammographic findings are classified as a mass, calcifications, asymmetry, architectural distortion or other findings (86, 95), and these lesions are further classified into subgroups according to appearance (Figure 1.11 and 1.12).

The different subgroups are associated with various potentials for malignancy. A mass is a convex, space-occupying lesion visible in both planes; irregular, spiculated masses are usually associated with malignant lesions, whereas a round or oval and circumscribed lesion is most often benign. Calcifications are classified as either benign or suspicious. The probability of malignancy is substantially higher in fine linear/branching calcifications with a linear or segmental distribution compared with amorphous and diffuse/regional calcifications (86). An asymmetry is a non-space-occupying density, either one-plane (asymmetry) or twoplane (focal asymmetry). A focal asymmetry that is new, larger or more demarcated than before, is called a developing asymmetry, which is the subtype of asymmetries most frequently associated with malignancy (96). One-plane asymmetries usually represent patchy normal fibroglandular tissue with a low risk of malignancy. An architectural distortion is a lesion in which the normal parenchymal pattern of the breast tissue is distorted, usually presenting as a spiculated lesion lacking a central mass. 

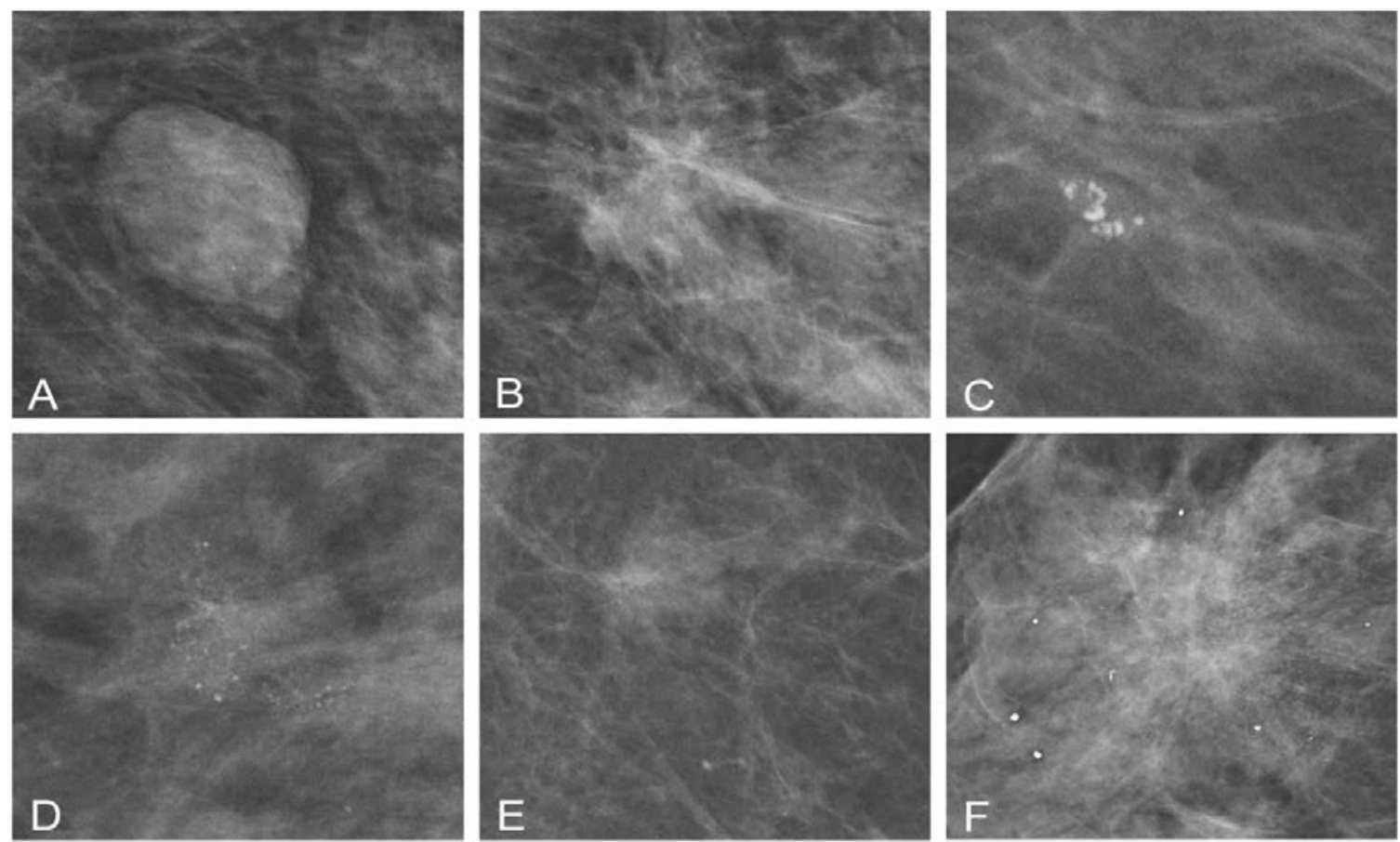

Figure 1.12: Mammographic findings (BI-RADS $5^{\text {th }}$ edition). (A) Oval circumscribed mass; (B) irregular spiculated mass; $(C)$ grouped coarse heterogeneous calcifications; $(D)$ fine pleomorphic calcifications; (E) asymmetry; (F) distortion.

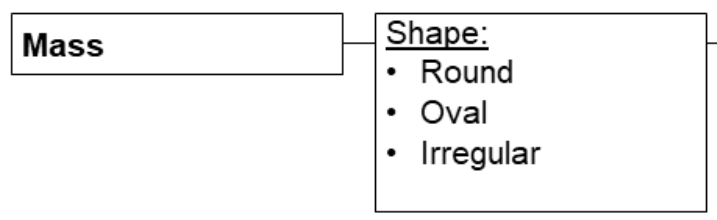

\begin{tabular}{|l|}
\hline Border: \\
\hline - Circumscribed \\
- Obscured \\
- Indistinct \\
- Spiculated \\
\hline
\end{tabular}

\begin{tabular}{|l|l|}
\hline Calcifications & Benign \\
\cline { 2 - 2 } & Suspicious \\
\hline
\end{tabular}

\begin{tabular}{|l|l|}
\hline Asymmetry & $\begin{array}{l}\cdot \text { Asymmetry } \\
\cdot \text { Focal asymmetry } \\
\cdot \text { Global asymmetry } \\
\cdot \text { Developing } \\
\text { asymmetry }\end{array}$ \\
\hline
\end{tabular}

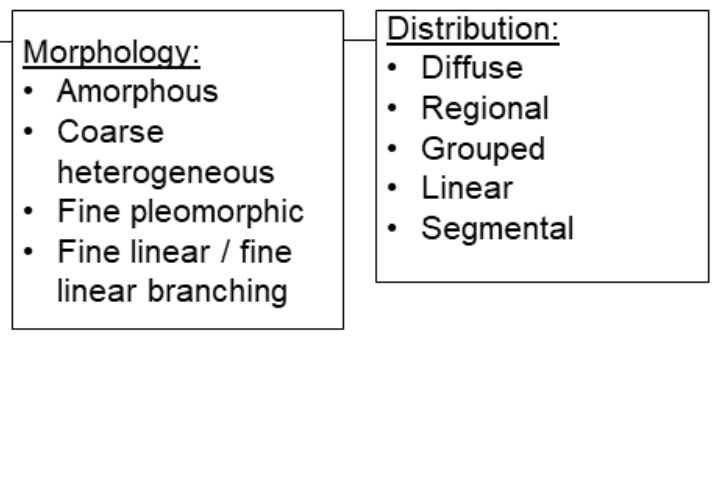

Distortion

Other findings

Figure 1.12. Classification of mammographic findings according to BI-RADS $5^{\text {th }}$ edition. 


\subsection{Breast cancer screening}

\subsubsection{The principles of screening}

Three perspectives are associated with prevention of cancer. Primary prevention is aimed at preventing the occurrence of cancer, secondary prevention is aimed at mortality reduction from the disease, and tertiary prevention is aimed at reducing morbidity of the disease $(3,4)$. Even though several risk factors associated with breast cancer are identified, the exact cause is unknown, and the strongest risk factors, as age, genes, mammographic density and prior history of breast cancer, are hardly available for modification. Thus, primary prevention of breast cancer is challenging.

Screening with mammography is considered secondary prevention, aiming at detecting cancer sufficiently early to reduce mortality from the disease. In addition, early diagnosis may reduce morbidity through less extensive and burdensome treatment $(4,97,98)$.

Wilson and Jungner established ten criteria in the late 1960s to guide the implementation of screening programs (99). Further, the Norwegian Directorate for Health added 6 supplementary criteria for Norwegian screening programs in 2014 (100) (Table 1.3).

Table 1.3. The Wilson Jungner criteria for implementation of screening with additional criteria by the Norwegian Directorate for Health $(99,100)$.

The Wilson Jungner criteria for implementation of screening (1968)

1 The condition sought should be an important health problem

2 There should be an accepted treatment for patients with recognized disease

3 Facilities for diagnosis and treatment should be available

4 There should be a recognizable latent or early symptomatic stage

5 There should be a suitable test or examination

6 The test should be acceptable to the population

7 The natural history of the condition, including development from latent to declared disease, should be adequately understood

8 There should be an agreed policy in whom to treat as patients

9 The cost of case-finding (including diagnosis and treatment of patients diagnosed) should be economically balanced in relation to possible expenditure on medical care as a whole

10 Case-finding should be a continuing process and not a "once and for all" project

Additional criteria by the Norwegian Directorate of Health (2014)

\begin{tabular}{ll}
\hline 11 & The health benefits should outweigh the harms \\
12 & The protection of personal privacy and adherence to the law be ensured \\
13 & The program should be ethically acceptable \\
14 & $\begin{array}{l}\text { Information about participation should be evidence-based and empower making an informed } \\
\text { choice about participation }\end{array}$ \\
15 & The program should be cost-effective \\
16 & There should be a plan for program administration, quality assurance and evaluation \\
\hline
\end{tabular}


Randomized trials for breast cancer screening with mammography were performed during the 60's, 70's and 80's in the United States, Sweden, Canada and the United Kingdom, and these trials constituted the knowledge base for implementation of mammographic screening in several countries $(97,101)$. Over the past $20-30$ years, mammographic screening has become widespread over most developed countries (4). Mammographic screening may be organized as a service screening (population based), or individually referred screening. Organized service screening facilitates systematic reporting and registration which is essential for research based evaluation, quality assurance and improvement; this might be less feasible in more unorganized screening (102). Service screening is widely implemented in several European countries, including Scandinavia, United Kingdom, the Netherlands, Spain and Italy, as well as in Canada, Australia and New Zealand. Mammographic screening in the United States is mainly individually/referred, though with several elements commonly seen in organized service screening (4). Both the International Agency for Research on Cancer (IARC) expert working group as well as the European Commission has recommended service screening to be offered women 50-69 years $(4,23)$.

\subsubsection{BreastScreen Norway}

BreastScreen Norway started in 1996 as a pilot mammographic screening program in four counties, spread gradually and became nationwide in 2005 (3). The program invites all women aged 50-69 to biennial mammographic screening. Screening examination is performed at 26 stationary and 4 mobile screening units, and interpretation, recall assessment and treatment are performed at 17 specialized breast centers. Standard mammographic technique in BreastScreen Norway is two-view digital mammography (DM). Digital breast tomosynthesis (DBT) has been tested as the screening technique in four studies within BreastScreen Norway; the Oslo Tomosynthesis Screening Trial (OTST) (60), the Oslo-Vestfold-Vestre Viken (OVVV) study (103) and the two Tomosynthesis in Bergen (To-Be) trials, To-Be 1(64) and To-Be 2 (104). However, as of today, DBT is not an accepted screening technique outside studies in BreastScreen Norway. To implement new tools or techniques in screening programs in Norway, an independent group have to evaluate the pros and cons and give a recommendation (105). DBT as a screening tool was evaluated in 2017 and the group concluded that the evidence was insufficient to recommend implementation (106).

The screening procedure includes individual screen reading by two dedicated radiologists, who score each breast on a five point scale, which is a modified BI-RADS assessment scale (Table 1.4). 
Table 1.4. Assessment categories in BreastScreen Norway.

\begin{tabular}{ll}
\hline 1 & Normal/benign \\
2 & Probably benign \\
3 & Indeterminate benign/malignant \\
4 & Probably malignant \\
5 & Malignant \\
\hline
\end{tabular}

All exams scored $\geq 2$ by either radiologist are discussed in a consensus meeting to decide whether to recall the woman. The major task in screen-reading is detecting mammograms with suspicious findings in need of further diagnostic work-up and to rule out negative mammograms; the purpose of screening is not definite diagnostics. At recall assessment, the women undergo triple assessment as described in section 1.3.3, alternatively only supplemental imaging if considered adequate. A needle biopsy is performed if indicated. If surgical biopsy or surgical/oncological treatment is indicated, the woman is referred to the local breast surgeons and/or breast oncologists for further evaluation and treatment. Local pathologists examine the biopsy and surgical specimens. Multidisciplinary collaboration (radiologists, surgeons, pathologists and oncologists) is a mainstay in the activity at the breast centers. Data from recall assessment and treatment are continuously registered electronically in the national screening database by the involved professionals.

The Cancer Registry of Norway administers and organizes BreastScreen Norway, and is responsible for among other things, quality assurance of the program. The Cancer Registry collects and ensures the quality of the registered data from the screening invitations and examinations. The data are stored in a national screening database $(3,107)$, and includes over 200 unique variables about the participants' screening history, diagnoses and follow-up. According to the quality assurance manual (108) of BreastScreen Norway, these data should be used for ongoing research, quality assurance and quality improvement of the program. The program continuously provide feedback on screening performance measures, both to the individual radiologists as well as the breast centers. Further, a large number of studies have been conducted and are continuously conducted to evaluate different aspects of the program for the purpose of quality assurance and improvement. Information about the program, its results and research is also communicated to the public as well as to the breast centers and radiologists via the program's website (109). 


\subsubsection{Biases in screening}

The main goal of screening is early diagnosis of the cancer before clinical symptoms arise (98). The time from the tumor is detectable on mammograms until it is clinically detectable represents the preclinical period and is called the sojourn time. As cancers have different growth rates, the sojourn time vary (Figure 1.13). The time span from the tumor was actually detected at screening to the tumor would have been clinically detectable, is referred to as lead-time. Thus, when evaluating survival, the survival time will be longer if the tumor is screen-detected rather than clinically detected due to increased lead time, which represent a bias referred as lead-time bias. As a result, mortality rather than survival is preferred when evaluating efficacy of mammographic screening $(4,98)$.

Slow growing tumors have a longer sojourn time compared with highly aggressive tumors, which may lead to increased detection of slow growing tumors at screening. Slow growing tumors usually have a better prognosis and reduced mortality compared with more aggressive. This may result in a bias in favor of screening, referred to as length-bias $(4,98)$.

A third bias often referred to in screening is the selection bias, which means that the women who attend screening may differ from those who choose not to attend (self-selection). Women attending screening may be more health-conscious or be at a different risk for breast cancer compared with women who do not attend screening. However, results from literature are inconsistent on direction, and the direction of self-selection may vary across different populations $(4,98,110)$.

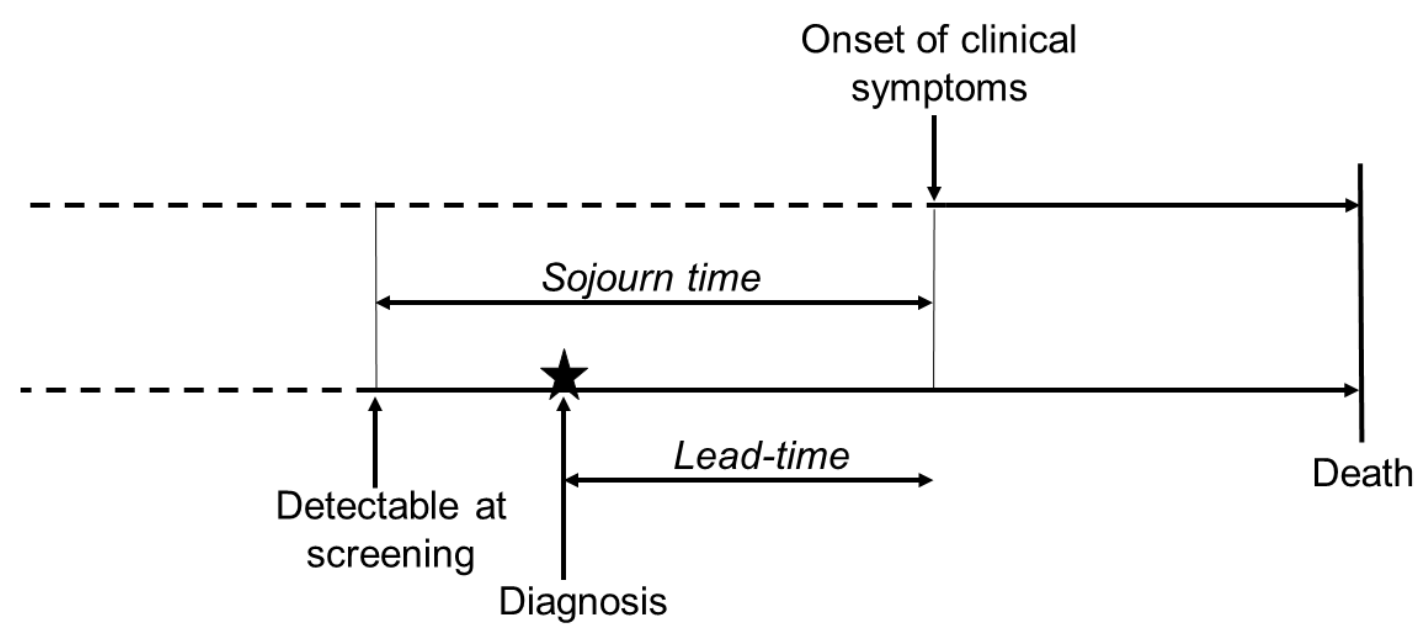

Figure 1.13. Sojourn time and lead-time bias. Sojourn time is the time interval from the cancer is detectable at screening to onset of clinical symptoms, and represents thus the potential gain in early detection by screening. Lead-time is the time interval between detection and onset of clinical symptoms and represents the actual gain in early detection by screening. 


\subsubsection{Effects of mammographic screening - mortality reduction, false positives and overdiagnosis}

Estimation of mortality reduction in mammographic screening is not straightforward; factors that may influence the estimates and need to be considered include background incidence, improvement in treatment over time, follow-up time, concurrent opportunistic screening, characteristics of comparison group (historical or contemporary) as well as whether the estimates are based on individual-level data or population-level or aggregate data. The methodology is not uniform across studies, and differences exist between screening programs regarding target age, screening interval and screening logistics. Thus, the estimated mortality reduction is subject to great variability across studies $(4,97)$. A review considering European studies on breast cancer mortality and mammographic screening until 2011 (111), concluded on an estimated breast cancer mortality reduction of $25-31 \%$ for women invited to screening, and $38-48 \%$ for women actually screened, compared with a non-screened population. Two Norwegian studies demonstrated a reduction in breast cancer mortality of $43 \%$ for screening attendees compared with non-attendees (112), and $28 \%$ for women invited to screening compared with non-invited (113), respectively. However, the mortality reduction from implementation of screening is complicated by a simultaneous improvement in breast cancer treatment. A recent study from Norway estimated a $20 \%$ reduction in breast cancer mortality attributed to invitation to screening and another $23 \%$ reduction due to improved breast cancer treatment. A further 10-15\% mortality reduction was expected for those actually attending screening (5).

Despite a beneficial mortality reduction, negative effects of screening exist, among which false positive screening results and overdiagnosis are the most significant. A false positive screening result is a recall for further assessment due to abnormal mammographic findings that does not result in a diagnosis of screen-detected breast cancer. A false positive screening result may have negative psychological impact on the woman, though these effects have been demonstrated to be mainly transient $(114,115)$. Further, additional tests and even biopsies may also potentially affect her negatively. Finally, a false positive screening result lead to unnecessary costs and use of resources for the health care provider and society (1). In BreastScreen Norway, the cumulative risk for a false positive screening result after 10 screening rounds (20 years) was $20 \%$, whereas the cumulative 20-years' risk for an invasive procedure with benign result was $4.1 \%$ (116). Similar results have been observed in other European screening programs $(117,118)$. An up to 3-fold increased risk of a later diagnosis of screen-detected or interval cancer has been demonstrated in women with a false positive screening result (119-122). Comparison with prior images when interpreting 
the current screening examination may reduce the rate of false-positive recall, and increased knowledge and experience among screen-readers has the potential to increase specificity $(123,124)$.

The most serious and most debated disadvantage of mammographic screening is overdiagnosis. The term refers to screen-detected cancers that would never have been diagnosed clinically during the woman's lifetime in the absence of screening (4), which means that lead time is longer than the woman's remaining lifetime (125). Thus, a cancer diagnosed in a woman who dies from other causes shortly after diagnosis of a screendetected breast cancer may be classified as overdiagnosed, even if the cancer would have been detected clinically and turned out to be lethal in a woman with a normal life expectancy. However, overdiagnosed cancers considered as "harm" in the screening context, usually refer to cancers with no lethal potential to the woman during a normal post-screening lifetime, usually because they are slow growing and/or less aggressive. Nevertheless, the individual screening cancers are not labelled whether they are potentially lethal or potentially overdiagnosed. Thus, a challenge to screening is to optimize and increase early detection to reduce mortality, while simultaneously ensure that the increased detection represents clinically significant or potentially lethal cancers rather than overdiagnosis.

Estimation of overdiagnosis is challenging and vary across studies. Factors that need to be taken into account when estimating overdiagnosis include background breast cancer incidence, adjustment for lead time and length of follow-up. In addition, calculation methods vary. Several studies have been performed, trying to estimate overdiagnosis. In a literature review by the EUROSCREEN group of 13 primary studies, the reported unadjusted estimates in the reviewed studies ranged from $0 \%$ to $54 \%$, but they concluded that the most plausible estimates ranged 1-10\% (126). Other reviews reported estimates of overdiagnosis in a range $11-30 \%(97,101,127-129)$. However, rather than focusing on the estimate itself, attention to the fact that different study populations and methods yield different estimates, and that consensus is hard to achieve, seems to be a reasonable consideration $(130,131)$.

Still, the justification of mammographic screening is widely debated, mainly due to the diverging results and methodologies in studies of mortality reduction and overdiagnosis, as well as the impact of increased breast awareness and improved treatment $(101,132)$. However, whether the studies are based on individual or aggregated data, and whether the data in the balance sheet are derived from studies with comparable study populations and methods used, is crucial when balancing benefits and harms.

Hence, the negative effects of mammographic screening as false positive screening results and overdiagnosis, are considered outweighed by the beneficial effects of early diagnosis on 
mortality reduction and less aggressive treatment by the European Commission, the International Agency for Cancer Research, the World Health Organization as well as several American cancer organizations (4, 97, 133-135).

\subsubsection{Challenges in mammographic screening}

In studies, sensitivities of mammography as screening method have been reported ranging from $68 \%$ to $88 \%$ and specificities from $82 \%$ to $98 \%$ (136). The sensitivity of mammography is lower in women with high mammographic density, see also chapter 1.4.4, due to the masking effect of tissue overlap on lesions that hampers lesion detection. However, masking of lesions may occur at all densities, if the lesions have similar density as breast tissue. Overlap of tissue structures may also create "pseudo-lesions" resembling real lesions. To overcome these limitations, and thereby improve early detection of cancer in screening, different strategies may be applied. Such strategies may include development of novel imaging techniques, as DBT, as well as offering supplementary screening techniques to women with dense breasts, as ultrasound and MRI. Due to the potential reduction of the masking effect of dense breast tissue by DBT, there were great expectations to DBT as a screening tool in dense breasts. However studies have demonstrated little impact on cancer detection by DBT in women with the highest mammographic density category (137-139). DBT in screening is further discussed in the next chapter.

Another challenge in mammographic screening is interval cancer. Interval cancer represents about $25 \%$ of the cancers detected among screening attendees, and is defined as cancer diagnosed between two scheduled screening rounds after a negative screening episode with or without recall assessment $(23,140)$. In some interval cancers visible mammographic findings are identified when reviewing prior screening mammograms, thus earlier diagnosis might have been possible. Interval cancer is discussed in more detail in chapter 1.5.7.

An important mainstay in mammographic screening is the screen-readers and their performance. Screening mammograms are mainly read by radiologists. However, the requirements to qualify as a screen-reader vary across programs. As most screening mammograms are negative, a certain reading volume might be necessary to achieve adequate experience in screen-reading, though associations between reading volume and performance vary in studies. Many screening programs state recommendations or requirements for annual reading volume. However, this volume vary, from 960 readings per two years in the United States to an annual reading volume of 5000 mammograms in the United Kingdom (141-144). In a study from BreastScreen Norway, an annual screening volume between 4000-10 000 screen-readings was considered to give the most optimal 
screening performance (141). In addition, time and length of the screen-reading session may impact radiologists' performance by influencing attention and vigilance (145).

The extent of organized training and regular audits with feedback to the readers and programs vary widely between programs, though these factors have been demonstrated to positively impact reader sensitivity and the proportion of false positives (142, 143, 146-151). Platforms that provide mammographic test-sets and produce interactive feedback to the radiologists, have been developed; examples are PERFORMS, and BREAST which are currently in use in the United Kingdom and Australia, respectively (142). Additionally, regular audits and reviews of mammograms from women diagnosed with screen-detected and interval cancer may increase performance of the screen-readers, by increasing awareness of potential pitfalls in screening.

\subsubsection{DBT in screening}

Several retrospective and prospective studies, including randomized controlled trials (RCTs), have compared outcome of screening with DBT versus DM $(39,45,56-65)$. The majority of the studies have demonstrated an increased cancer detection with DBT, but the effect on recall rates varied (Table 1.5). The retrospective studies were mainly unpaired, comparing different cohorts screened with either DBT or DM and were conducted in the United States. Most non-randomized prospective European studies were paired, in which the same women were screened with both DM and DBT+DM/SM. Hence, the same women were included in both the DM and the DBT group, with sequential reading by the same or different readers. Recall routines for further assessment varied in the paired studies, e.g. in the Screening with Tomosynthesis OR standard Mammography (STORM) trials, all women recalled by the readers in either part were recalled, while the Oslo Tomosynthesis Screening Trial (OTST) and the Malmö Breast Tomosynthesis Screening Trial (MBTST) made a consensus-based decision whether to recall, but with all images available, including the DBT images. The RCTs represent the least biased study design. As of today two RCTs have been conducted; the Tomosynthesis in Bergen (To-Be) 1 trial and the Reggio Emilia Tomosynthesis Randomized (RETomo) trial. However, additional large randomized trials are now recruiting both in the United States (152) and United Kingdom (153).

A meta-analysis by Marinovich et al (154) compared results from 4 paired prospective and 11 unpaired retrospective studies, and demonstrated a total pooled incremental cancer detection rate of $1.6 / 1000$ for screening with DBT versus DM $(p<0.001)$. Regarding recall rate, a total absolute non-significant decrease of $-2.2 \%$ was observed for DBT versus DM: however, an even higher absolute reduction of $-2.9 \%$ was observed for unpaired studies 
$(p<0.001)$, whereas an absolute increase of $0.5 \%$ was observed for paired studies (not significant). A major difference between the unpaired and paired studies in this meta-analysis was that all unpaired studies were conducted in the United States, whereas the paired studies were conducted in Scandinavia/Europe. The screening logistics in the United States differs from European screening programs, with mainly single-reading and annual mammography compared to double reading and biennial screening in the European studies. Further, the recall rates in the American studies were markedly higher than in Europe prior to implementation of DBT. Thus, screening logistics and baseline recall rate might have influenced the recall rates with DBT more than the paired/unpaired study design as such. This is also demonstrated in the studies included in table 1.5; the recall rate in the studies from the United States (56-58) are higher both for DBT and DM compared with the other, European studies.

DBT-systems offered by different vendors vary in technical approach, including filter/detector material, x-ray tube motion, scan angle and reconstruction algorithms. Whether or how these differences might impact cancer detection is unclear, but differences in outcome between vendors may exist (155). The vast majority of studies on DBT in screening have been performed with equipment from Hologic, but equipment from Siemens and GE was used in some European studies, including the RCTs (Table 1.5).

Table 1.5. Study design, cancer detection rate and recall rate in studies comparing screening with DBT and standard DM. Cancer detection rates are given per 1000 screened, and recall rates as percentages.

\begin{tabular}{|c|c|c|c|c|c|}
\hline \multirow[b]{2}{*}{ Study } & \multirow[b]{2}{*}{ Design } & \multicolumn{2}{|c|}{ Cancer detection rate } & \multicolumn{2}{|c|}{ Recall rate } \\
\hline & & DBT & DM & DBT & $\mathrm{DM}$ \\
\hline Romero Martin $2018(61)^{1}$ & Prospective, paired & 5.7 & 4.7 & 4.4 & 5.0 \\
\hline Ciatto $2013(62)^{1}$ & Prospective, paired & 8.1 & 5.3 & $\ldots$ & $\ldots$ \\
\hline Bernardi 2016 (45) ${ }^{1}$ & Prospective, paired & 8.5 & 6.3 & $\ldots$ & $\ldots$ \\
\hline Zackrisson 2018 (39) ${ }^{2}$ & Prospective, paired & 8.7 & 6.5 & 3.6 & 2.5 \\
\hline Skaane $2013(60,156)^{1}$ & Prospective, paired & 8.0 & 6.1 & 3.4 & 2.6 \\
\hline Caumo $2018(63)^{1}$ & Prospective, unpaired & 9.3 & 5.4 & 4.0 & 4.2 \\
\hline Bernardi $2020(157)^{1}$ & Prospective, unpaired * & 8.7 & 5.5 & 2.6 & 3.2 \\
\hline Hofvind $2019(64)^{3}$ & Randomized controlled study & 6.6 & 6.1 & 3.1 & 4.0 \\
\hline Pattacini $2018(65)^{3}$ & Randomized controlled study & 8.6 & 4.5 & 3.5 & 3.5 \\
\hline Friedewald 2014 (58) ${ }^{1}$ & Retrospective, unpaired & 5.4 & 4.2 & 9.1 & 10.7 \\
\hline Greenberg $2014(56)^{1}$ & Retrospective, unpaired & 6.3 & 4.9 & 13.6 & 16.2 \\
\hline Conant $2016(57)^{1}$ & Retrospective, unpaired & 5.9 & 4.4 & 8.7 & 10.4 \\
\hline
\end{tabular}

* Historical cohort with DM. ${ }^{1}$ Hologic; ${ }^{2}$ Siemens; ${ }^{3} \mathrm{GE}$.

Studies have demonstrated increased reading time for screening with DBT versus DM. In the two Norwegian studies reading times were almost doubled with DBT (mean reading time in 
OTST 89 s versus 48 s; To-Be 66 s versus 39 s) (42, 158). Similar results were also demonstrated in other European studies $(59,61,65)$. However, as the radiologists' experience with DBT screen reading increases, the reading time might be reduced.

When an increase in cancer detection at screening is observed, a decrease in interval cancer rate is anticipated. To date, among the few studies reporting interval cancer rates after screening with DBT+DM/SM, only MBTST has demonstrated a statistically significant interval cancer reduction, otherwise no statistically significant differences were observed (Table 1.6) $(104,157,159-165)$. A recent meta-analysis of five European studies demonstrated a pooled difference in interval cancer rates for DBT versus DM of -0.15/1000 (95\% Cl -0.590.29) (166). Additionally, in an individual participant data meta-analysis of four prospective studies, including 66451 screening examinations with DBT and 153800 with DM, no statistically significant difference in interval cancer rates were observed for screening with DBT versus DM (167).

Table 1.6. Interval cancer rates per 1000 screened for studies comparing screening with DBT with DM.

\begin{tabular}{lcccccc} 
& \multicolumn{2}{c}{$\begin{array}{c}\text { Interval cancer rate } \\
\text { DBT }\end{array}$} & & \multicolumn{2}{c}{$\begin{array}{c}\text { Interval cancer rate } \\
\text { DM }\end{array}$} \\
\cline { 2 - 3 } \cline { 5 - 6 } Study & $\mathrm{n}$ & per 1000 & & $\mathrm{n}$ & DM \\
\hline Bernardi 2020 (157) & 51 & 1.1 & & 51 & 1.4 \\
Houssami 2018 (159) & 9 & 1.2 & & 40 & 1.6 \\
Skaane 2018 (160) & 51 & 2.1 & & 118 & 2.0 \\
Johnson 2021 (165) & 21 & 1.6 & & 76 & 2.8 \\
Hofvind 2021 (104) & 20 & 1.4 & & 29 & 2.0 \\
Bahl 2018 (162) * & 84 & 1.1 & & 83 & 1.1 \\
McDonald 2016 (164) * & $\ldots$ & 0.5 & & $\ldots$ & 0.7 \\
Winter 2020 (163)* & 40 & 0.3 & & 35 & 0.3 \\
\hline
\end{tabular}

${ }^{\star}$ Annual screening.

Further, no unanimous results exist regarding which cancers are detected with DBT. Is the increased cancer detection with DBT only a prevalent effect, due to an increase in lead time? Or do DBT detect other cancer types than DM? This is an important aspect, as one of the main interests when introducing new screening techniques is whether the new technique is able to increase detection of clinically significant cancers that have the potential to be "killing cancers". Studies have demonstrated somewhat diverging results regarding histopathological tumor characteristics for cancers detected with DBT versus DM; some studies observed no differences between groups $(39,63,64)$ whereas others demonstrated a higher detection of smaller tumors of lower histological grade with DBT compared with DM $(61,65)$. Hence, further studies were needed (155). 
As of April 2021, screening with DBT is only allowed in BreastScreen Norway as part of studies, due to an evaluation in 2017, as described earlier (106). In the United States, screening with DBT has become widespread over the past decade (155), whereas in Europe, most programs have not implemented DBT as routine screening method, although widely used in clinical settings. The European Commission Initiative on Breast Cancer (ECIBC) recommends that for asymptomatic women with an average risk of breast cancer, either digital breast tomosynthesis (DBT) or digital mammography (DM) should be used in the context of an organized screening program. The recommendation is conditional for either DBT or DM, and the certainty of the evidence is considered very low (133).

\subsubsection{Interval breast cancer}

\subsubsection{Definition, rates and incidence}

Interval breast cancer is defined by the European Guidelines (23) as "A primary breast cancer diagnosed in a woman who had a screening test, with/without further assessment, which was negative for malignancy in a time period equal to a screening interval and after a normal mammography, either before the next invitation to screening or within a time period equal to a screening interval for a woman who has reached the upper age limit for screening".

Interval cancer is inevitable in a population based screening program, though not caused by the program itself, as it is a symptomatic cancer that would have appeared regardless of participation in screening. Still, effort should be made to keep the rates as low as possible, both to obtain a high quality program as well as to maintain trust in the program among women and society. The incidence of interval cancer in a breast cancer screening program is an indicator of the effectiveness of the program. High rates of interval cancer may impair the program's ability to reduce breast cancer mortality, as interval cancers generally have unfavorable prognostic and predictive tumor characteristics. Further, the interval cancer rates are essential in assessing the program sensitivity and evaluation of radiologists' performance $(140,168)$.

The interval cancer rates vary across screening programs $(169,170)$, and it is estimated that interval cancer accounts for $17-30 \%$ of all cancers in biennial screening programs (171). A review article reporting interval cancer rates in several studies reported interval cancer rates ranging from 0.8 to 2.1 per 1000 screened in biennial screening programs (171). Further, interval cancer rates were higher in subsequent than prevalent screening.

The completeness of registration of interval cancers vary across programs, and is a major determinant of interval cancer rates. A prerequisite for proper interval cancer registration is a 
nationwide cancer registry, as the interval cancer may be diagnosed elsewhere than the screening exam was performed. Further, a precise linkage between the data in the cancer registry and the screening data is essential (168). In BreastScreen Norway, the rate of interval cancer between 1996 and 2016 was relatively stable, 1.8 per 1000 women, comprising $24 \%$ of all cancers among the participants in the screening program (3). BreastScreen Norway is considered almost $100 \%$ complete as regards interval cancer registration, and the linkage makes it possible to follow the woman if she moves within Norway. These factors probably explain the relatively high interval cancer rates in Norway compared with other countries (172).

Other factors influencing the interval cancer rates are the background incidence of breast cancer, the length of the screening interval and the organization of the program. In programs with biennial screening, like most European screening programs, the rates of interval cancer are usually higher than in programs with annual screening (as in the United States), and lower than in screening programs performing screening every third year, as in the United Kingdom (171). The occurrence of interval cancer is higher in the second than the first year in biennial screening and even higher in the third year with triennial screening which adds to the effect of the screening interval (173-178). Double-reading of mammograms is demonstrated to reduce the interval cancer rates compared with single-reading (179). In addition, the expected or accepted recall rate in the program may influence the incidence of interval cancer; by accepting a higher rate of recall, a higher number of subtle findings which otherwise may develop and be diagnosed as interval cancer - might be recalled for further assessment compared with a program in which very low recall rates are anticipated $(180,181)$. However, a modelling study demonstrated that an increase in recall rate above approximately $7 \%$ resulted in only a slight increase in detection of low grade non-invasive cancers and no further increased detection of invasive and high grade non-invasive cancers (182).

Even if the definition above by the European Guidelines is widely used, the interpretation of what counts as an interval cancer vary among different screening programs and radiologists, as do the calculation methods, thus influencing the calculated rates of interval cancer in a program $(168,176)$. Whether the definition of interval cancer includes both DCIS and invasive cancer, or solely invasive cancer is significant, as is also the classification of cancer detected at short-term follow-up. Some may advocate that a cancer detected at a follow-up 6 months after screening recall should be classified as a delayed screen-detected cancer, whereas others may define the cancer as an interval cancer. A frequent use of short-term follow-up, as in the BI-RADS 3 assessment category (86), will influence the interval cancer 
rate $(121,168)$. However, a short-term follow-up is not approved by the Norwegian guidelines (108).

\subsubsection{Causes and characteristics of interval cancer}

The majority of interval cancers are diagnosed due to clinical symptoms, while some interval cancers are diagnosed incidentally in other radiological examinations for non-breast related symptoms, as CT, PET or MRI (183). In addition, some asymptomatic women have a mammogram in the inter-screening interval as part of a high-risk surveillance program or simply because they want ("opportunistic screening"). Factors associated with an increased risk for interval cancer are dense breasts, low body-mass index, a family history of breast cancer, use of hormone replacement therapy and a previous false-positive screening exam $(121,171,184-186)$.

Some interval cancers are not even in retrospect mammographic visible at the time of screening, but grow rapidly and become detectable in the inter-screening interval. In other interval cancers, abnormal findings are retrospectively visible at review of prior mammograms, ranging from very subtle or benign appearing findings to more obvious findings, with a suspicious or even typically malignant appearance. Cases with obvious findings on prior mammograms can be considered false negative or missed. These cancers are either not perceived at all by the screen-readers, or not interpreted as suspicious findings and dismissed at screening or consensus. In a study from BreastScreen Norway, $6.5 \%$ of interval cancers had been discussed in a consensus-meeting at prior screening, though it was not demonstrated whether the discussed finding represented the later interval cancer (187). The estimated proportion of interval cancers considered missed at prior screening vary between studies, but is in general reported $20-25 \%$ (171). Some cancers, approximately $10-$ $15 \%$ (171), are not visible on mammograms at diagnosis nor prior screening; either masked by the breast tissue, or located outside the target area of the mammogram.

The interval cancers have larger tumor diameter, are of higher histological grade, less frequent DCIS, with less favorable predictive molecular subtypes than screen-detected cancer, and with a higher risk of relapse and less favorable survival (188-196). Thus, interval cancer usually represent clinically significant cancer with a lethal potential. Even if numerous studies on interval cancer have been performed, they still occur in all breast cancer screening programs, and knowledge is still needed to reduce the occurrence. Focused points in that respect may include the association of interval cancers with screen-readers' performance, mammographic findings including mammographic density as well as tumor characteristics and screening logistics. In that respect, radiological reviews may be valuable. 


\subsection{Radiological review of prior screening mammograms}

\subsubsection{Purpose}

According to the European Guidelines (23) "Radiologists in each unit must ensure that a suitable mechanism exists for the review and audit of all interval cancers. This review should be an essential part of routine radiological audit, and plays a key role in the continuing medical education of radiologists involved in the programme." By performing reviews, radiologists may gain knowledge to understand why the interval cancers occur, and further improve their awareness, skills and performance in the program (183).

Radiological reviews may be undertaken in different settings. An informal review is usually performed as part of everyday clinical practice, as a case-by-case assessment of prior screening mammograms upon the diagnosis of interval cancer. Further, an organized review of the department's interval cancers may be performed on a regular or irregular basis by the individual radiologists or a panel of colleagues. Conduction of larger research-based review studies is an approach to provide evidence-based knowledge about different types of interval cancer that may contribute to a reduction of interval cancers in screening $(171,183)$.

Likewise, radiological reviews of the prior screening mammograms may also be performed for screen-detected breast cancer, to explore whether an even earlier diagnosis was possible. However, few radiological review studies of screen-detected cancer are reported, and as for interval cancers, knowledge about the association between mammographic findings and review classification categories is limited.

\subsubsection{Classification}

At the review, the cancers are classified according to whether there were visible abnormalities at the eventual cancer site on mammograms from the prior screening exam. No standardization of classification exists, but the cancers are usually classified into either of these categories (Table 1.7):

True/true negative cancers: These are cancers that were not visible on the most recent prior screening mammogram; the prior mammogram was true negative, meaning that the cancer has become detectable within the inter-screening interval.

Missed/false negative cancers: These are cancers that in retrospect were visible on the prior screening mammogram, and a recall is usually considered appropriate in retrospect. Missed cancers are either not perceived at screen-reading, or perceived but not interpreted as sufficient suspicious for further assessment at screen-reading or consensus. 
Minimal signs: If there are visible findings at the eventual cancer site on prior screening mammograms, but the findings are subtle or non-specific and a recall is not necessarily regarded as warranting assessment, the cancer may be classified as minimal signs. The bounding line between the categories missed and minimal signs is not consistent and may be related to the expected recall rates within the program as well as the readers' subjective experiences. This category may be further subdivided into actionable (recall possible) and non-actionable (recall not possible within the limitations of a screening program).

Occult cancers: Some cancers are not visible on mammograms, neither at diagnosis nor prior screening. One explanation is that the tumor is obscured by adjacent structures and overlapping tissues, often caused by dense breast tissue. Another explanation is that the tumor's location is not included in the mammogram, for example in the axillary region, or far medially in the breast.

Table 1.7. Status of mammograms (positive or negative) at prior screening and diagnostic images at review by review category.

\begin{tabular}{lcc} 
Category & Prior screening & Diagnostic images \\
\hline True/true negative & Negative & Positive \\
Missed/false negative & Positive & Positive \\
Minimal signs & (Positive) & Positive \\
Occult & Negative & Negative \\
\hline
\end{tabular}

\subsubsection{Review design}

The European guidelines from 2006 proposes a review process, in which the first step includes a blinded review with provisional classification based on whether there are visible findings on prior screening mammograms, and a second step in which both the screening mammograms and the diagnostic mammograms are taken into account for a final classification (23). However, there is no standardization across programs and studies regarding the review process. Further, studies have shown that different procedures and study designs are highly influencing the results of the review (140). This makes comparison of studies and review processes across programs challenging.

Important factors influencing the review process are the level of information available at review, the level of enriched cancer cases within the batch, number of reviewers and their knowledge about the cancer cases (197-200). In a blinded review, only the screening mammograms are available for the reviewers while informed reviews also make the diagnostic mammograms available. In blinded reviews, prior screening mammograms of cancer cases may be mixed with negative mammograms. The ratio between negative and 
abnormal/pre-interval cancer mammograms and whether the reading batch resembles a normal screening situation or is enriched with abnormal mammograms, is shown to affect the results of the review. Further, the number of reviewers and whether the review is individual or consensus-based may affect the classification (Table 1.8) (197-200). Whether the review is performed by radiologists with personally knowledge about the cancer cases, and whether more than one set of prior screening mammograms are available may also influence the classification. In a study from BreastScreen Norway, the proportion of missed cancer varied from $1.3 \%$ to $35.9 \%$ depending on review design (201).

Table 1.8. Factors affecting review classification categories

Factors affecting review classification categories

\begin{tabular}{ll} 
Study awareness & $\begin{array}{l}\text { Higher proportion of missed cancers if reviewers are aware they } \\
\text { participate in a study }\end{array}$ \\
Level of information & $\begin{array}{l}\text { Blinded, semi-informed or informed? The more information available to } \\
\text { reviewers, the higher proportion of missed cancers }\end{array}$ \\
Case-mix & $\begin{array}{l}\text { Ratio normal to abnormal mammograms. Higher proportion of missed } \\
\text { cancers if all reviewed cases are cancer cases }\end{array}$ \\
Number of reviewers and & $\begin{array}{l}\text { The more reviewers required to classifiy a case as missed, the lower } \\
\text { proportion of missed cancers. }\end{array}$ \\
\hline
\end{tabular}

Several radiological review studies have been performed, mostly based on screen-film mammography rather than digital mammography, which is the standard technique of today (202). Further, no unanimous results exist regarding mammographic findings on prior screening mammograms of missed and minimal signs cancer. Whether or how mammographic density differ among the classification categories is also not fully understood.

\subsection{Improved breast cancer screening for women and society - a radiological approach}

The inherent wish of BreastScreen Norway and associated personnel, to provide the best mammographic screening to the target women, society and other stakeholders, was the driving force for this $\mathrm{PhD}$ project. On the basis of the described challenges in mammographic screening, the well-organized breast cancer screening program in Norway, and the highly dedicated and experienced breast radiologists within the program, the studies were designed. The project focused on ways to improve breast cancer screening, both concerning screening technique and radiologists' performance. Hopefully, results of the studies performed will contribute in filling some of the knowledge gaps. 


\section{Aims of the thesis}

The overall aim of this $\mathrm{PhD}$ project was to investigate ways to improve early detection of breast cancers in BreastScreen Norway. Improve was defined as increased detection of clinically significant, or "killing" cancers. To achieve this overall aim and address the associated knowledge gaps, we performed two studies, one study on digital breast tomosynthesis (DBT) and one radiological review study.

Study 1: DBT as a screening tool for breast cancer - The Oslo-Vestfold-Vestre Viken (OVVV) study

When this PhD project started, a small number of studies had demonstrated that DBT in combination with DM had the potential to increase the rate of screen-detected cancer compared with standard DM alone. Further studies were needed, in particular for screening with $\mathrm{DBT}+\mathrm{SM}$. During the course of this project, more studies reported results, mainly confirming the initial results. However, whether the increased detection of cancer with DBT represented an increase in detection of clinically significant cancers with a lethal potential, or more slow-growing, dormant cancers that potentially increased the burden of overdiagnosis, remained unclear. Further, there was limited knowledge regarding the effect on interval cancer; due to an increased detection of cancer at screening, a decline in interval cancer rate was anticipated. As interval cancers are associated with a higher mortality than screendetected cancers, such decline was desirable.

The OVVV-study aimed to fill knowledge gaps related to these aspects. The study included $>90000$ women screened with either DBT+SM or standard DM, followed for two years for interval cancer and screening outcome in the consecutive screening round.

Primary objectives of study 1 (paper 1 and 2):

To investigate early screening outcome, defined by rates of recall and screen-detected breast cancer, positive predictive value of recall as well as prognostic and predictive tumor characteristics for screening with DBT+SM compared with screening with standard DM. Further, using the same study population, we wanted to investigate rates and prognostic/predictive tumor characteristics of interval cancer after screening with DBT+SM versus DM as well as early screening outcome in the consecutive screening round. 
Study 2: Review of prior screening and diagnostic mammograms from women diagnosed with screen-detected or interval breast cancer

In retrospect, minor or major abnormalities are regularly visible on prior screening mammograms from women diagnosed with interval and screen-detected cancer. These findings represent a potential of earlier diagnosis. Few studies have explored the magnitude of missed cancer for screening with DM, and knowledge about the association with histopathological tumor characteristics is crucial to evaluate the clinical significance of an earlier diagnosis. Further, to increase knowledge and awareness among the screen-readers and thereby possibly reduce the occurrence of missed cancer, classification of the mammographic findings is essential.

To address these knowledge gaps, this PhD project included administration and performance of a radiological review of prior screening and diagnostic mammograms from about 2200 women diagnosed with interval or screen-detected breast cancer. The review was fully informed and consensus based and involved mammograms and radiologists from all breast centers in Norway.

Primary objectives of study 2 (paper 3 and 4):

To classify the reviewed cancer cases according to visibility of findings on prior screening mammograms, and to explore and analyze associations between review classification categories, mammographic findings and histopathological tumor characteristics for screendetected and interval cancer. 


\section{Materials and methods}

\subsection{Study 1 - The OVVV-study}

\subsubsection{Ethics and approvals}

The OVVV-study was an independent study, designed on the basis of the results and power estimations from the Oslo Tomosynthesis Screening Trial (OTST), performed in 2010-11 (60). The Data Protection Official at the Cancer Registry of Norway approved this prospective study (approval number 2013/18023). This also included use of de-identified mammograms as illustrations. The OTST had demonstrated a 30\% increased cancer detection when adding DBT to the screening technique, and further, that the cancer detection for DBT in combination with synthetic mammograms (SM) was non-inferior to the combination of DBT and standard digital mammography (DM). Thus, the OVVV-study was considered as a quality improvement study to provide further data to prepare the ground for implementation of DBT+SM in BreastScreen Norway.

The Cancer Registry Regulation waived the requirement to obtain written informed consent for use of screening data for quality assurance and research (107). Women with negative screening results have the right to request that their screening data be deleted from the Cancer Registry of Norway after the quality of data has been assured. About $1.9 \%$ of all participating women ever have exercised their right to do so (3), and data from these women was not included in the study.

\subsubsection{Study methodology}

The OVVV-study was a prospective, population based cohort study comparing screening with DBT+SM with screening with DM in an ordinary screening setting. The study included all women screened at the three breast centers Oslo, Vestfold and Vestre Viken between February $1^{\text {st }} 2014$ and December $31^{\text {st }} 2015$ (baseline screening). Women had a two-year follow-up for interval cancer and screening results in the consecutive screening round (February $1^{\text {st }} 2016$ - December $31^{\text {st }} 2017$ ).

Due to the results from the OTST as described above, and already installed equipment for screening with DBT+SM in Oslo, women screened in Oslo comprised the study group, and were screened at baseline with DBT+SM (Dimensions; Hologic, Bedford, Mass). Women screened at two nearby breast centers (Vestfold and Vestre Viken) comprised the control group and were screened at baseline with standard DM (Dimensions; Hologic, Bedford, 
Mass [Vestfold] and Mammomat Inspiration; Siemens, Erlangen, Germany or Seno

Essential, GE Healthcare, Buc, France [Vestre Viken]). In the consecutive screening round, all women, both in the study and control group, were screened with DM.

The three breast centers are located in neighboring counties in the southeastern Norway and had comparable early outcome measures (recall rate, cancer detection rate and interval cancer rate) in the pre-study period 2007-2009 (103).

\subsubsection{Study population}

At baseline, 34641 women were included in the study group (Oslo, DBT+SM) and 57763 in the control group (Vestfold and Vestre Viken, DM) (Figure 3.1). The study sample of the consecutive screening round included all re-attending women in the study group ( $n=26474)$ and the control group ( $n=45543)$. Women who did not re-attend included women diagnosed with screen-detected cancer at baseline $(n=679)$ or interval cancer $(n=156)$, women invited but not re-attending ( $n=11248)$ and women not invited because they exceeded the upper age limit $(n=8304)$.

In paper 1, the study population at baseline screening also included women screened in January 2016 ( $n=4$ 853), as the study originally included women screened in one complete screening round of two years. However, when the analyses in paper 2 were performed, data on interval cancer and screen-detected cancer from the consecutive screening round were not available for women screened in January 2016, due to delays in registration of cancer cases in the Cancer Registry's database. Thus, data from the screening examinations of these women were excluded from the analyses in paper 2 and in this thesis. Women with a pre-study history of breast cancer were also excluded from analyses in paper 2 and the thesis $(n=1671)$.

Power estimation and estimation of sample size was based on results from the Oslo Tomosynthesis Screening Trial (60) demonstrating a 30\% increase in cancer detection for DBT. Thus, based on the number of women enrolled in the OVVV-study, we had $80 \%$ power to observe a difference of 0.2 percentage points or more in both recall rate and rate of screen-detected cancer between DBT+SM and DM at a significance level of 0.05 . The study was not powered to prove any differences in interval cancer rate. 


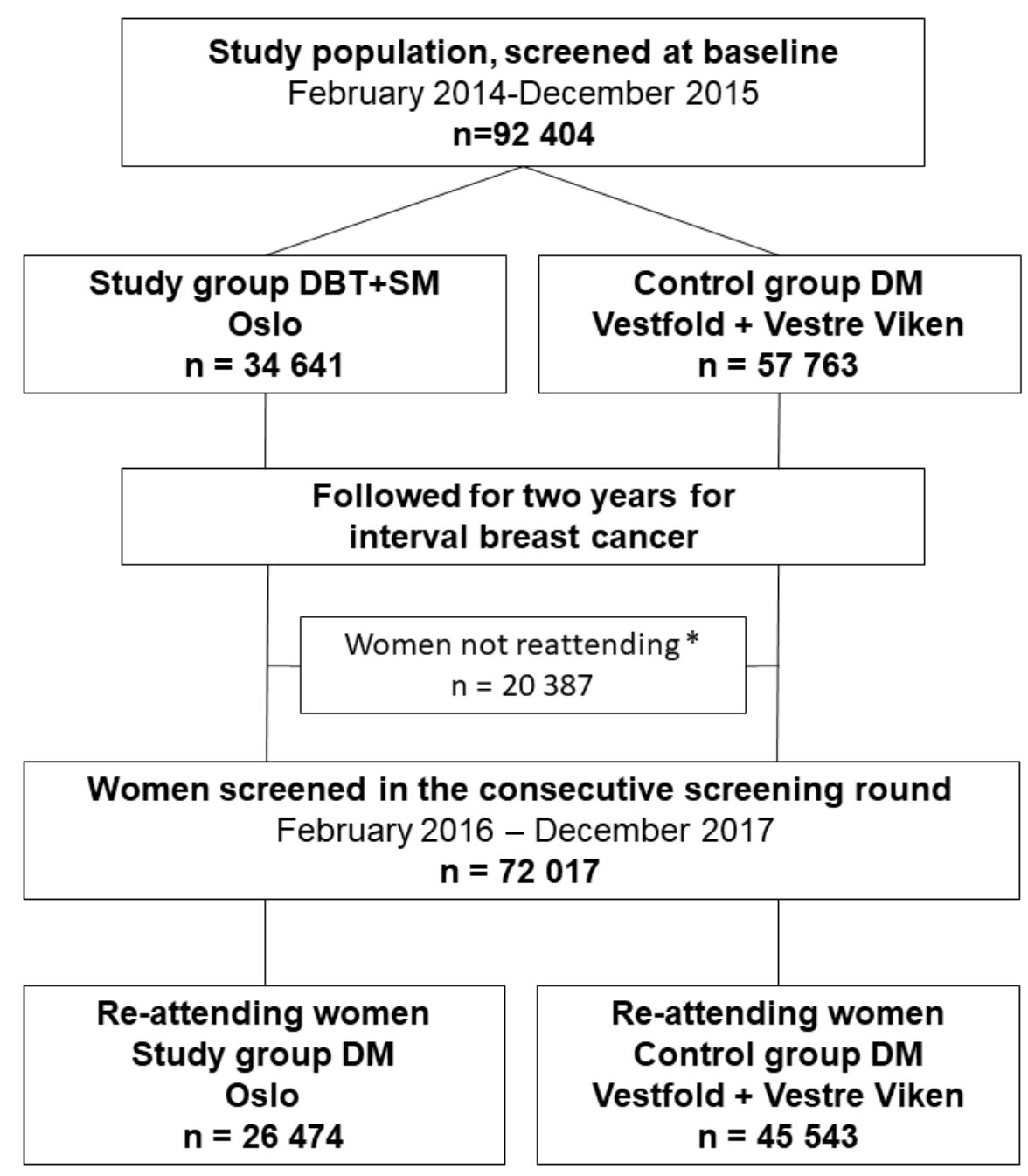

Figure 3.1. OVVV Study sample. * Reasons for not reattending: screen-detected cancer at baseline, interval cancer, invited but not reattending, not invited due to upper age limit.

\subsubsection{Data collection}

The women were screened locally at the screening units in Oslo, Vestfold and Vestre Viken as usual, as a part of BreastScreen Norway. Dedicated breast radiologists at the three breast centers read all screening mammograms locally. The radiologists in Oslo were trained in screen-reading DBT, due to the prior study, OTST. The screening and recall assessment procedure in BreastScreen Norway is described in detail in section 1.5.2. Local pathologists examined the pathological specimens (needle biopsy and/or surgical specimens) of the screen-detected (both baseline and consecutive screening) and the interval cancers. According to standard procedures in BreastScreen Norway, data from screen reading, consensus, recall, interval cancer as well as results from the pathological examinations were registered in the Cancer Registry's database (3). 
During the study period, preliminary study results from screening and recall were regularly reported to the radiologists and pathologists at the breast centers, in written reports and meetings in the project group.

\subsubsection{Data analyses}

The analyses were performed on de-identified data extracted from the Cancer Registry databases. We analyzed early outcome measures, defined as recall rates, rates of screendetected breast cancer, positive predictive value of recalls (PPV-1), rates of interval cancer, sensitivity/specificity of the screening examinations as well as histopathological tumor characteristics using data from baseline, interval cancers and the consecutive screening round.

Recall was defined as recall for further assessment after an abnormal mammographic screening exam, and screen-detected cancer as cancer (DCIS or invasive) detected at recall. Interval cancer was defined as cancer diagnosed 0-24 months after a negative screening exam or 6-24 months after a positive baseline screening with a negative recall assessment. PPV-1, sensitivity and specificity were defined as described in figure 3.2.

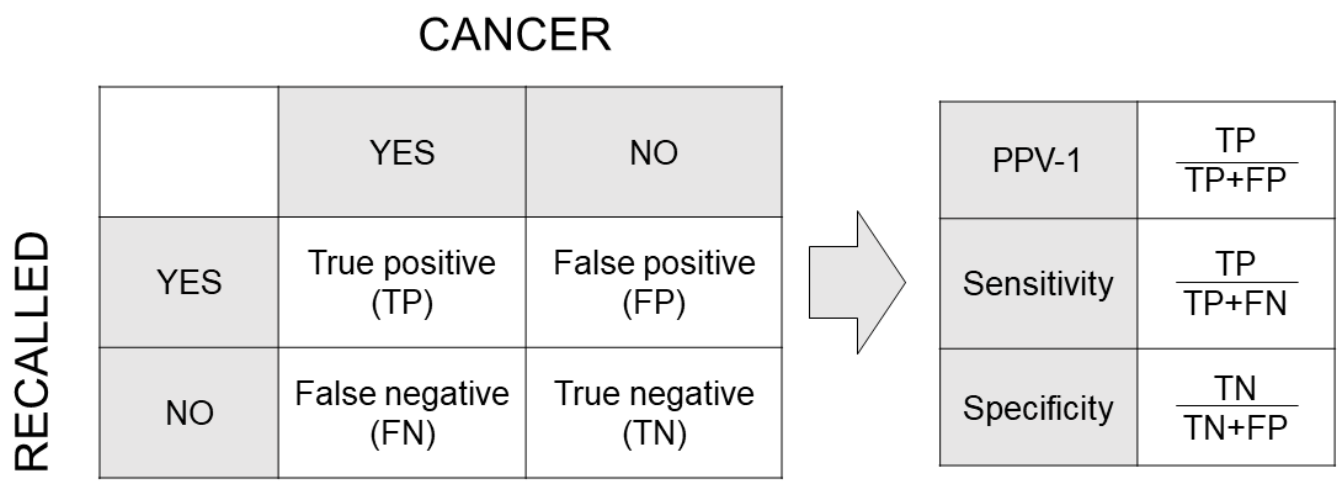

Figure 3.2. Definitions of true positive, false positive, false negative and true negative screening examinations, positive predictive value of recall (PPV-1), sensitivity and specificity of the screening examinations.

In this study we included analyses on histopathological tumor type and grade, grouped tumor diameter ( $\leq 10 \mathrm{~mm} ;>10 \mathrm{~mm}$ to $\leq 20 \mathrm{~mm} ;>20 \mathrm{~mm}$ ), and lymph node status. We also reported subtypes as described in table 1.1; Ki-67<30\% was considered "low" (22). Data were analyzed by screening technique (DBT+SM or DM), screening history (baseline or consecutive) and detection mode (screen-detected or interval cancer) with descriptive statistics. Age in years was presented as mean with standard deviation (SD). Recall, screendetected cancer, interval cancer and histopathological characteristics were presented as 
frequencies and rates per 1000 women screened, whereas PPV, sensitivity and specificity were presented as percentages.

Due to the descriptive approach, simple statistics were used; differences across the study groups were tested with two-sample t-tests and chi-square tests. We used the Bonferroni correction to adjust for multiple testing, and considered $p<0.001$ as statistically significant. IBM SPSS Statistics version 25 was used for statistical analyses.

\subsection{Study 2 - The review study}

\subsubsection{Ethics and approvals}

The Data Protection Officer for the Cancer Registry of Norway and the Department Heads and/or research administration at the local breast centers approved this retrospective study (approval number 2016/4696). This also included use of de-identified mammograms as illustrations. The main aim of the study was radiological learning and the study was thus considered a quality improvement study within BreastScreen Norway. The Cancer Registry Regulations waived the requirement to obtain written informed consent (107).

\subsubsection{Study methodology}

The review study was a retrospective observational multicenter study. We wanted to include reviewed cases and radiologists from all breast centers in BreastScreen Norway (16 at the time of the study). Further, radiologists who did not take part in the review were invited to observe the reviews for learning and educational purposes. We performed a fully informed, consensus-based review study, with all images and information from screening and recall assessment available to the reviewers. The 16 breast centers were divided into 8 pairs. The consensus panels consisted of 5 breast radiologists: 2 from each breast center in the pair, in addition to the $\mathrm{PhD}$ candidate who participated in all 16 review sessions.

Lists of the cancer cases that were to be reviewed were sent to the breast centers in advance, and the breast centers prepared work lists for review in the picture archiving and communication system (PACS). Prior to each review session, the classification system and general instructions for the review were presented to the panel. All images were reviewed locally from PACS. Hanging protocols were not standardized across centers, as the combinations of mammography equipment, work stations and PACS varied. A representative from the Cancer Registry attended all reviews to register the classifications made by the 
reviewing radiologists. We spent 16 hours (2 working days) on each review session (Table 3.1). The review procedure is described in detail in section 3.2.4.

Table 3.1. Schedule for each review session

Review Schedule

\begin{tabular}{lr}
\hline Presentation of classification systems and review procedure to the & 1 hour \\
reviewers & 7.5 hours \\
Review of 75 screen-detected cancer cases, 6 minutes per case & 7.5 hours \\
Review of 75 interval cancer cases, 6 minutes per case & 16 hours
\end{tabular}

\subsubsection{Study sample}

Eligible cases for review were identified from the Cancer Registry's database, eligibility criteria are listed in table 3.2.

Table 3.2. Eligibility criteria for the radiological review of mammograms from screen-detected and interval cancer.

Eligibility criteria for radiological review

\begin{tabular}{c|c}
\hline Screen-detected cancer & Interval cancer \\
\hline - Diagnosed 2006-2015 & $\begin{array}{l}\text { Diagnosed as interval cancer 2004-2016 } \\
\text { - Screening exam in the screening round } \\
\text { prior to diagnosis }\end{array}$ \\
$\begin{array}{l}\text { Digital mammograms from both prior } \\
\text { screening and diagnosis }\end{array}$ & $\begin{array}{l}\text { Prior screening exam } \leq 24 \text { months prior to } \\
\text { diagnosis }\end{array}$ \\
\hline
\end{tabular}

Samples of at least 75 screen-detected and 75 interval cancer cases from each of the 16 participating breast centers, in total 2400 cases, were extracted from the database of the Cancer Registry of Norway. A total of 150 cases $(75+75)$ was set due to the limit of maximum 16 hours (two working days) at each session for screen-detected and interval cancer in total. At some review sessions, the aim of 150 cases was not reached within this time limit, thus the number of reviewed cases was reduced. Further, some of the low-volume centers were unable to provide 75 interval cancer cases that met the eligibility criteria, and for some cases, diagnostic mammograms were unavailable at review (e.g. cancer diagnosed at a different breast center/private institute from where screening was performed). A lack of available interval cancer cases was at some centers compensated by reviewing more than 75 cases of screen-detected cancer (Table 3.3).

The total study sample reviewed included mammograms from diagnosis and prior screening for 1225 women diagnosed with screen-detected cancer after abnormal findings at screening and 1010 women diagnosed with interval breast cancer. 
Table 3.3. Number of reviewed screen-detected and interval cancer cases at each of the 16 breast centers.

\begin{tabular}{lccc} 
Breast center & $\begin{array}{c}\text { Screen-detected } \\
\text { cancer cases }(\mathrm{n})\end{array}$ & $\begin{array}{c}\text { Interval cancer } \\
\text { cases }(\mathrm{n})\end{array}$ & Total $(\mathrm{n})$ \\
\hline Rogaland & 75 & 75 & 150 \\
Hordaland & 75 & 72 & 147 \\
Oslo & 52 & 57 & 109 \\
Telemark & 76 & 66 & 142 \\
Agder & 75 & 69 & 144 \\
Troms og Finnmark & 75 & 75 & 150 \\
$\varnothing$ stfold & 75 & 66 & 141 \\
Nordland & 77 & 73 & 150 \\
Trøndelag & 75 & 70 & 145 \\
Oppland & 85 & 62 & 147 \\
Møre og Romsdal & 90 & 60 & 150 \\
Sogn og Fjordane & 93 & 54 & 147 \\
Vestfold & 77 & 30 & 107 \\
Hedmark & 74 & 40 & 114 \\
Akershus Øst & 75 & 66 & 141 \\
Vestre Viken & 76 & 75 & 151 \\
\hline Total & 1225 & 1010 & 2235
\end{tabular}

Given these sample sizes, the study had $80 \%$ power to detect a difference in proportions of review classification categories and mammographic findings of about 9 percentage points at a significance level of 0.001 and about 6 at a significance level of 0.05 for an outcome with prevalence $40-55 \%$. Given sample sizes of 400 in both groups, as in subgroup analyses, the corresponding numbers were 15 percentage points at a significance level of 0.001 and about 10 at a significance level of 0.05 .

\subsubsection{Review procedure}

We used the same review procedure for screen-detected and interval cancer. The review procedure and classification categories based on visibility of findings on prior screening mammograms are shown in Figure 3.3 and 3.4, respectively. The mammographic findings at diagnosis and prior screening were classified according to the BI-RADS $5^{\text {th }}$ edition lexicon, as described in Introduction (Figure 1.11). The diameters of the mammographic findings were measured using the electronic calipers of the PACS-workstation. In case of dissent among the review panel, a majority decision was made. In multifocal or bilateral disease, the largest tumor was classified, and if calcifications were seen alongside a mass, asymmetry or distortion, the finding was classified as calcifications only if this was the dominating finding. Information on histopathological tumor characteristics from the Cancer Registry's database was printed on paper and communicated to the reviewers immediately after completion of 
each case, and included histopathological tumor type and grade, tumor diameter, lymph node status and hormone receptor status, as well as Her2-status and Ki-67 index if available.

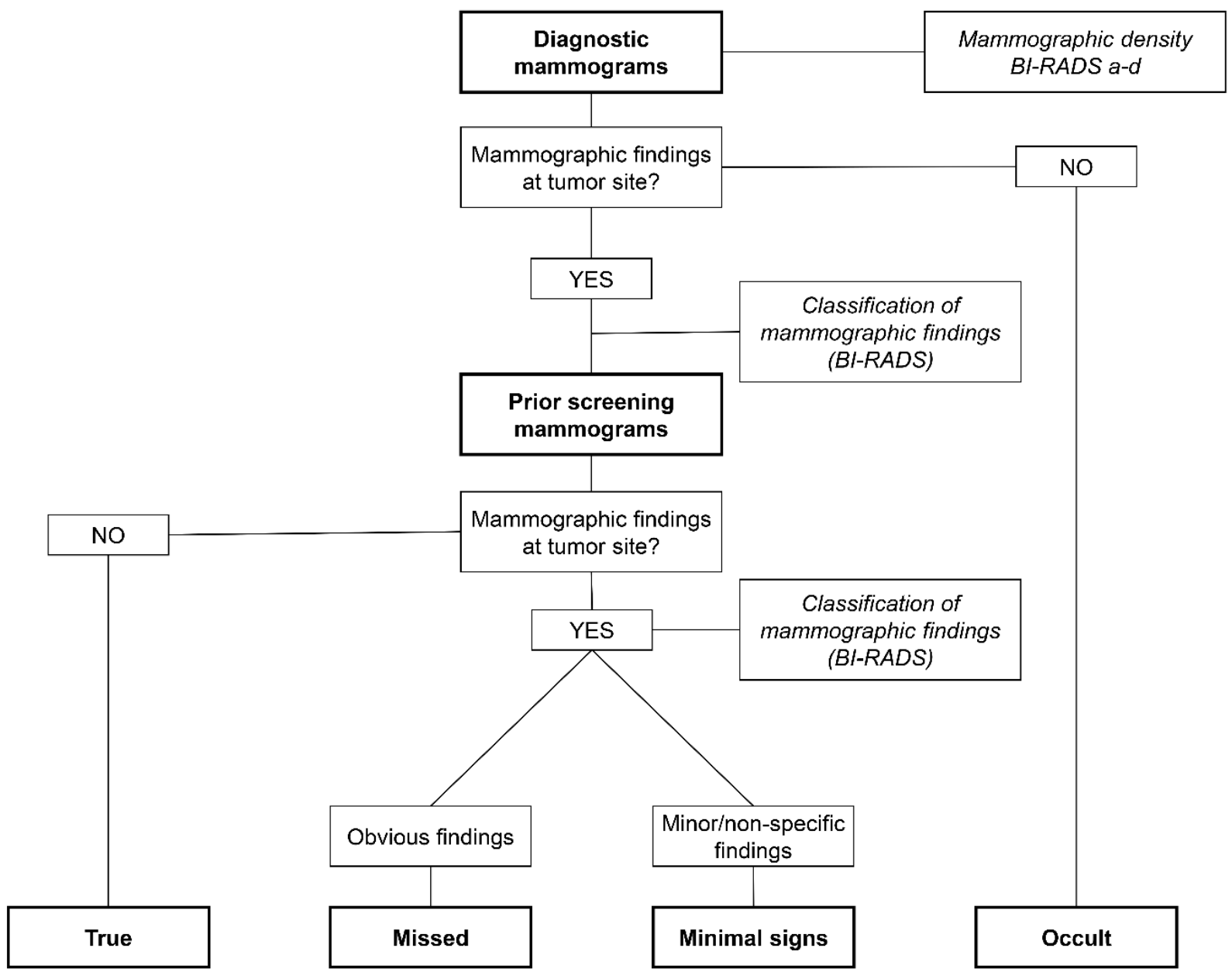

Fig 3.3. Review procedure. Flow chart for radiological review of diagnostic and prior screening mammograms from screen-detected and interval cancer.

Table 3.4. Classification of screen-detected and interval cancer based on findings at prior screening.

Classification category Prior screening mammograms

\begin{tabular}{|c|c|}
\hline True & No visible abnormalities at the eventual cancer site \\
\hline Missed & $\begin{array}{l}\text { Obvious abnormalities visible at the eventual cancer site; should have } \\
\text { resulted in a recall }\end{array}$ \\
\hline Minimal signs actionable* & $\begin{array}{l}\text { Minor visible findings, recall possible but not mandatory within an } \\
\text { accepted recall rate }\end{array}$ \\
\hline $\begin{array}{l}\text { Minimal signs non- } \\
\text { actionable* }\end{array}$ & Non-specific findings at the eventualcancer site; recall not possible \\
\hline Occult & No visible abnormalities at the cancer site at prior screening or diagnosis \\
\hline
\end{tabular}

* Minimal signs actionable and minimal signs non-actionable were merged into one category - minimal signs - for analyses. 
Examples of the different review classification categories are given in Figures 3.4-3.7.
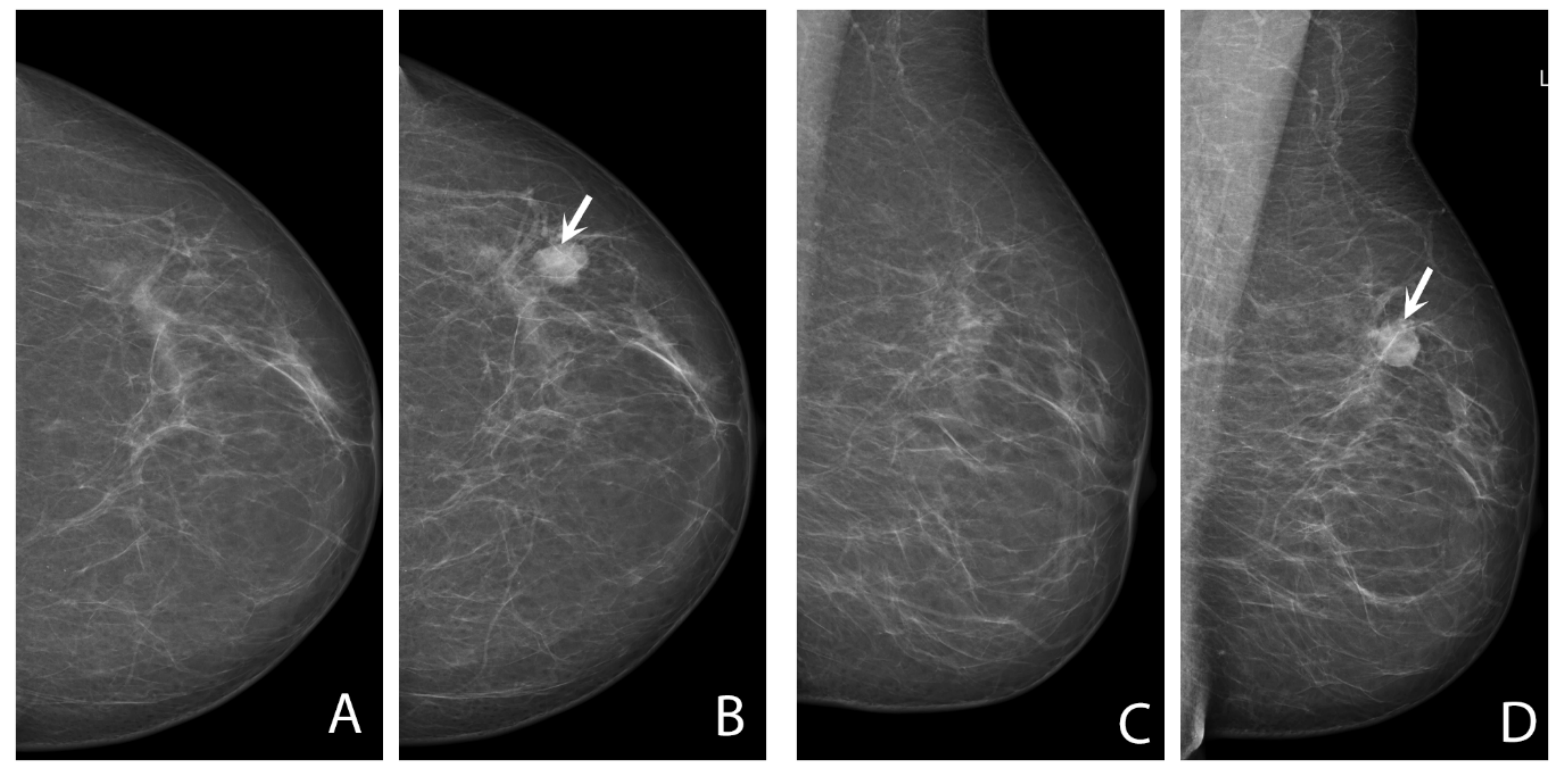

Figure 3.4. True interval cancer: Prior (A) and diagnostic (B) craniocaudal (CC) projections, prior (C) and diagnostic $(D)$ medio-lateral oblique $(M L O)$ projections. No visible abnormalities at the cancer site at prior screening mammograms; a round, obscured mass (arrow) at diagnosis.
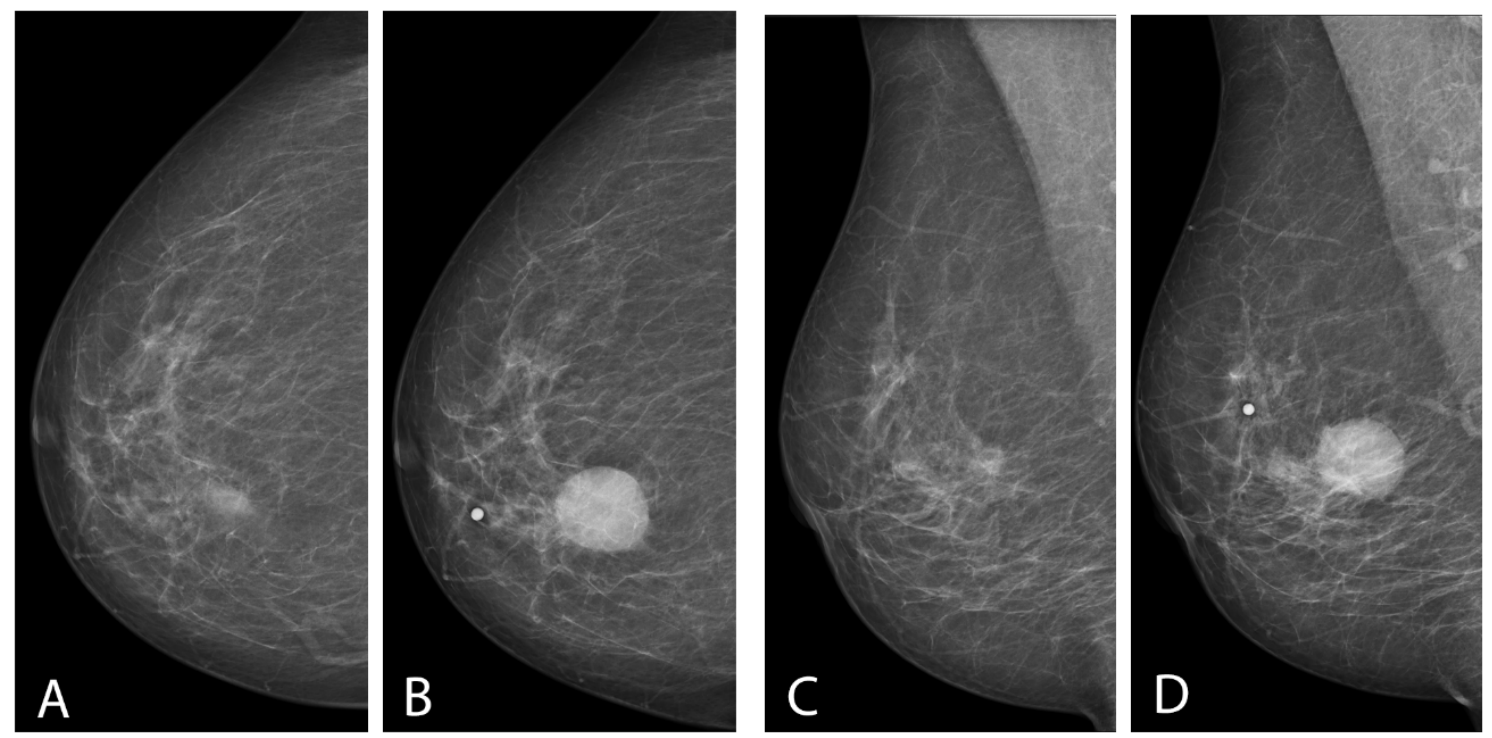

Figure 3.5. Missed interval cancer: Prior (A) and diagnostic (B) CC and prior (C) and diagnostic (D) $M L O$ projections. A focal asymmetry was retrospectively visible at the later cancer site at prior screening, and the cancer presented as a round, obscured mass at diagnosis. 

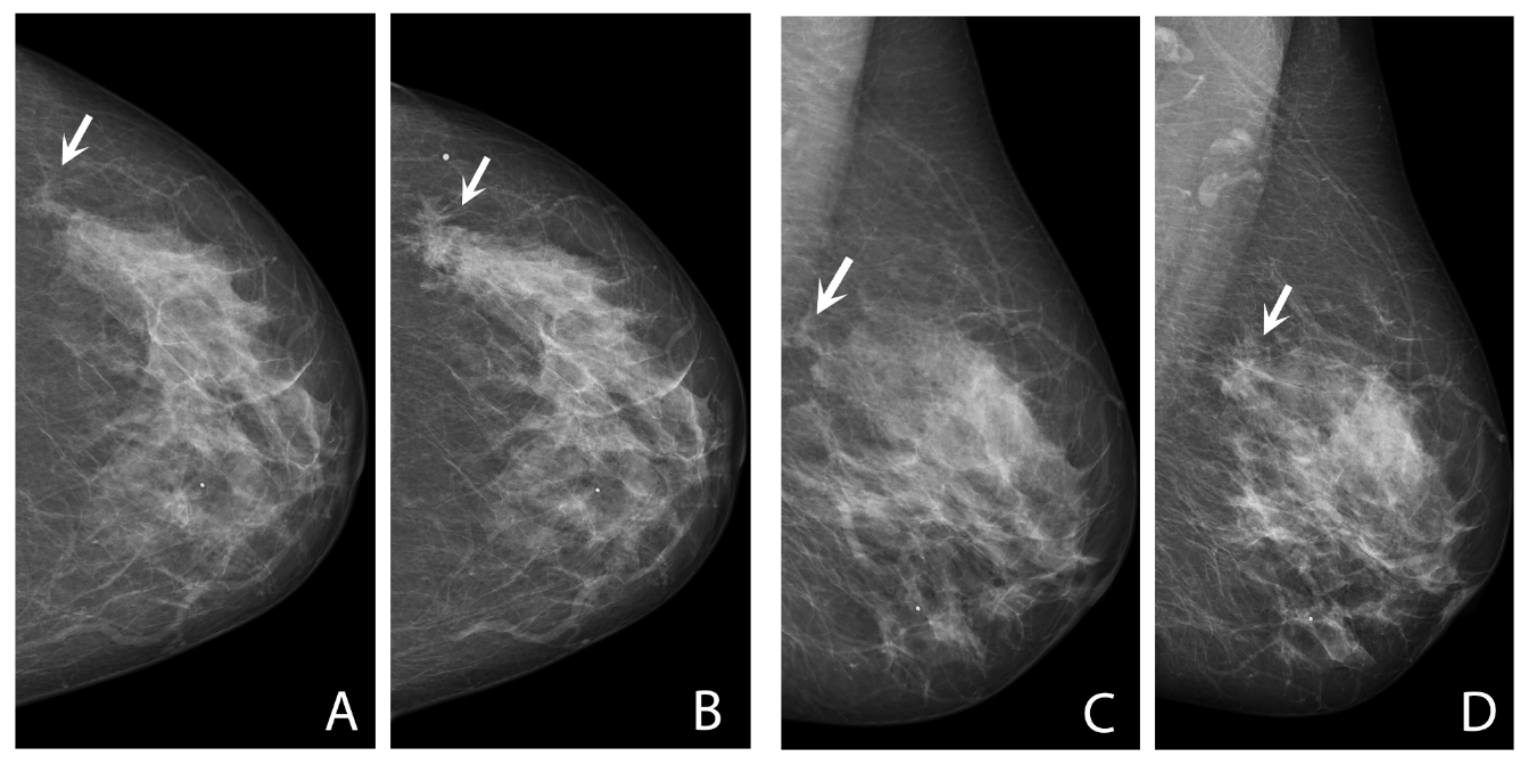

Figure 3.6. Minimal signs interval cancer: Prior (A) and diagnostic (B) CC and prior (C) and diagnostic (D) MLO projections. A focal asymmetry was retrospectively visible on prior screening mammograms at the later cancer site, and the cancer presented as an irregular, spiculated mass at diagnosis (arrows).
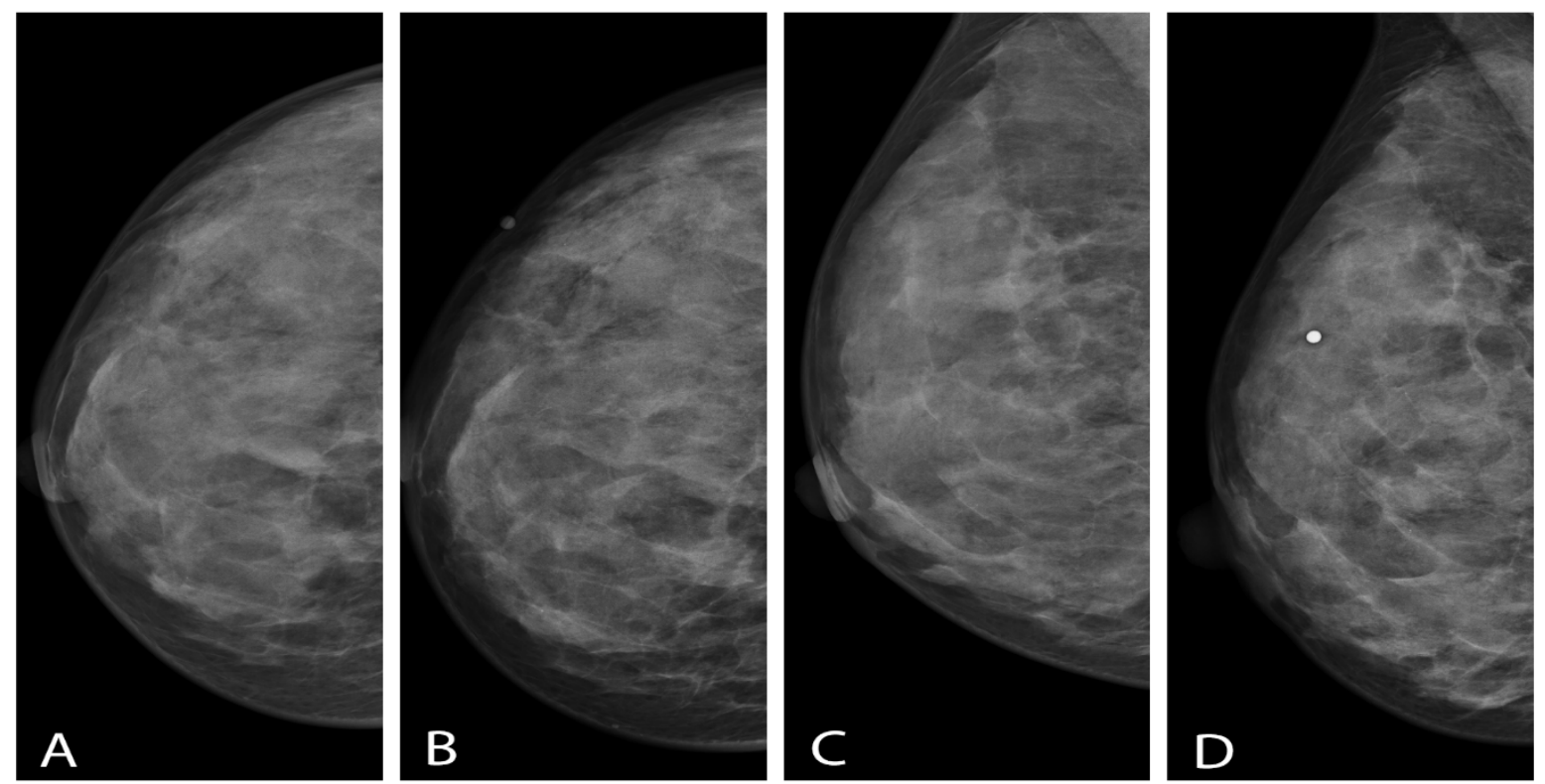

Figure 3.7. Occult interval cancer: Prior (A) and diagnostic (B) CC, prior (C) and diagnostic (D) MLO projections. No visible abnormalities on mammograms at the cancer site, neither at diagnosis nor prior screening. 


\subsubsection{Data analyses}

The analyses were performed on de-identified data received from the Cancer Registry, and included descriptive analyses of age at diagnosis (years) and time from screening to diagnosis in months (for interval cancer). We also described the distribution of review classification categories, mammographic density, mammographic findings at diagnosis and prior screening, and histopathological characteristics.

Categorical data (mammographic findings and histopathological characteristics) were presented as frequencies and percentages. We presented age (years) as mean with standard deviation (SD), and time since screening (months), mammographic and histopathological tumor diameter $(\mathrm{mm})$ as medians with interquartile ranges (IQR). We used Chi-square tests or Fisher's exact test when appropriate (categorical data), independent sample t-tests (means) and the Mann Whitney U non-parametric test (medians) to test for differences between review classification categories and mammographic findings or histopathological characteristics. We used the Bonferroni correction to adjust for multiple testing, and $p<0.001$ was considered statistically significant. IBM SPSS Statistics version 25 was used for all analyses. 


\section{Results/summary of the papers}

\subsection{Study 1 - The OVVV study (paper 1 and paper 2)}

The results from the OVVV-study are presented in two papers; paper 1 is focusing on screening outcome from baseline screening (103) and paper 2 on interval cancer and screening outcome from the consecutive screening round (203). Results from both papers are discussed together in this section, to obtain a coherent whole and a broader perspective.

The recall rate at baseline did not differ between DBT+SM (study group, 33/1000) and DM (control group, 32/1000), $\mathrm{p}=0.41$. The cancer detection rate (DCIS and invasive cancer) was $9.4 / 1000$ in the study group and 6.2/1000 in the control group, $p<0.001$. PPV-1 was thus higher in the study group (28.0\%) than in the control group (19.0\%), $\mathrm{p}<0.001$ (Table 4.1). No differences were observed in interval cancer rate (2.0/1000 in the study group versus $1.5 / 1000$ in the control group, $p=0.12)$, sensitivity of the screening technique $(82.7 \%$ versus $80.1 \%, p=0.35)$ or specificity of the screening technique (97.6\% versus $97.6 \%, p=0.048)$.

In the consecutive screening round two years after baseline, in which all women were screened with DM, the recall rate was lower in the study group (23/1000) compared with the control group (31/1000), $\mathrm{p}<0.001$. The cancer detection rate (DCIS and invasive) was $3.9 / 1000$ in the study group and 5.6/1000 in the control group, $p=0.002$. No difference in PPV-1 was observed (Table 4.1).

Table 4.1. Screening with $D B T+S M$ followed by DM versus DM followed by DM. Unless otherwise specified: data are frequencies with rates pr 1000 screened in parentheses.

\begin{tabular}{|c|c|c|c|c|}
\hline & \multicolumn{2}{|c|}{ Study group } & Control group & \multirow[t]{2}{*}{$p$-value } \\
\hline & $\mathrm{n}$ & pr 1000 & n $\quad$ pr 1000 & \\
\hline Baseline screening & \multicolumn{2}{|c|}{$\mathrm{DBT}+\mathrm{SM}(\mathrm{n}=36641)$} & $\mathrm{DM}(\mathrm{n}=57763)$ & \\
\hline Mean age (years) & 59.1 & (SD 5.8) & $59.4($ SD 5.9) & $<0.001$ \\
\hline Recall & 1158 & (33) & 1873 & 0.41 \\
\hline Screen-detected cancer & 324 & (9.4) & $(6.2)$ & $<0.001$ \\
\hline Interval breast cancer & 68 & $(2.0)$ & (1.5) & 0.12 \\
\hline PPV-1 (\%) & & 28.0 & 19.0 & $<0.001$ \\
\hline Sensitivity (\%) & & 82.7 & 80.1 & 0.35 \\
\hline Specificity (\%) & & 97.6 & 97.4 & 0.048 \\
\hline Consecutive screening & \multicolumn{2}{|c|}{$\mathrm{DM}(\mathrm{n}=26474)$} & DM $(n=45543)$ & \\
\hline Recall & 621 & (23) & 1408 & $<0.001$ \\
\hline Screen-detected cancer & 103 & (3.9) & (5.6) & 0.002 \\
\hline PPV-1 (\%) & & 16.6 & 18.0 & 0.43 \\
\hline
\end{tabular}


At baseline, statistically significantly higher detection rates for DCIS (1.7/1000 versus 0.8/1000), invasive carcinoma NST (6.0/1000 versus 4.3/1000) and invasive tubular carcinoma (0.6/1000 versus $0.03 / 1000)$ were observed for DBT+SM versus DM (Table 4.2). For invasive cancers, the increased cancer detection with DBT+SM compared with DM was mainly due to increased rates of tumors with diameter $\leq 10 \mathrm{~mm}$ (3.1/1000 versus 1.8/1000, $p<0.001)$ and tumors of histological grade 1 (3.0/1000 versus $1.3 / 1000, p<0.001)$. No statistically significant differences were observed in rates of tumors $>20 \mathrm{~mm}$ and grade 2 or 3 tumors between groups (Table 4.2).

Table 4.2. Histopathological tumor characteristics for screen-detected cancer at baseline and consecutive screening for DBT+SM and DM. Mean tumor diameter in mm with standard deviation (SD), otherwise frequencies and rates pr 1000 screened. NA: data not available. DCIS: ductal carcinoma in situ; NST: invasive carcinoma of no special type; ILC: invasive lobular carcinoma.

\begin{tabular}{|c|c|c|c|c|c|c|c|c|c|}
\hline & \multicolumn{4}{|c|}{ Baseline } & & \multicolumn{4}{|c|}{ Subsequent round } \\
\hline & \multicolumn{2}{|c|}{$\begin{array}{c}\text { Study group } \\
\text { DBT+SM } \\
n=324\end{array}$} & \multicolumn{2}{|c|}{$\begin{array}{c}\text { Control group } \\
\text { DM } \\
n=355 \\
\end{array}$} & & \multicolumn{2}{|c|}{$\begin{array}{c}\text { Study group } \\
\text { DM } \\
n=103 \\
\end{array}$} & \multicolumn{2}{|c|}{$\begin{array}{c}\text { Control group } \\
\text { DM } \\
n=254\end{array}$} \\
\hline & \multicolumn{2}{|c|}{$\mathrm{n}$ pr 1000} & \multicolumn{3}{|c|}{$\mathrm{n}$ pr 1000} & \multicolumn{2}{|c|}{$\mathrm{n} \quad \mathrm{pr} 1000$} & \multicolumn{2}{|c|}{$\mathrm{n}$ pr 1000} \\
\hline \multicolumn{10}{|l|}{ Histological type } \\
\hline DCIS & 60 & $(1.7)$ & 45 & $(0.8)$ & * & 19 & $(0.7)$ & 51 & $(1.1)$ \\
\hline NST & 207 & $(6.0)$ & 247 & (4.3) & * & 69 & (2.6) & 157 & (3.4) \\
\hline ILC & 31 & $(0.9)$ & 37 & $(0.6)$ & & 9 & $(0.3)$ & 25 & $(0.6)$ \\
\hline Tubular & 20 & $(0.6)$ & 2 & $(0.03)$ & * & 3 & $(0.1)$ & 5 & $(0.1)$ \\
\hline Other invasive & 6 & $(0.2)$ & 24 & $(0.4)$ & & 3 & $(0.1)$ & 16 & $(0.4)$ \\
\hline Invasive cancer & \multicolumn{2}{|c|}{$\mathrm{n}=264$} & \multicolumn{2}{|c|}{$n=310$} & & \multicolumn{2}{|c|}{$\mathrm{n}=84$} & \multicolumn{2}{|c|}{$n=203$} \\
\hline \multicolumn{10}{|l|}{ Tumor diameter } \\
\hline Mean mm (SD) & 14.0 & $(10.7)$ & 15.9 & (12.1) & & 15.4 & (13.0) & 14.3 & ( 8.2) \\
\hline$\leq 10 \mathrm{~mm}$ & 107 & (3.1) & 103 & (1.8) & * & 31 & $(1.2)$ & 74 & (1.6) \\
\hline $10-20 \mathrm{~mm}$ & 115 & (3.3) & 146 & (2.5) & & 38 & (1.4) & 90 & (2.0) \\
\hline$>20 \mathrm{~mm}$ & 39 & (1.1) & 59 & (1.0) & & 12 & $(0.5)$ & 35 & $(0.8)$ \\
\hline NA & 3 & & 2 & & & 3 & & 4 & \\
\hline Lymph node positive & 50 & $(1.4)$ & 53 & $(0.9)$ & & 16 & $(0.6)$ & 30 & $(0.7)$ \\
\hline NA & 6 & & 5 & & & 1 & & 4 & \\
\hline \multicolumn{10}{|l|}{ Histologic grade } \\
\hline Grade 1 & 105 & (3.0) & 73 & (1.3) & * & 13 & $(0.5)$ & 58 & $(1.3)^{\dagger}$ \\
\hline Grade 2 & 125 & (3.6) & 166 & (2.9) & & 52 & $(2.0)$ & 115 & (2.5) \\
\hline Grade 3 & 34 & $(1.0)$ & 71 & $(1.2)$ & & 19 & $(0.7)$ & 30 & $(0.7)$ \\
\hline NA & 0 & & 0 & & & 0 & & 0 & \\
\hline \multicolumn{10}{|l|}{ Subtype } \\
\hline Luminal A & 147 & $(4.2)$ & 185 & $(3.2)$ & & 35 & (1.3) & 124 & $(2.7)^{\dagger}$ \\
\hline Luminal B Her2- & 93 & (2.7) & 85 & (1.5) & * & 35 & (1.3) & 48 & $(1.1)$ \\
\hline Luminal B Her2+ & 10 & $(0.3)$ & 20 & (0.3) & & 2 & $(0.1)$ & 15 & (0.3) \\
\hline Her2 positive & 2 & $(0.06)$ & 7 & $(0.1)$ & & 5 & $(0.2)$ & 4 & $(0.1)$ \\
\hline Triple negative & 8 & $(0.2)$ & 7 & $(0.1)$ & & 3 & $(0.1)$ & 10 & $(0.2)$ \\
\hline NA & 4 & & 1 & & & 4 & & 2 & \\
\hline
\end{tabular}

* $p<0.001$ compared with study group (baseline)

${ }^{\dagger} p \leq 0.001$ compared with study group (subsequent) 
In the consecutive screening round, the rate of histological grade 1 tumors was $0.5 / 1000$ in the study and $1.3 / 1000$ in the control group $(p<0.001)$ and the rate of Luminal A molecular subtype was $1.3 / 1000$ versus $2.7 / 1000(p=0.001)$. No other differences were observed between the groups at consecutive screening.

Within-group comparison of histopathological characteristics demonstrated lower rates of DCIS, invasive carcinoma NST, tumor diameter $\leq 20 \mathrm{~mm}$, histological grade 1 or 2 and Luminal A/Luminal $B$ negative subtype in the study group at consecutive screening versus baseline (DM after DBT+SM), $p<0.001$ for all. No differences in rates or the distribution of histopathological characteristics were observed for consecutive screening versus baseline in the control group (DM after DM).

The histopathological characteristics for interval cancer did not differ between women screened with DBT+SM and women screened with DM (data not shown). Interval cancers had statistically significantly less favorable histopathological tumor characteristics compared with screen-detected cancers (Table 4.3).

Table 4.3. Histopathological tumor characteristics (frequencies and percentages) for all screendetected and all interval cancers in the OVVV-study. P-values given for the distributions. DCIS: ductal carcinoma in situ; NST: invasive carcinoma of no special type; ILC: invasive lobular carcinoma.

\begin{tabular}{|c|c|c|c|c|c|}
\hline & \multicolumn{2}{|c|}{$\begin{array}{c}\text { Screen-detected } \\
\text { cancer } \\
\mathrm{n}=1036 \\
\end{array}$} & \multicolumn{2}{|c|}{$\begin{array}{l}\text { Interval cancer } \\
n=156\end{array}$} & \multirow[b]{2}{*}{$\mathrm{p}$-value } \\
\hline & $\mathrm{n}$ & $\%$ & $\mathrm{n}$ & $\%$ & \\
\hline Histological type & & & & & 0.007 \\
\hline DCIS & 175 & (17) & 10 & (6) & \\
\hline NST & 680 & (66) & 117 & $(75)$ & \\
\hline ILC & 102 & (10) & 16 & (10) & \\
\hline Tubular & 30 & (3) & 2 & (1) & \\
\hline Other invasive & 49 & (5) & 11 & (7) & \\
\hline Invasive & \multicolumn{2}{|c|}{$n=861$} & \multicolumn{2}{|c|}{$n=146$} & \\
\hline \multicolumn{3}{|l|}{ Tumor diameter } & & & $<0.001$ \\
\hline$\leq 10 \mathrm{~mm}$ & 315 & (37) & 25 & (18) & \\
\hline$>10$ to $\geq 20 \mathrm{~mm}$ & 389 & $(46)$ & 56 & $(41)$ & \\
\hline$>20 \mathrm{~mm}$ & 145 & (17) & 56 & (41) & \\
\hline \multicolumn{3}{|l|}{ Histologic grade } & & & $<0.001$ \\
\hline Grade 1 & 249 & (29) & 23 & (16) & \\
\hline Grade 2 & 458 & (53) & 68 & (47) & \\
\hline Grade 3 & 154 & (18) & 54 & (37) & \\
\hline \multicolumn{3}{|l|}{ Molecular subtype } & & & $<0.001$ \\
\hline Luminal A & 491 & (58) & 61 & $(43)$ & \\
\hline Luminal B Her2 - & 261 & (31) & 43 & (30) & \\
\hline Luminal B Her2 + & 47 & (6) & 13 & (9) & \\
\hline Her2 positive & 18 & (2) & 5 & (4) & \\
\hline Triple negative & 33 & (4) & 21 & (15) & \\
\hline
\end{tabular}




\subsection{Study 2 - The review study (paper 3 and paper 4)}

The results from the review study are presented in two separate papers; paper 3 is presenting results from the review of the screen-detected cancers (204) and paper 4 results from the review of interval cancers (205). In this section, results from the review are discussed as a whole, independently from the papers, to visualize a broader perspective.

\subsubsection{Classification categories and time since screening}

The radiological review of screening and diagnostic mammograms from 1225 screendetected cancers resulted in $46 \%$ classified as true, $22 \%$ as missed and $32 \%$ as minimal signs, among which $16 \%$ were classified as actionable and $16 \%$ as non-actionable. Among the 1010 reviewed interval cancer cases, 35\% were classified as true, 24\% as missed, 28\% as minimal signs ( $15 \%$ actionable and $13 \%$ non-actionable) and $13 \%$ as occult cancers (Table 4.4). In the further analyses, minimal signs actionable and non-actionable were combined into one group, minimal signs.

Table 4.4. Age (years and standard deviation SD), review classification category and mammographic density (frequencies and percentages) for screen-detected and interval cancer. P-values are given for age, and for the distribution of classification categories and mammographic density categories.

\begin{tabular}{lccccr} 
& $\begin{array}{c}\text { Screen-detected cancer } \\
(\mathrm{n}=1225)\end{array}$ & $\begin{array}{c}\text { Interval cancer } \\
(\mathrm{n}=1010)\end{array}$ & \\
\hline & $\mathrm{n}$ & $\%$ & $\mathrm{n}$ & $\%$ & $\mathrm{p}$-value \\
\hline Mean age (years, SD) & 62 & 5 & 61 & 6 & $<0.001$ \\
Review classification category & & & & & $<0.001^{*}$ \\
$\quad$ True & 567 & $(46)$ & 353 & $(35)$ & \\
$\quad$ Missed & 266 & $(22)$ & 246 & $(24)$ & \\
Minimal signs actionable & 192 & $(16)$ & 152 & $(15)$ & \\
Minimal signs non-actionable & 200 & $(16)$ & 133 & $(13)$ & \\
Occult & $\ldots$ & $\ldots$ & 126 & $(13)$ & \\
Mammographic density & & & & & $<0.001$ \\
BI-RADS a & 180 & $(15)$ & 76 & $(8)$ & \\
BI-RADS b & 630 & $(51)$ & 418 & $(41)$ & \\
BI-RADS c & 352 & $(29)$ & 420 & $(42)$ & \\
BI-RADS d & 63 & $(5)$ & 96 & $(9)$ & \\
\hline
\end{tabular}

*Fisher's exact test for the total distribution of classification categories.

Median time from screening to diagnosis for interval cancers was 15 months (IQR 10-20). True interval cancers were diagnosed statistically significantly later in the inter-screening interval than the other categories (Table 4.5). The proportion of cancers diagnosed within the first 12 months after screening ranged from $24 \%$ (true) to $52 \%$ (occult), $p<0.001$. 
Table 4.5 Median time in months with an interquartile range (IQR) from screening to diagnosis, by review classification category for interval cancers. Frequencies and percentages diagnosed within the 0-12 and 13-24 months after prior screening by classification categories.

\begin{tabular}{llrrrr} 
& \multicolumn{1}{c}{ Total } & \multicolumn{1}{c}{ True } & \multicolumn{1}{c}{ Missed } & Minimal signs & \multicolumn{1}{c}{ Occult } \\
\hline Median (months, IQR) & $15(10-20)$ & $17(10-21)$ & $13(4-19)^{\star}$ & $14(10-19)^{\star}$ & $12(8-17)^{\star}$ \\
0-12 months & $369(37 \%)$ & $83(24 \%)$ & $111(45 \%)$ & $109(39 \%)$ & $66(52 \%)$ \\
$13-24$ months & $639(63 \%)$ & $270(77 \%)$ & $135(55 \%)$ & $174(61 \%)$ & $60(48 \%)$ \\
\hline
\end{tabular}

${ }^{*} \mathrm{p}<0.001$ compared with true

\subsubsection{Mammographic density}

Higher mammographic density was observed in interval cancers compared with screendetected cancers (Table 4.4). Among interval cancers, 51\% were classified as BI-RADS c or $d$, compared with $34 \%$ of the screen-detected cancers $(p<0.001)$.

No differences in mammographic density were observed between the categories true, missed and minimal signs screen-detected cancer or between true, missed or minimal signs interval cancer. However, higher mammographic density was observed for occult interval cancer compared with the other interval cancers ( $p<0.001$, Figure 4.1).

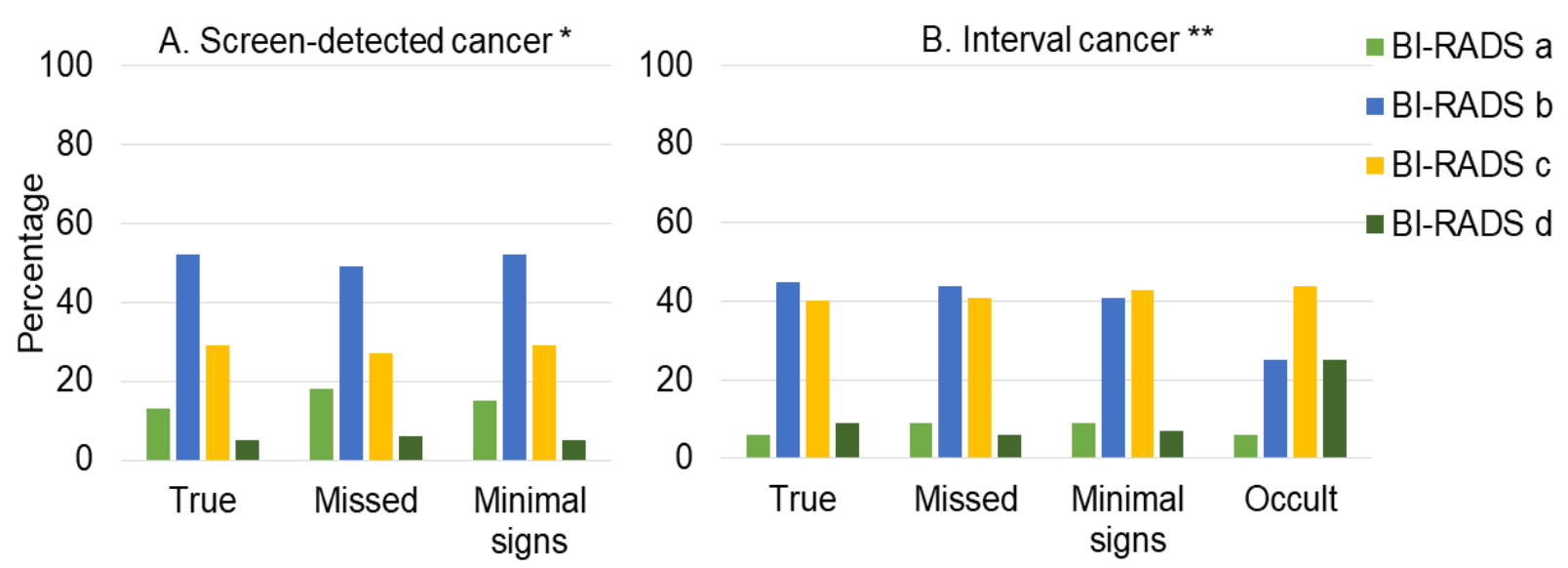

Figure 4.1. Distribution of mammographic density in percentage for each review classification category (true, missed, minimal signs, occult) for screen-detected $(A)$ and interval cancer (B). ${ }^{*} p=0.64$ for the distribution of mammographic density between true, missed and minimal signs screen-detected cancer. ${ }^{*} p<0.001$ for the distribution of mammographic density between true, missed, minimal signs and occult interval cancer ( $p=0.38$ when occult cancer was excluded). 


\subsubsection{Mammographic findings}

The most frequent finding at diagnosis was a mass for both screen-detected (63\%) and interval cancer (57\%). The second most frequent finding was calcifications for screendetected (20\%) and asymmetry for interval cancer (20\%, Table 4.6$)$. The median diameter of mammographic findings at diagnosis was $14 \mathrm{~mm}$ for screen-detected, and $21 \mathrm{~mm}$ for interval cancer, $p<0.001$. The proportion of calcifications was higher in screen-detected than interval cancer at prior screening, otherwise no differences were observed for screen-detected versus interval cancer regarding mammographic findings at prior screening (Table 4.6).

Table 4.6 Mammographic findings at diagnosis and prior screening for screen-detected and interval cancers. Unless otherwise specified, numbers are frequencies with percentages in parentheses.

\begin{tabular}{|c|c|c|c|c|c|}
\hline & \multicolumn{2}{|c|}{ Screen-detected cancer } & \multicolumn{3}{|c|}{ Interval cancer } \\
\hline & $\mathrm{n}$ & $\%$ & $\mathrm{n}$ & $\%$ & \\
\hline \multicolumn{6}{|l|}{ Diagnostic mammograms } \\
\hline Mass & 777 & (63) & 507 & (57) & * \\
\hline Calcifications & 242 & (20) & 6 & (7) & * \\
\hline Asymmetry & 106 & (9) & 179 & (20) & * \\
\hline Distortion & 97 & (8) & 130 & (15) & * \\
\hline Other findings & 3 & $(0.2)$ & 4 & (1) & * \\
\hline Mammographic diameter, median (IQR) & $14 \mathrm{~mm}$ & $(10,21)$ & $21 \mathrm{~mm}$ & $(15,31)$ & * \\
\hline \multicolumn{6}{|l|}{ Prior screening mammograms } \\
\hline Mass & 134 & $(20)$ & 94 & (18) & \\
\hline Calcifications & 142 & (22) & 73 & (14) & * \\
\hline Asymmetry & 302 & (46) & 274 & (52) & \\
\hline Distortion & 78 & (12) & 90 & (17) & \\
\hline Other findings & 2 & $(0.3)$ & & & \\
\hline Mammographic diameter, median (IQR) & $9 \mathrm{~mm}$ & $(6,14)$ & $14 \mathrm{~mm}$ & $(9,20)$ & \\
\hline
\end{tabular}

We observed no differences in the distribution of mammographic findings at diagnosis between true, missed and minimal sign screen-detected cancers. A lower proportion of masses and higher proportion of asymmetries were observed for true versus missed interval cancers ( $p<0.001$ for both, Figure 4.2).

At prior screening, a mass was the most frequent finding in missed screen-detected cancers and the least frequent in minimal signs (43\% versus $5 \%, p<0.001)$. Asymmetry was the second most frequent finding in missed and the most frequent in minimal signs screendetected cancer (25\% versus $60 \%, p<0.001$, Figure 4.2$)$. Asymmetry was the most frequent finding at prior screening for missed and minimal signs interval cancer (36\% and 65\%, respectively, $\mathrm{p}<0.001)$. 

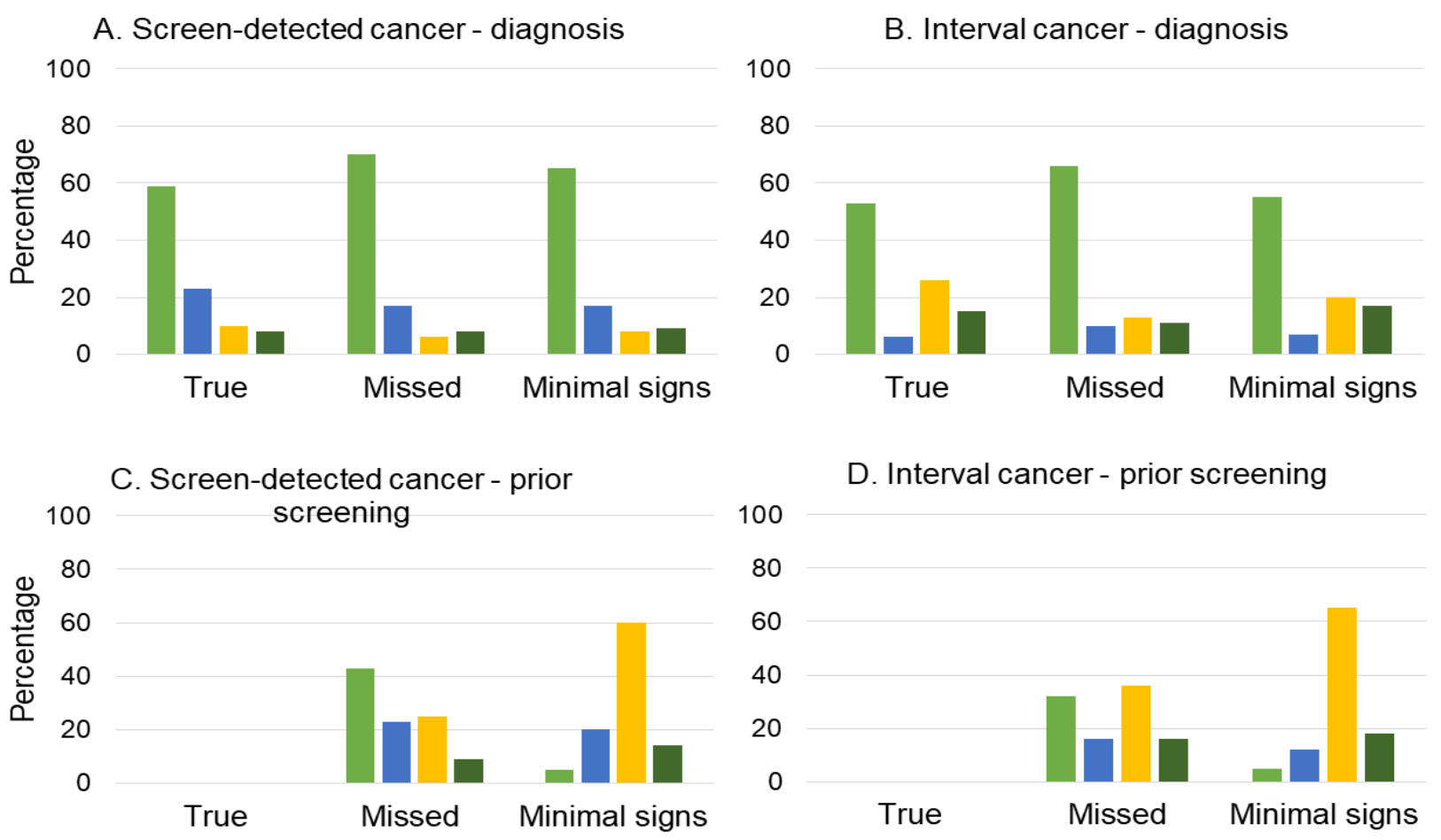

n Mass $\approx$ Calcifications $\backsim$ Asymmetry $=$ Distortion

Mass $\square$ Calcifications $\square$ Asymmetry $\square$ Distortion

Figure 4.2. Mammographic findings in percentages by classification category for screen-detected (A) and interval cancer $(B)$ at diagnosis; screen-detected $(C)$ and interval cancer $(D)$ at prior screening.

Regarding the subclassification of mammographic findings, few differences were observed between screen-detected and interval cancers. In both screen-detected and interval cancers the majority of masses were irregular and indistinct/spiculated, and the majority of asymmetries were one-plane asymmetries (Table 4.7).

Table 4.7. Subclassification of mammographic findings at prior screening for screen-detected and interval cancer; frequencies and percentages.

\begin{tabular}{|c|c|c|c|c|c|}
\hline & \multicolumn{2}{|c|}{ Screen-detected cancer } & \multicolumn{2}{|c|}{ Interval cancer } & \multirow[b]{2}{*}{$p$-value } \\
\hline & $\mathrm{n}$ & $\%$ & $\mathrm{n}$ & $\%$ & \\
\hline Round/oval mass & 60 & $(45)$ & 38 & $(42)$ & 0.71 \\
\hline Irregular mass & 74 & $(55)$ & 52 & $(58)$ & \\
\hline Circumscribed/obscured/microlobulated mass & 7 & $(5)$ & 14 & $(16)$ & 0.009 \\
\hline Indistinct/spiculated mass & 127 & $(95)$ & 75 & $(84)$ & \\
\hline Amorphous/Coarse heterogenous calficications & 34 & (31) & 15 & $(24)$ & 0.28 \\
\hline Fine plemorphic/linear/branching calcifications & 74 & (69) & 48 & $(76)$ & \\
\hline Diffuse/regional calcifications & 15 & (14) & 20 & (31) & 0.02 \\
\hline Grouped calcifications & 68 & (62) & 31 & (48) & \\
\hline Linear/segmental calcifications & 26 & (24) & 13 & $(20)$ & \\
\hline Asymmetry & 136 & (45) & 113 & (41) & 0.59 \\
\hline Focal/global asymmetry & 66 & (22) & 59 & (22) & \\
\hline Developing asymmetry & 101 & (33) & 102 & $(37)$ & \\
\hline
\end{tabular}




\subsubsection{Histopathological characteristics}

More favorable histopathological tumor characteristics were observed in screen-detected cancers compared with interval cancers overall; higher proportions of DCIS, histological grade 1 tumors, lymph node negative and hormone receptor positive tumors and smaller median tumor diameter were observed among screen-detected cancers compared with interval cancers ( $p<0.001$ for all, Table 4.8).

Table 4.8. Histopathological tumor characteristics for screen-detected versus interval cancer. Tumor diameter in $\mathrm{mm}$ with inter-quartile range (IQR), otherwise frequencies and percentages. DCIS: ductal carcinoma in situ; NST: invasive carcinoma of no special type; ILC: invasive lobular carcinoma.

\begin{tabular}{lrrrr} 
& $\begin{array}{r}\text { Screen-deted cancer } \\
(\mathrm{n}=1225)\end{array}$ & \multicolumn{2}{c}{$\begin{array}{r}\text { Interval cancer } \\
(\mathrm{n}=1010)\end{array}$} \\
\hline Histopathological tumor type & $\mathrm{n}$ & $\%$ & $\mathrm{n}$ & $\%$ \\
$\quad$ DCIS & 180 & $(15)$ & 49 & $(5)$ * \\
NST & 880 & $(72)$ & 754 & $(75)$ \\
ILC & 94 & $(8)$ & 129 & $(13)$ * \\
$\quad$ Other invasive & 71 & $(6)$ & 78 & $(8)$ \\
\hline Invasive cancer & & $\mathrm{n}=1045$ & & $\mathrm{n}=961$ \\
\hline Tumor diameter, median (IQR) & $13 \mathrm{~mm}$ & $(9-19)$ & & $(13-25) *$ \\
Histological grade & & & 128 & $(14)$ * \\
$\quad$ Grade 1 & 288 & $(28)$ & 437 & $(47)$ * \\
$\quad$ Grade 2 & 519 & $(50)$ & 364 & $(39)$ * \\
$\quad$ Grade 3 & 222 & $(22)$ & 543 & $(59)$ * \\
Lymph node negative & 797 & $(80)$ & 745 & $(79)$ * \\
Estrogen receptor positive & 919 & $(91)$ & 542 & $(58)$ * \\
Progesterone receptor positive & 730 & $(73)$ & &
\end{tabular}

We did not observe any statistically significant differences in histopathological tumor type or diameter between true, missed and minimal signs screen-detected cancer at diagnosis. A higher proportion of histological grade 3 tumors was observed in true versus missed/minimal signs $(p<0.001)$, and a lower proportion of estrogen receptor negative tumors was observed for true versus missed invasive screen-detected cancer $(p<0.001)$, otherwise no differences were observed between categories for screen-detected cancer (table 4.9).

No differences in histopathological characteristics were observed between true, missed and minimal signs interval cancers. Occult interval cancers were less frequently invasive carcinoma of NST than the other classification categories $(p<0.001)$, and smaller and more frequently histological grade 1 and estrogen receptor positive than true $(p<0.001)$ (Table 4.9). 
No differences were observed between true screen-detected and occult interval cancers ( $p>0.04$ for all characteristics), and no differences in tumor grade or hormone receptor status were observed between true screen-detected and missed/minimal signs interval cancers. The proportion of histological grade 3 tumors was higher in occult interval cancers versus missed/minimal signs screen-detected cancer $(p<0.001)$, otherwise no differences were observed between this groups.

Table 4.9. Histopathological tumor characteristics by review category for screen-detected and interval cancers. Median tumor diameter in $\mathrm{mm}$ with interquartile range (IQR), otherwise data are frequencies and percentages. NA: data not available. DCIS: ductal carcinoma in situ; NST: invasive carcinoma of no special type; ILC: invasive lobular carcinoma.

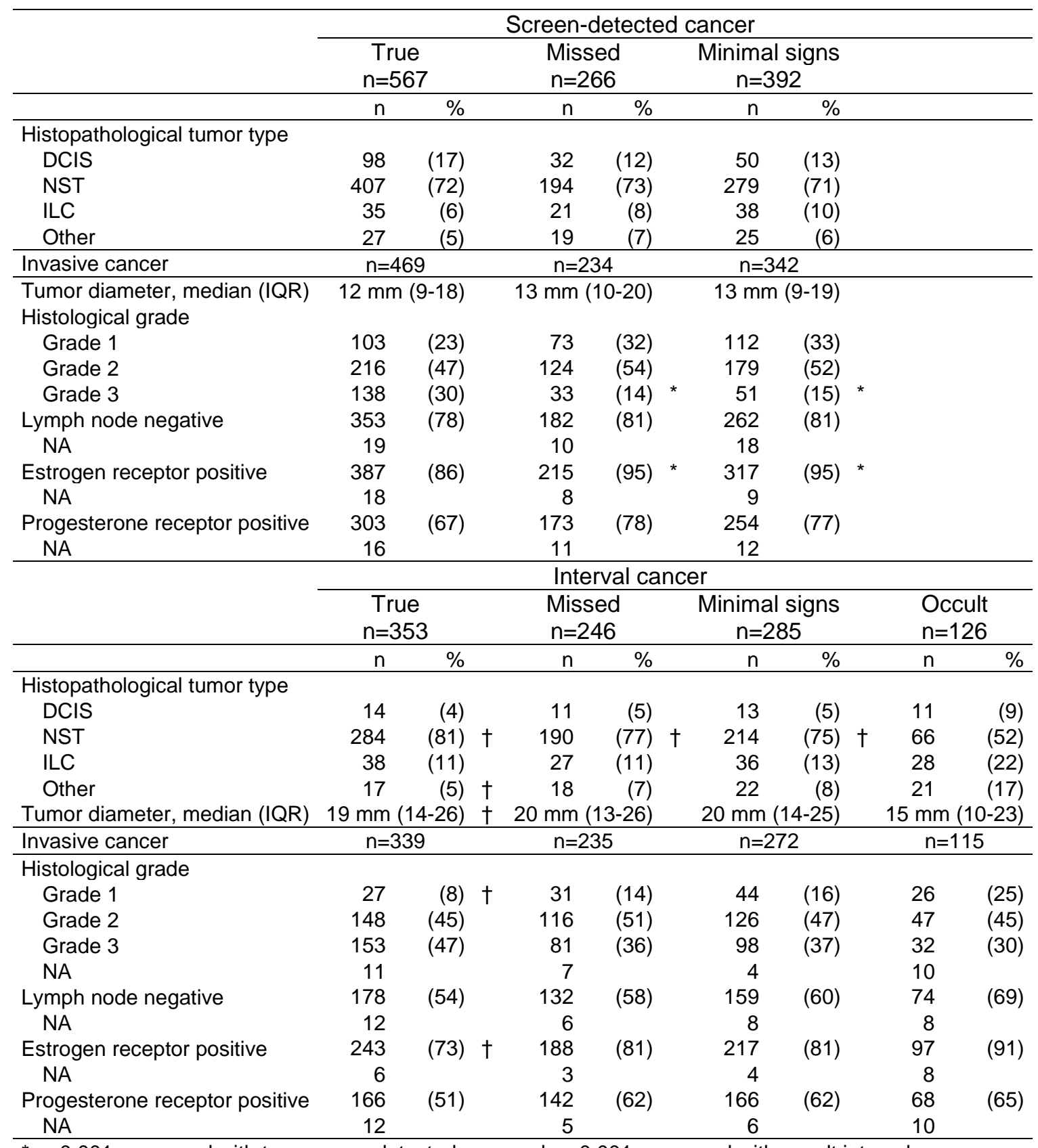

${ }^{*} p<0.001$ compared with true screen-detected cancer. $\dagger p<0.001$ compared with occult interval cancer. 
Invasive cancers presenting as calcifications on prior screening mammograms were more frequently of histological grade 3 than cancers previously presenting as masses, asymmetries and distortions, both for screen-detected and interval cancers (Table 4.10). Further, interval cancers presenting as distortions at prior screening were also more frequently hormone receptor positive than cancers presenting as calcifications $(p<0.001)$.

Table 4.10. Histopathological characteristics by mammographic findings at prior screening for invasive screen-detected and interval cancer, respectively.

\begin{tabular}{|c|c|c|c|c|c|c|c|c|}
\hline & \multicolumn{8}{|c|}{ Screen-detected cancer - prior screening } \\
\hline & \multicolumn{2}{|c|}{ Mass } & \multicolumn{2}{|c|}{ Calcifications } & \multicolumn{2}{|c|}{ Asymmetry } & \multicolumn{2}{|c|}{ Distortion } \\
\hline & $\mathrm{n}$ & $\%$ & $\mathrm{n}$ & $\%$ & $\mathrm{n}$ & $\%$ & $n$ & $\%$ \\
\hline \multicolumn{9}{|l|}{ Histological grade } \\
\hline Grade 1 & 48 & (38) & 14 & (18) & 93 & (32) & 30 & (41) \\
\hline Grade 2 & 63 & (50) & 36 & $(47)$ & 161 & (55) & 41 & (55) \\
\hline Grade 3 & 15 & $(12)$ * & 27 & (35) & 39 & $(13) *$ & 3 & (4) \\
\hline Lymph node negative & 105 & (88) & 58 & (82) & 228 & (80) & 52 & (74) \\
\hline Estrogen receptor positive & 115 & (94) & 71 & (95) & 269 & (95) & 75 & $(100)$ \\
\hline \multirow[t]{4}{*}{ Progesterone receptor positive } & 90 & (74) & 47 & $(68)$ & 225 & $(80)$ & 63 & (85) \\
\hline & \multicolumn{8}{|c|}{ Interval cancer - prior screening } \\
\hline & \multicolumn{2}{|c|}{ Mass } & \multicolumn{2}{|c|}{ Calcifications } & \multicolumn{2}{|c|}{ Asymmetry } & \multicolumn{2}{|c|}{ Distortion } \\
\hline & $\mathrm{n}$ & $\%$ & $n$ & $\%$ & $n$ & $\%$ & $n$ & $\%$ \\
\hline \multicolumn{9}{|l|}{ Histological grade } \\
\hline Grade 1 & 20 & (22) & 4 & (7) & 34 & (13) & 17 & (19) \\
\hline Grade 2 & 42 & (46) & 19 & (33) & 124 & (48) & 57 & (64) \\
\hline Grade 3 & 29 & $(32) *$ & 34 & (60) & 101 & (39) & 15 & (17) \\
\hline Lymph node negative & 58 & (63) & 30 & (52) & 162 & (63) & 41 & (48) \\
\hline Estrogen receptor positive & 75 & (82) & 39 & (68) & 212 & (80) & 79 & (91) \\
\hline Progesterone receptor positive & 59 & (66) & 22 & (39) & 165 & $(63) *$ & * 62 & (71) \\
\hline
\end{tabular}

${ }^{*} \mathrm{p}<0.001$ compared with calcifications 


\section{Discussion}

\subsection{Interpretation of the main findings}

This PhD project was aimed at increasing the knowledge about DBT and about missed and true cancers in organized mammographic screening. We identified a higher rate of screendetected cancer for screening with DBT compared with standard DM and no differences in interval cancer rates between the two screening techniques. Screening with DBT resulted in higher rates of small tumors with low histologic grade compared with standard DM.

The radiological reviews of prior screening and diagnostic mammograms from women diagnosed with screen-detected and interval cancer classified $46-48 \%$ as true or occult, $22-$ $24 \%$ as missed, and $28-32 \%$ as minimal signs. The least favorable histopathological tumor characteristics were observed in interval cancers in general, and in particular true interval cancers, whereas true screen-detected cancers had less favorable characteristics than the other screen-detected cancers. Higher mammographic density was observed in interval cancers compared with screen-detected, and the highest mammographic density was observed in occult interval cancers.

\subsubsection{Screening with DBT}

We found an increased cancer detection with DBT, which was in line with other prospective studies comparing screening with DBT+DM/SM versus standard DM, as shown in Table 1.4 (Introduction). Our study design differed from most of the other studies by using a prospective unpaired design, i.e. the study population included two different, contemporary groups, screened with DBT+SM and DM, respectively. The unpaired design ensured independence between the screening results in the study and control group; the screenreading, consensus and recall assessment was performed separately for women screened with DBT+SM versus standard DM. In contrast, most other prospective non-randomized studies were based on sequential reading of DBT and DM images from the same women $(39,45,60,62,156)$. In the studies from Oslo (OTST) and Malmö (MBTST), four different radiologists performed screen-reading of each exam, followed by a consensus meeting including all images, a design that might potentially have increased the cancer detection as well as reduced the interval cancer rates $(158,165)$. In the STORM studies, sequential reading was performed by the same radiologist, and women were recalled if screen-reading was positive in either step, which may also have biased the results in favor of DBT $(45,62)$. Nevertheless, the results on cancer detection in these studies were comparable to our study. 
Two prospective, unpaired studies from Italy compared screening with DBT+SM with historical cohorts screened with DM. They observed cancer detection rates in accordance with our study; 9.3/1000 (63) and 8.4/1000 (157) for DBT+SM and 5.4/1000 and 5.5/1000 for $\mathrm{DM}$, respectively. The historical cohorts in these studies were screened immediately prior to the DBT-cohort, thus, a potential effect of different timing was probably negligible. Using a contemporary cohort in our study was a strength in that respect.

The gold standard for evaluating the effect of screening with DBT versus DM is randomized controlled trials (RCT). The Tomosynthesis in Bergen (To-Be) 1 trial was an RCT performed within BreastScreen Norway during 2016-17 randomizing more than 28000 women to either screening with DBT+SM or standard DM (64). Unlike the previous non-randomized trials, the detection rate of screen-detected breast cancer in the To-Be 1 did not differ statistically between DBT+SM (6.6/1000) and DM (6.1/1000). In another RCT, The Reggio Emilia Tomosynthesis Randomized Trial (RETomo), interim analyses of half of the study sample demonstrated 90\% increase in cancer detection for DBT+DM (8.6/1000) compared with DM (4.5/1000) (65). However, comparing these two studies is not straightforward. Both studies used mammography equipment from GE, performed double reading with consensus/arbitration and had comparable recall rates, but To-Be included SM while RETomo included DM. However, SM is considered comparable to DM regarding cancer detection, though the vast majority of these studies were performed with equipment from Hologic (43-47). In RETomo, women aged 45-70 were included, and women aged 45-50 were screened annually, the remaining women were screened biennially. In To-Be, women aged 50-69 were included, all screened biennially. Exclusion of prevalently screened women in RETomo might have reduced the detection in both the DBT and the DM arm compared with To-Be, as more cancers in general are detected at prevalent screening than subsequent screening (206). In addition, in the RETomo, 1/5 of the recalled women in the DBT arm were referred for early recall within 9 months after screening, and cancers detected at early recall were included in the calculated rate of screen-detected cancer. Early recall is neither performed in BreastScreen Norway nor in the To-Be trial. Thus, these cancers might be classified as interval cancers in To-Be. Additionally, radiologists' experience, hanging protocols and equipment might have varied between the studies. All these factors may in varying degrees have contributed to the diverging results regarding cancer detection in these studies.

Whether the increased detection with DBT+DM/SM observed in most studies is caused mainly by earlier detection due to increased lead time, or an increased detection of cancers that otherwise not would have been diagnosed (overdiagnosis) is not clear. Both are probably contributing factors. Few studies report results from consecutive screening rounds 
after screening with DBT - however, following the women in the next screening round is one way to understand this issue. In the Verona study (207), half of the women screened in the consecutive round after DBT+SM were screened with $\mathrm{DBT}+\mathrm{SM}$ and half with $\mathrm{DM}$. The cancer detection rate at baseline screening with DBT+SM was $9.3 / 1000$, whereas the cancer detection rates at consecutive screening were 8.1/1000 (DBT+SM) and 3.5/1000 (DM). In a retrospective study from BreastScreen Norway (161), the increased cancer detection sustained for two consecutive screening rounds with DBT+DM/SM (9.9/1000 in the first screening round, and 8.3/1000 in the consecutive round), whereas the cancer detection rate declined to $4.3 / 1000$ for DM for women screened with DM after screening with DBT+DM/SM. These results were in line with our finding of lower cancer detection at consecutive screening for women screened with DM after DBT+SM, even lower than for screening with DM only. This may support the understanding of a prevalent effect with increased lead time for DBTscreening, and further, an element of overdiagnosis cannot be excluded.

To evaluate whether the cancers detected with DBT+SM/DM differed from those detected with DM, the histopathological characteristics at baseline and consecutive screening were analyzed. Our results were in accordance with results from other studies; both the RETomo trial, the OTST and a study from Spain demonstrated higher rates of small and low grade tumors among women screened with $\operatorname{DBT}(60,61,65)$. Further, results from the Verona study (207) demonstrated a higher proportion of stage 1 cancers among women screened with DBT compared with DM. In the MBTST and the To-Be trial, no differences in histopathological characteristics were observed between groups $(39,64)$, and in the Trento study, screening with DBT increased the cancer detection rate across stages, tumor diameter and histological grade (157). Although results from the studies are not unanimous regarding histopathological prognostic and predictive tumor characteristics, one may conclude that tumors detected by DBT might be less aggressive compared with DM.

In the consecutive screening round, we observed slightly less favorable histopathological tumor characteristics in the study group screened compared with baseline screening, whereas no such differences were observed in the control group. This supported our findings of increased early detection of less aggressive cancers with DBT; these were not diagnosed at consecutive screening as they were diagnosed two years earlier by DBT.

At the start of this PhD project, relatively few studies had reported rates of interval cancer for screening with DBT. One reason for this was that studies on interval cancer need sufficient follow-up time (one screening round), and when the OVVV-study started, the studies that had reported results for screening with DBT were largely recent. However, due to increased follow-up time, the number of papers reporting on interval cancer and DBT has since 
increased. Our finding of no statistically significant difference in interval cancer rate between screening with DBT+SM versus DM is in keeping with most other studies $(104,157,159$, $160,162-164)$. One exception is MBTST, in which a statistically significant lower interval cancer rate for women screened with DBT compared with a historical cohort screened with DM was recently reported (165). However, the MBTST was a small study (the number of interval cancer cases was 21 in the DBT group). Further, the interval cancer rate in the DM group (a historical cohort) was markedly higher (2.8/1000) than the interval cancer rate in both the DBT+SM group and the DM group in our study. The interval cancer rates in both groups in our study are consistent with the overall interval cancer rate of 1.8/1000 in BreastScreen Norway during 1996-2014 (3).

We did not observe any differences in histopathological tumor characteristics for interval cancer detected after screening with DBT+SM versus DM. Few other studies report on histopathological tumor characteristics for interval cancer after screening with DBT, and those who do are mainly in line with our study $(104,160,161,163)$.

One major challenge concerning interval cancer and DBT is that interval cancer rates in general are low, which require larger study populations than what is published to reach statistical significance at the observed rates. Due to our large study sample and almost $100 \%$ completeness of the database at the Cancer Registry of Norway on interval cancer, our study has one of the highest number of interval cancers $(n=156)$ among the published studies. However, even larger studies on aggregated data are necessary. Our study has been included in published meta-analyses on interval cancer, but even these meta-analyses proved to be insufficiently powered $(166,167)$. Additional long-term studies on interval cancer rates in consecutive screening rounds may add knowledge.

Our findings regarding interval cancer rates and histopathological characteristics of baseline and consecutive screening cancer may indicate that the excess cancers detected with DBT were not the cancers that otherwise would have been diagnosed as interval cancer. They might rather have been diagnosed as screen-detected cancer in the consecutive screening round, or even not diagnosed at screening at all. Thus, we were not able to demonstrate that screening with DBT has the potential to increase the detection of "killing cancers".

However, early detection and increased detection of breast cancer, even if less aggressive, may add a benefit. Malignant breast tumors do not come with a label which informs whether a particular tumor is potentially lethal for the individual woman. The better prognosis and reduced mortality demonstrated for small and less aggressive tumors result from studies performed at a group-level. For individual women, early diagnosis even of a less aggressive tumor, may be beneficial and life-saving. Additionally, smaller tumors with less aggressive 
characteristics need less extensive treatment, both as regards surgery and systemic treatment $(208,209)$. This may reduce the physical and psychological burden of the disease to women compared with more aggressive disease, and reduce the late adverse effects of the treatment. Further, less extensive treatment can potentially lead to reduced costs for society, both as regards direct expenses related to treatment but also with respect to shortterm and long-term morbidity among the patients.

On the other hand, the increased detection of smaller and less aggressive tumors may potentially increase the burden of overdiagnosis (157), as described in section 1.5.4. Overdiagnosis is usually related to less aggressive tumors including tumors of low histologic grade, tubular carcinomas as well as DCIS, the latter which increased markedly after implementation of mammographic screening $(15,20)$. In our study and in the RETomo study, the detection of DCIS was higher in women screened with DBT, but this is not reported from other studies. However, estimation of overdiagnosis in standard screening as well as in screening with tomosynthesis is challenging, and it is even more challenging or impossible to recognize which tumors are potentially overdiagnosed for the individual women, as overdiagnosis is an epidemiologic term rather than a countable entity. Thus, the rates of screen-detected and interval cancers, as well as tumor characteristics must be used as proxies to evaluate the balance of the possible beneficial and harmful effects of DBT as a screening tool.

\subsubsection{Screen-detected versus interval cancer}

Both the OVVV-study and the review study demonstrated less favorable histopathological tumor characteristics for interval versus screen-detected breast cancer. This was anticipated and well in line with other studies, which have demonstrated that interval cancers are in general larger, of higher histological grade, less frequently DCIS, with less favorable molecular subtypes, and have a higher risk of relapse and less favorable survival than screen-detected cancers $(173,188-196,210-212)$. Further, studies have identified genetic or molecular differences between the screen-detected and interval cancer, though these differences have been less marked or disappeared after adjusting for confounders as age, mammographic density and histologic type and grade $(192,213,214)$. The worse survival for interval cancer persists even after adjustment for histopathological tumor characteristics $(195,196,215,216)$. Thus, to improve detection of "killing cancers" and hence the mortality rates from breast cancer, a focus on reduction of interval cancer rates seems reasonable.

The proportions of true, missed, minimal signs and occult screen-detected and interval cancer vary between studies, but overall our results are fairly in line with other studies (173, $174,210,217,218)$. Roughly, half of the screen-detected and interval cancers demonstrated 
visible findings in prior screening mammograms at a retrospective review, ranging from nonspecific to obvious findings. When findings are visible on prior screening mammograms, an earlier diagnosis is theoretically possible. Interval cancers are mainly diagnosed due to clinical symptoms, and thus, an earlier diagnosis at screening might have been beneficial. For screen-detected cancers, the benefit of even earlier diagnosis may be debatable, as the cancer is still diagnosed in its pre-clinical phase. Whether the cancer would eventually give rise to symptoms in absence of screening is not always clear. Review studies including mammographic and histopathological characteristics are important, to contribute knowledge and experience to this topic.

\subsubsection{True screen-detected and interval cancers}

We observed a statistically higher proportion of histological grade 3 and estrogen receptor negative tumors in true compared with missed and minimal signs screen-detected cancer. Similar, though not statistically significant findings applied to interval cancer. This is consistent with an assumption that true cancers may be more aggressive as they become detectable within less than one screening round of two years (in Norway). Few studies have provided detailed comparisons of histopathological tumor characteristics between the different classification categories, and the results vary among those doing so. Some studies have demonstrated less favorable tumor characteristics in true compared with missed interval cancer, particularly higher proportions of high histologic grade and hormone receptor negative disease $(210,219-221)$, whereas in other studies, true interval cancers are smaller and more often lymph node negative, although more frequently of high grade, than missed $(173,174,217)$. This might be explained by the fact that true and occult cancers were merged into one category in these studies. In our study, combining true and occult cancers would have underestimated the unfavorable tumor characteristics of true cancers, as we observed that occult interval cancers had statistically significantly more favorable tumor characteristics than true.

In true interval cancers, the mammograms were negative at prior screening and the incidence of true cancers is thus a product of screening interval, growth rate and sojourn time (221). Earlier detection within the given screening interval and the given timing of screening examinations is not achievable. To reduce the incidence of true negative cancers, both screen-detected and interval, more frequent screening might be effective. The median time from screening to diagnosis of true negative interval cancer in our study was 17 months (interquartile range 13-21 months), which is in line with other studies $(210,221,222)$. Annual screening or 18 months intervals might catch some of these cancers at screening, before becoming clinically detectable, as illustrated in figure 5.1. However, the possible benefit of 
increased detection of true screen-detected and interval cancers by shortening the screening interval needs to be well balanced against possible harms of more frequent screening, in particular an increase in false positive screening examinations and overdiagnosis, in addition to increased use of resources (223).

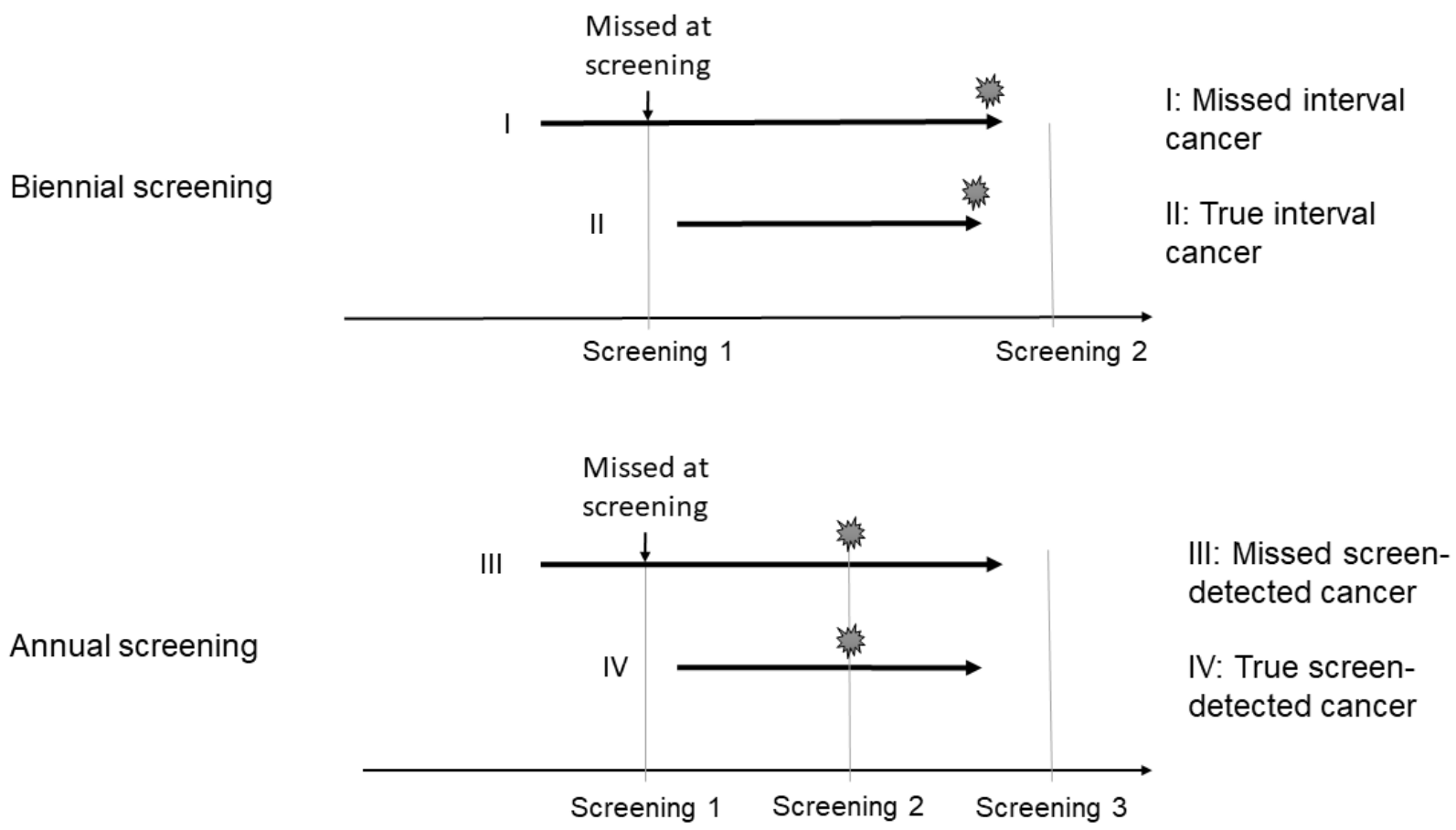

Figure 5.1. The impact of length of screening interval on diagnosis of true and missed cancer. Arrow length represent sojourn time and spiculated lesion time of diagnosis. Tumor I and III have equal sojourn time and are missed at prior screening (screening 1). Tumor II and IV have equal sojourn time and are not visible at prior screening.

\subsubsection{Missed and minimal signs cancer}

In both missed and minimal signs cancers, abnormalities were retrospectively visible on prior screening mammograms, ranging from minor non-specific findings (minimal signs), to obvious suspicious findings (missed cancers). No statistically significant differences were observed regarding the classification into the main categories mass, calcification, asymmetry or distortion between missed and minimal signs cancers at prior screening. With regard to further subclassification of the findings (Figure 1.11), differences applied between missed and minimal signs (204). However, this was an obvious consequence of the design, as allocation into the categories missed or minimal signs was merely based on the suspiciousness of the findings, and the different subclasses mammographic findings have different levels of suspiciousness (86). Thus, analyses of subclassification of mammographic findings in missed versus minimal signs are biased. 
As described earlier, at review, the minimal signs category was further divided into the categories minimal signs actionable and non-actionable. No differences were observed between the two categories regarding the main classification of mammographic findings or histopathological tumor characteristics (data not shown). This, and the fact that both categories presented visible findings at prior screening that were not considered warranting a recall, allowed us to combine the two categories into one, minimal signs.

Missed/minimal signs interval cancers were larger at diagnosis and prior screening compared with missed/minimal signs screen-detected cancer, which may be explained by two factors. The first factor is the timing of the screening exam. If the prior screening exam for those diagnosed with missed or minimal signs interval cancer was scheduled later than the actual screening date, the findings on priors might have been smaller, and the cancer might have been diagnosed as a screen-detected breast cancer at screening two years later rather than interval cancer (Figure 5.2, tumor I and II).

Second, whether a cancer with visible findings at prior screening is detected in an asymptomatic phase at screening, or due to symptoms as interval cancer, is also related to aggressiveness and pattern of growth. This is illustrated by our findings of higher proportions of histological grade 3 , hormone receptor negative and lymph node positive tumors among the interval cancers compared with the screen-detected (Figure 5.2, tumor II and III).

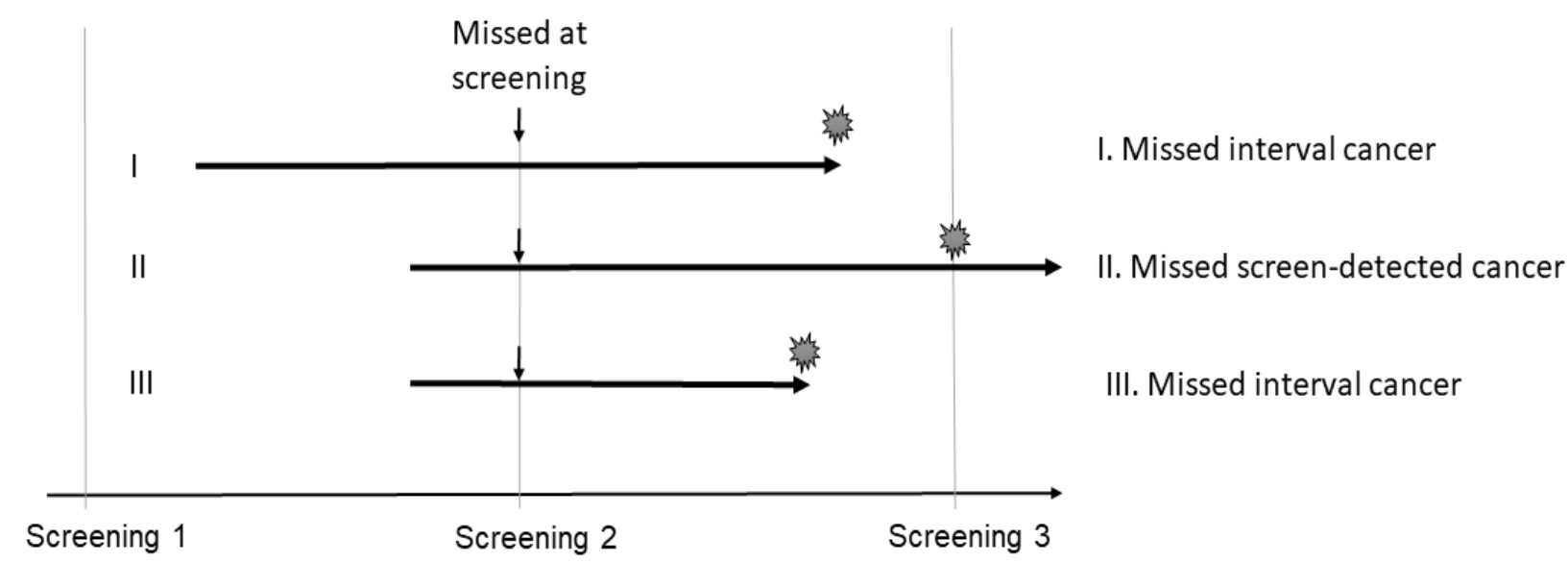

Figure 5.2. Timing of the screening examination and tumor aggressiveness. Arrow length illustrates sojourn time, arrowheads represents the time when tumors become clinically detectable. Spiculated lesions represent time of diagnosis. All cancers are missed or not recalled at first screening (screening 2).

Tumor I and II have similar histopathological tumor characteristics and sojourn time, but different timing of screening examination in relation to start of sojourn time.

Tumor II and III become detectable on mammograms at the same time, but tumor III is more aggressive and with shorter sojourn time than tumor II. 
Except a statistically significantly higher proportion of calcifications in screen-detected cancer, which is probably related to a higher incidence of DCIS, we did not observe any differences in mammographic findings at prior screening between screen-detected and interval cancer in our study. This might indicate a similarity in the mechanisms related to observer error or misinterpretation that leads to a diagnosis of missed/minimal signs screendetected or interval cancer.

Screen readers might identify abnormalities but interpret them as benign and refrain recall for further assessment, leaving the lesions to be diagnosed later as a missed or minimal signs screen-detected or interval cancer. One frequent reason for this is (long-term) lesion stability. The doubling time of a breast tumor has been reported to range from 44 to 1869 days, with an average of 212 days (224). Even if lesion stability over two screening rounds usually indicates a benign lesion, malignant lesions may, from time to time, increase so slowly in size that images from more than two screening rounds are needed to observe lesion growth. Hence, including more than one prior screening exam in screen reading may reduce the proportion of missed cancers. However, it is likely that lesions that remain stable over more screening rounds are less aggressive, slow-growing tumors, for which a delayed diagnosis may not considerably impair prognosis and survival, although it may influence the choice of treatment and thereby possible side-effects of treatment.

Some malignant lesions have a mammographic appearance usually associated with benign lesions, and might be misinterpreted at screening. Such features commonly include circumscribed masses, certain calcifications and asymmetries $(86,96,150)$. The majority of missed and minimal signs cancers in our study were classified as asymmetries at prior screening. One-plane asymmetries are relatively common in screening mammograms and usually represent summation artefacts or superimposition of normal breast tissue (150). Sickles demonstrated in 1998 that $1.8 \%$ of one-plane asymmetries at screening represented malignant lesions (225). Thus, lowering the threshold for recall of such lesions would probably increase recall rates and false positive rates far beyond what is acceptable, which in BreastScreen Norway is 3-5\% (226). A developing asymmetry, however, which is a twoplane asymmetry that is new, larger or more conspicuous than on prior mammograms (86), has a reported likelihood of malignancy of $12.8 \%$ at screening and up to $26.7 \%$ in diagnostic mammograms (227). In our study, a developing asymmetry was observed in $33 \%$ of the missed/minimal signs screen-detected and $37 \%$ of the missed/minimal signs interval cancers at prior screening. Thus, including more than one prior screening exam (150) and a special attention to developing asymmetries in screen-reading seem reasonable to reduce the proportion of missed cancer. The least favorable histopathological characteristics were observed in cancers presenting as calcifications at prior screening; $60 \%$ of the invasive 
interval cancers and $35 \%$ of the screen-detected cancers presenting as calcifications at prior screening were histological grade 3 at diagnosis. The majority of calcifications at prior screening, both in screen-detected and interval cancer, were characterized as fine pleomorphic/linear/branching and grouped or linear/segmental. According to the BI-RADS lexicon, such calcifications are considered category $4 \mathrm{C}$, with a risk of malignancy of more than $50 \%$ but less than $95 \%$ (86). Hence, a failure to recall such calcifications might be related to lack of perception rather than misinterpretation, or due to lesion long-term stability.

Lack of perception of visible radiological abnormalities may be due to several reasons. Inexperience as well as fatigue due to a high work-load, may impair perceptional skills and vigilance $(96,141)$. Several cognitive biases have also been described, including "satisfaction of search", defined as decreased vigilance or awareness of additional abnormalities after identifying the first one (150). Mammograms often present more than one abnormality, and if the screen-reader spend time and effort on one finding, other potentially more suspicious findings may be overlooked. "Inattention blindness" refers to the process of not noticing findings with an unexpected location or appearance $(150,183)$. This is exemplified by lesions in the periphery of the breast or in the axilla, or diffuse abnormalities or asymmetries that may only be visible by taking a broader look at the mammograms.

A recent study by Chang et al demonstrated an association between mammographic appearance and length of sojourn time. For small malignant tumors $(<14 \mathrm{~mm})$ this time was longest for "stellate lesions", shorter for "powdered" and "crushed stone" calcifications and shortest for circular lesions. Further, the risk for interval cancer (the ratio between interval cancer incidence and expected cancer incidence) was lowest for the stellate lesions and highest for circular lesions (94). This adds to the complex decision on which findings to recall or not; stellate lesions roughly correspond to spiculated lesions, which are frequently recalled, whereas circular lesions are non-spiculated lesions including round/oval lesions, which more frequently represent benign lesions and are less frequently recalled.

Studies have demonstrated that double reading in screening increases cancer detection (228). Further, in a Norwegian study, $24 \%$ of cancers were detected by discordant screenreading, i.e. only one of the two screen-reading radiologists recalled the finding (187). Finding the "perfect pair" of radiologists in screen-reading, with perceptive and interpretative skills that complement each other may result in optimization of screening sensitivity. However, this requires a large volume of screening examinations and sufficient data on radiologists' performance, otherwise it might be hardly feasible in an ordinary screening practice. 


\subsubsection{Occult cancer}

Occult cancers are not visible on mammograms, usually due to masking of the lesion by breast tissue, as illustrated by our findings of a higher proportion of BI-RADS density $c$ or $d$ in occult cancers compared with the other categories. However, not all occult cancers were diagnosed in dense breasts: one third were diagnosed in breasts classified with mammographic density a or b. Cancers can also be masked by overlapping tissue in less dense breasts, if the mammographic density of the tumor and the breast tissue are similar. In our study, we also classified cancers as occult if they were located outside the target area of the mammogram. A cancer presenting as Paget's disease of the nipple might also be occult on mammograms.

Occult cancers had the most favorable histopathological characteristics among interval cancers. Additionally, only minor differences in histopathological characteristics were observed between occult interval cancers and the screen-detected cancers. Hence, one may postulate that these cancers were not diagnosed as interval cancers because of intrinsic biological properties, but rather due to extrinsic factors (as dense breast tissue or location) that hamper detection at screening. Further, a study by Holm et al observed more favorable histopathological tumor characteristics of interval cancers detected in breasts with high versus low mammographic density, and the tumor characteristics of interval cancers in dense breasts were comparable with those of screen-detected cancer (185). To be able to detect occult cancers before becoming symptomatic, supplementary screening methods may be applied for women with mammographic dense breasts, as digital breast tomosynthesis, ultrasound or MRI (155).

\subsection{Methodological considerations}

\subsubsection{Data quality}

The Cancer Registry of Norway is responsible for collecting screening data from women who participate in the screening program $(3,107)$. This includes information related to invitations, attendance, and the screening examination including the radiologist's interpretation scores, recall assessment and pathological and surgical reports. Reporting cancer data to the Cancer Registry is mandatory by law, and the registry collects data from among others hospitals, medical and pathological laboratories, hospital discharges and death certificates. The national 11-digit personal identification number assigned to all residents in Norway links screening data with data from the screening database, and data are stored in a national database at the Cancer Registry. This database is almost $100 \%$ 
complete for registration of breast cancer cases in Norway $(3,172)$. Due to this completeness, information on interval cancers is completely available, which was highly important for both the OVVV-study and the review study.

Women with negative screening results have the right to request that their screening data be deleted from the Cancer Registry after the quality of data has been assured, and about 1.9\% of all participating women ever have exercised their right to do so (3). Thus, screening data from these women are not included in quality assurance and research, and not in the studies included in this thesis.

\subsubsection{Missing data}

Missing data was defined as data not available for analyses. Reasons for data to be missing included data not reported or incorrectly reported, not registered or incorrectly registered in the Cancer Registry database (both studies) or at review (the review study), or data excluded during cleaning of the data, either unintentionally or by purpose (exclusion criteria).

In the OVVV-study, no data on histological tumor type were missing, whereas data on tumor diameter, histological grade, lymph node status and molecular subtype were missing in a small number of cases ( $<20$, less than $2 \%$ of cases). The highest number of missing data was seen for tumor diameter and lymph node status, which may be related to neo-adjuvant treatment of the tumors. Pathologists report tumor diameter and lymph node status from surgical specimens, and in women receiving neo-adjuvant treatment, estimation of these measures might be difficult or impossible to report due to treatment response. The increasing frequency of breast cancer patients receiving neo-adjuvant chemotherapy, due to an extension of the indication criteria as described in the Introduction, represents a potential increasing challenge in studies, in particular as the cancers with missing data on tumor characteristics are often those with the least favorable prognosis. This may lead to an underestimation of tumor diameter and aggressiveness. However, pre-treatment data as radiological measures and results from needle biopsies may be used as proxies for the missing data from surgical reports.

In the review study, data on mammographic diameter at diagnosis were missing in 81 screen-detected cancers ( $7 \%$ of the cases). This was mainly related to a systematic lack of data for this variable at one single center $(n=75)$, probably due to error in registration, storage, merging or cleaning of the data. The remaining six were randomly distributed among the other centers, as were also the 31 cases of interval cancer without information on mammographic diameter at diagnosis. Regarding mammographic diameter at prior screening, data were missing in as many as $309(26 \%)$ of the screen-detected and interval 
cancers in total. These data were missing more evenly among the centers (ranging 0-23 cases per center). The major reason for these data to be missing is probably that the lesions were considered unmeasurable at review, and thus, the missing data may have led to an overestimation of the mammographic diameter at prior screening. The majority of the lesions $(n=201)$ were asymmetries, mainly one-plane asymmetries, which may illustrate that these findings were very subtle and difficult to distinguish from surrounding tissue. In retrospect, more effort could probably have been made to perform proper measurement despite minimal abnormalities.

Histopathological tumor diameter was missing in 98 (5\%) of the invasive cancers in the review study, the majority of these were interval cancers. This was probably related to a higher frequency of women receiving neo-adjuvant chemotherapy due to more advanced disease at diagnosis in this group. Data on histological grade, lymph node status and hormonal status were missing in 79, 81 and 72 cases, respectively, the majority in interval cancer. This might have resulted in an underestimation of tumor diameter and aggressiveness.

\subsubsection{Study samples}

The OVVV-study included 92404 screened women at baseline, and based on this, we had $80 \%$ power to observe a difference of 0.2 percentage points or more in both recall and detection rates between SM+DBT and DM at a significance level of 0.05 . We were aware that the study sample was underpowered to demonstrate a statistically significant difference in interval cancer at the observed rates. Post hoc power calculations demonstrated that $>100000$ individuals in each group were needed to obtain $80 \%$ power to detect the observed interval cancer rates at a significance level of 0.05. Lack of statistical power represents a problem in most studies comparing interval cancer rates for screening with DBT and DM.

Our review study is one of the largest review studies performed on screen-detected and interval cancer, in particular with digital mammograms, including 1225 screen-detected and 1010 interval cancers (229). The study had 80\% power to detect a difference in proportions of about 9 percentage points at a significance level of 0.001 and about 6 at a significance level of 0.05 for an outcome with prevalence $40-55 \%$. Given sample sizes of 400 in both groups, the corresponding numbers were 15 percentage points at a significance level of 0.001 and about 10 at a significance level of 0.05 . However, in a radiological review, both the allocation into the different review classification categories as well as classification of mammographic findings will inevitably vary between studies and radiologists, as described in more detail in chapter 5.2.4. Such differences might potentially outweigh the effort of 
increasing sample size in order to obtain sufficient statistical power to identify even smaller differences than this study was powered for.

\subsubsection{Internal validity}

The internal validity of a study refers to the validity of the comparisons or conclusions made within the study population (230). Three main factors challenge the internal validity of a study; selection bias, information bias and confounding bias. Bias is any systematic error that results in an incorrect estimate of the association between exposure and outcome (230)..

\subsubsection{Selection bias}

Selection bias is a systematic error that occurs when differences exist between the exposed and non-exposed groups that distort the association between exposure and effect. Selection bias may result due to the selection procedures, or from factors that influence study participation (230). Participation in BreastScreen Norway is voluntary and women who take part may be more health-conscious than those who do not. Thus, baseline risk of breast cancer and lifestyle factors may differ between screening participants and non-participants, which may cause a selection bias of women studied.

The OVVV-study included women from three different geographical areas; women screened at the breast center in Oslo comprised the study group while women screened at the breast centers in Vestfold and Vestre Viken comprised the control group. Hence, selection bias between groups may have applied, as attending women might have been different in the study and control group. The attendance rate in Oslo is lower than in Vestfold and Vestre Viken (3). Further, sociodemographic factors may differ. Vestfold and Vestre Viken have both urban and more rural population, whereas the population in Oslo is mainly urban. Lifestyle factors and use of hormonal replacement treatment are factors that influence breast cancer risk and mammographic density, and these factors may potentially vary between rural and urban population. Additionally, the proportion of immigrant women was larger in Oslo than the other areas; lower attendance and higher proportions of histological grade 3 and triple negative breast cancer have been demonstrated in immigrant women $(231,232)$. Data on sociodemographic factors, use of hormonal replacement therapy, breast cancer risk, mammographic density and immigration history were not available for analyses and were not included in the estimation. All these factors are mainly drivers in the opposite direction of our results, as we demonstrated higher rates of small tumors of low histological grade in the study group, and may potentially have resulted in underestimation of the increased detection of small, less aggressive tumors. However, the three centers had comparable early performance measures in the pre-study period (103). To reduce the selection bias caused by the three different centers, screening with both DBT+SM and DM could have been performed 
at all three centers. However, this was not feasible, as DBT+SM was not available as screening technique in Vestfold and Vestre Viken.

In analyses from the consecutive screening round, we only included re-attending women. If women invited, but not re-attending, had different characteristics in the study and control group, selection bias might be possible. However, we have no data on this, and consider irregular screening attendance as a part of the program with no reason to assume it was different for women included in the study versus control group (233).

The review study included cases from 16 different breast centers over a time span of 12 years. Hence, the study population was heterogeneous. Further, the number of included cases varied between the centers, as did the reason for not reviewing the intended number of $75+75$ cases at each center. However, as each center only contributed with only $5-7 \%$ of the cases, we do not consider this heterogeneity to bias the results largely in one direction. Further, we did not stratify the analyses by breast center, and did not compare results between the different centers. As all centers in BreastScreen Norway at that time were included in the review and analyses, we consider the cases reviewed as representative for BreastScreen Norway.

\subsubsection{Information bias}

Information bias refers to a systematic error that occurs when the information recorded about the subjects in a study is inaccurate, and can result in misclassification (230). Misclassification is non-differential if it is unrelated to the other study variables and occurs equally in exposed and non-exposed subjects, and differential if the misclassification is not equal between the study groups or vary according to the value of the studied variables (230).

In the OVVV-study, local radiologists performed the screen-reading, consensus and recall assessment, and local pathologists examined the biopsies and surgical specimens. Further, the mammographic equipment, workstations and imaging technique differed across the centers. These factors may have caused information bias between groups, mainly differential misclassification of the outcome variables, as misclassification could have influenced the study and control group differently. However, the three centers had comparable cancer detection rates prior to the study period (3), and we did not observe any differences in cancer detection, PPV-1 or histopathological characteristics in the consecutive screening round in women who were not a part of the OVVV-study population (data not shown in thesis) (203).

The results of the OVVV-study have possibly been affected by a study effect. The radiologists and pathologists participating in the study were aware of the study, and also regularly received preliminary study results, as a part of the usual quality assurance of the 
program (see 1.5.2 in the Introduction). This might have affected the professionals, as they might have been able to adjust their habits regarding screen-reading, recall, assessment and diagnostics. This, in turn, might potentially have influenced the results differently over time during the study period and across centers, either enhancing or diminishing differences.

In the review study, the classification into true, missed, minimal signs and occult cancer, as well as the classification of mammographic findings and mammographic density, was performed by panels of five radiologists. Even if one radiologist (the PhD candidate) participated in all reviews, and instructions on review logistics and classification were given ahead of the reviews to all radiologists, differences in characterization and interpretation across panels and individual radiologists were likely. These differences might have resulted in a non-differential misclassification within each center/panel, as the misclassification most likely was consistent across cases within each panel. Differences in classification across centers/panels might have resulted in a differential misclassification. However, all decisions within the panels were majority decisions and all analyses were performed on data from all reviews in total, not by each center. This might have reduced the magnitude of the bias by averaging out the effects of misclassifications.

Additionally, the combination of mammography equipment, PACS and workstations as well as the hanging protocols, differed across the 16 centers. Further, findings were classified based on the last prior screening exam, but older mammograms were available at most centers. How these were included in the hanging protocol varied. These differences might have added to the differential misclassification as they may have influenced how the radiologists classified the exams at the different centers.

\subsubsection{Confounding}

A confounder is a variable that affects both the exposure/independent variable and the outcome/dependent variable in such a way that a false association may appear (Figure 5.4) (230). In the OVVV-study, the exposure variable is screening technique, DBT+SM versus $\mathrm{DM}$, and the outcome variables are recall, cancer detection and histopathological findings of the cancers diagnosed. The only factor influencing the exposure variable is county of residence, whereas factors influencing the outcome variable mainly include risk factors for breast cancer (age and genes, hormonal/biological factors and mammographic density). A confounding factor in this study would therefore be a factor that both affects the county of residence as well as risk factors for breast cancer. Sociodemographic factors, such as educational level and immigration history may be such factors. 


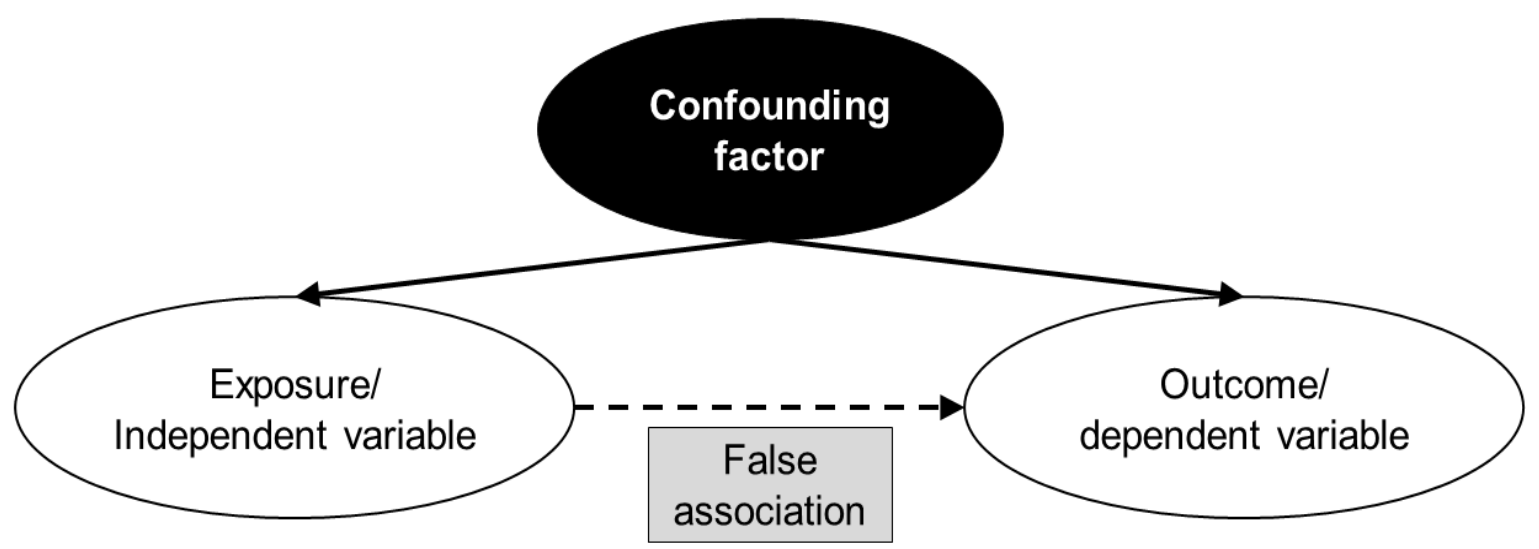

Figure 5.4. Confounding.

\subsubsection{External validity}

External validity refers to the generalizability of the study results; how conclusions drawn about the studied population are valid also for a more general target population (234). The OVVV-study was performed within a limited geographical area. Even if all women screened in this area were included in the study, the results may not necessarily be valid for other populations in Norway or internationally, particularly screening programs with different screening interval, or programs with single reading. Additionally, the study design as such with the study and control groups screened at different geographical locations and with equipment from different vendors, may limit the external generalizability. Further, the study group was screened with $\mathrm{DBT}+\mathrm{SM}$, which may limit generalizability to screening with $\mathrm{DBT}+\mathrm{DM}$; even though overall performance of SM in combination with DBT is demonstrated to be non-inferior to DBT+DM, individual differences may apply. However, the study sample was large and the results were comparable to results from other studies, hence, the external validity is considered satisfactory compared to programs with similar screening logistics.

The external validity is debatable for the review study. As described in the Introduction (section 1.6.3), several factors affect the results of the review and the proportion of cancers classified as missed. The more information available to the reviewers and the less comparable the review process is to a normal screening setting, the higher proportions of missed cancers are generally observed. Lack of standardization across programs and studies further hampers external generalizability and makes comparison of studies and review processes challenging $(140,197-199,201)$. When planning a radiological review study, the design must be related to the purpose of the study and the desired level of external generalizability. If the main purpose of the study is to evaluate the quality of the screening program and radiologists, a design simulating an ordinary screening setting with 
blinded reviews, preferentially with a mixture of negative mammograms among the interval cancer cases, might be preferred. If the purpose is mainly for education and selfassessment, an informed review is probably the most appropriate.

The study design of our review study was a fully informed, consensus based review of diagnostic and prior screening mammograms, which is considered to yield the highest proportion of missed cancer. The external generalizability of our results is thus limited, at least to studies with a different design. We chose this design because we wanted to include mammograms and radiologists from all breast centers in Norway and this design appeared most suitable for this purpose. Further, an educational perspective was important: we wanted to raise the awareness and improve knowledge among radiologists, and in that respect, a fully informed review was considered the most effective. As local radiologists not participating in the consensus panel were invited to the review as observers, the educational effects might have applied to them as well as the reviewers in the panel.

In addition, program characteristics as recall rate and screening interval might have impact on the classification into the different review categories. The threshold between what is considered as missed (should have been recalled) or minimal signs (recall possible, but not mandatory), probably differs with the accepted recall rates in different programs. Whether a program involves single or double reading, consensus/arbitration or practice early recall may also impact results from review. Further, as time between screening and diagnosis of interval cancer vary between classification categories (longest for true interval cancers) the proportions of missed and true cancers may vary in programs with different screening intervals. Finally, the review study only included DM, and not DBT images. Thus, the external validity of the results may be limited in screening programs with different recall rate, screening logistics and technique. 


\subsection{Ethical considerations}

In the invitation letter to mammographic screening, the women can read that relevant information about their screening examination is recorded by the Cancer Registry of Norway. Further, they are informed that this information is used for quality assurance, evaluation and research. The women are also informed that they have the right to refuse the storage of personal data related to a negative screening examination (3). In both studies in this project the Cancer Registry Regulation waived the requirement to obtain written informed consent for use of screening data for quality assurance and research (107).

Many studies have demonstrated increased cancer detection by screening with DBT compared with standard DM. Still, DBT is not a standard screening method in BreastScreen Norway or most other European breast cancer screening programs. This is mainly due to lack of evidence that DBT is a more effective screening tool, when taking into account the limited effect on interval cancer rates, probable increased detection of less aggressive cancers at screening and increased work load for radiologists. However, the question may be raised whether it is ethical not to introduce a screening method that probably increases the detection of a potentially lethal disease. For the target group, women attending screening, this may give rise to concern as they may feel they are not screened with the "best" method. On the other hand, implementing DBT in screening may potentially lead to overdiagnosis or overtreatment, as small, less aggressive tumors may be diagnosed, which also presents ethical challenges. A screening program for breast cancer finely balances benefits and harms, which is important, though sometimes challenging to communicate clearly to the target population and society in general.

As demonstrated in the review study and familiar to all radiologists, not only breast radiologists, pathological findings may be missed at screen reading, sometimes resulting in a less favorable prognosis or even death. An obvious ethical dilemma in that respect, is whether the patient has the right to be informed of a possible screening error. In the United Kingdom, a legislation has been passed, the "Duty of candour", which states that a woman has the right to be informed if an interval cancer results from an obvious screening error (missed cancer). However, a prerequisite is that there are obvious abnormal findings, and all reviewing radiologists have to agree on that recall should have been performed and classify the case as a "noticeable safety incident" (235). It has been stated that the proportion of interval cancers classified as false negatives using this classification amounts to 1-3\% (236), thus considerably less than the $22-24 \%$ missed cancers reported in our study. Similar differences have also been demonstrated in a study from BreastScreen Norway (201). This 
may illustrate the divergence between what is "technically missed", in a study situation in which the reviewers scrutinize the images to detect even minor learning points, and what is "clinically missed" with obvious negative health effects for the affected woman.

Communicating only the "technically missed" results may lead to reduced trust in the screening program among the target population and health authorities, and it may be difficult to convey the concept and limitations of screening to the public.

Another dilemma that has been proposed regarding radiological reviews, is whether performing reviews for academic and learning purposes only, is ethical. In an Irish report, an expert group stated that radiological reviews of interval cancer only should be allowed to conduct patient-requested case reviews of interval cancer, but not program-based reviews for educational purpose unless images were sufficiently blinded (236). However, in my opinion, and demonstrated by our study, informed reviews are important contributions to quality assurance and improvement within the screening program, and certain aspects might be lost if the review is fully blinded to clinical information and supplementary imaging. It might thus be argued the other way round, that hampering informed reviews might be unethical as important measures to improve the quality of mammographic screening cannot be taken.

\subsection{Clinical implications and future perspectives}

\subsubsection{Screening with DBT}

The overall aim of this $\mathrm{PhD}$ project was to investigate ways to improve early detection of breast cancers, and improve was defined as increased detection of clinically significant or "killing" cancers. Herein is an anticipated reduction in interval cancer. Our study confirmed that DBT is associated with increased cancer detection at screening, but not with increased detection of aggressive cancer, and not with a reduction of the more biologically aggressive interval cancers.

The gold standard for evaluation of screening effectiveness is the impact on mortality. As most breast cancer patients, including those who eventually die from the disease, survive for a long time, mortality analyses are dependent on long observation. Hence, conclusions on the effect of DBT on breast cancer mortality compared with DM cannot yet be drawn. However, earlier detection of small cancers may have a positive impact on morbidity, as less patients may receive less extensive or burdensome treatment $(237,238)$. 
Introducing DBT in screening in BreastScreen Norway would probably increase cancer detection, in particular in the prevalent round. Although no difference in recall rate between DBT and DM was demonstrated in our study, other studies from BreastScreen Norway have demonstrated reduced recall rates for screening with DBT $(64,161)$. The result of increased cancer detection and reduced recall rate is a reduction in rates of false positives, which is beneficial for women and society. However, the beneficial effects of DBT in screening must be balanced with possible harms such as increased workload related to increased interpretation time and the increased number of patients, as well as overdiagnosis. Further, screening with DBT increases requirements for data storage capacity markedly due to the large number of images in one exam. Additionally, training of the radiologists in screenreading with DBT as well as focus on hanging protocols and workflow is required to standardize screening and obtain an effective screening logistics.

Future focus on DBT as a screening tool may include studies on the long-term cancer detection and recall rates over several screening rounds, and in the end mortality rates. Further, more and larger studies aimed at analyzing interval cancer rates for screening with DBT are needed, preferably using aggregated data in order to obtain sufficiently powered analyses. Moreover, exploration of strategies to further increase personalization of breast cancer treatment may reduce the harm of overdiagnosis; if less or no treatment, combined with customized surveillance could be offered to patients with little or no risk of fatal disease, high detection of even small and less aggressive tumors might be beneficial. With that in mind, and by the rapidly evolving field of artificial intelligence (AI) in mammography and mammographic screening, I suggest that DBT in combination with synthetic mammograms might be suitable for breast cancer screening in the future. However, it is likely that a development of $\mathrm{Al}$ and introduction to screening will improve screening with standard DM as well.

\subsubsection{Interval cancer}

Both studies in this project confirmed that interval cancer has less favorable tumor characteristics compared with screen-detected cancer. Thus, a reduction of interval cancer rates is desired. Our study did not observe any beneficial decline in interval cancer from DBT, even if the rate of screen-detected cancer was 50\% higher for screening with DBT+SM compared with standard DM. However, the study was not powered to identify statistical significance at the observed rates. 
Higher mammographic density was observed in interval cancer compared with screendetected cancer, which is also observed in other studies $(190,239,240)$, occult interval cancers in particular. Program-related strategies to reduce the interval cancer rates in a screening program may include supplementary screening methods in groups more likely to be diagnosed with interval cancer, as women with dense breasts. The most promising technique in that respect is MRI, and studies have demonstrated reduced interval cancer rates for women with dense breasts screened with supplementary MRI compared with women screened with DM only $(73,75,76)$. However, the association between increased detection by supplementary screening techniques and reduction of - most preferably symptomatic interval cancer, has not been fully explored, and may be subject of future investigation. However, our results along with results from other studies, have not strengthened the evidence of DBT as such a supplementary tool.

Optimization of the screening process is essential to obtain high quality screening, and thereby potentially reduce the frequency of interval cancer. In addition to focusing on the screen-readers' performance, including education, reviews and satisfactory reading conditions, focusing on image quality might be crucial in that respect. Important factors for optimal image interpretation include technical parameters associated with image acquisition, post-processing and positioning of the breast. Sub-optimal image quality may be associated with increased recall rate, frequency of interval cancer and cancers missed at screening (33, $241,242)$.

Interval cancer is most frequently diagnosed due to clinical symptoms. However, some are diagnosed in an asymptomatic phase as an incidental finding in other radiological modalities, or as a result of an inter-screening mammography. These are cancers that otherwise most likely would have been diagnosed as screen-detected cancer in the next screening round. Based on the findings in the review study, they most probably have more favorable characteristics than symptomatic interval cancers. Interval cancer is sometimes considered as a negative effect of screening. However, it must be emphasized that the interval cancer is not caused by screening as such, the cancer would become symptomatic regardless of whether the woman attended the screening program. Further, in studies, interval cancer have comparable prognostic and predictive characteristics to cancers detected outside screening $(193,211,212)$. 


\subsubsection{What can radiologists learn from the review?}

The majority of visible abnormalities on prior screening mammograms presented as asymmetries. Thus a special focus on asymmetries seems reasonable, with the knowledge that asymmetries usually represent benign findings. However, by increasing their focus, radiologists may gain experience and confidence in the evaluation of which asymmetries potentially become clinically significant cancers. Additionally, even if calcifications represented the least frequent mammographic findings at prior screening for invasive screendetected and interval cancer, this category more frequently represented histological grade 3 cancer. Hence, increased attention to calcifications on mammograms may be reasonable, both regarding perception and interpretation, as well as image quality.

Interpretation of screening mammograms differs significantly from reading mammograms in a clinical setting. Clinical information in screening is sparse, the presence of abnormal findings is minor - the majority of screening mammograms are negative - and time spent reading each exam is short; screen-reading is usually performed in batches. The decision-making process on whether to recall or not is often based on experience, intuition and firstimpressions, rather than thorough analytic processes. Further, the decision whether to recall the women must be made within the frames of the accepted recall rates $(3-5 \%$ in BreastScreen Norway (226) and 5-7\% according to the European guidelines (23)) as well as the risk for a false positive recall. This as opposed to a clinical setting, in which women are referred due to a clinical problem that is expected to be solved.

With that in mind, our findings of visible abnormalities on prior screening mammograms in half of the reviewed cases, with a variable potential for earlier diagnosis, should not be interpreted as if the radiologists made mistakes when screening half of the later cancer cases within the program. As I see it, an important way to improve the sensitivity and specificity of the screen-readers, and thereby improve early detection of the clinical significant cancers, is to continuously evaluate radiologist performance. In that respect, reviews are an important tool. By characterizing, classifying and analyzing findings from the different categories of screen-detected and interval cancers, learning from mistakes and evaluation of current practice is possible. Further, the procedures involved in review studies may be integrated as part of every-day practice as self-audits, or as more formal audits on a regular basis. As described earlier, radiologists in the United Kingdom are obliged to perform audits of interval cancer by exploring whether the cancer was visible at prior screening and if so, whether the screening process may be classified as unsatisfactory/screening error (235). The advantage of an organized or obligatory audit is that radiologists are forced to evaluate 
their performance and face their possible errors. On the other hand, if the patients have the right to be informed whether the interval cancer was classified as a screening error, such formal audits may possibly restrict an honest evaluation, due to potential fear of legal consequences. In my opinion, from a radiologist's view, the best strategy for improving early detection of clinically significant cancers is an informed review strategy, and acceptance of a higher proportion of cancers classified as missed.

In my opinion, it is essential that screen-readers participate in work-up at recall assessment, which is also stated in the Quality Manual of BreastScreen Norway (108). Further, a greater learning effect may result from radiologists performing work-up on their own positive screens, though not always possible in everyday practice. Still, if occasionally feasible as a self-audit, I think this would be very valuable for continuous quality improvement in breast cancer screening.

\subsubsection{Artificial intelligence - future strategies to reduce the incidence of "killing cancers"}

Artificial intelligence ( $\mathrm{Al}$ ) based on deep learning is anticipated to play a major role in breast cancer screening and diagnostics in the upcoming years. Studies have suggested that Al performs as well as the average of several radiologists in screen reading (243), and that $\mathrm{Al}$ has the potential to reduce interval cancer rates (244). However, studies on Al in breast cancer screening are mainly reader studies on cancer-enriched material, and prospective studies on the performance of $\mathrm{Al}$ in everyday screening setting are needed. Topics of interest are whether Al has the potential to support or replace radiologists in screen-reading and whether an Al-system is able to detect cancers missed at screening by radiologists, thereby reducing the proportion of missed cancer at screening and also the interval cancer rates. Further, Al-integrated models of mammographic findings including mammographic density, and individual risk factors for breast cancer, may pave the way for more personalized breast cancer screening and treatment in the future. 


\section{Conclusions}

The main conclusions of the studies included in this thesis are:

1) An almost $50 \%$ increase in detection of cancer for women screened with $D B T+S M$ compared to women screened with DM was observed. Further, increased rates of DCIS, invasive tubular carcinomas, histologically grade 1 invasive cancer and invasive cancers with tumor diameter $<10 \mathrm{~mm}$ were observed for screening with DBT+SM compared with DM.

2) No differences in rates or characteristics of interval cancer were observed for women screened with DBT+SM versus DM. Further, the recall rate, cancer detection rate and rates and proportions of small and low grade invasive cancer declined at subsequent screening with DM compared to baseline screening with $\mathrm{DBT}+\mathrm{SM}$. No differences were observed in early outcome measures at baseline and consecutive screening for women screened with DM in both rounds.

In summary (I): We were not able to demonstrate that screening with DBT+SM increased detection of clinically significant cancers ("killing cancers"). Future meta-analyses and large prospective studies of short and long-term effects of screening with DBT are needed to make a decision on whether to recommend DBT in screening, based on scientific evidence.

3) In a fully informed radiological review of mammograms from screen-detected and interval cancer, $22-24 \%$ were classified as missed at prior screening, whereas in $46-48 \%$ no abnormalities were considered visible at the eventual cancer site on prior screening mammograms. Less favorable tumor characteristics and higher mammographic density were observed in interval cancer compared with screen-detected cancer.

4) Among cancers with visible findings at prior screening, both findings usually associated with malignancy (irregular spiculated masses) and findings more frequently associated with benign findings as one-plane asymmetries, were observed.

In summary (II): A reduction of missed cancer at screening may reduce the incidence of interval cancer. This may be achieved by program-related strategies as implementation of different screening intervals, supplementary screening methods or artificial intelligence to women at high risk for interval cancer, like women with mammographic dense breasts. However, radiologists are as yet the mainstay in screen-reading. Cancers are missed at screening due to both misperception and misinterpretation as demonstrated by our studies. Optimization of screening conditions and attention to the decision-making process in screenreading by radiologists may reduce the missed cancers at screening. In that respect, regular 
self-assessment and self-audits of screening cases may be useful, as well as use of Al to support the decision making.

In addition, the probable future increase in use of personalized treatment strategies and artificial intelligence may revolutionize both breast cancer screening and radiologists' work. In that respect, the results of this $\mathrm{PhD}$ project may contribute in preparing the ground for implementation of new methods as well as describing the present of breast cancer screening and diagnostics.

Planned future work as extension of the studies in this PhD project includes radiological review of the screen-detected and interval cancer in the OVVV-study. In addition, further studies comparing radiologist's performance in both studies with performance of artificial intelligence are planned in a post doc. 


\section{References}

1. IARC. Global Cancer Observatory Lyon, France.Available from: http://gco.iarc.frl. 2. Cancer Registry of Norway. Cancer in Norway 2019. Cancer incidence, mortality, survival and prevalence in Norway. Oslo,2020.

3. Hofvind S, Tsuruda K, Mangerud G, Ertzaas A, Holen A, Pedersen K, et al. The Norwegian Breast Cancer Screening Program, 1996-2016: Celebrating 20 years of organised mammographic screening. Cancer in Norway 2016 - Cancer incidence, mortality, survival and prevalence in Norway: Cancer Registry of Norway; 2017.

4. IARC. Breast Cancer Screening IARC Handbook of Cancer Prevention Volume 15. Lyon, France,2016.

5. Sebuodegard S, Botteri E, Hofvind S. Breast Cancer Mortality After Implementation of Organized Population-Based Breast Cancer Screening in Norway. J Natl Cancer Inst. 2020;112(8):839-46.

6. Saadatmand S, Bretveld R, Siesling S, Tilanus-Linthorst MM. Influence of tumour stage at breast cancer detection on survival in modern times: population based study in 173,797 patients. BMJ. 2015;351:h4901.

7. Boyd NF, Guo H, Martin LJ, Sun L, Stone J, Fishell E, et al. Mammographic density and the risk and detection of breast cancer. N Engl J Med. 2007;356(3):227-36.

8. Pettersson A, Graff RE, Ursin G, Santos Silva ID, McCormack V, Baglietto L, et al. Mammographic density phenotypes and risk of breast cancer: a meta-analysis. J Natl Cancer Inst. 2014;106(5).

9. McCormack VA, dos Santos Silva I. Breast density and parenchymal patterns as markers of breast cancer risk: a meta-analysis. Cancer Epidemiol Biomarkers Prev. 2006;15(6):1159-69.

10. Roman M, Sakshaug S, Graff-Iversen S, Vangen S, Weiderpass E, Ursin G, et al. Postmenopausal hormone therapy and the risk of breast cancer in Norway. Int J Cancer. 2016;138(3):584-93.

11. James JJ, Evans AJ. Grainger and Allison's Diagnostic Radiology2021.

12. Ellis IO, Lee AH, Pinder SE, Rakha EA. Tumors of the breast. Diagnostic Histopathology of Tumors: Elsevier; 2021. p. 1119-210.

13. Goncalves R, Warner WA, Luo J, Ellis MJ. New concepts in breast cancer genomics and genetics. Breast Cancer Res. 2014;16(5):460.

14. Badve SS, Gokmen-Polar Y. Ductal carcinoma in situ of breast: update 2019. Pathology. 2019;51(6):563-9.

15. Cowell CF, Weigelt B, Sakr RA, Ng CK, Hicks J, King TA, et al. Progression from ductal carcinoma in situ to invasive breast cancer: revisited. Mol Oncol. 2013;7(5):859-69.

16. Sanders ME, Schuyler PA, Simpson JF, Page DL, Dupont WD. Continued observation of the natural history of low-grade ductal carcinoma in situ reaffirms proclivity for local recurrence even after more than 30 years of follow-up. Mod Pathol. 2015;28(5):662-9. 17. Chootipongchaivat S, van Ravesteyn NT, Li X, Huang H, Weedon-Fekjaer H, Ryser $\mathrm{MD}$, et al. Modeling the natural history of ductal carcinoma in situ based on population data. Breast Cancer Res. 2020;22(1):53.

18. Weedon-Fekjaer H, Li X, Lee S. Estimating the natural progression of non-invasive ductal carcinoma in situ breast cancer lesions using screening data. J Med Screen. 2020:969141320945736.

19. Wilkinson L, Thomas V, Sharma N. Microcalcification on mammography: approaches to interpretation and biopsy. Br J Radiol. 2017;90(1069):20160594.

20. Burstein HJ, Polyak K, Wong JS, Lester SC, Kaelin CM. Ductal carcinoma in situ of the breast. N Engl J Med. 2004;350(14):1430-41. 
21. Johansson ALV, Trewin CB, Fredriksson I, Reinertsen KV, Russnes H, Ursin G. In modern times, how important are breast cancer stage, grade and receptor subtype for survival: a population-based cohort study. Breast Cancer Res. 2021;23(1):17.

22. Goldhirsch A, Winer EP, Coates AS, Gelber RD, Piccart-Gebhart M, Thurlimann B, et al. Personalizing the treatment of women with early breast cancer: highlights of the St Gallen International Expert Consensus on the Primary Therapy of Early Breast Cancer 2013. Ann Oncol. 2013;24(9):2206-23.

23. Perry N, Broeders M, de Wolf C, Tornberg S, Holland R, von Karsa L. European guidelines for quality assurance in breast cancer screening and diagnosis. Brussels, Belgium: European Communities; 2006.

24. Mazari FAK, Sharma N, Reid D, Horgan K. The need for triple assessment and predictors for diagnosis of breast cancer in patients $<40$ years of age. Clin Radiol. 2018;73(8):758 e19- e25.

25. Lehman CD, Lee AY, Lee Cl. Imaging management of palpable breast abnormalities. AJR Am J Roentgenol. 2014;203(5):1142-53.

26. Årsrapport Nasjonalt kvalitetsregister for brystkreft 2019. Available from: https://www.kvalitetsregistre.no/sites/default/files/202102/\%C3\%85rsrapport\%202019\%20Nasjonalt\%20kvalitetsregister\%20for\%20brystkreft.pdf. 27. Nasjonalt handlingsprogram for brystkreft: Norwegian Directorate for health; 2020 Available from: https://www.helsedirektoratet.no/retningslinjer/brystkreft-handlingsprogram. 28. Vyborny $\mathrm{CJ}$, Schmidt RA. Mammography as a radiographic examination: an overview. Radiographics. 1989;9(4):723-64.

29. Feig SA, Yaffe MJ. Digital mammography. Radiographics. 1998;18(4):893-901.

30. Vigeland E, Klaasen H, Klingen TA, Hofvind S, Skaane P. Full-field digital mammography compared to screen film mammography in the prevalent round of a population-based screening programme: the Vestfold County Study. Eur Radiol. 2008;18(1):183-91.

31. Skaane P. Studies comparing screen-film mammography and full-field digital mammography in breast cancer screening: updated review. Acta Radiol. 2009;50(1):3-14. 32. Hendrick RE, Ikeda D, Miyake KK. Breast imaging: The requisites: Elsevier; 2017. 33. Boita J, van Engen RE, Mackenzie A, Tingberg A, Bosmans H, Bolejko A, et al. How does image quality affect radiologists' perceived ability for image interpretation and lesion detection in digital mammography? Eur Radiol. 2021.

34. Sechopoulos I. A review of breast tomosynthesis. Part I. The image acquisition process. Med Phys. 2013;40(1):014301.

35. Sechopoulos I. A review of breast tomosynthesis. Part II. Image reconstruction, processing and analysis, and advanced applications. Med Phys. 2013;40(1):014302.

36. Michell MJ, Batohi B. Role of tomosynthesis in breast imaging going forward. Clin Radiol. 2018;73(4):358-71.

37. Chan HP, Helvie MA, Hadjiiski L, Jeffries DO, Klein KA, Neal CH, et al. Characterization of Breast Masses in Digital Breast Tomosynthesis and Digital Mammograms: An Observer Performance Study. Acad Radiol. 2017;24(11):1372-9.

38. Gennaro G, Bernardi D, Houssami N. Radiation dose with digital breast tomosynthesis compared to digital mammography: per-view analysis. Eur Radiol. 2018;28(2):573-81.

39. Zackrisson S, Lang K, Rosso A, Johnson K, Dustler M, Fornvik D, et al. One-view breast tomosynthesis versus two-view mammography in the Malmo Breast Tomosynthesis Screening Trial (MBTST): a prospective, population-based, diagnostic accuracy study. Lancet Oncol. 2018;19(11):1493-503.

40. Skaane P, Bandos Al, Eben EB, Jebsen IN, Krager M, Haakenaasen U, et al. Twoview digital breast tomosynthesis screening with synthetically reconstructed projection images: comparison with digital breast tomosynthesis with full-field digital mammographic images. Radiology. 2014;271(3):655-63. 
41. Zuckerman SP, Conant EF, Keller BM, Maidment AD, Barufaldi B, Weinstein SP, et al. Implementation of Synthesized Two-dimensional Mammography in a Population-based Digital Breast Tomosynthesis Screening Program. Radiology. 2016;281(3):730-6.

42. Aase HS, Holen AS, Pedersen K, Houssami N, Haldorsen IS, Sebuodegard S, et al. A randomized controlled trial of digital breast tomosynthesis versus digital mammography in population-based screening in Bergen: interim analysis of performance indicators from the To-Be trial. Eur Radiol. 2019;29(3):1175-86.

43. Abdullah P, Alabousi M, Ramadan S, Zawawi I, Zawawi M, Bhogadi Y, et al. Synthetic 2D Mammography Versus Standard 2D Digital Mammography: A Diagnostic Test Accuracy Systematic Review and Meta-Analysis. AJR Am J Roentgenol. 2020.

44. Zuckerman SP, Sprague BL, Weaver DL, Herschorn SD, Conant EF. Multicenter Evaluation of Breast Cancer Screening with Digital Breast Tomosynthesis in Combination with Synthetic versus Digital Mammography. Radiology. 2020;297(3):545-53.

45. Bernardi D, Macaskill P, Pellegrini M, Valentini M, Fanto C, Ostillio L, et al. Breast cancer screening with tomosynthesis (3D mammography) with acquired or synthetic 2D mammography compared with 2D mammography alone (STORM-2): a population-based prospective study. Lancet Oncol. 2016;17(8):1105-13.

46. Garayoa J, Chevalier M, Castillo M, Mahillo-Fernandez I, Amallal El Ouahabi N, Estrada C, et al. Diagnostic value of the stand-alone synthetic image in digital breast tomosynthesis examinations. Eur Radiol. 2018;28(2):565-72.

47. Zuley ML, Guo B, Catullo VJ, Chough DM, Kelly AE, Lu AH, et al. Comparison of twodimensional synthesized mammograms versus original digital mammograms alone and in combination with tomosynthesis images. Radiology. 2014;271(3):664-71.

48. Niklason LT, Christian BT, Niklason LE, Kopans DB, Castleberry DE, Opsahl-Ong $\mathrm{BH}$, et al. Digital tomosynthesis in breast imaging. Radiology. 1997;205(2):399-406.

49. Poplack S. Breast Tomosynthesis: Clinical Evidence. Radiol Clin North Am. 2017;55(3):475-92.

50. Gennaro G, Toledano A, di Maggio C, Baldan E, Bezzon E, La Grassa M, et al. Digital breast tomosynthesis versus digital mammography: a clinical performance study. Eur Radiol. 2010;20(7):1545-53.

51. Fornvik D, Zackrisson S, Ljungberg O, Svahn T, Timberg P, Tingberg A, et al. Breast tomosynthesis: Accuracy of tumor measurement compared with digital mammography and ultrasonography. Acta Radiol. 2010;51(3):240-7.

52. Svahn T, Andersson I, Chakraborty D, Svensson S, Ikeda D, Fornvik D, et al. The diagnostic accuracy of dual-view digital mammography, single-view breast tomosynthesis and a dual-view combination of breast tomosynthesis and digital mammography in a freeresponse observer performance study. Radiat Prot Dosimetry. 2010;139(1-3):113-7.

53. Gur D, Abrams GS, Chough DM, Ganott MA, Hakim CM, Perrin RL, et al. Digital breast tomosynthesis: observer performance study. AJR Am J Roentgenol. 2009;193(2):58691.

54. Gilbert FJ, Tucker L, Gillan MG, Willsher P, Cooke J, Duncan KA, et al. The TOMMY trial: a comparison of TOMosynthesis with digital MammographY in the UK NHS Breast Screening Programme--a multicentre retrospective reading study comparing the diagnostic performance of digital breast tomosynthesis and digital mammography with digital mammography alone. Health Technol Assess. 2015;19(4):i-xxv, 1-136.

55. Gilbert FJ, Tucker L, Gillan MG, Willsher P, Cooke J, Duncan KA, et al. Accuracy of Digital Breast Tomosynthesis for Depicting Breast Cancer Subgroups in a UK Retrospective Reading Study (TOMMY Trial). Radiology. 2015;277(3):697-706.

56. Greenberg JS, Javitt MC, Katzen J, Michael S, Holland AE. Clinical performance metrics of 3D digital breast tomosynthesis compared with 2D digital mammography for breast cancer screening in community practice. AJR Am J Roentgenol. 2014;203(3):687-93.

57. Conant EF, Beaber EF, Sprague BL, Herschorn SD, Weaver DL, Onega T, et al. Breast cancer screening using tomosynthesis in combination with digital mammography compared to digital mammography alone: a cohort study within the PROSPR consortium. Breast Cancer Res Treat. 2016;156(1):109-16. 
58. Friedewald SM, Rafferty EA, Rose SL, Durand MA, Plecha DM, Greenberg JS, et al. Breast cancer screening using tomosynthesis in combination with digital mammography. JAMA. 2014;311(24):2499-507.

59. Bernardi D, Ciatto S, Pellegrini M, Anesi V, Burlon S, Cauli E, et al. Application of breast tomosynthesis in screening: incremental effect on mammography acquisition and reading time. Br J Radiol. 2012;85(1020):e1174-8.

60. Skaane P, Bandos AI, Niklason LT, Sebuodegard S, Osteras BH, Gullien R, et al. Digital Mammography versus Digital Mammography Plus Tomosynthesis in Breast Cancer Screening: The Oslo Tomosynthesis Screening Trial. Radiology. 2019;291(1):23-30.

61. Romero Martin S, Raya Povedano JL, Cara Garcia M, Santos Romero AL, Pedrosa Garriguet M, Alvarez Benito M. Prospective study aiming to compare 2D mammography and tomosynthesis + synthesized mammography in terms of cancer detection and recall. From double reading of 2D mammography to single reading of tomosynthesis. Eur Radiol.

2018;28(6):2484-91.

62. Ciatto S, Houssami N, Bernardi D, Caumo F, Pellegrini M, Brunelli S, et al. Integration of 3D digital mammography with tomosynthesis for population breast-cancer screening (STORM): a prospective comparison study. Lancet Oncol. 2013;14(7):583-9.

63. Caumo F, Zorzi M, Brunelli S, Romanucci G, Rella R, Cugola L, et al. Digital Breast Tomosynthesis with Synthesized Two-Dimensional Images versus Full-Field Digital Mammography for Population Screening: Outcomes from the Verona Screening Program. Radiology. 2018;287(1):37-46.

64. Hofvind S, Holen AS, Aase HS, Houssami N, Sebuodegard S, Moger TA, et al. Twoview digital breast tomosynthesis versus digital mammography in a population-based breast cancer screening programme (To-Be): a randomised, controlled trial. Lancet Oncol. 2019;20(6):795-805.

65. Pattacini P, Nitrosi A, Giorgi Rossi P, lotti V, Ginocchi V, Ravaioli S, et al. Digital Mammography versus Digital Mammography Plus Tomosynthesis for Breast Cancer Screening: The Reggio Emilia Tomosynthesis Randomized Trial. Radiology. 2018;288(2):375-85.

66. Candelaria RP, Hwang L, Bouchard RR, Whitman GJ. Breast ultrasound: current concepts. Semin Ultrasound CT MR. 2013;34(3):213-25.

67. Yi A, Jang MJ, Yim D, Kwon BR, Shin SU, Chang JM. Addition of Screening Breast US to Digital Mammography and Digital Breast Tomosynthesis for Breast Cancer Screening in Women at Average Risk. Radiology. 2021:203134.

68. Berg WA, Blume JD, Cormack JB, Mendelson EB, Lehrer D, Bohm-Velez M, et al. Combined screening with ultrasound and mammography vs mammography alone in women at elevated risk of breast cancer. JAMA. 2008;299(18):2151-63.

69. Houssami N, Ciatto S. The evolving role of new imaging methods in breast screening. Prev Med. 2011;53(3):123-6.

70. Rella R, Belli P, Giuliani M, Bufi E, Carlino G, Rinaldi P, et al. Automated Breast Ultrasonography (ABUS) in the Screening and Diagnostic Setting: Indications and Practical Use. Acad Radiol. 2018;25(11):1457-70.

71. Skaane P, Gullien R, Eben EB, Sandhaug M, Schulz-Wendtland R, Stoeblen F. Interpretation of automated breast ultrasound (ABUS) with and without knowledge of mammography: a reader performance study. Acta Radiol. 2015;56(4):404-12.

72. Leithner D, Moy L, Morris EA, Marino MA, Helbich TH, Pinker K. Abbreviated MRI of the Breast: Does It Provide Value? J Magn Reson Imaging. 2019;49(7):e85-e100.

73. Kuhl CK, Strobel K, Bieling H, Leutner C, Schild HH, Schrading S. Supplemental Breast MR Imaging Screening of Women with Average Risk of Breast Cancer. Radiology. 2017;283(2):361-70.

74. Mann RM, Cho N, Moy L. Breast MRI: State of the Art. Radiology. 2019;292(3):520-

36.

75. Bakker MF, de Lange SV, Pijnappel RM, Mann RM, Peeters PHM, Monninkhof EM, et al. Supplemental MRI Screening for Women with Extremely Dense Breast Tissue. N Engl J Med. 2019;381(22):2091-102. 
76. Veenhuizen SGA, de Lange SV, Bakker MF, Pijnappel RM, Mann RM, Monninkhof EM, et al. Supplemental Breast MRI for Women with Extremely Dense Breasts: Results of the Second Screening Round of the DENSE Trial. Radiology. 2021:203633.

77. Kuhl CK, Schrading S, Strobel K, Schild HH, Hilgers RD, Bieling HB. Abbreviated breast magnetic resonance imaging (MRI): first postcontrast subtracted images and maximum-intensity projection-a novel approach to breast cancer screening with MRI. J Clin Oncol. 2014;32(22):2304-10.

78. Comstock CE, Gatsonis C, Newstead GM, Snyder BS, Gareen IF, Bergin JT, et al. Comparison of Abbreviated Breast MRI vs Digital Breast Tomosynthesis for Breast Cancer Detection Among Women With Dense Breasts Undergoing Screening. JAMA. 2020;323(8):746-56.

79. Weinstein SP, Korhonen K, Cirelli C, Schnall MD, McDonald ES, Pantel AR, et al. Abbreviated Breast Magnetic Resonance Imaging for Supplemental Screening of Women With Dense Breasts and Average Risk. J Clin Oncol. 2020;38(33):3874-82.

80. Zamora K, Allen E, Hermecz B. Contrast mammography in clinical practice: Current uses and potential diagnostic dilemmas. Clin Imaging. 2021;71:126-35.

81. Fallenberg EM, Schmitzberger FF, Amer H, Ingold-Heppner B, Balleyguier C, Diekmann F, et al. Contrast-enhanced spectral mammography vs. mammography and MRI clinical performance in a multi-reader evaluation. Eur Radiol. 2017;27(7):2752-64.

82. Boyd NF, Martin LJ, Bronskill M, Yaffe MJ, Duric N, Minkin S. Breast tissue composition and susceptibility to breast cancer. J Natl Cancer Inst. 2010;102(16):1224-37. 83. Wolfe JN. Breast patterns as an index of risk for developing breast cancer. AJR Am J Roentgenol. 1976;126(6):1130-7.

84. Gram IT, Funkhouser E, Tabar L. The Tabar classification of mammographic parenchymal patterns. Eur J Radiol. 1997;24(2):131-6.

85. Osteras BH, Martinsen AC, Brandal SH, Chaudhry KN, Eben E, Haakenaasen U, et al. Classification of fatty and dense breast parenchyma: comparison of automatic volumetric density measurement and radiologists' classification and their inter-observer variation. Acta Radiol. 2016;57(10):1178-85.

86. Sickles E, D'Orsi CJ, Bassett LW, et al. ACR BI-RADS ${ }^{\circledR}$ Mammography. In: ACR BIRADS $®$ Atlas, Breast Imaging Reporting and Data System. Reston, VA, : American College of Radiology; 2013.

87. Spak DA, Plaxco JS, Santiago L, Dryden MJ, Dogan BE. BI-RADS((R)) fifth edition: A summary of changes. Diagn Interv Imaging. 2017;98(3):179-90.

88. Winkel RR, von Euler-Chelpin M, Nielsen M, Diao P, Nielsen MB, Uldall WY, et al. Inter-observer agreement according to three methods of evaluating mammographic density and parenchymal pattern in a case control study: impact on relative risk of breast cancer. BMC Cancer. 2015;15:274.

89. Alomaim W, O'Leary D, Ryan J, Rainford L, Evanoff M, Foley S. Variability of Breast Density Classification Between US and UK Radiologists. J Med Imaging Radiat Sci. 2019;50(1):53-61.

90. Gram IT, Bremnes Y, Ursin G, Maskarinec G, Bjurstam N, Lund E. Percentage density, Wolfe's and Tabar's mammographic patterns: agreement and association with risk factors for breast cancer. Breast Cancer Res. 2005;7(5):R854-61.

91. Astley SM, Harkness EF, Sergeant JC, Warwick J, Stavrinos P, Warren R, et al. A comparison of five methods of measuring mammographic density: a case-control study. Breast Cancer Res. 2018;20(1):10.

92. Harkness EF, Astley SM, Evans DG. Risk-based breast cancer screening strategies in women. Best Pract Res Clin Obstet Gynaecol. 2020;65:3-17.

93. Sturesdotter L, Sandsveden M, Johnson K, Larsson AM, Zackrisson S, Sartor H. Mammographic tumour appearance is related to clinicopathological factors and surrogate molecular breast cancer subtype. Sci Rep. 2020;10(1):20814.

94. Chang RW, Chuang SL, Hsu CY, Yen AM, Wu WY, Chen SL, et al. Precision Science on Incidence and Progression of Early-Detected Small Breast Invasive Cancers by Mammographic Features. Cancers (Basel). 2020;12(7). 
95. Burnside ES, Sickles EA, Bassett LW, Rubin DL, Lee CH, Ikeda DM, et al. The ACR BI-RADS experience: learning from history. J Am Coll Radiol. 2009;6(12):851-60.

96. Wadhwa A, Sullivan JR, Gonyo MB. Missed Breast Cancer: What Can We Learn? Curr Probl Diagn Radiol. 2016;45(6):402-19.

97. Marmot MG, Altman DG, Cameron DA, Dewar JA, Thompson SG, Wilcox M. The benefits and harms of breast cancer screening: an independent review. $\mathrm{Br} \mathrm{J}$ Cancer. 2013;108(11):2205-40.

98. Croswell JM, Ransohoff DF, Kramer BS. Principles of cancer screening: lessons from history and study design issues. Semin Oncol. 2010;37(3):202-15.

99. Wilson JMG, Jungner G. Principles and practice of screening for disease. Geneva: World Health Organization; 1968.

100. Helsedirektoratet. Styringsstrukturer og strategi for nasjonale screeningprogrammer. IS-2242. Oslo: Helsedirektoratet; 2014.

101. Gotzsche PC, Jorgensen KJ. Screening for breast cancer with mammography. Cochrane Database Syst Rev. 2013(6):CD001877.

102. Bulliard JL, Ducros C, Jemelin C, Arzel B, Fioretta G, Levi F. Effectiveness of organised versus opportunistic mammography screening. Ann Oncol. 2009;20(7):1199-202. 103. Hofvind S, Hovda T, Holen AS, Lee Cl, Albertsen J, Bjorndal H, et al. Digital Breast Tomosynthesis and Synthetic 2D Mammography versus Digital Mammography: Evaluation in a Population-based Screening Program. Radiology. 2018;287(3):787-94.

104. Hofvind S, Moshina N, Holen AS, Danielsen AS, Lee Cl, Houssami N, et al. Interval and Subsequent Round Breast Cancer in a Randomized Controlled Trial Comparing Digital Breast Tomosynthesis and Digital Mammography Screening. Radiology. 2021:203936.

105. Systembeskrivelse Nasjonalt system for innføring av nye metoder i spesialisthelsetjenesten - for bedre og tryggere pasientbehandling. 2013.

106. Three-dimensional digital breast tomosynthesis (DBT) in breast cancer screening: Norwegian Institute of Public Health; 2017 Available from:

https://nyemetoder.no/metoder/tredimensjonal-digital-brysttomosyntese-dbt-i-screening-forbrystkreft.

107. Ministry of Health and Care Services, Forskrift om innsamling og behandling av helseopplysninger i Kreftregisteret (The Cancer Registry Regulation), (2001).

108. Bjørndal H, Vigeland E, Aagedal GE, Østlie A, Aase HS, Brandal SHB, et al. Kvalitetsmanual i Mammografiprogrammet - retningslinjer for radiologi. Available from www.kreftregisteret.no/Generelt/Rapporter/Mammografiprogrammet/Kvalitet/. Cancer Registry of Norway; 2019.

109. Available from: www.kreftregisteret.no/screening/mammografiprogrammet/.

110. Paap E, Verbeek A, Puliti D, Broeders M, Paci E. Minor influence of self-selection bias on the effectiveness of breast cancer screening in case-control studies in the Netherlands. J Med Screen. 2011;18(3):142-6.

111. Broeders M, Moss S, Nystrom L, Njor S, Jonsson H, Paap E, et al. The impact of mammographic screening on breast cancer mortality in Europe: a review of observational studies. J Med Screen. 2012;19 Suppl 1:14-25.

112. Hofvind S, Ursin G, Tretli S, Sebuodegard S, Moller B. Breast cancer mortality in participants of the Norwegian Breast Cancer Screening Program. Cancer.

2013;119(17):3106-12.

113. Weedon-Fekjaer H, Romundstad PR, Vatten LJ. Modern mammography screening and breast cancer mortality: population study. BMJ. 2014;348:g3701.

114. Lee JM, Lowry KP, Cott Chubiz JE, Swan JS, Motazedi T, Halpern EF, et al. Breast cancer risk, worry, and anxiety: Effect on patient perceptions of false-positive screening results. Breast. 2020;50:104-12.

115. Schou Bredal I, Karesen R, Skaane P, Engelstad KS, Ekeberg O. Recall mammography and psychological distress. Eur J Cancer. 2013;49(4):805-11.

116. Roman M, Hubbard RA, Sebuodegard S, Miglioretti DL, Castells X, Hofvind S. The cumulative risk of false-positive results in the Norwegian Breast Cancer Screening Program: updated results. Cancer. 2013;119(22):3952-8. 
117. Hofvind S, Ponti A, Patnick J, Ascunce N, Njor S, Broeders M, et al. False-positive results in mammographic screening for breast cancer in Europe: a literature review and survey of service screening programmes. J Med Screen. 2012;19 Suppl 1:57-66.

118. Domingo L, Hofvind S, Hubbard RA, Roman M, Benkeser D, Sala M, et al. Crossnational comparison of screening mammography accuracy measures in U.S., Norway, and Spain. Eur Radiol. 2016;26(8):2520-8.

119. Roman M, Castells X, Hofvind S, von Euler-Chelpin M. Risk of breast cancer after false-positive results in mammographic screening. Cancer Med. 2016;5(6):1298-306.

120. von Euler-Chelpin M, Kuchiki M, Vejborg I. Increased risk of breast cancer in women with false-positive test: the role of misclassification. Cancer Epidemiol. 2014;38(5):619-22. 121. Hofvind S, Sagstad S, Sebuodegard S, Chen Y, Roman M, Lee Cl. Interval Breast Cancer Rates and Histopathologic Tumor Characteristics after False-Positive Findings at Mammography in a Population-based Screening Program. Radiology. 2018;287(1):58-67. 122. von Euler-Chelpin M, Risor LM, Thorsted BL, Vejborg I. Risk of breast cancer after false-positive test results in screening mammography. J Natl Cancer Inst. 2012;104(9):682-9. 123. Honig EL, Mullen LA, Amir T, Alvin MD, Jones MK, Ambinder EB, et al. Factors Impacting False Positive Recall in Screening Mammography. Acad Radiol. 2019;26(11):1505-12.

124. Hubbard RA, Kerlikowske K, Flowers Cl, Yankaskas BC, Zhu W, Miglioretti DL. Cumulative probability of false-positive recall or biopsy recommendation after 10 years of screening mammography: a cohort study. Ann Intern Med. 2011;155(8):481-92.

125. Falk RS, Hofvind S. Overdiagnosis in Mammographic Screening because of Competing Risk of Death. Cancer Epidemiol Biomarkers Prev. 2016;25(5):759-65.

126. Puliti D, Duffy SW, Miccinesi G, de Koning H, Lynge E, Zappa M, et al. Overdiagnosis in mammographic screening for breast cancer in Europe: a literature review. J Med Screen. 2012;19 Suppl 1:42-56.

127. Nelson HD, Pappas M, Cantor A, Griffin J, Daeges M, Humphrey L. Harms of Breast Cancer Screening: Systematic Review to Update the 2009 U.S. Preventive Services Task Force Recommendation. Ann Intern Med. 2016;164(4):256-67.

128. Falk RS, Hofvind S, Skaane P, Haldorsen T. Overdiagnosis among women attending a population-based mammography screening program. Int J Cancer. 2013;133(3):705-12. 129. Canelo-Aybar C, Ferreira DS, Ballesteros M, Posso M, Montero N, Sola I, et al. Benefits and harms of breast cancer mammography screening for women at average risk of breast cancer: A systematic review for the European Commission Initiative on Breast Cancer. J Med Screen. 2021:969141321993866.

130. Neal CH, Helvie MA. Overdiagnosis and Risks of Breast Cancer Screening. Radiol Clin North Am. 2021;59(1):19-27.

131. de Gelder R, Heijnsdijk EA, van Ravesteyn NT, Fracheboud J, Draisma G, de Koning $\mathrm{HJ}$. Interpreting overdiagnosis estimates in population-based mammography screening. Epidemiol Rev. 2011;33:111-21.

132. Autier $\mathrm{P}$, Boniol M. Mammography screening: A major issue in medicine. Eur $\mathrm{J}$ Cancer. 2018;90:34-62.

133. ECIBC. Recommendations from the European Breast Cancer Guidelines [updated 28/05/2020. Available from: https://healthcare-quality.jrc.ec.europa.eu/european-breastcancer-guidelines Accessed June 2020.

134. WHO: Breast cancer prevention and control Available from: https://www.who.int/cancer/detection/breastcancer/en/.

135. Arleo EK, Hendrick RE, Helvie MA, Sickles EA. Comparison of recommendations for screening mammography using CISNET models. Cancer. 2017;123(19):3673-80.

136. Kolb TM, Lichy J, Newhouse JH. Comparison of the performance of screening mammography, physical examination, and breast US and evaluation of factors that influence them: an analysis of 27,825 patient evaluations. Radiology. 2002;225(1):165-75.

137. Rafferty EA, Durand MA, Conant EF, Copit DS, Friedewald SM, Plecha DM, et al. Breast Cancer Screening Using Tomosynthesis and Digital Mammography in Dense and Nondense Breasts. JAMA. 2016;315(16):1784-6. 
138. Lowry KP, Coley RY, Miglioretti DL, Kerlikowske K, Henderson LM, Onega T, et al. Screening Performance of Digital Breast Tomosynthesis vs Digital Mammography in Community Practice by Patient Age, Screening Round, and Breast Density. JAMA Netw Open. 2020;3(7):e2011792.

139. Osteras BH, Martinsen ACT, Gullien R, Skaane P. Digital Mammography versus Breast Tomosynthesis: Impact of Breast Density on Diagnostic Performance in Populationbased Screening. Radiology. 2019;293(1):60-8.

140. Houssami N, Irwig L, Ciatto S. Radiological surveillance of interval breast cancers in screening programmes. Lancet Oncol. 2006;7(3):259-65.

141. Hoff SR, Myklebust TA, Lee $\mathrm{Cl}$, Hofvind S. Influence of Mammography Volume on Radiologists' Performance: Results from BreastScreen Norway. Radiology. 2019;292(2):28996.

142. Ekpo EU, Alakhras M, Brennan P. Errors in Mammography Cannot be Solved Through Technology Alone. Asian Pac J Cancer Prev. 2018;19(2):291-301.

143. Elmore JG, Jackson SL, Abraham L, Miglioretti DL, Carney PA, Geller BM, et al. Variability in interpretive performance at screening mammography and radiologists' characteristics associated with accuracy. Radiology. 2009;253(3):641-51.

144. The Mammography Quality Standards Act Final Regulations: FDA; [updated 20/08/2018. Available from: https://www.fda.gov/regulatory-information/search-fda-guidancedocuments/compliance-guidance-mammography-quality-standards-act-final-regulationspreparing-mqsa-inspections Accessed April 2021.

145. Backmann HA, Larsen M, Danielsen AS, Hofvind S. Time of day and mammographic reader performance in a population-based breast cancer screening programme. J Med Screen. 2020:969141320953206.

146. Hofvind S, Bennett RL, Brisson J, Lee W, Pelletier E, Flugelman A, et al. Audit feedback on reading performance of screening mammograms: An international comparison. J Med Screen. 2016;23(3):150-9.

147. Geertse TD, Paap E, van der Waal D, Duijm LEM, Pijnappel RM, Broeders MJM. Utility of Supplemental Training to Improve Radiologist Performance in Breast Cancer Screening: A Literature Review. J Am Coll Radiol. 2019;16(11):1528-46.

148. Miglioretti DL, Gard CC, Carney PA, Onega TL, Buist DS, Sickles EA, et al. When radiologists perform best: the learning curve in screening mammogram interpretation. Radiology. 2009;253(3):632-40.

149. Trieu PDY, Tapia K, Frazer H, Lee W, Brennan P. Improvement of Cancer Detection on Mammograms via BREAST Test Sets. Acad Radiol. 2019;26(12):e341-e7.

150. Lamb LR, Mohallem Fonseca M, Verma R, Seely JM. Missed Breast Cancer: Effects of Subconscious Bias and Lesion Characteristics. Radiographics. 2020;40(4):941-60.

151. Geller BM, Bogart A, Carney PA, Sickles EA, Smith R, Monsees B, et al. Educational interventions to improve screening mammography interpretation: a randomized controlled trial. AJR Am J Roentgenol. 2014;202(6):W586-96.

152. Tomosynthesis Mammographic Imaging Screening Trial (TMIST) Available from: https://clinicaltrials.gov/ct2/show/NCT03233191?term=dbt\&recrs=ab\&draw=3\&rank=17.

153. Prospective Trial of Digital Breast Tomosynthesis (DBT) in Breast Cancer Screening. (PROSPECTS) Available from:

https://clinicaltrials.gov/ct2/show/NCT03733106?term=dbt\&recrs=ab\&draw=2\&rank=3.

154. Marinovich ML, Hunter KE, Macaskill P, Houssami N. Breast Cancer Screening Using Tomosynthesis or Mammography: A Meta-analysis of Cancer Detection and Recall. J Natl Cancer Inst. 2018;110(9):942-9.

155. Gao Y, Moy L, Heller SL. Digital Breast Tomosynthesis: Update on Technology, Evidence, and Clinical Practice. Radiographics. 2021;41(2):321-37.

156. Skaane P, Bandos AI, Gullien R, Eben EB, Ekseth U, Haakenaasen U, et al. Comparison of digital mammography alone and digital mammography plus tomosynthesis in a population-based screening program. Radiology. 2013;267(1):47-56.

157. Bernardi D, Gentilini MA, De Nisi M, Pellegrini M, Fanto C, Valentini M, et al. Effect of implementing digital breast tomosynthesis (DBT) instead of mammography on population 
screening outcomes including interval cancer rates: Results of the Trento DBT pilot evaluation. Breast. 2020;50:135-40.

158. Skaane P, Bandos Al, Gullien R, Eben EB, Ekseth U, Haakenaasen U, et al. Prospective trial comparing full-field digital mammography (FFDM) versus combined FFDM and tomosynthesis in a population-based screening programme using independent double reading with arbitration. Eur Radiol. 2013;23(8):2061-71.

159. Houssami N, Bernardi D, Caumo F, Brunelli S, Fanto C, Valentini M, et al. Interval breast cancers in the 'screening with tomosynthesis or standard mammography' (STORM) population-based trial. Breast. 2018;38:150-3.

160. Skaane P, Sebuodegard S, Bandos Al, Gur D, Osteras BH, Gullien R, et al. Performance of breast cancer screening using digital breast tomosynthesis: results from the prospective population-based Oslo Tomosynthesis Screening Trial. Breast Cancer Res Treat. 2018;169(3):489-96.

161. Hovda T, Brandal SHB, Sebuodegard S, Holen AS, Bjorndal H, Skaane P, et al. Screening outcome for consecutive examinations with digital breast tomosynthesis versus standard digital mammography in a population-based screening program. Eur Radiol. 2019;29(12):6991-9.

162. Bahl M, Gaffney S, McCarthy AM, Lowry KP, Dang PA, Lehman CD. Breast Cancer Characteristics Associated with 2D Digital Mammography versus Digital Breast

Tomosynthesis for Screening-detected and Interval Cancers. Radiology. 2018;287(1):49-57. 163. Winter AM, Kazmi S, Hardy AK, Bennett DL. Comparison of interval breast cancers with 2D digital mammography versus 3D digital breast tomosynthesis in a large communitybased practice. Breast J. 2020;26(10):1953-9.

164. McDonald ES, Oustimov A, Weinstein SP, Synnestvedt MB, Schnall M, Conant EF. Effectiveness of Digital Breast Tomosynthesis Compared With Digital Mammography: Outcomes Analysis From 3 Years of Breast Cancer Screening. JAMA Oncol. 2016;2(6):73743.

165. Johnson K, Lang K, Ikeda DM, Akesson A, Andersson I, Zackrisson S. Interval Breast Cancer Rates and Tumor Characteristics in the Prospective Population-based Malmo Breast Tomosynthesis Screening Trial. Radiology. 2021:204106.

166. Houssami N, Zackrisson S, Blazek K, Hunter K, Bernardi D, Lang K, et al. Metaanalysis of prospective studies evaluating breast cancer detection and interval cancer rates for digital breast tomosynthesis versus mammography population screening. Eur $\mathrm{J}$ Cancer. 2021;148:14-23.

167. Houssami N, Hofvind S, Soerensen AL, Robledo KP, Hunter K, Bernardi D, et al. Interval breast cancer rates for digital breast tomosynthesis versus digital mammography population screening: An individual participant data meta-analysis. EClinical Medicine. 2021;34(April 2021).

168. Bulliard JL, Sasieni P, Klabunde C, De Landtsheer JP, Yankaskas BC, Fracheboud J. Methodological issues in international comparison of interval breast cancers. Int $\mathrm{J}$ Cancer. 2006;119(5):1158-63.

169. Andersen SB, Tornberg S, Lynge E, Von Euler-Chelpin M, Njor SH. A simple way to measure the burden of interval cancers in breast cancer screening. BMC Cancer. 2014;14:782.

170. Tornberg S, Kemetli L, Ascunce N, Hofvind S, Anttila A, Seradour B, et al. A pooled analysis of interval cancer rates in six European countries. Eur J Cancer Prev. 2010;19(2):87-93.

171. Houssami N, Hunter K. The epidemiology, radiology and biological characteristics of interval breast cancers in population mammography screening. NPJ Breast Cancer. 2017;3:12.

172. Larsen IK, Smastuen M, Johannesen TB, Langmark F, Parkin DM, Bray F, et al. Data quality at the Cancer Registry of Norway: an overview of comparability, completeness, validity and timeliness. Eur J Cancer. 2009;45(7):1218-31. 
173. Weber RJ, van Bommel RM, Louwman MW, Nederend J, Voogd AC, Jansen FH, et al. Characteristics and prognosis of interval cancers after biennial screen-film or full-field digital screening mammography. Breast Cancer Res Treat. 2016;158(3):471-83.

174. Carbonaro LA, Azzarone A, Paskeh BB, Brambilla G, Brunelli S, Calori A, et al. Interval breast cancers: absolute and proportional incidence and blinded review in a community mammographic screening program. Eur J Radiol. 2014;83(2):e84-91.

175. National Health Service Breast Screening Programme. National collation of breast interval cancer data. NHSBSP Occational Report 12/03. 2012.

176. Hofvind S, Yankaskas BC, Bulliard JL, Klabunde CN, Fracheboud J. Comparing interval breast cancer rates in Norway and North Carolina: results and challenges. J Med Screen. 2009;16(3):131-9.

177. Hofvind S, Vacek PM, Skelly J, Weaver DL, Geller BM. Comparing screening mammography for early breast cancer detection in Vermont and Norway. J Natl Cancer Inst. 2008;100(15):1082-91.

178. Bennett RL, Sellars SJ, Moss SM. Interval cancers in the NHS breast cancer screening programme in England, Wales and Northern Ireland. $\mathrm{Br} \mathrm{J}$ Cancer. 2011;104(4):571-7.

179. Warren R, Duffy S. Interval cancers as an indicator of performance in breast screening. Breast Cancer. 2000;7(1):9-18.

180. Burnside ES, Vulkan D, Blanks RG, Duffy SW. Association between Screening Mammography Recall Rate and Interval Cancers in the UK Breast Cancer Service Screening Program: A Cohort Study. Radiology. 2018;288(1):47-54.

181. Otten JD, Karssemeijer N, Hendriks JH, Groenewoud JH, Fracheboud J, Verbeek AL, et al. Effect of recall rate on earlier screen detection of breast cancers based on the Dutch performance indicators. J Natl Cancer Inst. 2005;97(10):748-54.

182. Blanks RG, Given-Wilson RM, Cohen SL, Patnick J, Alison RJ, Wallis MG. An analysis of 11.3 million screening tests examining the association between recall and cancer detection rates in the English NHS breast cancer screening programme. Eur Radiol. 2019;29(7):3812-9.

183. Messinger J, Crawford S, Roland L, Mizuguchi S. Review of Subtypes of Interval Breast Cancers With Discussion of Radiographic Findings. Curr Probl Diagn Radiol. 2019;48(6):592-8.

184. Kirsh VA, Chiarelli AM, Edwards SA, O'Malley FP, Shumak RS, Yaffe MJ, et al. Tumor characteristics associated with mammographic detection of breast cancer in the Ontario breast screening program. J Natl Cancer Inst. 2011;103(12):942-50.

185. Holm J, Humphreys K, Li J, Ploner A, Cheddad A, Eriksson M, et al. Risk factors and tumor characteristics of interval cancers by mammographic density. J Clin Oncol. 2015;33(9):1030-7.

186. Grassmann F, He W, Eriksson M, Gabrielson M, Hall P, Czene K. Interval breast cancer is associated with other types of tumors. Nat Commun. 2019;10(1):4648.

187. Hofvind S, Geller BM, Rosenberg RD, Skaane P. Screening-detected breast cancers: discordant independent double reading in a population-based screening program. Radiology. 2009;253(3):652-60.

188. Bellio G, Marion R, Giudici F, Kus S, Tonutti M, Zanconati F, et al. Interval Breast Cancer Versus Screen-Detected Cancer: Comparison of Clinicopathologic Characteristics in a Single-Center Analysis. Clin Breast Cancer. 2017;17(7):564-71.

189. Meshkat B, Prichard RS, Al-Hilli Z, Bass GA, Quinn C, O'Doherty A, et al. A comparison of clinical-pathological characteristics between symptomatic and interval breast cancer. Breast. 2015;24(3):278-82.

190. Domingo L, Sala M, Servitja S, Corominas JM, Ferrer F, Martinez J, et al. Phenotypic characterization and risk factors for interval breast cancers in a population-based breast cancer screening program in Barcelona, Spain. Cancer Causes Control. 2010;21(8):1155-64. 191. Bare M, Tora N, Salas D, Sentis M, Ferrer J, Ibanez J, et al. Mammographic and clinical characteristics of different phenotypes of screen-detected and interval breast cancers in a nationwide screening program. Breast Cancer Res Treat. 2015;154(2):403-15. 
192. Cheasley D, Li N, Rowley SM, Elder K, Mann GB, Loi S, et al. Molecular comparison of interval and screen-detected breast cancers. J Pathol. 2019;248(2):243-52.

193. Palka I, Kelemen G, Ormandi K, Lazar G, Nyari T, Thurzo L, et al. Tumor characteristics in screen-detected and symptomatic breast cancers. Pathol Oncol Res. 2008;14(2):161-7.

194. Rayson D, Payne JI, Abdolell M, Barnes PJ, Maclntosh RF, Foley T, et al. Comparison of clinical-pathologic characteristics and outcomes of true interval and screendetected invasive breast cancer among participants of a Canadian breast screening program: a nested case-control study. Clin Breast Cancer. 2011;11(1):27-32.

195. Wishart GC, Greenberg DC, Britton PD, Chou P, Brown CH, Purushotham AD, et al. Screen-detected vs symptomatic breast cancer: is improved survival due to stage migration alone? Br J Cancer. 2008;98(11):1741-4.

196. Mook S, Van 't Veer LJ, Rutgers EJ, Ravdin PM, van de Velde AO, van Leeuwen FE, et al. Independent prognostic value of screen detection in invasive breast cancer. J Natl Cancer Inst. 2011;103(7):585-97.

197. Gordon PB, Borugian MJ, Warren Burhenne LJ. A true screening environment for review of interval breast cancers: pilot study to reduce bias. Radiology. 2007;245(2):411-5. 198. Ciatto S, Catarzi S, Lamberini MP, Risso G, Saguatti G, Abbattista T, et al. Interval breast cancers in screening: the effect of mammography review method on classification. Breast. 2007;16(6):646-52.

199. Moberg K, Grundstrom H, Tornberg S, Lundquist H, Svane G, Havervall L, et al. Two models for radiological reviewing of interval cancers. J Med Screen. 1999;6(1):35-9.

200. de Rijke JM, Schouten LJ, Schreutelkamp JL, Jochem I, Verbeek AL. A blind review and an informed review of interval breast cancer cases in the Limburg screening programme, the Netherlands. J Med Screen. 2000;7(1):19-23.

201. Hofvind S, Skaane P, Vitak B, Wang H, Thoresen S, Eriksen L, et al. Influence of review design on percentages of missed interval breast cancers: retrospective study of interval cancers in a population-based screening program. Radiology. 2005;237(2):437-43.

202. Hoff SR, Abrahamsen AL, Samset JH, Vigeland E, Klepp O, Hofvind S. Breast cancer: missed interval and screening-detected cancer at full-field digital mammography and screen-film mammography-- results from a retrospective review. Radiology. 2012;264(2):378-86.

203. Hovda T, Holen AS, Lang K, Albertsen JL, Bjorndal H, Brandal SHB, et al. Interval and Consecutive Round Breast Cancer after Digital Breast Tomosynthesis and Synthetic 2D Mammography versus Standard 2D Digital Mammography in BreastScreen Norway. Radiology. 2020;294(2):256-64.

204. Hovda T, Tsuruda K, Hoff SR, Sahlberg KK, Hofvind S. Radiological review of prior screening mammograms of screen-detected breast cancer. Eur Radiol. 2021;31(4):2568-79.

205. Hovda T, Hoff SR, Larsen M, Romundstad L, Sahlberg KK, Hofvind S. True and Missed Interval Cancer in Organized Mammographic Screening: A Retrospective Review Study of Diagnostic and Prior Screening Mammograms. Acad Radiol. 2021.

206. Hofvind S, Geller B, Vacek PM, Thoresen S, Skaane P. Using the European guidelines to evaluate the Norwegian Breast Cancer Screening Program. Eur J Epidemiol. 2007;22(7):447-55.

207. Caumo F, Montemezzi S, Romanucci G, Brunelli S, Bricolo P, Cugola L, et al. Repeat Screening Outcomes with Digital Breast Tomosynthesis Plus Synthetic Mammography for Breast Cancer Detection: Results from the Prospective Verona Pilot Study. Radiology. 2021;298(1):49-57.

208. Nasjonalt handlingsprogram med retningslinjer for diagnostikk, behandling og oppfølging av pasienter med brystkreft: Helsedirektoratet; 2018. Available from:

https://nbcgblog.files.wordpress.com/2018/08/is-2736 brystkrefthandlingsprogram-11utgave.pdf.

209. Conant EF, Barlow WE, Herschorn SD, Weaver DL, Beaber EF, Tosteson ANA, et al. Association of Digital Breast Tomosynthesis vs Digital Mammography With Cancer Detection and Recall Rates by Age and Breast Density. JAMA Oncol. 2019;5(5):635-42. 
210. Domingo L, Salas D, Zubizarreta R, Bare M, Sarriugarte G, Barata T, et al. Tumor phenotype and breast density in distinct categories of interval cancer: results of populationbased mammography screening in Spain. Breast Cancer Res. 2014;16(1):R3.

211. Hofvind S, Holen A, Roman M, Sebuodegard S, Puig-Vives M, Akslen L. Mode of detection: an independent prognostic factor for women with breast cancer. J Med Screen. 2016;23(2):89-97.

212. Niraula S, Biswanger N, Hu P, Lambert P, Decker K. Incidence, Characteristics, and Outcomes of Interval Breast Cancers Compared With Screening-Detected Breast Cancers. JAMA Network Open. 2020;3(9):e2018179-e.

213. Li J, Ivansson E, Klevebring D, Tobin NP, Lindstrom LS, Holm J, et al. Molecular Differences between Screen-Detected and Interval Breast Cancers Are Largely Explained by PAM50 Subtypes. Clin Cancer Res. 2017;23(10):2584-92.

214. Li J, Holm J, Bergh J, Eriksson M, Darabi H, Lindstrom LS, et al. Breast cancer genetic risk profile is differentially associated with interval and screen-detected breast cancers. Ann Oncol. 2015;26(3):517-22.

215. Tsuruda KM, Hovda T, Bhargava S, Veierod MB, Hofvind S. Survival among women diagnosed with screen-detected or interval breast cancer classified as true, minimal signs, or missed through an informed radiological review. Eur Radiol. 2020.

216. Fong Y, Evans J, Brook D, Kenkre J, Jarvis P, Gower Thomas K. The incidence and 10-year survival of interval breast cancers in Wales. Clin Radiol. 2014;69(4):e168-72.

217. Hofvind S, Geller B, Skaane P. Mammographic features and histopathological findings of interval breast cancers. Acta Radiol. 2008;49(9):975-81.

218. Hoff SR, Samset JH, Abrahamsen AL, Vigeland E, Klepp O, Hofvind S. Missed and true interval and screen-detected breast cancers in a population based screening program. Acad Radiol. 2011;18(4):454-60.

219. Evans AJ, Kutt E, Record C, Waller M, Bobrow L, Moss S. Radiological and pathological findings of interval cancers in a multi-centre, randomized, controlled trial of mammographic screening in women from age 40-41 years. Clin Radiol. 2007;62(4):348-52. 220. Porter GJ, Evans AJ, Burrell HC, Lee AH, Ellis IO, Chakrabarti J. Interval breast cancers: prognostic features and survival by subtype and time since screening. J Med Screen. 2006;13(3):115-22.

221. Vitak B, Olsen KE, Manson JC, Arnesson LG, Stal O. Tumour characteristics and survival in patients with invasive interval breast cancer classified according to mammographic findings at the latest screening: a comparison of true interval and missed interval cancers. Eur Radiol. 1999;9(3):460-9.

222. Blanch J, Sala M, Ibanez J, Domingo L, Fernandez B, Otegi A, et al. Impact of risk factors on different interval cancer subtypes in a population-based breast cancer screening programme. PLoS One. 2014;9(10):e110207.

223. Mandelblatt JS, Stout NK, Schechter CB, van den Broek JJ, Miglioretti DL, Krapcho $\mathrm{M}$, et al. Collaborative Modeling of the Benefits and Harms Associated With Different U.S. Breast Cancer Screening Strategies. Ann Intern Med. 2016;164(4):215-25.

224. von Fournier D, Weber E, Hoeffken W, Bauer M, Kubli F, Barth V. Growth rate of 147 mammary carcinomas. Cancer. 1980;45(8):2198-207.

225. Sickles EA. Findings at mammographic screening on only one standard projection: outcomes analysis. Radiology. 1998;208(2):471-5.

226. BreastScreen Norway Quality manual .Updated 2019. Available from: https://www.kreftregisteret.no/Generelt/Rapporter/Mammografiprogrammet/Kvalitet/.

227. Leung JW, Sickles EA. Developing asymmetry identified on mammography: correlation with imaging outcome and pathologic findings. AJR Am J Roentgenol. 2007;188(3):667-75.

228. Brennan PC, Ganesan A, Eckstein MP, Ekpo EU, Tapia K, Mello-Thoms C, et al. Benefits of Independent Double Reading in Digital Mammography: A Theoretical Evaluation of All Possible Pairing Methodologies. Acad Radiol. 2019;26(6):717-23.

229. Mullooly M, White G, Bennett K, O'Doherty A, Flanagan F, Healy O. Retrospective radiological review and classification of interval breast cancers within population-based 
breast screening programmes for the purposes of open disclosure: A systematic review. Eur J Radiol. 2021;138:109572.

230. Rothman K. J. Epidemiology an introduction: Oxford University Press; 2012.

231. Bhargava S, Akslen LA, Bukholm IRK, Hofvind S. Performance measures among non-immigrants and immigrants attending BreastScreen Norway: a population-based screening programme. Eur Radiol. 2019;29(9):4833-42.

232. Bhargava S, Tsuruda K, Moen K, Bukholm I, Hofvind S. Lower attendance rates in immigrant versus non-immigrant women in the Norwegian Breast Cancer Screening Programme. J Med Screen. 2018;25(3):155-61.

233. Larsen M, Moshina N, Sagstad S, Hofvind S. Facors associated with attendance and attendance patterns in a population-based mammographic screening program. J Med Screen. 2020:0() 1-8.

234. Laake B, Benestad HB, Olsen BR. Research in Medical and Biological Sciences: Academic Press; 2015.

235. Guidance on applying Duty of Candour and disclosing audit results.: PHE publications gateway number: 2016343; 2016.

236. Expert Reference Group Interval Cancer Report Breast Check. National Screening Service; 2020.

237. Ganz PA, Desmond KA, Leedham B, Rowland JH, Meyerowitz BE, Belin TR. Quality of life in long-term, disease-free survivors of breast cancer: a follow-up study. J Natl Cancer Inst. 2002;94(1):39-49.

238. Ganz PA, Kwan L, Stanton AL, Krupnick JL, Rowland JH, Meyerowitz BE, et al. Quality of life at the end of primary treatment of breast cancer: first results from the moving beyond cancer randomized trial. J Natl Cancer Inst. 2004;96(5):376-87.

239. Durand MA, Friedewald SM, Plecha DM, Copit DS, Barke LD, Rose SL, et al. FalseNegative Rates of Breast Cancer Screening with and without Digital Breast Tomosynthesis.

Radiology. 2021;298(2):296-305.

240. Boyd NF, Huszti E, Melnichouk O, Martin LJ, Hislop G, Chiarelli A, et al. Mammographic features associated with interval breast cancers in screening programs. Breast Cancer Res. 2014;16(4):417.

241. Taplin SH, Rutter CM, Finder C, Mandelson MT, Houn F, White E. Screening mammography: clinical image quality and the risk of interval breast cancer. AJR Am J Roentgenol. 2002;178(4):797-803.

242. Warren LM, Given-Wilson RM, Wallis MG, Cooke J, Halling-Brown MD, Mackenzie A, et al. The effect of image processing on the detection of cancers in digital mammography. AJR Am J Roentgenol. 2014;203(2):387-93.

243. Rodriguez-Ruiz A, Lang K, Gubern-Merida A, Broeders M, Gennaro G, Clauser P, et al. Stand-Alone Artificial Intelligence for Breast Cancer Detection in Mammography:

Comparison With 101 Radiologists. J Natl Cancer Inst. 2019;111(9):916-22.

244. Lang K, Hofvind S, Rodriguez-Ruiz A, Andersson I. Can artificial intelligence reduce the interval cancer rate in mammography screening? Eur Radiol. 2021. 
8. Papers in the thesis with supplementary material 


Paper 3 



\title{
Radiological review of prior screening mammograms of screen-detected breast cancer
}

\author{
Tone Hovda $^{1,2}$ (D) Kaitlyn Tsuruda ${ }^{3,4}$ (D) $\cdot$ Solveig Roth Hoff ${ }^{5,6}$ (D) Kristine Kleivi Sahlberg $^{7,8}$ (D) Solveig Hofvind ${ }^{3,9}$ (D)
}

Received: 23 March 2020 / Revised: 28 May 2020 / Accepted: 31 July 2020 / Published online: 1 October 2020

(C) The Author(s) 2020

\begin{abstract}
Objective To perform a radiological review of mammograms from prior screening and diagnosis of screen-detected breast cancer in BreastScreen Norway, a population-based screening program.

Methods We performed a consensus-based informed review of mammograms from prior screening and diagnosis for screendetected breast cancers. Mammographic density and findings on screening and diagnostic mammograms were classified according to the Breast Imaging-Reporting and Data System ${ }^{\circledR}$. Cases were classified based on visible findings on prior screening mammograms as true (no findings), missed (obvious findings), minimal signs (minor/non-specific findings), or occult (no findings at diagnosis). Histopathologic tumor characteristics were extracted from the Cancer Registry of Norway. The Bonferroni correction was used to adjust for multiple testing; $p<0.001$ was considered statistically significant.

Results The study included mammograms for 1225 women with screen-detected breast cancer. Mean age was 62 years \pm 5 (SD); $46 \%(567 / 1225)$ were classified as true, $22 \%(266 / 1225)$ as missed, and 32\% (392/1225) as minimal signs. No difference in mammographic density was observed between the classification categories. At diagnosis, 59\% (336/567) of true and 70\% (185/ $266)$ of missed cancers were classified as masses $(p=0.004)$. The percentage of histological grade 3 cancers was higher for true $(30 \%(138 / 469))$ than for missed $(14 \%(33 / 234))$ cancers $(p<0.001)$. Estrogen receptor positivity was observed in 86\% $(387 /$ $469)$ of true and $95 \%(215 / 234)$ of missed $(p<0.001)$ cancers.

Conclusions We classified $22 \%$ of the screen-detected cancers as missed based on a review of prior screening mammograms with diagnostic images available. One main goal of the study was quality improvement of radiologists' performance and the program. Visible findings on prior screening mammograms were not necessarily indicative of screening failure.

Key Points

- After a consensus-based informed review, $46 \%$ of screen-detected breast cancers were classified as true, 22\% as missed, and $32 \%$ as minimal signs.

- Less favorable prognostic and predictive tumor characteristics were observed in true screen-detected breast cancer compared with missed.

- The most frequent mammographic finding for all classification categories at the time of diagnosis was mass, while the most frequent mammographic finding on prior screening mammograms was a mass for missed cancers and asymmetry for minimal signs.
\end{abstract}

Keywords Mass screening $\cdot$ Breast neoplasm $\cdot$ Digital mammography $\cdot$ Mammography $\cdot$ Female

Solveig Hofvind

solveig.hofvind@kreftregisteret.no

1 Department of Radiology, Vestre Viken Hospital Trust, PO Box 800, 3004 Drammen, Norway

2 Institute of Clinical Medicine, University of Oslo, PO Box 1171, Blindern, 0318 Oslo, Norway

3 Section for Breast Cancer Screening, Cancer Registry of Norway, PO Box 5313, Majorstuen, 0304 Oslo, Norway

4 Oslo Centre for Biostatistics and Epidemiology, Department of Biostatistics, Institute of Basic Medical Sciences, University of Oslo, PO Box 1122, Blindern, 0317 Oslo, Norway
5 Department of Radiology, Ålesund Hospital, Møre og Romsdal Hospital Trust, Åsehaugen 5, 6017 Ålesund, Norway

6 Department of Circulation and Medical Imaging, Faculty of Medicine and Health Sciences, NTNU, Trondheim, Norway

7 Department of Research and Innovation, Vestre Viken Hospital Trust, PO Box 800, 3004 Drammen, Norway

8 Department of Cancer Genetics, Institute for Cancer Research, Oslo University Hospital Trust, PO Box 4950, 0424 Oslo, Norway

9 Faculty of Health Science, Oslo Metropolitan University, PO Box 4, St. Olavs Plass, 0130 Oslo, Norway 


$\begin{array}{ll}\text { Abbreviations } \\ \text { BI-RADS } & \text { Breast Imaging-Reporting and Data System } \\ \text { CI } & \text { Confidence interval } \\ \text { CRN } & \text { Cancer Registry of Norway } \\ \text { DCIS } & \text { Ductal carcinoma in situ } \\ \text { DM } & \text { Digital mammography } \\ \text { ER } & \text { Estrogen receptor } \\ \text { HER-2 } & \text { Human epidermal growth factor receptor } 2 \\ \text { ILC } & \text { Invasive lobular carcinoma } \\ \text { IQR } & \text { Interquartile range } \\ \text { NST } & \text { No special type } \\ \text { PACS } & \text { Picture archiving and communication system } \\ \text { PR } & \text { Progesterone receptor } \\ \text { SD } & \text { Standard deviation }\end{array}$

\section{Introduction}

Breast cancer is diagnosed among screening participants as screen-detected breast cancer or interval breast cancer (breast cancer diagnosed between two scheduled screening rounds after a negative screening episode). When obvious mammographic findings corresponding to the location of the tumor are visible on prior screening mammograms, which in retrospect should have resulted in a recall, the cancer may be defined as missed. In studies, up to $50 \%$ of interval and screen-detected breast cancers can present visible findings on prior screening mammograms, ranging from minor benign-looking findings to obviously missed cancers [1-8]. Breast cancer can be missed at screening due to misperception - the lesion is not perceived by the radiologist — or misinterpretation - the lesion is detected by the radiologist, but not considered suspicious enough to warrant a recall, either by the reading radiologist or at a consensus meeting. Further, unsatisfactory image quality, positioning, or inadequate assessment at recall may cause a cancer to be missed $[9,10]$. Mammographic density may also impact the rates of missed breast cancer due to the masking effect of dense breast tissue and overlapping structures [11].

Radiologic reviews may be useful for quality assurance and quality improvement of both the program and the radiologists. European guidelines [12] and BreastScreen Norway quality manual [13] recommend continuous surveillance and regular review of screening mammograms performed prior to diagnosis of interval breast cancer. Further, in the National Health Service Breast Screening Programme (UK), radiologists are obliged to audit mammograms of interval breast cancer [14, 15]. Use of audits is discussed also in other countries, and we expect this topic to receive more attention in the future [16]. However, the information available as well as the number of reviewers affect the results of the review [3, 4]. Further, whether a commitment to inform the women will affect the results of a review or an audit is debatable.
Several review studies of interval breast cancer have been performed [8, 17-20]. Larger review studies of screendetected breast cancer, particularly including digital mammography (DM), are, to our knowledge, sparse. Screendetected breast cancer with no visible findings on prior screening mammograms, defined as true cases, may grow faster than missed breast cancer. Thus, different histopathological characteristics and different mammographic findings are anticipated for true versus missed screen-detected breast cancers.

We conducted a nationwide consensus-based, informed review within BreastScreen Norway. The study included prior screening mammograms and mammograms available at diagnosis from women diagnosed with screen-detected breast cancer. The overall aim of the study was quality improvement for radiologists' performance and the program as such. The objectives were to investigate the proportions of true and missed screen-detected breast cancers and to explore whether mammographic findings, density, or histopathological characteristics differed between the two groups. We hypothesized that these three aspects differed between true and missed screendetected breast cancers.

\section{Materials and methods}

The study was approved by the data protection official at the Cancer Registry of Norway (CRN) (PVO approval number: 2016/4696), and the local breast centers agreed to the study. The Cancer Registry Regulation waived the requirement to obtain written informed consent for use of screening data for quality assurance and research [21]. We received de-identified data for analyses from the CRN.

BreastScreen Norway offers women aged 50-69 biennial screening with two-view standard DM. The screening exams take place at 27 stationary or mobile units. Screen reading is performed at 16 breast centers and includes independent double reading by breast radiologists. The radiologists score each breast on a 5-point scale; 1 indicates negative findings, and 5 indicates a high suspicion for malignancy. Exams scored $\geq 2$ by either radiologist are discussed in a consensus meeting to decide whether to recall the woman [22]. The median annual reading volume for radiologists during 1996-2016 was 4492 exams; $46 \%$ of the radiologists had over 10 years of screen reading experience [23].

\section{Materials and review logistics}

CRN randomly extracted 85 screen-detected breast cancer cases from each of the 16 breast centers. All examinations were performed with DM during 2006-2015, and all women had a screening exam with DM 2 years previously (prior screen). We aimed to review at least 75 cases at each center within an 8-h session, including instruction time. Panels of 5 
breast radiologists performed the review from September 2016 to April 2017. Radiologists not participating in the panel could observe the review session.

We performed a consensus-based, fully informed retrospective radiological review. All screening and diagnostic images were available to the reviewers, including ultrasound and MRI, as well as histopathological reports. The breast centers were divided into 8 pairs; two radiologists from each center reviewed each other's images and made up the consensus panel together with one independent radiologist, the first author (T.H.). To qualify for the panel, we required the radiologists to have at least 1 year of experience in screen reading and a reading volume of $\geq 5000$ mammograms during the past 2 years.

To ensure consistency across the centers in the review procedures, classifications, registration, and coding of results, T.H. took part in all reviews together with a representative from CRN. Ahead of each session, T.H. presented the classification systems and general instructions for the review. In the case of dissent among the panel members, a majority decision was made. All images were reviewed locally from the picture archiving and communication system (PACS).

\section{Review procedure}

The review procedure is described in Fig. 1. First, we reviewed the mammograms, resulting in recall and diagnosis of screen-detected breast cancer, and thereafter classified mammographic density using the Breast Imaging-Reporting and Data System (BI-RADS) 5th edition categories a-d [24]. We identified the malignancy and classified mammographic findings as mass, calcifications, asymmetry, distortion, or associated findings using the BI-RADS lexicon. If calcifications were present alongside another finding, the non-calcification finding was preferred for classification, unless calcifications were the dominant finding. We classified the largest tumor in case of multifocality or bilateral disease. We measured the diameter $(\mathrm{mm})$ of the findings on the mammogram, using electronic calipers. If no malignancy was visible at the time of diagnosis, the case was classified as occult.

Thereafter, we reviewed and classified the finding, based on its visibility on prior screening mammograms. True cancers showed no findings at the eventual cancer site on prior screening mammograms (Fig. 2a, b). Cancers with obvious findings at the cancer site on priors which retrospectively should have resulted in a recall, as considered by the reviewing radiologists, were defined as missed (Fig. 2c, d). Minimal sign cancers showed minor findings on prior mammograms, not necessarily warranting assessment (Fig. 2e, f). At review, minimal signs were classified as either actionable (recall considered possible, but not expected within a screening program) or non-actionable (non-specific findings, recall not considered possible). However, we consider all minimal signs as one category in the main analyses. Finally, we classified mammographic findings on prior mammograms for missed and minimal sign cancers.

Histopathological information extracted from the $\mathrm{CRN}$ database was merged with data from the review and communicated to the radiologists after complete classification of each case. Prognostic characteristics included histological type (ductal carcinoma in situ (DCIS), invasive carcinoma of no special type (NST), invasive lobular carcinoma (ILC), other invasive carcinomas) and, for invasive cancers, also histological grade, histopathological tumor diameter ( $\mathrm{mm}$ ), and lymph node status. Predictive tumor characteristics for invasive cancers included estrogen receptor (ER) and progesterone receptor (PR) status.

\section{Statistical analyses}

We performed descriptive analyses of age at diagnosis, review classification categories, mammographic findings, mammographic density, and histopathological characteristics. Data were presented as percentages with $95 \%$ confidence intervals (CIs), calculated using the Clopper-Pearson mid-P interval; means \pm standard deviations (SDs); and medians with interquartile ranges (IQRs). Chi-square tests and independent sample $t$ tests were used to test the differences between review classification categories and mammographic findings or histopathological characteristics, as well as between mammographic findings and histopathological characteristics. The Bonferroni correction was used to adjust for multiple testing, and a $p$ value $<0.001$ was considered statistically significant. IBM SPSS Statistics (version 25) was used for all analyses.

\section{Results}

We reviewed and classified mammograms from 1227 women screened with DM, recalled due to mammographic findings and diagnosed with breast cancer. We excluded two mammographically occult cases. The final study sample thus consisted of 1225 cases.

Mean age at diagnosis was $62 \pm 5$ years, and median age was 63 years $(59,66)$. We classified $46 \%(567 / 1225)$ of the screen-detected cancers as true, $22 \%(266 / 1225)$ as missed, and $32 \%(392 / 1225)$ as minimal signs.

At the time of diagnosis, 59\% (336/567) of true, 70\% (185/ $266)$ of missed, and $65 \%$ (256/392) of minimal signs were masses $(p=0.004$ for true versus missed), while 23\% (128/ $567)$ of true, $17 \%(46 / 266)$ of missed, and 17\% (68/392) of minimal signs were calcifications. The mean diameter of mammographic findings at diagnosis was $18 \mathrm{~mm} \pm 14$ for true, $20 \mathrm{~mm} \pm 15$ for missed, and $17 \mathrm{~mm} \pm 16$ for minimal signs (Table 1). 
Fig. 1 Review procedure

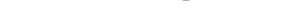

\begin{tabular}{|c|c|c|c|}
\hline \multicolumn{4}{|c|}{ REVIEW OF MAMMOGRAMS AT TIME OF DIAGNOSIS } \\
\hline \multicolumn{4}{|c|}{ Classification of mammographic density (BI-RADS a-d) } \\
\hline \multicolumn{4}{|c|}{ Breast cancer visible on mammograms at time of diagnosis? } \\
\hline \multicolumn{3}{|c|}{ YES } & NO \\
\hline $\begin{array}{l}\text { Mammographic fin } \\
\text { Mass } \\
\text { Calcifications } \\
\text { Asymmetry }\end{array}$ & $\begin{array}{l}\text { Dgs at diagnosis } \\
\text { Distortion } \\
\text { Associated findi } \\
\text { Diameter of finc }\end{array}$ & $\mathrm{gs}(\mathrm{mm})$ & \\
\hline \multicolumn{3}{|c|}{ REVIEW OF PRIOR SCREENING MAMMOGRAMS } & \\
\hline $\begin{array}{l}\text { No findings at the } \\
\text { later cancer site }\end{array}$ & $\begin{array}{c}\text { Obvious findings at } \\
\text { the later cancer } \\
\text { site }\end{array}$ & $\begin{array}{l}\text { Minor or non-specific } \\
\text { findings at the later } \\
\text { cancer site }\end{array}$ & \\
\hline True & Missed & $\begin{array}{l}\text { Minimal signs } \\
\text { actionable/non } \\
\text { actionable }\end{array}$ & Occult \\
\hline & \multicolumn{2}{|c|}{ 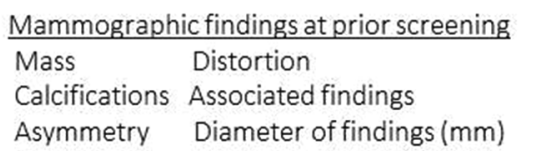 } & \\
\hline \multicolumn{4}{|c|}{ HISTOPATHOLOGICAL REPORTS } \\
\hline $\begin{array}{l}\text { Prognostic factors } \\
\text { Tumor diameter } \\
\text { Histopathological } \\
\text { Lymph node status }\end{array}$ & pe and grade & $\begin{array}{l}\text { Predictive factors } \\
\text { ER-status } \\
\text { PR-status }\end{array}$ & \\
\hline
\end{tabular}
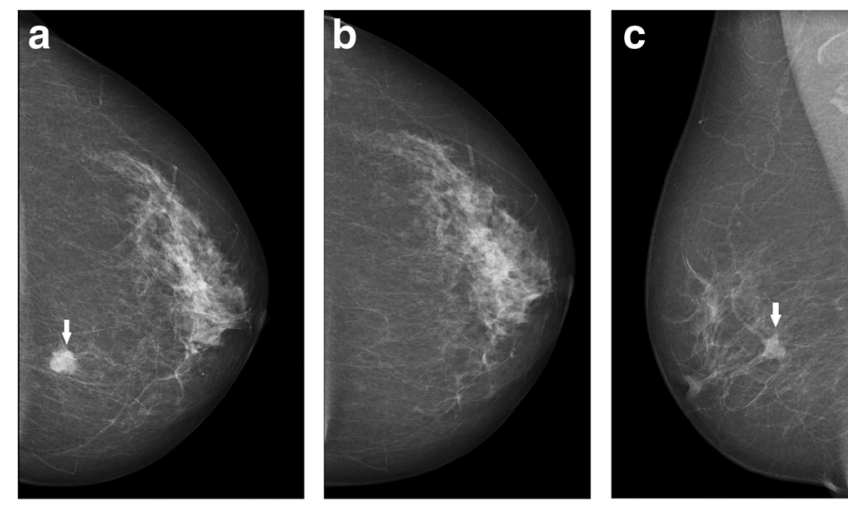

Fig. 2 a, b True screen-detected breast cancer. A 57-year-old woman presenting with an irregular mass in the medial aspect of the left breast at screening (arrow), diagnosed with a 17-mm invasive carcinoma of no special type. Left craniocaudal view at diagnosis (a) and at prior screening (b). c, d Missed screen-detected breast cancer. A 67-year-old woman presenting with a spiculated mass in the upper lateral part of the right breast at screening (arrow), diagnosed with an 18-mm invasive lobular
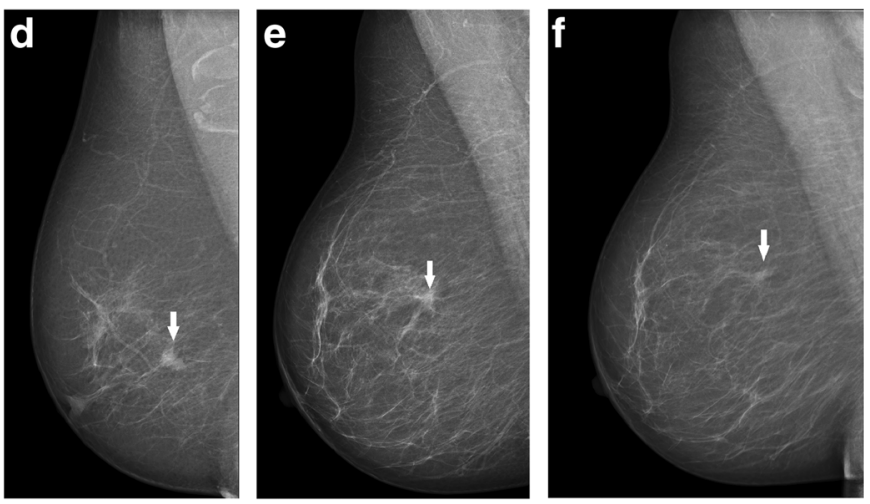

carcinoma. Right mediolateral oblique (MLO) view at diagnosis (c). A spiculated mass at the later cancer site (arrow) also appears at prior screening (d). e, f Minimal sign screen-detected breast cancer. A 67year-old woman presenting with a spiculated mass in the upper outer quadrant at screening (arrow), right MLO view (e), diagnosed with a 17-mm invasive lobular carcinoma. A non-specific focal asymmetry (arrow) is visible on prior screening mammograms (f) 
Table 1 Mammographic findings at diagnosis and on prior screening mammograms stratified by review classification categories

\begin{tabular}{|c|c|c|c|c|c|c|c|c|}
\hline & \multicolumn{2}{|l|}{ Total } & \multicolumn{2}{|l|}{ True } & \multicolumn{2}{|l|}{ Missed } & \multicolumn{2}{|c|}{ Minimal signs } \\
\hline & $n=1225$ & $100 \%$ & $n=567$ & $46 \%$ & $n=266$ & $22 \%$ & $n=392$ & $32 \%$ \\
\hline \multicolumn{9}{|l|}{ Age (years) } \\
\hline Mean \pm SD & & 62 years \pm 5 & & 62 years \pm 5 & & 63 years \pm 5 & & 62 years \pm 5 \\
\hline Median (IQR) & & 63 years $(59,66)$ & & 63 years $(58,66)$ & & 64 years $(60,67)$ & & 63 years $(59,66)$ \\
\hline \multicolumn{9}{|c|}{ Mammographic density } \\
\hline BI-RADS $a+b$ & 810 & $66 \%(63,69)$ & 372 & $66 \%(62,70)$ & 178 & $67 \%(61,73)$ & 260 & $66 \%(61,71)$ \\
\hline BI-RADS c + d & 415 & $34 \%(31,37)$ & 195 & $34 \%(31,39)$ & 88 & $33 \%(28,39)$ & 132 & $34 \%(29,39)$ \\
\hline \multicolumn{9}{|c|}{ Mammographic findings at diagnosis } \\
\hline Mass & 777 & $63 \%(61,66)$ & 336 & $59 \%(55,63)$ & 185 & $70 \%(66,75)$ & 256 & $65 \%(60,70)$ \\
\hline Calcifications & 242 & $20 \%(18,22)$ & 128 & $23 \%(19,26)$ & 46 & $17 \%(13,22)$ & 68 & $17 \%(14,22)$ \\
\hline Asymmetry & 106 & $9 \%(7,10)$ & 58 & $10 \%(8,13)$ & 15 & $6 \%(3,9)$ & 33 & $8 \%(6,12)$ \\
\hline Distortion & 97 & $8 \%(7,10)$ & 43 & $8 \%(6,10)$ & 20 & $8 \%(5,11)$ & 34 & $9 \%(6,12)$ \\
\hline Associated findings & 3 & $0.2 \%(0,1)$ & 2 & $0.4 \%(0,1)$ & & & 1 & $0.3 \%(0,1)$ \\
\hline \multicolumn{9}{|c|}{ Mammographic diameter } \\
\hline Mean \pm SD & \multicolumn{2}{|c|}{$18 \mathrm{~mm} \pm 15$} & \multicolumn{2}{|c|}{$18 \mathrm{~mm} \pm 14$} & \multicolumn{2}{|c|}{$20 \mathrm{~mm} \pm 15$} & \multicolumn{2}{|c|}{$17 \mathrm{~mm} \pm 16$} \\
\hline Median (IQR) & \multicolumn{2}{|c|}{$14 \mathrm{~mm}(10,21)$} & \multicolumn{2}{|c|}{$14 \mathrm{~mm}(10,20)$} & \multicolumn{2}{|c|}{$16 \mathrm{~mm}(11,23)$} & \multicolumn{2}{|c|}{$14 \mathrm{~mm}(10,21)$} \\
\hline Data not available & \multicolumn{2}{|l|}{83} & \multicolumn{2}{|l|}{46} & \multicolumn{2}{|l|}{5} & \multicolumn{2}{|l|}{30} \\
\hline \multicolumn{9}{|c|}{ Mammographic findings prior mammograms } \\
\hline Mass & 134 & $20 \%(17,24)$ & & & 115 & $43 \%(37,49)$ & 19 & $5 \%(3,8)^{*}$ \\
\hline Calcifications & 142 & $22 \%(19,25)$ & & & 62 & $23 \%(18,29)$ & 80 & $20 \%(17,25)$ \\
\hline Asymmetry & 302 & $46 \%(42,50)$ & & & 66 & $25 \%(20,31)$ & 236 & $60 \%(55,65)^{*}$ \\
\hline Distortion & 78 & $12 \%(10,15)$ & & & 23 & $9 \%(6,13)$ & 55 & $14 \%(11,18)$ \\
\hline Associated findings & 2 & $0.3 \%(0,1)$ & & & & & 2 & $0.5 \%(0,2)$ \\
\hline \multicolumn{9}{|c|}{ Mammographic diameter } \\
\hline Mean \pm SD & \multicolumn{2}{|c|}{$12 \mathrm{~mm} \pm 11$} & & & \multicolumn{2}{|c|}{$14 \mathrm{~mm} \pm 12$} & \multicolumn{2}{|c|}{$10 \mathrm{~mm} \pm 9^{*}$} \\
\hline Median (IQR) & \multicolumn{2}{|c|}{$9 \mathrm{~mm}(6,14)$} & & & \multicolumn{2}{|c|}{$11 \mathrm{~mm}(7,15)$} & \multicolumn{2}{|c|}{$8 \mathrm{~mm}(5,12)$} \\
\hline Data not available & & 191 & & & & 20 & & 171 \\
\hline
\end{tabular}

Unless otherwise specified, data are the number of patients and $95 \%$ confidence intervals in parenthesis

$S D$ standard deviation, $I Q R$ interquartile range

$* p<0.001$, compared with missed

At prior screening, 43\% (115/266) of missed cancers and 5\% $(19 / 392)$ of minimal signs were masses $(p<0.001) ; 25 \%(66 /$ $266)$ of missed and $60 \%$ (236/392) of minimal signs presented as asymmetries $(p<0.001)$. The mean diameter of mammographic findings on prior screening mammograms was $14 \mathrm{~mm} \pm 12$ for missed and $10 \mathrm{~mm} \pm 9$ for minimal signs $(p<0.001)$ (Table 1$)$.

Eighty-two percent (247/302) of cases classified as asymmetries on prior mammograms were classified as masses at diagnosis. Ninety-nine percent $(132 / 134)$ of the cases classified as masses at priors were classified as masses at diagnosis; $79 \%(112 / 142)$ of calcifications on priors also presented as such at diagnosis, and $18 \%$ (26/142) of calcifications presented as a mass. Among cancers classified as distortions on priors, $51 \%(40 / 78)$ were classified as distortions at diagnosis and $46 \%(36 / 78)$ as a mass (Fig. 3$)$.

We did not observe any differences in mammographic density between classification groups; the percentage of BI-
RADS a + b was $66 \%$ (372/567) for true, $67 \%$ (178/266) for missed, and 66\% (260/392) for minimal signs (Table 1, Fig. 4a). However, the percentages of calcifications and distortions were statistically higher in mammograms classified with high (BI-RADS c $+d$ ) mammographic density compared with low (BI-RADS $a+b)$, both at diagnosis (Fig. 4b) and at prior screening (Fig. 4c).

No statistically significant differences were observed in the distribution of histopathological type for missed, true, or minimal signs; DCIS accounted for $17 \%(98 / 567)$ of the true, $12 \%$ $(32 / 266)$ of the missed, and $13 \%(50 / 392)$ of the minimal signs (Table 2).

Among invasive cancers, 30\% (138/457) of true cancers were histological grade 3, compared with $14 \%$ (33/230) of missed and $15 \%(51 / 342)$ of minimal signs $(p<0.001$ for missed or minimal signs versus true). We observed no differences in mean histopathologic tumor diameter or lymph node 
Fig. 3 Mammographic findings on prior screening mammograms of missed and minimal sign cancers, stratified by mammographic findings on mammograms at diagnosis of screen-detected breast cancer

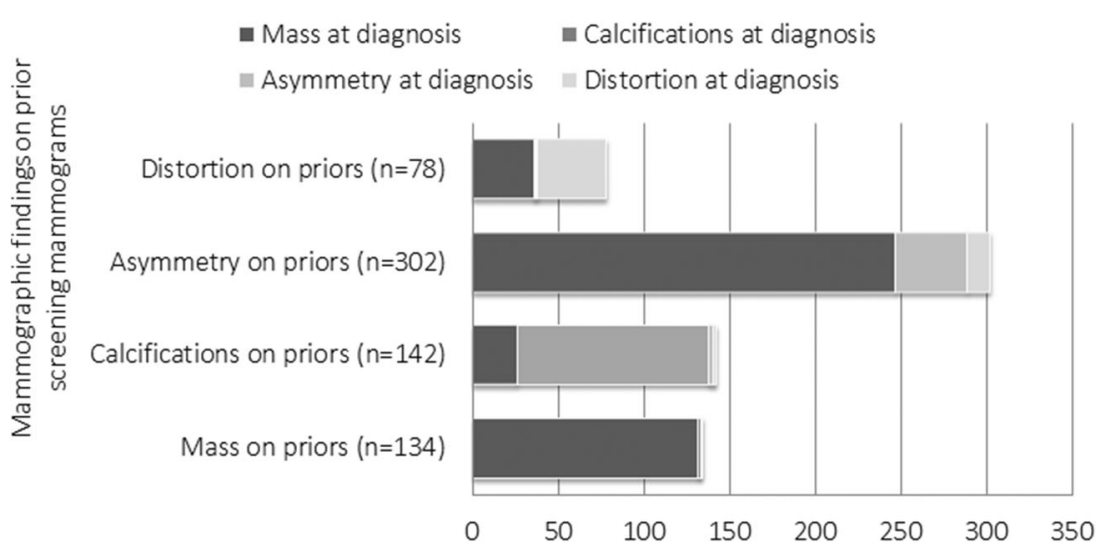

status between the groups. The percentage of ER+ cases was $86 \%(387 / 451)$ for true and $95 \%$ for both missed $(215 / 226)$ and minimal signs $(317 / 333)(p<0.001$ for missed or minimal signs versus true). Sixty-seven percent (303/453) of true, $78 \%$ (173/223) of missed, and 77\% (254/330) of minimal sign cancers were $\mathrm{PR}+(p=0.004$ for missed versus true and $p=0.002$ for minimal signs versus true) (Table 2).

We observed the highest percentage of DCIS, $60 \%$ (145/ 242) among cases presenting as calcifications (Table 3). Masses, asymmetries, and distortions were mainly invasive carcinoma of NST, ranging from 71\% (69/97) for distortions to $83 \%(646 / 777)$ for masses. We observed $21 \%$ (20/97) for ILC among distortions, compared with 7\% (54/777) for masses and 2\% (5/242) for calcifications. Among invasive cancers, distortions were associated with the lowest percentage of histological grade 3 tumors $(8 \%, 7 / 92)$ and calcifications were associated with the highest $(41 \%, 39 / 94)$. The mean mammographic diameter for invasive cancers ranged from $15 \mathrm{~mm} \pm 8$ (masses) to $36 \mathrm{~mm} \pm 26$ (calcifications). Mean histopathologic tumor diameter ranged from $12 \mathrm{~mm} \pm$ 11 (calcifications) to $20 \mathrm{~mm} \pm 15$ (distortions).

For masses, the percentage of histological grade 3 invasive cancer was higher for true than for missed and minimal sign screen-detected breast cancer $(p<0.001$ for true compared with both missed and minimal signs); otherwise, we observed no differences in histopathological tumor characteristics stratified by review classification categories and mammographic findings (Table 4). We observed no differences for mammographic findings, histologic tumor type, diameter, and grade for minimal signs, actionable versus non-actionable tumors (Table 5 in the Appendix).

\section{Discussion}

In this informed, consensus-based review of mammograms from prior screening and diagnosis of 1225 women with screen-detected cancer, radiologists classified $46 \%$ as true, $22 \%$ as missed, and $32 \%$ as minimal signs. The most frequent mammographic finding at diagnosis was a mass for all classification categories; no statistically significant differences were observed between the classification categories regarding mammographic findings at diagnosis. At prior screening, the most frequent mammographic finding for missed cancer was a mass, whereas for minimal sign cancer, it was asymmetry. The majority of asymmetries at prior screening progressed
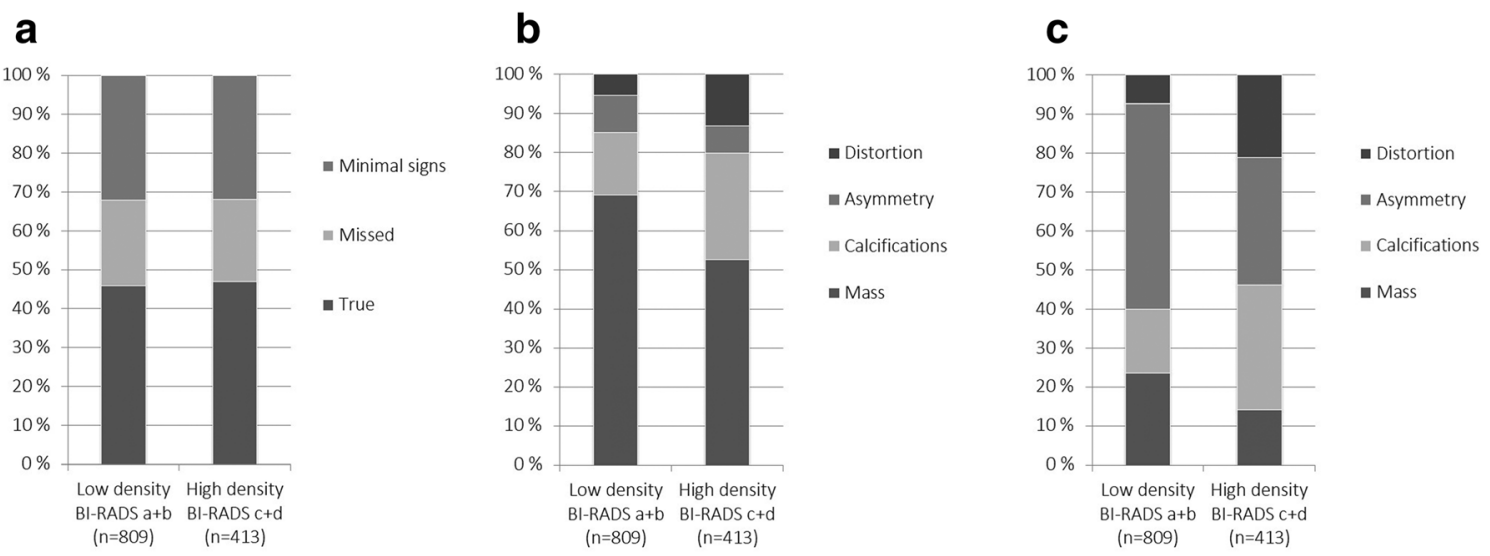

Fig. 4 a Distribution of review classification groups based on findings on prior screening mammograms (true, missed, or minimal signs) stratified by the BI-RADS density score (low: BI-RADS a + b; high: BI-RADS c + d) $(p=0.88)$. $\mathbf{b}$ Distribution of mammographic findings at diagnosis

(mass, calcifications, asymmetry, or distortion) stratified by the BIRADS density score $(p<0.001)$. c Distribution of mammographic findings on prior screening mammograms stratified by the BI-RADS density score $p<0.001$ 
Table 2 Histopathological tumor characteristics stratified by review classification categories

\begin{tabular}{|c|c|c|c|c|c|c|c|c|}
\hline & \multicolumn{2}{|l|}{ Total } & \multicolumn{2}{|c|}{ True } & \multicolumn{2}{|c|}{ Missed } & \multicolumn{2}{|c|}{ Minimal signs } \\
\hline & $n=1225$ & $100 \%$ & \multicolumn{2}{|c|}{$n=567$} & \multicolumn{2}{|c|}{$n=266$} & \multicolumn{2}{|c|}{$n=392$} \\
\hline \multicolumn{9}{|l|}{ Histopathological type } \\
\hline DCIS & 180 & $15 \%(13,17)$ & 98 & $17 \%(14,21)$ & 32 & $12 \%(8,17)$ & 50 & $13 \%(10,17)$ \\
\hline Invasive carcinoma of NST & 880 & $72 \%(69,74)$ & 407 & $72 \%(68,76)$ & 194 & $73 \%(67,78)$ & 279 & $71 \%(66,76)$ \\
\hline ILC & 94 & $8 \%(6,9)$ & 35 & $6 \%(4,9)$ & 21 & $8 \%(5,12)$ & 38 & $10 \%(7,13)$ \\
\hline Other invasive carcinomas & 71 & $6 \%(5,7)$ & 27 & $5 \%(3,7)$ & 19 & $7 \%(4,11)$ & 25 & $6 \%(4,9)$ \\
\hline DCIS & 180 & & 98 & & 32 & & 50 & \\
\hline \multicolumn{9}{|l|}{ Tumor diameter } \\
\hline Mean \pm SD & \multicolumn{2}{|c|}{$20 \mathrm{~mm} \pm 16$} & \multicolumn{2}{|c|}{$20 \mathrm{~mm} \pm 14$} & \multicolumn{2}{|c|}{$18 \mathrm{~mm} \pm 14$} & \multicolumn{2}{|c|}{$23 \mathrm{~mm} \pm 21$} \\
\hline Median (IQR) & \multicolumn{2}{|c|}{$15 \mathrm{~mm}(10,27)$} & \multicolumn{2}{|c|}{$15 \mathrm{~mm}(10,26)$} & \multicolumn{2}{|c|}{$15 \mathrm{~mm}(8,25)$} & \multicolumn{2}{|c|}{$17 \mathrm{~mm}(10,33)$} \\
\hline Data not available & \multicolumn{2}{|l|}{23} & \multicolumn{2}{|l|}{15} & \multicolumn{2}{|l|}{2} & \multicolumn{2}{|l|}{6} \\
\hline \multicolumn{9}{|l|}{ Histological grade } \\
\hline Grade 1 & 23 & $14 \%(9,21)$ & 9 & $10 \%(5,19)$ & 3 & $10 \%(2,27)$ & 11 & $24 \%(13,39)$ \\
\hline Grade 2 & 18 & $11 \%(7,17)$ & 10 & $12 \%(6,20)$ & 3 & $10 \%(2,27)$ & 5 & $11 \%(4,24)$ \\
\hline Grade 3 & 121 & $75 \%(67,81)$ & 68 & $78 \%(68,86)$ & 23 & $79 \%(60,92)$ & 30 & $65 \%(50,79)$ \\
\hline Data not available & 18 & & 11 & & 3 & & 4 & \\
\hline Invasive cancer & 1045 & & 469 & & 234 & & 342 & \\
\hline \multicolumn{9}{|l|}{ Tumor diameter (mm) } \\
\hline Mean \pm SD & \multicolumn{2}{|c|}{$15 \mathrm{~mm} \pm 10$} & \multicolumn{2}{|c|}{$15 \mathrm{~mm} \pm 9$} & \multicolumn{2}{|c|}{$16 \mathrm{~mm} \pm 10$} & \multicolumn{2}{|c|}{$15 \mathrm{~mm} \pm 10$} \\
\hline Median (IQR) & $13 \mathrm{~mm}$ & , 19) & 12 & $9,18)$ & 13 & $10,20)$ & 13 & , 19) \\
\hline Data not available & 23 & & 12 & & 5 & & 6 & \\
\hline Histological grade & & & & & & & & \\
\hline Grade 1 & 288 & $28 \%(25,30)$ & 103 & $23 \%(19,27)$ & 73 & $32 \%(26,38)$ & 112 & $33 \%(28,38)$ \\
\hline Grade 2 & 519 & $50 \%(47,54)$ & 216 & $47 \%(43,52)$ & 124 & $54 \%(47,61)$ & 179 & $52 \%(47,58)$ \\
\hline Grade 3 & 222 & $22 \%(19,24)$ & 138 & $30 \%(26,35)$ & 33 & $14 \%(10,20)^{*}$ & 51 & $15 \%(11,19)^{*}$ \\
\hline Data not available & 16 & & 12 & & 4 & & & \\
\hline Lymph node status & & & & & & & & \\
\hline Negative & 797 & $80 \%(77,82)$ & 353 & $78 \%(74,82)$ & 182 & $81 \%(76,86)$ & 262 & $81 \%(76,85)$ \\
\hline Data not available & 47 & & 19 & & 10 & & 18 & \\
\hline Hormonal status & & & & & & & & \\
\hline ER positive & 919 & $91 \%(89,93)$ & 387 & $86 \%(82,89)$ & 215 & $95 \%(92,98)^{*}$ & 317 & $95 \%(92,97)^{*}$ \\
\hline Data not available & 35 & & 18 & & 8 & & 9 & \\
\hline PR positive & 730 & $73 \%(70,75)$ & 303 & $67 \%(62,71)$ & 173 & $78 \%(72,83)$ & 254 & $77 \%(72,81)$ \\
\hline Data not available & 39 & & 16 & & 11 & & 12 & \\
\hline
\end{tabular}

Unless otherwise specified, data are the number of patients and $95 \%$ confidence intervals in parentheses

$D C I S$ ductal carcinoma in situ, NST no special type, ILC invasive lobular carcinoma, ER estrogen receptor, $P R$ progesterone receptor, SD standard deviation, $I Q R$ interquartile range

$* p<0.001$, compared with true

into masses by the time of diagnosis. Mammographic density did not differ between the review classification categories. True invasive cancers were more often histological grade 3 and had less favorable hormonal status than missed and minimal sign invasive cancers.

Our findings support results from other retrospective, informed review studies of screen-detected breast cancer. In a study by Ikeda et al [8], findings at the later cancer site, obvious or non-specific, were observed retrospectively in $67 \%$ of screen-detected breast cancers. Van Breest Smallenburg et al [9] found $21 \%$ of screen-detected breast cancer to be missed and $22 \%$ with non-specific minimal signs at informed review, and Broeders et al [7] identified findings on prior screening mammograms in $53 \%$ of screen-detected cancers. However, 
Table 3 Histopathological tumor characteristics stratified by mammographic findings

\begin{tabular}{|c|c|c|c|c|c|c|c|c|}
\hline & \multicolumn{2}{|l|}{ Mass } & \multicolumn{2}{|c|}{ Calcifications } & \multicolumn{2}{|c|}{ Asymmetry } & \multicolumn{2}{|c|}{ Distortion } \\
\hline & \multicolumn{2}{|c|}{$n=777$} & \multicolumn{2}{|c|}{$n=242$} & \multicolumn{2}{|c|}{$n=106$} & \multicolumn{2}{|c|}{$n=97$} \\
\hline \multicolumn{9}{|l|}{ Histopathological type } \\
\hline DCIS & 23 & $3 \%(2,4) *$ & 145 & $60 \%(53,66)$ & 9 & $9 \%(4,16)^{*}$ & 3 & $3 \%(1,9)^{*}$ \\
\hline Invasive carcinoma of NST & 646 & $83 \%(80,86)^{*}$ & 85 & $35 \%(29,42)$ & 78 & $74 \%(64,82)^{*}$ & 69 & $71 \%(61,80)^{*}$ \\
\hline ILC & 54 & $7 \%(5,9)^{* *}$ & 5 & $2 \%(1,5)$ & 14 & $13 \%(7,21)^{*}$ & 20 & $21 \%(13,30)^{*}$ \\
\hline Other invasive carcinomas & 54 & $7 \%(5,9)$ & 7 & $3 \%(1,6)$ & 5 & $5 \%(2,11)$ & 5 & $5 \%(2,12)$ \\
\hline Invasive cancer & 754 & & 97 & & 97 & & 94 & \\
\hline \multicolumn{9}{|l|}{ Histological grade } \\
\hline Grade 1 & 211 & $28 \%(25,32)$ & 13 & $14 \%(8,23)$ & 26 & $28 \%(1,38)$ & 38 & $41 \%(31,52)^{*}$ \\
\hline Grade 2 & 378 & $51 \%(47,54)$ & 42 & $45 \%(34,55)$ & 51 & $54 \%(44,65)$ & 47 & $51 \%(40,62)$ \\
\hline Grade 3 & 158 & $21 \%(18,24)^{*}$ & 39 & $41 \%(31,52)$ & 17 & $18 \%(11,27)^{*}$ & 7 & $8 \%(3,15)^{*}$ \\
\hline Data not available & 7 & & 3 & & 3 & & 2 & \\
\hline \multicolumn{9}{|l|}{ Lymph node status } \\
\hline Negative & 583 & $80 \%(77,83)$ & 73 & $85 \%(76,92)$ & 74 & $80 \%(70,87)$ & 67 & $74 \%(64,83)$ \\
\hline Data not available & 28 & 28 & 11 & & 4 & & 4 & \\
\hline \multicolumn{9}{|l|}{ Hormonal status } \\
\hline ER positive & 661 & $90 \%(88,92)$ & 75 & $85 \%(76,92)$ & 89 & $95 \%(88,98)$ & 92 & $100 \%(96,100)^{*}$ \\
\hline Data not available & 21 & & 9 & & 3 & & 2 & \\
\hline PR positive & 532 & $73 \%(70,76)^{*}$ & 47 & $54 \%(43,65)$ & 69 & $73 \%(63,82)$ & 80 & $87 \%(78,93)^{*}$ \\
\hline Data not available & 24 & & 10 & & 3 & & 2 & \\
\hline \multicolumn{9}{|l|}{ Mammographic diameter (mm) } \\
\hline Mean \pm SD & & $15 \mathrm{~mm} \pm 8^{*}, * *$ & & $36 \mathrm{~mm} \pm 26$ & & $17 \mathrm{~mm} \pm 16^{*}$ & & $22 \mathrm{~mm} \pm 15^{*}$ \\
\hline Median (IQR) & & $13 \mathrm{~mm}(9,18)$ & & $30 \mathrm{~mm}(17,50)$ & & $13 \mathrm{~mm}(9,20)$ & & $20 \mathrm{~mm}(14,25)$ \\
\hline Data not available & 45 & & 7 & & 7 & & 4 & \\
\hline \multicolumn{9}{|l|}{ Tumor diameter (mm) } \\
\hline Mean \pm SD & & $15 \mathrm{~mm} \pm 8$ & & $12 \mathrm{~mm} \pm 11$ & & $16 \mathrm{~mm} \pm 12$ & & $20 \mathrm{~mm} \pm 15^{*}$ \\
\hline Median (IQR) & & $12 \mathrm{~mm}(9,18)$ & & $9 \mathrm{~mm}(5,17)$ & & $14 \mathrm{~mm}(8,17)$ & & $15 \mathrm{~mm}(12,22)$ \\
\hline Data not available & 11 & & 9 & & 0 & & 2 & \\
\hline
\end{tabular}

Unless otherwise specified, data are the number of patients and $95 \%$ confidence interval in parentheses

$D C I S$ ductal carcinoma in situ, NST no special type, ILC invasive lobular carcinoma, $E R$ estrogen receptor, $P R$ progesterone receptor, $S D$ standard deviation, IQR interquartile range

$* p<0.001$, compared with calcifications; $* * p<0.001$, compared with distortion

all three studies included screen-film mammography (SFM). In an informed review from BreastScreen Norway, $12 \%$ of screen-detected breast cancers were classified as missed and 9\% minimal sign actionable for screening with SFM [6] and $10 \%$ missed and $9 \%$ minimal sign actionable for screening with DM [1]. However, the review procedures and definition of classification groups differed between the studies, making comparison challenging. In experimental review studies of interval breast cancer exploring different study designs, the percentage of cancers classified as missed differed largely depending on review procedure and number of radiologists. Hofvind et al [3] found the percentage of missed interval breast cancer ranging from $1 \%$ (mixed, blinded individual review) to 34\% (informed, consensus-based) and 36\% (informed individual). In a study by Ciatto et al [4], the proportion of missed (screening error) cancers varied from $24 \%$ in a simulated blinded review to $42 \%$ in a simulated fully informed review. Further, studies have demonstrated that the proportion of missed cancer is affected by how close the study setting is to a normal screening setting $[25,26]$. Easy understandable standardized definitions and recommendations on classification groups and review procedure are needed to enable future comparison of results from reviews.

True cancers include tumors detected at an early stage, reflected by the higher percentage of DCIS among true versus missed cancers. However, true cancers may also be fast- 
Table 4 Histopathological type, tumor diameter, and histological grade for mammographic findings stratified by review categories

\begin{tabular}{|c|c|c|c|c|c|c|}
\hline & True & & Missed & & Minimal signs & \\
\hline Mass & $n=336$ & & $n=185$ & & $n=256$ & \\
\hline \multicolumn{7}{|l|}{ Histopathological type } \\
\hline DCIS & 9 & $3 \%(1,5)$ & 7 & $4 \%(2,8)$ & 7 & $3 \%(1,6)$ \\
\hline Invasive carcinoma of NST & 291 & $87 \%(83,90)$ & 149 & $81 \%(74,86)$ & 206 & $81 \%(75,85)$ \\
\hline ILC & 17 & $5 \%(3,8)$ & 11 & $6 \%(3,10)$ & 26 & $10 \%(7,15)$ \\
\hline Other invasive carcinomas & 19 & $6 \%(3,9)$ & 18 & $10 \%(6,15)$ & 17 & $7 \%(4,10)$ \\
\hline \multicolumn{7}{|c|}{ Tumor diameter of invasive cancer } \\
\hline Mean \pm SD & $14 \mathrm{~mm} \pm 8$ & & $15 \mathrm{~mm} \pm 9$ & & $14 \mathrm{~mm} \pm 8$ & \\
\hline Median (IQR) & $12 \mathrm{~mm}(9,18)$ & & $13 \mathrm{~mm}(10,18)$ & & $12 \mathrm{~mm}(9,19)$ & \\
\hline Not available & 5 & & 3 & & 3 & \\
\hline \multicolumn{7}{|c|}{ Histological grade of invasive cancer } \\
\hline Grade 1 & 72 & $22 \%(18,27)$ & 61 & $35 \%(28,42)$ & 78 & $31 \%(26,38)$ \\
\hline Grade 2 & 150 & $47 \%(41,52)$ & 96 & $54 \%(47,62)$ & 132 & $53 \%(47,59)$ \\
\hline Grade 3 & 99 & $31 \%(26,36)$ & 20 & $11 \%(7,17)^{*}$ & 39 & $16 \%(11,21)^{*}$ \\
\hline Not available & 6 & & 1 & & 0 & \\
\hline Asymmetry & $n=58$ & & $n=15$ & & $n=33$ & \\
\hline \multicolumn{7}{|l|}{ Histopathological type } \\
\hline DCIS & 5 & $9 \%(3,19)$ & & & 4 & $12 \%(3,28)$ \\
\hline Invasive carcinoma of NST & 46 & $79 \%(67,89)$ & 10 & $67 \%(38,88)$ & 22 & $67 \%(48,82)$ \\
\hline ILC & 4 & $7 \%(2,17)$ & 15 & $33 \%(12,62)$ & 5 & $15 \%(5,32)$ \\
\hline Other invasive carcinomas & 3 & $5 \%(1,14)$ & & & 2 & $6 \%(1,20)$ \\
\hline \multicolumn{7}{|c|}{ Tumor diameter (mm) of invasive cancer } \\
\hline Mean \pm SD & \multicolumn{2}{|l|}{$14 \mathrm{~mm} \pm 11$} & \multicolumn{2}{|l|}{$20 \mathrm{~mm} \pm 9$} & \multicolumn{2}{|l|}{$16 \mathrm{~mm} \pm 14$} \\
\hline Median (IQR) & \multicolumn{2}{|l|}{$13 \mathrm{~mm}(8,16)$} & \multicolumn{2}{|l|}{$19 \mathrm{~mm}(15,24)$} & \multicolumn{2}{|l|}{$14 \mathrm{~mm}(8,16)$} \\
\hline \multicolumn{7}{|l|}{ Not available } \\
\hline \multicolumn{7}{|c|}{ Histological grade of invasive cancer } \\
\hline Grade 1 & 10 & $20 \%(10,34)$ & 2 & $13 \%(2,41)$ & 14 & $48 \%(29,68)$ \\
\hline Grade 2 & 26 & $52 \%(37,66)$ & 10 & $67 \%(38,88)$ & 15 & $52 \%(33,71)$ \\
\hline Grade 3 & 14 & $28 \%(16,43)$ & 3 & $20 \%(4,48)$ & & \\
\hline Not available & 3 & & & & & \\
\hline Calcifications & $n=128$ & & $n=46$ & & $n=68$ & \\
\hline \multicolumn{7}{|l|}{ Histopathological type } \\
\hline DCIS & 83 & $65 \%(56,73)$ & 23 & $50 \%(35,65)$ & 39 & $57 \%(45,69)$ \\
\hline Invasive carcinoma of NST & 39 & $31 \%(23,39)$ & 21 & $46 \%(31,61)$ & 25 & $37 \%(25,49)$ \\
\hline ILC & 3 & $2 \%(1,7)$ & 1 & $2 \%(0,12)$ & 1 & $2 \%(0,8)$ \\
\hline Other invasive carcinomas & 3 & $2 \%(1,7)$ & 1 & $2 \%(0,12)$ & 3 & $4 \%(1,12)$ \\
\hline \multicolumn{7}{|c|}{ Tumor diameter of invasive cancer } \\
\hline Mean \pm SD & $13 \mathrm{~mm} \pm 12$ & & $11 \mathrm{~mm} \pm 10$ & & $12 \mathrm{~mm} \pm 9$ & \\
\hline Median (IQR) & $10 \mathrm{~mm}(6,15)$ & & $6 \mathrm{~mm}(4,11)$ & & $10 \mathrm{~mm}(5,19)$ & \\
\hline Not available & 5 & & 2 & & 2 & \\
\hline Histological grade of invasive & & & & & & \\
\hline Grade 1 & 6 & $14 \%(5,27)$ & 3 & $14 \%(3,36)$ & 4 & $14 \%(4,32)$ \\
\hline Grade 2 & 18 & $41 \%(26,57)$ & 10 & $48 \%(26,70)$ & 14 & $48 \%(29,68)$ \\
\hline Grade 3 & 20 & $45 \%(30,61)$ & 8 & $38 \%(18,62)$ & 11 & $38 \%(21,58)$ \\
\hline Not available & 1 & & 2 & & & \\
\hline Distortion & $n=43$ & & $n=20$ & & $n=34$ & \\
\hline Histopathological type & & & & & & \\
\hline DCIS & 1 & $2 \%(0,12)$ & 2 & $10 \%(1,32)$ & & \\
\hline Invasive carcinoma of NST & 29 & $67 \%(52,81)$ & 14 & $70 \%(46,88)$ & 26 & $77 \%(59,89)$ \\
\hline ILC & 11 & $26 \%(14,41)$ & 4 & $20 \%(6,44)$ & 5 & $15 \%(5,31)$ \\
\hline Other invasive carcinomas & 2 & $5 \%(1,16)$ & & & 3 & $9 \%(2,24)$ \\
\hline Tumor diameter (mm) of inva & & & & & & \\
\hline Mean \pm SD & $19 \mathrm{~mm} \pm 13$ & & $21 \mathrm{~mm} \pm 14$ & & $20 \mathrm{~mm} \pm 17$ & \\
\hline Median (IQR) & $16 \mathrm{~mm}(10,22)$ & & $17 \mathrm{~mm}(12,22)$ & & $15 \mathrm{~mm}(12,22)$ & \\
\hline Not available & 1 & & & & 1 & \\
\hline Histological grade of invasive & & & & & & \\
\hline Grade 1 & 15 & $37 \%(2,53)$ & 7 & $41 \%(18,67)$ & 16 & $47 \%(30,65)$ \\
\hline Grade 2 & 22 & $54 \%(37,69)$ & 8 & $47 \%(23,72)$ & 17 & $50 \%(32,68)$ \\
\hline Grade 3 & 4 & $10 \%(3,23)$ & 2 & $12 \%(2,36)$ & 1 & $3 \%(0,15)$ \\
\hline Not available & 1 & & 1 & & & \\
\hline
\end{tabular}

Unless otherwise specified, data are the number of patients and $95 \%$ confidence intervals in parentheses

$D C I S$ ductal carcinoma in situ, NST no special type, ILC invasive lobular carcinoma, ER estrogen receptor, $P R$ progesterone receptor, $S D$ standard deviation, $I Q R$ interquartile range

$* p<0.001$, compared with true. 
growing tumors, with less favorable tumor characteristics [27, 28]. This is illustrated in our study by the larger percentage of histological grade 3 invasive cancer among true compared with missed and minimal sign cancers. The largest discrepancy in the diameter of mammographic findings versus histopathological tumor diameter was observed among calcifications $(36 \mathrm{~mm} \pm 26$ versus $12 \mathrm{~mm} \pm 11)$. Invasive cancers presenting as calcifications were also smaller and had a higher histological grade than cancers presenting with other mammographic findings. This may reflect small, aggressive invasive tumors imbedded in a larger calcified area, possibly of DCIS. However, we cannot elaborate on this hypothesis because only the diameter of the invasive component is coded in the database at the CRN. The larger tumor diameter and higher percentage of tumors with low/intermediate histological grade among distortions may be related to the increased frequency of ILC among distortions. ILC is generally of low/ intermediate histological grade and larger at diagnosis than other invasive carcinomas [29-31].

A mass was the most frequent finding on prior mammograms of missed cancers, and a special emphasis on masses at screening might be reasonable. Masses might be misinterpreted as benign, in particular if retrospectively visible on more than one prior screening exam or if not having spiculated margins $[18,32]$. Further, the mean diameter of mammographic findings of missed cancer was $11 \mathrm{~mm}$, which usually is regarded to be above the limit for visual perception. This could indicate that a certain proportion of cancers was missed due to misinterpretation at screen reading or dismissed at consensus. The high frequency of asymmetries on priors of missed and minimal signs developing into masses at the screening exam leading to diagnosis of cancer is in line with other studies; increased awareness of asymmetries may be useful to reduce the burden of missed cancers at screening $[32,33]$. However, asymmetries are common and most often represent glandular tissue, in particular if visible only in one view. Thus, radiologists should be attentive and should avoid an unreasonably increase in the recall rate for such findings. Evaluating more than one prior screening exam may be valuable in this respect. Moreover, a recent study showed that increasing the recall rate mainly increased detection of low-grade and not high-grade cancer [34]. This is consistent with our results demonstrating a higher proportion of tumors of low and intermediate histological grade among missed/minimal signs than true; an increased recall rate would probably reduce the proportion of missed and minimal signs.

Our study has some limitations. First, the review was consensus-based and all images were available - this design yields the highest percentage of missed cancers in review studies [3-5]. This limits the external generalizability of our results. Second, our study was performed at 16 breast centers with images from a span of approximately 10 years, and major heterogeneity in the combinations of PACS, workstations, and mammographic equipment. As a result, the image quality and presentation differed between centers, which might have influenced the assessment of review classifications, mammographic findings, and density. Third, the consensus panel included five radiologists: one who participated in all the reviews and four who only participated in one session at their own and one at the paired center. Although we presented and communicated the general instructions and classification systems to all radiologists at the start of each review, some differences in interpretation and assessment between radiologists are likely to have occurred. However, we included mammograms and radiologists from all breast centers in Norway, and our study is as far as we are aware of, the largest reported in peer-reviewed journals, which we consider as strength. Finally, we assessed mammographic density from the screening mammograms at diagnosis. This might have biased the association between review categories (classified from prior screening mammograms 2 years earlier) and mammographic density, as the women's breast density might have decreased during the 2 years.

The study confirmed our hypothesis that mammographic findings and histopathologic characteristics differed between true and missed screen-detected breast cancers in BreastScreen Norway. However, we did not identify differences in mammographic density between review classification categories. One main goal of the classification of missed cancers was quality improvement for radiologists' performance and the program. We would like to emphasize that the review and study setting differed substantially from real-life screening settings. Visible findings on priors were not necessarily indicative of a screening failure. Recalling all women with subtle findings would increase the rate of false positive recalls and probably also the detection of small, low proliferation tumors (overdiagnosis). This is important to keep in mind during audits and when exploring medicolegal aspects of mammographic screening.

Acknowledgments Vestre Viken Hospital Trust funded the salary for TH during the project period. SH is the head of BreastScreen Norway.

Funding Open Access funding provided by University of Oslo (incl Oslo University Hospital).

\section{Compliance with ethical standards}

Guarantor The scientific guarantor of this publication is Prof. Solveig Hofvind.

Conflict of interest The authors of this manuscript declare no relationships with any companies whose products or services may be related to the subject matter of the article.

Statistics and biometry One of the authors (K. Tsuruda) has significant statistical expertise.

Informed consent Written informed consent was waived by the institutional review board. The Cancer Registry Regulation gives approval with 
waiver of informed consent to perform surveillance, quality assurance, and studies based on data collected as a part of invitation to and participation in the program

Ethical approval Institutional review board approval was obtained. Data was collected from BreastScreen Norway and thus covered by the Cancer Registry Regulation. Data was approved by the institutional Data Protection Officer at the Cancer Registry and the Heads of Department and/or research administration at the local breast centers.
Study subjects or cohorts overlap Some study subjects or cohorts are reported in the study of Tsuruda K, et al: Survival among women diagnosed with breast cancer retrospectively classified as true, missed, or minimal signs through radiological review. Will be submitted to European Radiology, March 2020.

\section{Methodology}

- retrospective

- observational

- multicenter study

\section{Appendix}

Table 5 Mammographic findings and histopathological characteristics for minimal signs actionable versus minimal signs non-actionable

\begin{tabular}{|c|c|c|c|c|}
\hline & \multicolumn{2}{|c|}{ Minimal signs actionable } & \multicolumn{2}{|c|}{ Minimal signs non-actionable } \\
\hline & $n=192$ & & $n=200$ & \\
\hline \multicolumn{5}{|l|}{ Diagnostic mammograms } \\
\hline Mass & 140 & $73 \%(66,79)^{*}$ & 116 & $58 \%(51,65)$ \\
\hline Calcifications & 24 & $13 \%(8,18)$ & 44 & $22 \%(17,28)$ \\
\hline Asymmetry & 13 & $7 \%(4,11)$ & 20 & $10 \%(6,15)$ \\
\hline Distortion & 15 & $8 \%(4,13)$ & 19 & $10 \%(6,14)$ \\
\hline Associated features & & & 1 & $0.5 \%(0,3)$ \\
\hline \multicolumn{5}{|l|}{ Mammographic diameter } \\
\hline Mean \pm SD & & $20 \mathrm{~mm} \pm 17$ & & $18 \mathrm{~mm} \pm 15$ \\
\hline Median (IQR) & & $15 \mathrm{~mm}(10,22)$ & & $14 \mathrm{~mm}(9,20)$ \\
\hline Data not available & 14 & & 16 & \\
\hline \multicolumn{5}{|l|}{ Prior mammograms } \\
\hline Mass & 13 & $7 \%(4,11)$ & 6 & $3 \%(1,6)$ \\
\hline Calcifications & 32 & $17 \%(12,23)$ & 48 & $24 \%(18,31)$ \\
\hline Asymmetry & 117 & $61 \%(54,68)$ & 119 & $60 \%(52,66)$ \\
\hline Distortion & 29 & $15 \%(1021)$ & 26 & $13 \%(9,19)$ \\
\hline Associated features & 1 & $0.5 \%(0,3)$ & 1 & $0.5 \%(0,3)$ \\
\hline \multicolumn{5}{|l|}{ Histopathological type } \\
\hline DCIS & 20 & $10 \%(7,16)$ & 30 & $15 \%(10,21)$ \\
\hline Invasive carcinoma of NST & 138 & $72 \%(65,78)$ & 141 & $71 \%(64,77)$ \\
\hline ILC & 19 & $10 \%(6,15)$ & 19 & $10 \%(6,14)$ \\
\hline Other & 15 & $8 \%(4,13)$ & 10 & $5 \%(2,9)$ \\
\hline Invasive cancer & $\mathrm{n}=172$ & & $\mathrm{n}=170$ & \\
\hline \multicolumn{5}{|l|}{ Tumor diameter } \\
\hline Mean \pm SD & & $16 \mathrm{~mm} \pm 11$ & & $14 \mathrm{~mm} \pm 9$ \\
\hline Median (IQR) & & $13 \mathrm{~mm}(9,19)$ & & $13 \mathrm{~mm}(8,20)$ \\
\hline Data not available & 4 & & 2 & \\
\hline \multicolumn{5}{|l|}{ Histological grade } \\
\hline Grade 1 & 54 & $31 \%(25,39)$ & 58 & $34 \%(27,42)$ \\
\hline Grade 2 & 96 & $56 \%(48,63)$ & 83 & $49 \%(41,57)$ \\
\hline Grade 3 & 22 & $13 \%(8,19)$ & 29 & $17 \%(12,24)$ \\
\hline
\end{tabular}

Unless otherwise specified, data are the numbers of patient and $95 \%$ confidence intervals in parentheses

$D C I S$ ductal carcinoma in situ, NST no special type, ILC invasive lobular carcinoma, SD standard deviation, IQR interquartile range $* p=0.002$, compared with minimal sign actionable 
Open Access This article is licensed under a Creative Commons Attribution 4.0 International License, which permits use, sharing, adaptation, distribution and reproduction in any medium or format, as long as you give appropriate credit to the original author(s) and the source, provide a link to the Creative Commons licence, and indicate if changes were made. The images or other third party material in this article are included in the article's Creative Commons licence, unless indicated otherwise in a credit line to the material. If material is not included in the article's Creative Commons licence and your intended use is not permitted by statutory regulation or exceeds the permitted use, you will need to obtain permission directly from the copyright holder. To view a copy of this licence, visit http://creativecommons.org/licenses/by/4.0/.

\section{References}

1. Hoff SR, Abrahamsen AL, Samset JH, Vigeland E, Klepp O, Hofvind S (2012) Breast cancer: missed interval and screening-detected cancer at full-field digital mammography and screen-film mammographyresults from a retrospective review. Radiology 264:378-386

2. Moberg K, Grundstrom H, Lundquist H, Svane G, Havervall E, Muren C (2000) Radiological review of incidence breast cancers. J Med Screen 7:177-183

3. Hofvind S, Skaane P, Vitak B et al (2005) Influence of review design on percentages of missed interval breast cancers: retrospective study of interval cancers in a population-based screening program. Radiology 237:437-443

4. Ciatto S, Catarzi S, Lamberini MP et al (2007) Interval breast cancers in screening: the effect of mammography review method on classification. Breast 16:646-652

5. Houssami N, Hunter K (2017) The epidemiology, radiology and biological characteristics of interval breast cancers in population mammography screening. NPJ Breast Cancer 3:12

6. Hoff SR, Samset JH, Abrahamsen AL, Vigeland E, Klepp O, Hofvind S (2011) Missed and true interval and screen-detected breast cancers in a population based screening program. Acad Radiol 18:454-460

7. Broeders MJ, Onland-Moret NC, Rijken HJ, Hendriks JH, Verbeek AL, Holland R (2003) Use of previous screening mammograms to identify features indicating cases that would have a possible gain in prognosis following earlier detection. Eur J Cancer 39:1770-1775

8. Ikeda DM, Birdwell RL, O'Shaughnessy KF, Brenner RJ, Sickles EA (2003) Analysis of 172 subtle findings on prior normal mammograms in women with breast cancer detected at follow-up screening. Radiology 226:494-503

9. van Breest SV, Setz-Pels W, Groenewoud JH et al (2012) Malpractice claims following screening mammography in the Netherlands. Int J Cancer 131:1360-1366

10. Pinto A, Acampora C, Pinto F, Kourdioukova E, Romano L, Verstraete K (2011) Learning from diagnostic errors: a good way to improve education in radiology. Eur J Radiol 78:372-376

11. Shawky MS, Huo CW, Henderson MA, Redfern A, Britt K, Thompson EW (2019) A review of the influence of mammographic density on breast cancer clinical and pathological phenotype. Breast Cancer Res Treat 177:251-276

12. Perry N,Broeders M de Wolf C, Tornberg S, Holland R, von Karsa L(2006) European guidelines for quality assurance in breast cancer screening and diagnosis. Brussels, Belgium: European Communities

13. Quality manual BreastScreen Norway, Cancer Registry of Norway. Available via https://www.kreftregisteret.no/Generelt/Rapporter/ Mammografiprogrammet/Kvalitet/. Accessed Dec 2019

14. NHS Breast Screening Programme (2017) Reporting, classification and monitoring of interval cancers and cancers following previous assessment. Public Health England. Available via https://www.gov.uk/government/ publications/breast-screening-interval-cancers. Accessed Dec 2019
15. Lekanidi K, Dilks P, Suaris T, Kennett S, Purushothaman H (2017) Breast screening: What can the interval cancer review teach us? Are we perhaps being a bit too hard on ourselves? Eur J Radiol 94:13-15

16. Geertse TD, Holland R, Timmers JM et al (2015) Value of audits in breast cancer screening quality assurance programmes. Eur Radiol 25:3338-3347

17. Bansal GJ, Thomas KG (2011) Screen-detected breast cancer: does presence of minimal signs on prior mammograms predict staging or grading of cancer? Clin Radiol 66:605-608

18. Roberts-Klein S, Iuanow E, Slanetz PJ (2011) Avoiding pitfalls in mammographic interpretation. Can Assoc Radiol J 62:50-59

19. Bare M, Tora N, Salas D et al (2015) Mammographic and clinical characteristics of different phenotypes of screen-detected and interval breast cancers in a nationwide screening program. Breast Cancer Res Treat 154:403-415

20. Domingo L, Romero A, Blanch J et al (2013) Clinical and radiological features of breast tumors according to history of false-positive results in mammography screening. Cancer Epidemiol 37:660-665

21. Ministry of Health and Care Services (2001) Forskrift om innsamling og behandling av helseopplysninger i Kreftregisteret (The Cancer Registry Regulation).

22. Hofvind S, Tsuruda K, Mangerud $G$ et al (2017) The Norwegian Breast Cancer Screening Program, 1996-2016: celebrating 20 years of organised mammographic screening cancer in Norway 2016 - cancer incidence, mortality, survival and prevalence in Norway. Cancer Registry of Norway

23. Hoff SR, Myklebust TA, Lee CI, Hofvind S (2019) Influence of mammography volume on radiologists' performance: results from BreastScreen Norway. Radiology 292:289-296

24. Sickles E, D’Orsi CJ, Bassett LW et al (2013) ACR BI-RADS® mammography. In: ACR BI-RADS® atlas, Breast Imaging Reporting and Data System. American College of Radiology, Reston

25. Moberg K, Grundstrom H, Tornberg S et al (1999) Two models for radiological reviewing of interval cancers. J Med Screen 6:35-39

26. Gordon PB, Borugian MJ, Warren Burhenne LJ (2007) A true screening environment for review of interval breast cancers: pilot study to reduce bias. Radiology 245:411-415

27. Domingo L, Salas D, Zubizarreta R et al (2014) Tumor phenotype and breast density in distinct categories of interval cancer: results of population-based mammography screening in Spain. Breast Cancer Res 16:R3

28. Hofvind S, Sagstad S, Sebuodegard S, Chen Y, Roman M, Lee CI (2018) Interval breast cancer rates and histopathologic tumor characteristics after false-positive findings at mammography in a population-based screening program. Radiology 287:58-67

29. Chamming's F, Kao E, Aldis A et al (2017) Imaging features and conspicuity of invasive lobular carcinomas on digital breast tomosynthesis. Br J Radiol 90:20170128

30. Chen Z, Yang J, Li S et al (2017) Invasive lobular carcinoma of the breast: a special histological type compared with invasive ductal carcinoma. PLoS One 12:e182397

31. Li CI, Uribe DJ, Daling JR (2005) Clinical characteristics of different histologic types of breast cancer. Br J Cancer 93:1046-1052

32. Wadhwa A, Sullivan JR, Gonyo MB (2016) Missed breast cancer: what can we learn? Curr Probl Diagn Radiol 45:402-419

33. Chesebro AL, Winkler NS, Birdwell RL, Giess CS (2016) Developing asymmetries at mammography: a multimodality approach to assessment and management. Radiographics 36:322-334

34. Blanks RG, Given-Wilson RM, Cohen SL, Patnick J, Alison RJ, Wallis MG (2019) An analysis of 11.3 million screening tests examining the association between recall and cancer detection rates in the English NHS breast cancer screening programme. Eur Radiol 29:3812-3819

Publisher's note Springer Nature remains neutral with regard to jurisdictional claims in published maps and institutional affiliations. 
Paper 4 



\title{
True and Missed Interval Cancer in Organized Mammographic Screening: A Retrospective Review Study of Diagnostic and Prior Screening Mammograms
}

\author{
Tone Hovda, MD, Solveig Roth Hoff, MD, PhD, Marthe Larsen, MSc, Linda Romundstad, MD,
} Kristine Kleivi Sahlberg, MSc, PhD, Solveig Hofvind, PhD

\begin{abstract}
Rationale and Objectives: To explore radiological aspects of interval breast cancer in a population-based screening program.
Materials and Methods: We performed a consensus-based informed review of mammograms from diagnosis and prior screening from women diagnosed with interval cancer 2004-2016 in BreastScreen Norway. Cases were classified as true (no findings on prior screening mammograms), occult (no findings at screening or diagnosis), minimal signs (minor/non-specific findings) and missed (obvious findings). We analyzed mammographic findings, density, time since prior screening, and histopathological characteristics between the classification groups.

Results: The study included 1010 interval cancer cases. Mean age at diagnosis was 61 years (SD =6), mean time between screening and diagnosis 14 months $(S D=7)$. A total of $48 \%(479 / 1010)$ were classified as true or occult, $28 \%(285 / 1010)$ as minimal signs and $24 \%$ (246/1010) as missed. We observed no differences in mammographic density between the groups, except from a higher percentage of dense breasts in women with occult cancer. Among cancers classified as missed, about $1 / 3$ were masses and $1 / 3$ asymmetries at prior screening. True interval cancers were diagnosed later in the screening interval than the other classification categories. No differences in histopathological characteristics were observed between true, minimal signs and missed cases.
\end{abstract}

Conclusion: In an informed review, $24 \%$ of the interval cancers were classified as missed based on visibility and mammographic findings on prior screening mammograms. Three out of four true interval cancers were diagnosed in the second year of the screening interval. We observed no statistical differences in histopathological characteristics between true and missed interval cancers.

Key Words: Mass screening; Breast neoplasm; Digital mammography; Mammograph; Female.

(C) 2021 The Association of University Radiologists. Published by Elsevier Inc. All rights reserved.

Abbreviations: BI-RADS Breast Imaging Reporting and Data System, CI Confidence interval, DBT Digital breast tomosynthesis, DM Standard Digital mammography, IQR Interquartile range, NST Invasive carcinoma no special type, PACS Picture archiving and communication system, SD Standard deviation

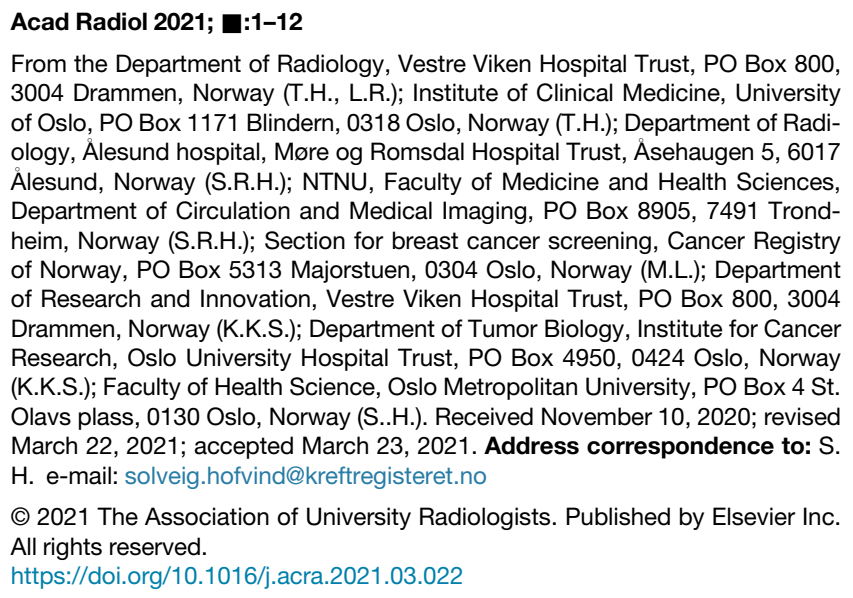

From the Department of Radiology, Vestre Viken Hospital Trust, PO Box 800, 3004 Drammen, Norway (T.H., L.R.); Institute of Clinical Medicine, University of Oslo, PO Box 1171 Blindern, 0318 Oslo, Norway (T.H.); Department of Radiology, Allesund hospital, Møre og Romsdal Hospital Trust, Assehaugen 5, 6017 Ålesund, Norway (S.R.H.); NTNU, Faculty of Medicine and Health Sciences, Department of Circulation and Medical Imaging, PO Box 8905, 7491 Trondheim, Norway (S.R.H.); Section for breast cancer screening, Cancer Registry of Norway, PO Box 5313 Majorstuen, 0304 Oslo, Norway (M.L.); Department of Research and Innovation, Vestre Viken Hospital Trust, PO Box 800, 3004 Drammen, Norway (K.K.S.); Department of Tumor Biology, Institute for Cancer Research, Oslo University Hospital Trust, PO Box 4950, 0424 Oslo, Norway (K.K.S.); Faculty of Health Science, Oslo Metropolitan University, PO Box 4 St. Olavs plass, 0130 Oslo, Norway (S..H.). Received November 10, 2020; revised March 22, 2021; accepted March 23, 2021. Address correspondence to: S. H. e-mail: solveig.hofvind@kreftregisteret.no

(C) 2021 The Association of University Radiologists. Published by Elsevier Inc. All rights reserved.

https://doi.org/10.1016/j.acra.2021.03.022

\section{INTRODUCTION}

nterval breast cancer is diagnosed between two scheduled screening rounds, after a negative screening episode or after a negative recall assessment (1). The causes of interval cancers are heterogeneous; some cancers grow rapidly and become clinically and/or radiologically detectable in the inter-screening interval as true interval cancer, while others have been misinterpreted or missed by radiologists at screen reading or consensus/arbitration (2,3). Mammographically occult cancers are not visible on the mammograms even at the time of diagnosis, usually due to tissue overlap or localization outside the target area of the mammogram. Interval cancers with visible findings on prior screening mammograms in 
hindsight may be classified as minimal signs or missed, depending on the level of suspiciousness of the mammographic findings $(1,2,4)$. Houssami explored several review studies to estimate the proportion of cancers with visible findings on prior screening mammograms (2). However, different study design and classification systems hamper comparison of results and in the reported studies the proportion of missed interval cancer ranged from 13\%-35\%.

Rates of interval cancer vary across screening programs $(10,11)$ and it is estimated that interval cancer accounts for $17 \%-30 \%$ of the cancers in biennial screening programs (2). In general, interval cancers have less favorable histopathological tumor characteristics and reduced survival compared with screen-detected cancers (5-9). Further, the majority of the interval cancers is diagnosed in the second half of the screening interval. In addition to the radiologists' performance, factors influencing the rates and proportions of interval cancer within a program include the background breast cancer incidence, the screening interval, the definition of interval breast cancer and completeness of cancer registration (12). Interval cancer is an inevitable part of all screening programs, but it is important to keep the rates as low as possible, not only to reduce mortality, but also to increase trust in the program among women in the target population, and in the society in general. In BreastScreen Norway, the rate of interval cancer has been relatively stable over the past 20 years. In the period from 1996-2016, the interval cancer rate was 1.9 per 1000 screened women, comprising $24 \%$ of all breast cancers among the participants in the program (13).

To our knowledge, no unanimous results exist regarding mammographic findings of prior screening mammograms for missed and minimal signs interval breast cancer. Further, whether or how mammographic density varies for different classification categories is not fully understood (3,14-16). In programs without a central cancer registry, registration of interval breast cancer may be inadequate, in particular if the interval cancer is diagnosed in another clinic than the screening took place, or the woman has moved to another district (10). The Cancer Registry of Norway and BreastScreen Norway is unique in terms of completeness and organization of screening data. By law, all hospitals, medical laboratories and doctors report all cancer cases to the registry and linked with screening data, information on interval cancers is almost $100 \%$ complete and available (17).

We took advantage of this completeness and performed a fully informed, consensus-based review of diagnostic and prior screening mammograms from women diagnosed with interval cancer in BreastScreen Norway. The overall aim of the study was to explore radiological aspects of the interval cancers. We described the proportions of true, occult, minimal signs and missed interval breast cancer, and analyzed associations between these groups and mammographic findings, time since prior screening, and histopathological characteristics.

\section{MATERIAL AND METHODS}

The Data Protection Officer for the Cancer Registry of Norway and the Heads of Department and/or research administration at the local breast centers approved this retrospective study. The Cancer Registry Regulations waived the requirement to obtain written informed consent (17).

\section{Screening Logistics in BreastScreen Norway}

In BreastScreen Norway, women aged 50-69 are invited biennially to screening with two-view digital mammography (DM) at 27 stationary or mobile units. Screen-reading and recall assessment is performed by breast radiologists at 17 breast centers (the number of breast centers was 16 when the review was performed). The screen-reading includes independent double reading with consensus/arbitration. Two radiologists assign each breast a score of 1-5 $(1=$ normal/benign; 2 = probably benign; $3=$ indeterminate; $4=$ probably malignant; $5=$ malignant) and all exams scored $\geq 2$ by either radiologist are discussed in a consensus meeting to decide whether to recall the woman (13). The median annual reading volume for radiologists in BreastScreen Norway during the period from 1996 to 2016 was 4492 exams, and $46 \%$ of the radiologists had more than 10 years of screen-reading experience (18). Pathological specimens were examined by pathologists at the local hospitals. All screening data from BreastScreen Norway, including results from histopathological reports, are reported to the Cancer Registry of Norway and stored in a national database.

\section{Materials}

We aimed at reviewing prior screening mammograms of 1200 interval cancer cases; 75 cases from each of the 16 breast centers, spending no more than 8 hours for each review session. From the screening database at the Cancer Registry, data on interval cancer cases diagnosed during the period from March 2004 to September 2016 were identified and extracted. We included standard DM performed in the screening round prior to the diagnosis (within 24 months), and diagnostic DM. To take into account that some cases might not have diagnostic images available for review (interval cancer diagnosed at a private clinic or in another hospital), up to 90 cases were identified from each center. However, the aim of 75 reviewed cases was chieved in 3 centers; the remaining centers were either not able to provide as many as 75 interval cancer cases with diagnostic and prior images available (either due to a small center size, or many diagnostic exams performed outside the center), or the review session reached the time limit of 8 hours (Supplementary Table 1). The final study population included 1010 interval cancer cases. For comparative analyses of interval versus screen-detected cancer, we used information from a 
parallel review study of 1257 screen-detected cases. This study is described in detail elsewhere (19).

\section{Review Procedure}

At each breast center, panels of five breast radiologists performed a fully informed consensus-based review of diagnostic and prior screening mammograms of women diagnosed with interval cancer. The review panel consisted of two radiologists employed at the breast center whose images were reviewed, two radiologists who worked at one of the other 15 breast centers, and additionally, T.H. participated in all review sessions. The mammograms were reviewed locally from the picture archiving and communication system. The classifications were all based on majority decisions. The review logistics was similar to a prior review of screen-detected cancer and is described in more detail elsewhere (19).

We identified the tumor on the diagnostic images, reviewed the prior screening mammograms and classified the case according to findings as follows: True (no visible findings on priors), occult (mammographically occult at diagnosis), minimal signs (minor or non-specific findings, recall assessment not mandatory or even not possible within the screening program) or missed (obvious visible abnormal findings on priors) (Fig 1). We classified the mammographic findings on diagnostic and prior screening images according to the American College of Radiology's Breast Imaging - Reporting and Data System (BI-RADS) $5^{\text {th }}$ edition lexicon (20); mass, calcifications, asymmetry, distortion or other findings, with subclassifications when appropriate (Fig 2). We classified the largest tumor in case of multifocal or bilateral disease, and if calcifications were present alongside another finding (mass, asymmetry or distortion), we classified the malignancy as calcifications only if this was the dominant finding. We measured the diameter of the suspicious mammographic findings using an electronic caliper and classified mammographic density on diagnostic mammograms into BI-RADS 5th edition categories a (entirely fatty), $\mathrm{b}$ (scattered areas of fibroglandular density), c (heterogeneously dense) or d (extremely dense).

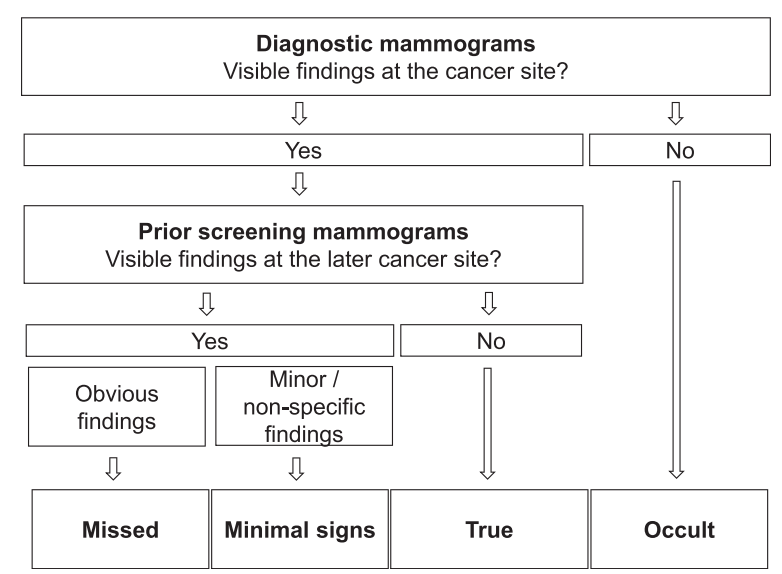

Figure 1. Review procedure.
Histopathological tumor characteristics from the Cancer Registry's database were communicated to the radiologists after complete classification of each case and merged with data from the review. These characteristics included histological type (ductal carcinoma in situ, invasive carcinoma of no special type (NST), invasive lobular carcinoma, other invasive carcinoma), and for invasive cancers Histological Grade 1-3, histopathological tumor diameter $(\mathrm{mm})$, lymph node status (positive/negative), estrogen receptor and progesterone receptor status (positive/negative)

\section{Statistical Analyses}

We performed descriptive analyses of age at diagnosis of interval cancer (years), time between diagnosis and the prior screening exam (months), review classification categories, mammographic findings, mammographic density, and histopathological characteristics. Categorical data were presented as frequencies and percentages. Means and standard deviations (SD) were presented for normally distributed continuous variables (age, time since screening), and medians with interquartile ranges (IQR) were presented for non-normally distributed continuous variables (mammographic and histopathological diameter). We used Chisquare tests or Fisher's exact test when appropriate (categorical data), independent sample t-tests (means) and nonparametric tests (medians) to test for differences between review classification categories and mammographic findings or histopathological characteristics. We used the Bonferroni correction to adjust for multiple testing and considered $p<0.001$ statistically significant. We used IBM SPSS Statistics version 25 for all analyses.

\section{RESULTS}

We classified $35 \%(353 / 1010)$ of the cases as true interval cancer, $13 \%(126 / 1010)$ as occult, 28\% (285/1010) as minimal signs and $24 \%(246 / 1010)$ as missed (Fig 3a-d). Mean age at diagnosis for all women was 61 years $(\mathrm{SD}=6)$. No statistically significant difference in age between the review classification categories was identified (Table 1).

\section{Time From Screening to Diagnosis}

Mean time from screening to diagnosis was 16 months $(\mathrm{SD}=5)$ for true interval cancers, statistically significantly higher than 13 months $(\mathrm{SD}=5)$ for occult, 14 months $(\mathrm{SD}=9)$ for minimal signs and 13 months $(\mathrm{SD}=5)$ for missed (Table 1). The percentage of cancers diagnosed within the first 12 months after screening ranged from 24\% (83/353) for true interval cancers to $52 \%(66 / 246)$ for occult $(p<0.001)$, whereas the percentage of cancers diagnosed 19-24 months after screening ranged from $17 \%(21 / 126)$ for occult to $41 \%$ $(145 / 353)$ for true $(p<0.001)$ (Fig 4). 


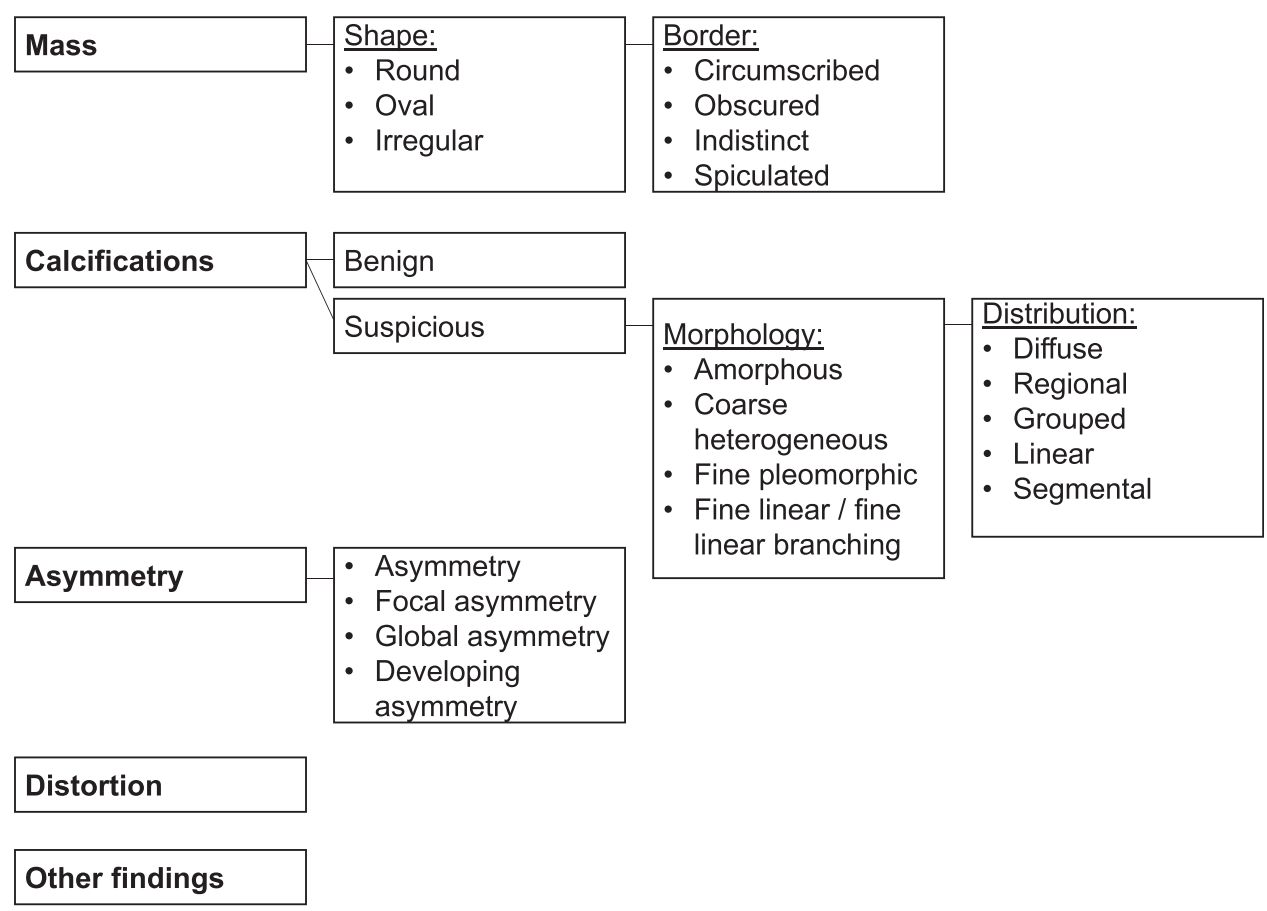

Figure 2. Classification of mammographic findings on diagnostic mammograms and prior screening mammograms, BI-RADS 5th edition.

\section{Mammographic Density}

Overall, 49\% (494/1010) of the cases were classified as having mammographic density a or b, and 51\% (516/1010) density c or $\mathrm{d}$. The distribution of mammographic density categories did not differ statistically between true, minimal signs and missed, ranging $50-53 \%$ for $\mathrm{a}+\mathrm{b}$ and $47-50 \%$ for $\mathrm{c}+\mathrm{d}$. However, among the occult cases, 31\% (38/126) were classified with mammographic density $\mathrm{a}+\mathrm{b}$ and $69 \%(88 / 126) \mathrm{c}+\mathrm{d}$, $p<0.001$ for occult compared with each of the other categories (Table 1$)$.

\section{Mammographic Findings}

The most frequent mammographic finding at diagnosis was a mass for all classification categories, ranging from 53\% (186/ $353)$ for true to $66 \%(163 / 246)$ for missed $(p<0.001)$ (Table 1). The second most frequent mammographic finding was asymmetry, ranging from $13 \%(31 / 246)$ for missed to $26 \%(90 / 353)$ for true $(p<0.001)$. The least frequent mammographic finding was calcifications for all classification categories, and no statistically significant differences were observed regarding the proportions of calcifications and distortions among cancers classified as true, minimal signs or missed. Median diameter of mammographic findings at diagnosis in the whole study population was $21 \mathrm{~mm}$ (IQR 1531), no statistically significant differences were observed between classification categories.

The most frequent mammographic finding on prior screening mammograms was asymmetry for both minimal signs $(65 \%, 186 / 285)$ and missed $(36 \%, 88 / 246 ; p<0.001)$ (Table 1). A mass was the least frequent finding among minimal signs $(5 \%, 15 / 285)$ and the second most frequent finding among missed $(32 \%, 79 / 246 ; p<0.001)$. Median diameter of mammographic findings at prior screening was $12 \mathrm{~mm}$ (IQR 8-17) for minimal signs and $15 \mathrm{~mm}$ (IQR 1123) for missed $(p<0.001)$.

Ninety-seven percent $(91 / 94)$ of masses at prior screening and $64 \%(176 / 274)$ of asymmetries at prior screening presented as masses at diagnosis. Further, the majority of calcifications at prior screening $(59 \%, 43 / 59)$ presented as calcifications also at diagnosis, whereas 60\% (54/90) of distortions remained distortions at diagnosis and 36\% (32/90) presented as a mass (Fig 5).

Among the masses missed at prior screening, 65\% (65/78) had irregular shape, and 90\% (70/78) had indistinct or spiculated border (Table 2). The most frequent minimal signs asymmetry was one-plane asymmetry (50\%, 94/186). The most frequent missed asymmetry was developing asymmetry $(51 \%, 45 / 88)$ which is defined as a focal (two-plane) asymmetry that is new, larger or more conspicuous than on the previous examination. At diagnosis, a round/oval mass was more frequent in true $(33 \%, 61 / 186)$ than missed $(17 \%, 27 /$ $163 ; p<0.001)$ interval cancer, otherwise no statistically significant differences were observed regarding subclasses of mammographic findings at diagnosis (Table 2).

\section{Histopathological Characteristics}

No differences in histopathological characteristics were observed between true, minimal sign or missed interval cancer. Ductal carcinoma in situ contributed with 5\% (49/1010) of all the cases, and the proportion did not differ between the 
(a)
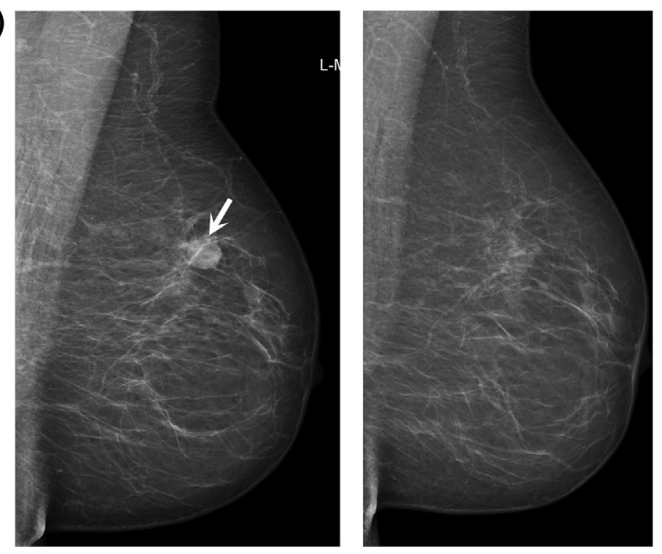

(c)
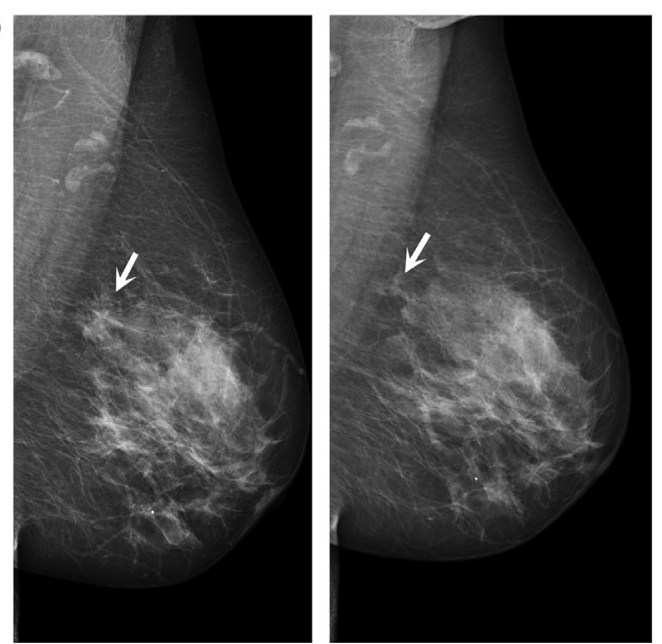

(b)

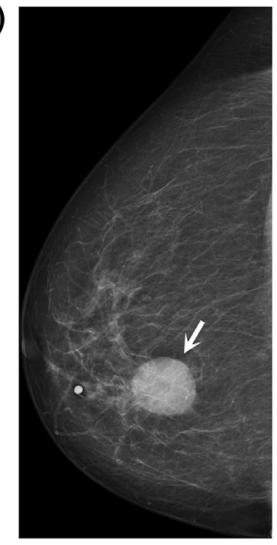

(d)

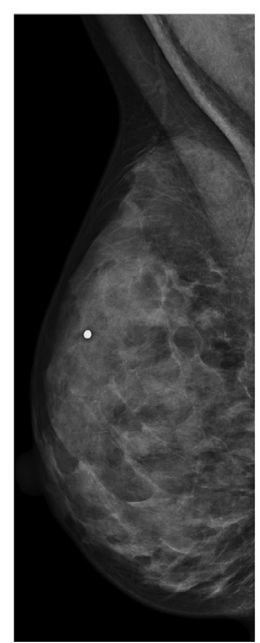

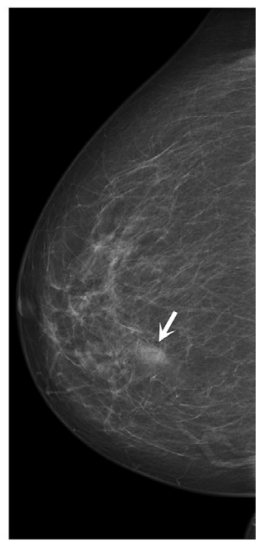

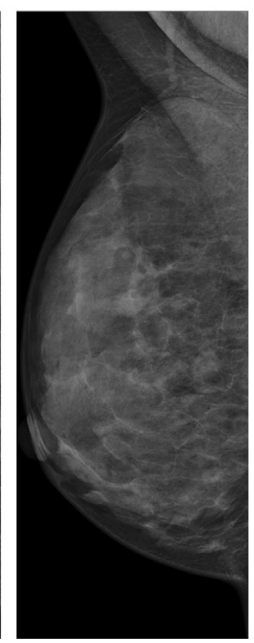

Figure 3. (a): True interval cancer: Left medio-lateral oblique (MLO) views of a 64-year-old woman presenting with a palpable lump and an indistinct round mass in the upper outer quadrant of the left breast (arrow) at diagnosis, diagnosed with a $16 \mathrm{~mm}$ invasive carcinoma of no special type (NST). No mammographic findings at prior screening. (b): Missed interval cancer: Right craniocaudal (CC) views of a 64-year-old woman presenting with a palpable lump and an obscured round mass in the medial aspect of the right breast (arrow) at diagnosis, diagnosed with a $25 \mathrm{~mm}$ invasive carcinoma of NST. A focal asymmetry (arrow) is visible at the cancer site on prior screening mammograms. (c): Minimal signs interval cancer: Left MLO views of a 69-year-old woman presenting with a palpable lump and an irregular spiculated mass in the upper outer quadrant of the left breast (arrow) at diagnosis, diagnosed with a $20 \mathrm{~mm}$ invasive carcinoma of NST. An asymmetry (arrow) is visible at the cancer site on prior screening mammograms. (d): Occult interval cancer: Right MLO views of a 53-year-old woman presenting with a palpable lump in the upper outer quadrant of the right breast and diagnosed with a multifocal invasive carcinoma. No visible mammographic findings at neither diagnosis nor prior screening.

classification categories (Table 3). The percentage of invasive carcinoma of NST ranged from $52 \%(66 / 126)$ for occult to $81 \%(284 / 353)$ for true interval cancer, $p<0.001$ for occult compared with true, minimal signs and missed. The percentage of invasive lobular carcinoma ranged from $11 \%$ for true $(38 / 353)$ and missed $(27 / 246)$ to $18 \%(22 / 126)$ for occult, $p=0.001$ for true versus occult. The highest proportion of "other invasive carcinomas", which included among others invasive tubular, medullar and mucinous carcinoma, was observed among occult cancers $(18 \%, 22 / 126)$ and the lowest among true $(5 \%, 17 / 353, p<0.001)$. No statistically significant differences in histopathological tumor diameter were observed between the review classification categories.

The percentage of Histological Grade 1 tumors was lowest for true interval cancers $(8 \%, 27 / 328)$ and highest for occult cancers $(25 \%, 26 / 105), p<0.001$. The smallest median histopathological tumor diameter was observed in occult cancers, $15 \mathrm{~mm}$ (IQR 10-23), $p$ $<0001$ compared with true, minimal signs and missed. No difference in median tumor diameter was observed between the other categories. The percentage of Histological Grade 3 tumors ranged from 30\% (32/105) for occult to $47 \%(153 / 328)$ for true interval cancer, $p=0.004$. There were no statistically significant differences in lymph node status between classification categories. Estrogen receptor positivity ranged from 73\% (243/ $332)$ for true to $91 \%(97 / 107)$ for occult cancers $(p<$ $0.001)$, whereas progesterone receptor positivity ranged from $51 \%(166 / 326)$ for true interval cancers to $65 \%$ $(68 / 105)$ for occult $(p=0.01$, Table 3$)$. 
TABLE 1. Age at Diagnosis (Mean and Standard Deviation, SD), Time Since Screening (Mean and SD), Distribution of Mammographic Density at Diagnosis, and Mammographic Findings on Diagnostic and Prior Screening Mammograms for 1010 Interval Breast Cancers, by Review Classification Categories (True, Occult, Minimal Signs and Missed).

\begin{tabular}{|c|c|c|c|c|c|}
\hline Variable & All & True & Occult & Minimal signs & Missed \\
\hline & $1010(100)$ & $353(35)$ & $126(13)$ & $285(28)$ & $246(24)$ \\
\hline Age, mean (SD) years & $61(6)$ & $61(6)$ & $60(6)$ & $61(6)$ & $62(5)$ \\
\hline Time since screening, mean (SD) months & $14(7)$ & $16(5)$ & $13(5)^{a}$ & $14(9)^{a}$ & $13(6)^{a}$ \\
\hline \multicolumn{6}{|l|}{ Mammographic density } \\
\hline BI-RADS a & $76(8)$ & $20(6)$ & $7(6)$ & $26(9)$ & $23(9)$ \\
\hline BI-RADS b & $418(41)$ & $160(45)^{b}$ & $31(25)$ & $118(41)$ & $109(44)^{\mathrm{b}}$ \\
\hline BI-RADS c & $420(42)$ & $142(40)$ & $56(44)$ & $122(43)$ & $100(41)$ \\
\hline BI-RADS d & $96(9)$ & $31(9)^{b}$ & $32(25)$ & $19(7)^{b}$ & $14(6)^{b}$ \\
\hline \multicolumn{6}{|l|}{ Diagnostic mammograms } \\
\hline Mass & $507(57)$ & $186(53)^{c}$ & . & $158(55)$ & $163(66)$ \\
\hline Calcifications & $64(7)$ & $20(6)$ & . & $20(7)$ & $24(10)$ \\
\hline Asymmetry & $179(20)$ & $90(26)^{c}$ & . & $58(20)$ & $31(13)$ \\
\hline Distortion & $130(15)$ & $53(15)$ & . & $49(17)$ & $28(11)$ \\
\hline Other findings & $4(1)$ & $4(1)$ & . & & \\
\hline Mammographic diameter, median (IQR) mm & $21(15,31)$ & $21(15,32)$ & . & $21(15,30)$ & $23(15,32)$ \\
\hline Data not available & 31 & 21 & . & 8 & 2 \\
\hline Prior screening mammograms & & & . & & \\
\hline Mass & $94(18)$ & . & . & $15(5)^{c}$ & $79(32)$ \\
\hline Calcifications & $73(14)$ & . & . & $34(12)$ & $39(16)$ \\
\hline Asymmetry & $274(52)$ & . & . & $186(65)^{c}$ & $88(36)$ \\
\hline Distortion & $90(17)$ & . & . & $50(18)$ & $40(16)$ \\
\hline Mammographic diameter, median (IQR) mm & $14(9,20)$ & . & . & $12(8,17)^{c}$ & $15(11,23)$ \\
\hline Data not available & 118 & . & . & 104 & 14 \\
\hline
\end{tabular}

BI-RADS, Breast Imaging-Reporting and Data System; IQR, inter-quartile range; SD, standard deviation.

Unless Otherwise Specified, Data are Number of Patients with Percentages in Parentheses.

a $p<0.001$ compared with true.

${ }^{\mathrm{b}} p<0.001$ compared with occult.

${ }^{\mathrm{c}} p<0.001$ compared with missed.

\section{Interval Versus Screen-Detected Cancer}

Comparative analyses of results from the present study and a parallel study of screen-detected breast cancer are presented in the Supplementary material (Supplementary Table 2). When combining true and occult cancers, no differences in the distribution of true/occult, minimal sign and missed cancers were observed. A higher percentage of BI-RADS $\mathrm{c}$ and

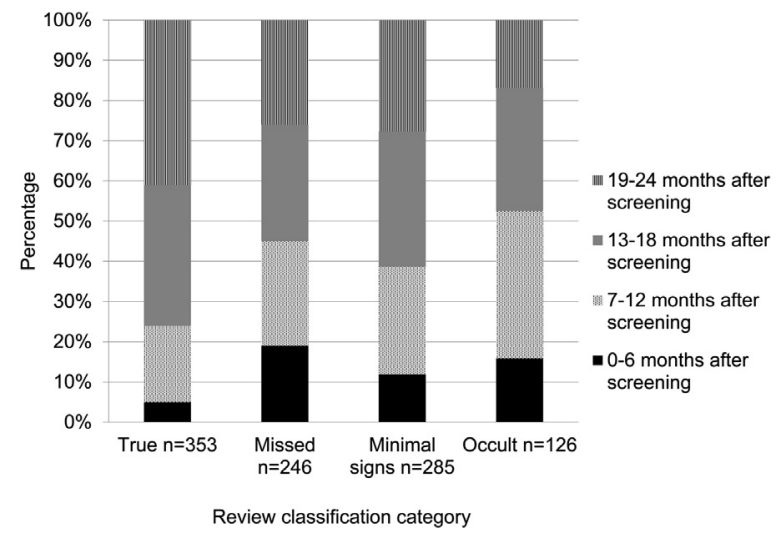

Figure 4. Time since screening by review category. d mammographic density was observed in interval cancers (51\%, 516/1010) compared with screen-detected (34\%, 417/ 1257), $p<0.001$. The proportion of calcifications were higher in screen-detected than interval cancer, both at diagnosis and prior screening $(p<0.001)$, and the proportion of distortions was higher in interval cancer $(p<0.001)$. Further, a statistically significantly lower proportion of DCIS, a larger

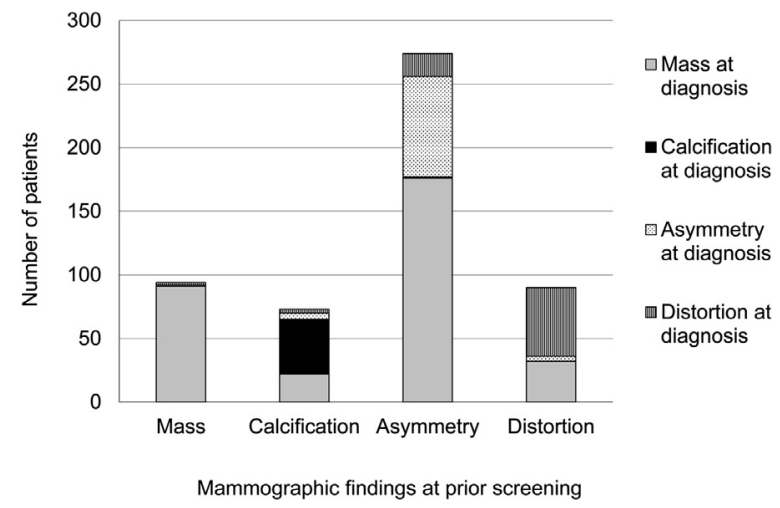

Figure 5. Number of patients with mammographic findings at diagnosis by findings at prior screening. 
TABLE 2. Subclassification of Mammographic Findings on Prior Screening and Diagnostic Mammograms.

$(n=285)$

\begin{tabular}{|c|c|c|c|c|c|}
\hline & \multicolumn{2}{|c|}{ Prior screening Mammograms } & \multicolumn{3}{|c|}{ Diagnostic Mammograms } \\
\hline & $\begin{array}{l}\text { Minimal Signs } \\
\quad(n=285)\end{array}$ & Missed $(n=246)$ & True $(n=353)$ & $\begin{array}{l}\text { Minimal Signs } \\
\quad(n=285)\end{array}$ & Missed $(n=246)$ \\
\hline \multicolumn{6}{|l|}{ Mass } \\
\hline \multicolumn{6}{|l|}{ Shape } \\
\hline Round/oval & $11(92)^{a}$ & $27(35)$ & $61(33)^{a}$ & $36(23)$ & $27(17)$ \\
\hline Irregular & $1(8)$ & $51(65)$ & $125(67)$ & $122(77)$ & $136(83)$ \\
\hline Data not available & 3 & 1 & & & \\
\hline \multicolumn{6}{|l|}{ Border } \\
\hline Circumscribed/obscured/microlobulated & $6(55)$ & $8(10)$ & $22(12)$ & $12(8)$ & $14(9)$ \\
\hline Indistinct/spiculated & $5(45)$ & $70(90)$ & $164(88)$ & $146(92)$ & $149(91)$ \\
\hline Data not available & 4 & 1 & & & \\
\hline \multicolumn{6}{|l|}{ Calcifications } \\
\hline Typically benign & $8(24)$ & $1(3)$ & . & . & . \\
\hline Suspicious & $26(76)$ & $38(97)$ & . & . & . \\
\hline \multicolumn{6}{|l|}{ Morhology suspicious calcfications } \\
\hline Amorphous/Coarse heterogenous & $10(38)$ & $5(14)$ & $3(15)$ & . & $3(13)$ \\
\hline Fine plemorphic/linear/branching & $16(62)$ & $32(86)$ & $17(85)$ & $20(100)$ & $21(87)$ \\
\hline Data not available & & 1 & & & \\
\hline \multicolumn{6}{|l|}{ Distribution suspicious calcifications } \\
\hline Diffuse/regional & $6(23)$ & $14(37)$ & $6(30)$ & $13(65)$ & $8(33)$ \\
\hline Grouped & $16(62)$ & $15(39)$ & $9(45)$ & $5(25)$ & $10(42)$ \\
\hline Linear/segmental & $4(15)$ & $9(24)$ & $5(25)$ & $2(10)$ & $6(25)$ \\
\hline \multicolumn{6}{|l|}{ Asymmetry } \\
\hline Asymmetry & $94(50)^{a}$ & $19(22)$ & $24(27)$ & $17(29)$ & $5(16)$ \\
\hline Focal/global asymmetry & $35(19)$ & $24(27)$ & $17(19)$ & $16(28)$ & $15(48)$ \\
\hline Developing asymmetry & $57(31)$ & $45(51)$ & $49(54)$ & $25(43)$ & $11(36)$ \\
\hline
\end{tabular}

Data are Number of Patients with Percentages in Parentheses.

${ }^{a} p<0.001$ compared with missed.

TABLE 3. Histopathological Characteristics by Review Classification Categories.

\begin{tabular}{|c|c|c|c|c|c|}
\hline & Total & True & Occult & Minimal Signs & Missed \\
\hline \multicolumn{6}{|l|}{ Histopathological tumor type } \\
\hline Ductal carcinoma in situ & $49(5)$ & $14(4)$ & $11(9)$ & $13(5)$ & $11(5)$ \\
\hline Invasive carcinoma of NST & $754(75)$ & $284(81)^{a}$ & $66(52)$ & $214(75)^{a}$ & $190(77)^{a}$ \\
\hline Invasive lobular carcinoma & $129(13)$ & $38(11)$ & $28(22)$ & $36(13)$ & $27(11)$ \\
\hline Other invasive carcinoma & $78(8)$ & $17(5)^{a}$ & $21(17)$ & $22(8)$ & $18(7)$ \\
\hline Tumor diameter, median (IQR) mm & $19(13,25)$ & $19(14,26)^{a}$ & $15(10,23)$ & $20(14,25)^{a}$ & $20(13,26)^{a}$ \\
\hline Data not available & 75 & 28 & 16 & 19 & 12 \\
\hline \multicolumn{6}{|l|}{ Histological Grade } \\
\hline Grade 1 & $128(14)$ & $27(8)^{a}$ & $26(25)$ & $44(16)$ & $31(14)$ \\
\hline Grade 2 & $437(47)$ & $148(45)$ & $47(45)$ & $126(47)$ & $116(51)$ \\
\hline Grade 3 & $364(39)$ & $153(47)$ & $32(30)$ & $98(37)$ & $81(36)$ \\
\hline Data not available & 32 & 11 & 10 & 4 & 7 \\
\hline \multicolumn{6}{|l|}{ Lymph node status } \\
\hline Positive & $384(41)$ & $149(46)$ & $33(31)$ & $105(40)$ & $97(42)$ \\
\hline Data not available & 34 & 12 & 8 & 8 & 6 \\
\hline \multicolumn{6}{|l|}{ Hormonal receptor status } \\
\hline Estrogen receptor positive & $745(79)$ & $243(73)^{a}$ & $97(91)$ & $217(81)$ & $188(81)$ \\
\hline Data not available & 21 & 6 & 8 & 4 & 3 \\
\hline Progesterone receptor positive & $542(58)$ & $166(51)$ & $68(65)$ & $166(62)$ & $142(62)$ \\
\hline Data not available & 33 & 12 & 10 & 6 & 5 \\
\hline
\end{tabular}

IQR, interquartile range; NST, no special type.

Tumor diameter, Histological Grade, lymph node status and hormonal receptor status for invasive cancers only. Unless otherwise is specified, data are number of patients and percentages.

${ }^{a} p<0.001$ compared with occult. 
tumor diameter, and higher proportions of Histological Grade 3 invasive cancer, lymph node positive and hormone receptor negative tumors were observed in interval cancer compared with screen-detected.

\section{DISCUSSION}

In this fully informed, consensus-based, retrospective review of diagnostic and prior screening mammograms of 1010 interval breast cancer cases in BreastScreen Norway, the radiologists classified 35\% of the cases as true interval cancer, $13 \%$ as occult, $28 \%$ as minimal signs and $24 \%$ as missed. Mean time between screening and diagnosis was shortest for occult and longest for true interval cancer. Sixty-nine percent of the mammograms from women with occult cancer were classified as BI-RADS density c or d, higher than all other classification categories. Among cancers classified as missed, about $1 / 3$ were masses and $1 / 3$ asymmetries at prior screening. Ninety percent of the missed masses had indistinct or spiculated borders and half of the missed asymmetries were developing asymmetries. We observed a direction of the findings for true interval cancers towards less favorable histopathological characteristics compared with the other classification categories. However, only Histological Grade 1 and estrogen receptor negative tumors differed statistically; lower in true versus occult interval cancers for both. A smaller median tumor diameter was observed in occult cancers compared with all other categories.

\section{Proportions of True and Missed Interval Cancer}

Our results on classification of interval cancer based on findings on prior screening mammograms are in line with other studies. In four different review studies, all with an informed study design, the percentage of true/occult interval cancer ranged from 38\%-59\%, and missed interval cancer ranged from $22 \%-35 \%(15,21-23)$. In a blinded review of interval cancer, $55 \%$ were classified as true/occult and $21 \%$ as missed (9). The review logistics differ between studies, from blinded to fully informed, and from individual to consensus-based. Little consensus exists on standardization of study design in reviews of interval cancer, thus, comparison between studies is challenging (4). Further, the definition of interval cancer as such as well as the subdivision of the radiological classification groups vary (2). In general, the more information available to reviewers, the higher the proportion of missed cancer cases. This might be due to bias in the review process as the reviewers are aware of where the cancer is located and thus, where to look for even subtle findings on prior mammograms. Further, the closer the review is to a normal screening situation, the lower the proportion classified as missed cancer, associated with the readers' level of expectation to detect interval cancers (24-27). Our fully informed consensus-based study design is thus the design that yields the highest proportion of missed cancer at review.

\section{Mammographic Findings on Prior Screening Mammograms - Missed and Minimal Signs}

Irregular, spiculated masses are considered more suspicious for cancer than round/oval and circumscribed masses; developing asymmetries are in general more suspicious than one-plane asymmetries, and fine pleomorphic or linear/branching calcifications more indicative of malignancy than amorphous (20). Obviously, in a retrospective review, the allocation of exams into the categories minimal signs or missed is based on the level of suspiciousness of the mammographic findings. The vast majority of missed masses at screening in our study were irregular in shape, with indistinct or spiculated border. Such findings are suspicious of malignancy and usually result in a recall if perceived. Thus, it is likely that a major reason for failure to recall these cases was misperception or reduced vigilance among the screen-readers. However, even suspicious findings are sometimes refrained from recall, especially if stable or slowgrowing through consecutive screening rounds (28). Nevertheless, these findings may benefit from recall, even if they are probably not representing the most aggressive cancers. As we only classified one prior screening exam, we have no data on long-term stability of mammographic findings in our study. Almost all masses at prior screening remained masses at diagnosis, and the majority of asymmetries at prior screening turned into masses at diagnosis. This may illustrate a pathway from asymmetry to mass, and is in accordance with our findings for screen-detected breast cancer (19). Further, as for missed screen-detected cancer, the majority of calcifications and distortions remained the same at diagnosis.

We defined minimal signs as minor or non-specific findings in which recall was not considered mandatory or even not possible within the screening program. Hence, the mammographic findings of these cancers are less suspicious than in the missed cancers; frequent findings in prior screening mammograms were one-plane asymmetries and round/oval circumscribed or obscured masses. Such findings are common in screening mammography and usually represent benign or normal findings. Strategies to improve the radiologists reading skills, including sensitivity and specificity, may change the threshold for which findings that require further assessment to be ruled out. This might be related to the usual and accepted recall rate of the actual screening program, which vary across different countries. In programs with generally low recall rate, the acceptance for not recalling subtle or minor findings would probably be higher than in programs with a higher recall rate. Further, including more sets of prior screening mammograms at screening reading may be a proper strategy to improve sensitivity and specificity as the radiologists may become more aware of subtle changes $(19,29)$.

\section{Mammographic Density and Interval Cancer}

In general, the sensitivity of mammography in detecting breast cancer is lower in dense compared with fatty breasts (30). In line with this, and also in keeping with other studies, we 
observed a statistically higher mammographic density in women with occult interval cancer compared with all other classification groups $(15,31)$, and also for interval versus screendetected cancer. Implementation of supplemental imaging techniques has been proposed as part of stratified screening based on differences in breast cancer risk and mammographic density (32). In studies, digital breast tomosynthesis (DBT) has increased the rate of screen-detected cancer (32-37), probably by increasing the conspicuity of lesions and reducing the masking effect of breast tissue. However, an increase has not been observed in the highest density category (32). Further, studies have not shown a reduction in interval cancer rates for screening with DBT, and whether DBT may have the potential to reduce interval cancer in dense breasts is not yet demonstrated. Hand-held or automatic breast ultrasound (ABUS) or contrast-enhanced breast MRI is also associated with increased sensitivity for breast cancer in dense breasts (38-40). These techniques may also reduce the occurrence of interval breast cancer, including occult breast cancer outside the anatomical target area for mammography. However, implementation of stratified screening with more resource intensive imaging techniques needs a thorough evaluation of benefits, risks and cost-effectiveness. Further, in our study, only $9 \%$ of the interval cancers were actually seen in breasts with BI-RADS d mammographic density.

\section{Screening Interval}

Two thirds of the interval cancers were diagnosed in the second half of the screening interval, which is in keeping with other studies $(9,23)$. A shortening of the screening interval could thus reduce the number of interval cancers. This could particularly have an effect on the occurrence of true interval cancer, as this group has the highest frequency of cancers diagnosed $>12$ months after screening. BreastScreen Norway and most other European breast cancer screening programs, screen women biennially, whereas in the United Kingdom (UK) screening is triennial, and in the United States (US) usually annual. In UK the interval cancer rate $<24$ months after screening is comparable with the rates in BreastScreen Norway, but because of additional interval cancers during the third year of the screening interval the total interval cancer rate is higher (41). Studies comparing interval cancer rates have demonstrated higher rates of interval cancer in the US compared with Norway, despite annual screening in the US $(42,43)$. This is possibly due to additional differences in screening regimes between the US and European countries, among other single-reading, different screening logistics, lower reading volume and early recalls. Even if reducing the screening interval to 1 year has the potential to reduce the interval cancer rate, this could decrease the cost-effectiveness of the screening program and potentially increase possible risks as false positives. The European Commission Initiative on Breast Cancer (ECIBC) have agreed on a strong recommendation against annual screening (44). This is in contrast to the American College of Radiology and the Society of Breast
Imaging, which recommend annual mammography, while the American Cancer Society and the American Society of Surgical Oncologists recommend annual mammography to age 55, then biennial. The United States Preventive Services Task Force recommends biennial screening (45).

\section{Histopathological Findings}

Histopathological characteristics of tumors classified as true and missed vary between studies (2). In some studies missed interval cancers have a larger tumor diameter and are more often lymph node positive $(9,21,23)$ though more frequently low-graded than true interval cancers, whereas others observe less favorable histopathological characteristics among true, in particular higher proportions of high Histologic Grade and hormone receptor negative disease $(15,31,46)$. We did not find any histopathological differences between true and missed interval cancer, whereas true interval cancer more frequently was of Histologic Grade 3 and hormone receptor negative than occult. Some retrospective reviews combine true and occult cancers into one category, based on the lack of mammographic findings at prior screening. However, due to the differences observed in histopathological characteristics as well as in the time interval between screening and diagnosis, it seems reasonable to separate true and occult interval cancers as two different categories at review.

\section{Interval Versus Screen-Detected Breast Cancer}

When combining true and occult cancer into one category, as none of these show detectable findings on prior screening mammograms, no differences in the distribution of categories were observed between interval and screen-detected cancer (19). The distribution of mammographic density, however, differed statistically significantly between interval and screen-detected cancer, and the difference also holds when excluding the occult cancers. These finding is in line with other studies $(15,30)$. In keeping with the literature, less favorable tumor characteristics were observed in interval cancers compared with screen-detected, the proportion of DCIS was lower in interval versus screendetected cancers whereas the proportion of ILC was higher $(47,48)$. Calcifications were less frequent in interval than screen-detected cancers, both at diagnosis and prior screening, which is probably related to the lower proportion of DCIS. Further, a higher proportion of distortions was observed at diagnosis in interval cancers, which might reflect the higher proportion of ILC. Interestingly, except for calcifications, no differences in the distribution of other mammographic findings were observed at prior screening, indicating that the radiologists may overlook or refrain to recall the same type of lesions in interval and screen-detected cancer. 


\section{Possible Strategies to Reduce Interval Cancer and Missed Cancer at Screening}

As described previously, a shortening of the screening interval as well as implementation of supplementary screening techniques may decrease the rates of interval cancer, although such measures may not unanimously increase the overall quality of the screening program. Education, including self-assessment and training schemes, and participation in reviews could be ways to improve the screen-reader's sensitivity to more subtle findings. These strategies may also improve the radiologists' perception and interpretation, and increase their awareness of possible pitfalls. The experience in mammography screening is also important for the radiologist's sensitivity. A Norwegian study demonstrated optimal performance in screen-reading with an annual reading volume of $4000-10,000$ mammograms and a cumulative volume of at least 20,000 mammograms. However, sensitivity decreased for annual volumes $>10,000$ mammograms and cumulative volumes $>100,000$, which may indicate that too high workloads could cause fatigue and reduced attention among the screen-readers (18). Double reading is shown to increase cancer detection (49), and probably also reduce the occurrence of interval cancer; in a study from BreastScreen Norway, 24\% of the screendetected cancers were detected by only one reader (50). Optimization of image quality is also of outmost importance to reduce all types of interval breast cancer. In addition to the technical image parameters, optimal positioning is crucial; some interval cancers may be classified as occult due to suboptimal positioning leaving the tumor incorrectly outside the field of view. Finally, during the past years, artificial intelligence (AI) based on deep convolutional neural networks shows promising results in breast diagnostics and screening (51-53) and if AI in the future demonstrates the ability to detect abnormalities in images not perceived by radiologists, or not even detectable by the human eye, it may be possible to lower the interval cancer rate.

\section{Strength and Limitations}

The large study sample represents strength in our study. Further, all images were digital, and the study is therefore relevant for the standard screening technique today, in contrast to most prior review studies using screen-film mammography. However, as only DM screening mammograms were included, the external generalizability to screening with DBT was limited. The fully informed consensus-based review design is the design resulting in the highest proportion cancers classified as missed, and least resembling a normal screening situation. This may limit the external generalizability of the results. We chose this design for practical and educational purposes. Further, the study included radiologists from all breast centers and except one (T.H.), all reviewers participated in only two of the 16 reviews. Different pathologists at all centers performed histopathological analyses, and differences between centers may have occurred. Finally, histopathological tumor diameter was missing in 77 cases. This is probably due to neo-adjuvant treatment of the cancers, often resulting in a non-measurable tumor burden at surgery, and illustrated by a higher median mammographic diameter for cases with missing tumor diameter compared with no missing tumor diameter (35 versus $21 \mathrm{~mm}$ ).

\section{CONCLUSION}

In an informed, retrospective review of interval cancers, $1 / 4$ of the cancers were classified as missed at screening, with a potential of earlier diagnosis. Even if retrospective reviews do not reflect a normal screening setting and hindsight findings are not always signs of screening failure, we consider reviews important in the continuous work to assure and improve the quality of the radiologists and the screening programs. Both organized high volume review studies as well as individual reviews of cancer cases personally screened by the screenreader are valuable in that respect.

\section{ACKNOWLEDGEMENTS}

Vestre Viken Hospital Trust funded the salary for TH during the project period. $\mathrm{SH}$ has permanent employment as a researcher at the Cancer Registry of Norway, independent of her job as administrative leader of BreastScreen Norway. We want to thank all the radiologists who participated in the reviews: Gunn Aagedal Hervold and Helene Sandvik Solli, Sørlandet Hospital Kristiansand; Manouchehr Seyedzadeh and Maryam Lahooti, Akershus University Hospital; AnnaLena Skoglund and Lars Rostgard Christensen, Innlandet Hospital Hamar; Tone Neraas, Hildegunn Aase, Kristian Søvik and Kerstin Obst-Gleditsch, Haukeland University Hospital; Fride Tennebø, Unni Bergan and Arjan Christiansen, Møre og Romsdal Hospital Trust Ålesund; Enno Rodegerdts and Heinrich August Backmann, Nordland Hospital Trust; Marit Muri Holmen and Helene Bertelsen Brandal, Oslo University Hospital; Lars Hagen Henriksen and Hanne Rosenquist, Innlandet Hospital Lillehammer; Bodil Styr and Nataliya Buset, Stavanger University Hospital; Bernadett Hrabovsky and Zsolt Francziszty, Førde Hospital Trust; Marit Solveig Bekken and Anne-Lina Romsas Fjellhøy, Telemark Hospital Trust; Rica Mortensen and Berit Steene, The University Hospital of North Norway; Agnes Ostlie and Trine Holmen, St. Olav's Hospital, Trondheim University Hospital; Judy Albertsen and Marte Myrebøe Schultze, Vestfold Hospital Trust; Hilde Bjørndal and Annette Bergene Holm, Vestre Viken Hospital Trust; Merete Kristiansen, Magne Kolstad Skarheim and Hege Nordlie, Østfold Hospital Trust. We also want to thank Anne Kathrin Olsen Ertzaas, Asne Holen and Wenche Melby from the Cancer Registry of Norway.

\section{REFERENCES}

1. Perry $N$, Broeders $M$, de Wolf $C$, et al. European guidelines for quality assurance in breast cancer screening and diagnosis. Brussels, Belgium: European Communities, 2006. 
2. Houssami N, Hunter K. The epidemiology, radiology and biological characteristics of interval breast cancers in population mammography screening. NPJ Breast Cancer 2017; 3:12.

3. Messinger J, Crawford S, Roland L, et al. Review of subtypes of interva breast cancers with discussion of radiographic findings. Curr Probl Diagn Radiol 2019; 48(6):592-598.

4. Houssami N, Irwig L, Ciatto S. Radiological surveillance of interval breast cancers in screening programmes. Lancet Oncol 2006; 7(3):259-265.

5. Bellio G, Marion R, Giudici F, et al. Interval breast cancer versus screendetected cancer-comparison of clinicopathologic characteristics in a single-center analysis. Clin Breast Cancer 2017; 17(7):564-571.

6. Cheasley D, Li N, Rowley SM, et al. Molecular comparison of interval and screen-detected breast cancers. J Pathol 2019; 248(2):243-252.

7. Gilliland FD, Joste N, Stauber PM, et al. Biologic characteristics of interval and screen-detected breast cancers. J Natl Cancer Inst 2000; 92 (9):743-749.

8. Wishart GC, Greenberg DC, Britton PD, et al. Screen-detected vs symptomatic breast cancer-is improved survival due to stage migration alone? Br J Cancer 2008; 98(11):1741-1744.

9. Carbonaro LA, Azzarone A, Paskeh BB, et al. Interval breast cancersabsolute and proportional incidence and blinded review in a community mammographic screening program. Eur J Radiol 2014; 83(2):e84-e91.

10. Tornberg S, Kemetli L, Ascunce N, et al. A pooled analysis of interval cancer rates in six European countries. Eur J Cancer Prev 2010; 19 (2):87-93.

11. Andersen SB, Tornberg S, Lynge E, et al. A simple way to measure the burden of interval cancers in breast cancer screening. BMC Cancer 2014; $14: 782$

12. Bulliard JL, Sasieni $P$, Klabunde C, et al. Methodological issues in inter national comparison of interval breast cancers. Int J Cancer 2006; 119 (5):1158-1163.

13. Hofvind S, Tsuruda K, Mangerud G, Ertzaas A, Holen A, Pedersen K, et al. The Norwegian Breast Cancer Screening Program, 1996-2016: Celebrating 20 years of organised mammographic screening. Cancer in Norway 2016 - Cancer incidence, mortality, survival and prevalence in Norway: Cancer Registry of Norway; 2017

14. Boyd NF, Huszti E, Melnichouk O, et al. Mammographic features associated with interval breast cancers in screening programs. Breast Cancer Res 2014; 16(4):417

15. Domingo L, Salas D, Zubizarreta $R$, et al. Tumor phenotype and breast density in distinct categories of interval cancer: results of populationbased mammography screening in Spain. Breast Cancer Res 2014; 16 (1):R3.

16. Maclnnes EG, Duffy SW, Simpson JA, et al. Radiological audit of interval breast cancers: Estimation of tumor growth rates. Breast 2020; 51:114-119.

17. Ministry of Health and Care Services, Forskrift om innsamling og behandling av helseopplysninger i Kreftregisteret (The Cancer Registry Regulation), (2001).

18. Hoff SR, Myklebust TA, Lee Cl, Hofvind S. Influence of Mammography Volume on Radiologists' Performance: Results from BreastScreen Norway. Radiology. 2019;292(2):289-96.

19. Hovda T, Tsuruda K, Hoff SR, Sahlberg KK, Hofvind S. Radiological review of prior screening mammograms of screen-detected breast cancer. Eur Radiol. 2021;31(4):2568-79

20. Sickles E, D'Orsi CJ, Bassett LW, et al. ACR BI-RADS Mammography ACR BI-RADS Atlas, Breast Imaging Reporting and Data System. Reston, VA: American College of Radiology, 2013.

21. Hofvind S, Geller B, Skaane P. Mammographic features and histopathological findings of interval breast cancers. Acta radiologica (Stockholm, Sweden: 1987) 2008; 49(9):975-981

22. Hoff SR, Samset JH, Abrahamsen AL, et al. Missed and true interval and screen-detected breast cancers in a population based screening program. Acad Radiol 2011; 18(4):454-460.

23. Weber RJ, van Bommel RM, Louwman MW, et al. Characteristics and prog nosis of interval cancers after biennial screen-film or full-field digital screening mammography. Breast Cancer Res Treat 2016; 158(3):471-483.

24. Gordon PB, Borugian MJ, Warren Burhenne LJ. A true screening environment for review of interval breast cancers - pilot study to reduce bias. Radiology 2007; 245(2):411-415

25. Hofvind S, Skaane P, Vitak B, et al. Influence of review design on percentages of missed interval breast cancers: retrospective study of interval cancers in a population-based screening program. Radiology 2005; 237 (2):437-443.
26. Ciatto S, Catarzi S, Lamberini MP, et al. Interval breast cancers in screening: the effect of mammography review method on classification. Breast 2007; 16(6):646-652.

27. Moberg K, Grundstrom H, Tornberg S, et al. Two models for radiological reviewing of interval cancers. J Med Screen 1999; 6(1):35-39.

28. Lamb LR, Mohallem Fonseca M, Verma R, et al. Missed breast cancereffects of subconscious bias and lesion characteristics. Radiographics 2020; 40(4):941-960.

29. Roberts-Klein S, luanow E, Slanetz PJ. Avoiding pitfalls in mammographic interpretation. Can Assoc Radiol J 2011; 62(1):50-59.

30. Boyd NF, Guo H, Martin LJ, et al. Mammographic density and the risk and detection of breast cancer. N Engl J Med 2007; 356(3):227-236.

31. Evans AJ, Kutt E, Record C, et al. Radiological and pathological findings of interval cancers in a multi-centre, randomized, controlled trial of mammographic screening in women from age 40-41 years. Clin Radiol 2007; 62(4):348-352.

32. Moshina N. Comparing screening outcomes for digital breast tomosynthesis and digital mammography by automated breast density in a randomized controlled trial: Results from the To-Be trial Radiology. 2020.

33. Skaane P, Bandos Al, Niklason LT, et al. Digital mammography versus digital mammography plus tomosynthesis in breast cancer screeningthe oslo tomosynthesis screening trial. Radiology 2019; 291(1):23-30.

34. Hofvind S, Hovda T, Holen AS, et al. Digital breast tomosynthesis and synthetic $2 \mathrm{~d}$ mammography versus digital mammography-evaluation in a population-based screening program. Radiology 2018; 287(3): 787-794.

35. Zackrisson S, Lang K, Rosso A, et al. One-view breast tomosynthesis versus two-view mammography in the Malmo Breast Tomosynthesis Screening Trial (MBTST): a prospective, population-based, diagnostic accuracy study. Lancet Oncol 2018; 19(11):1493-1503.

36. Bernardi D, Macaskill P, Pellegrini M, et al. Breast cancer screening with tomosynthesis (3D mammography) with acquired or synthetic 2D mammography compared with 2D mammography alone (STORM-2) - a population-based prospective study. Lancet Oncol 2016; 17(8):1105-1113.

37. Marinovich ML, Hunter KE, Macaskill $P$, et al. Breast cancer screening using tomosynthesis or mammography: a meta-analysis of cancer detection and recall. J Natl Cancer Inst 2018; 110(9):942-949.

38. Comstock CE, Gatsonis C, Newstead GM, et al. Comparison of abbreviated breast MRI vs digital breast tomosynthesis for breast cancer detection among women with dense breasts undergoing screening. JAMA 2020; 323(8):746-756

39. Rella R, Belli P, Giuliani M, et al. Automated breast ultrasonography (abus) in the screening and diagnostic setting-indications and practical use. Acad Radiol 2018; 25(11):1457-1470.

40. Bakker MF, de Lange SV, Pijnappel RM, et al. Supplemental MRI screening for women with extremely dense breast tissue. N Engl J Med 2019; 381(22):2091-2102.

41. National Health Service Breast Screening Programme. National collation of breast interval cancer data. NHSBSP Occational Report 12/03. 2012.

42. Hofvind S, Vacek PM, Skelly J, Weaver DL, Geller BM. Comparing screening mammography for early breast cancer detection in Vermont and Norway. J Natl Cancer Inst. 2008;100(15):1082-91.

43. Hofvind S, Yankaskas BC, Bulliard JL, Klabunde CN, Fracheboud J. Comparing interval breast cancer rates in Norway and North Carolina: results and challenges. J Med Screen. 2009;16(3):131-9.

44. ECIBC. Recommendations from the European Breast Cancer Guidelines [updated 28/05/2020. Available from: https://healthcare-quality.jrc.ec. europa.eu/european-breast-cancer-guidelines. Accessed June 2020.

45. Arleo EK, Hendrick RE, Helvie MA, et al. Comparison of recommendations for screening mammography using CISNET models. Cancer 2017; 123(19):3673-3680.

46. Porter GJ, Evans AJ, Burrell HC, et al. Interval breast cancers: prognostic features and survival by subtype and time since screening. J Med Screen 2006; 13(3):115-122.

47. Meshkat B, Prichard RS, Al-Hilli Z, et al. A comparison of clinical-pathological characteristics between symptomatic and interval breast cancer. Breast 2015; 24(3):278-282.

48. Bare M, Tora N, Salas D, et al. Mammographic and clinical characteristics of different phenotypes of screen-detected and interval breast cancers in a nationwide screening program. Breast Cancer Res Treat 2015; 154(2):403-415

49. Brennan PC, Ganesan A, Eckstein MP, et al. Benefits of independent double reading in digital mammography - a theoretical evaluation of all possible pairing methodologies. Acad Radiol 2019; 26(6):717-723. 
50. Hofvind S, Geller BM, Rosenberg RD, et al. Screening-detected breast cancers: discordant independent double reading in a population-based screening program. Radiology 2009; 253(3):652-660.

51. Rodriguez-Ruiz A, Krupinski E, Mordang JJ, et al. Detection of breast cancer with mammography-effect of an artificial intelligence support system. Radiology 2019; 290(2):305-314.
52. Lang K, Hofvind S, Rodriguez Ruiz A, et al. Can artificial intelligence reduce the interval cancer rate in mammography screening? Eur Radiol. 2020. doi:10.1007/s00330-021-07686-3. Online ahead of print.

53. Sechopoulos I, Teuwen J, Mann R. Artificial intelligence for breast cancer detection in mammography and digital breast tomosynthesis - state of the art. Semin Cancer Biol 2020. doi:10.1016/j.semcancer.2020.06.002. Online ahead of print. 